\title{
Environmental Quality and the U.S. Power Sector: Air Quality, Water Quality, Land Use and Environmental Justice
}

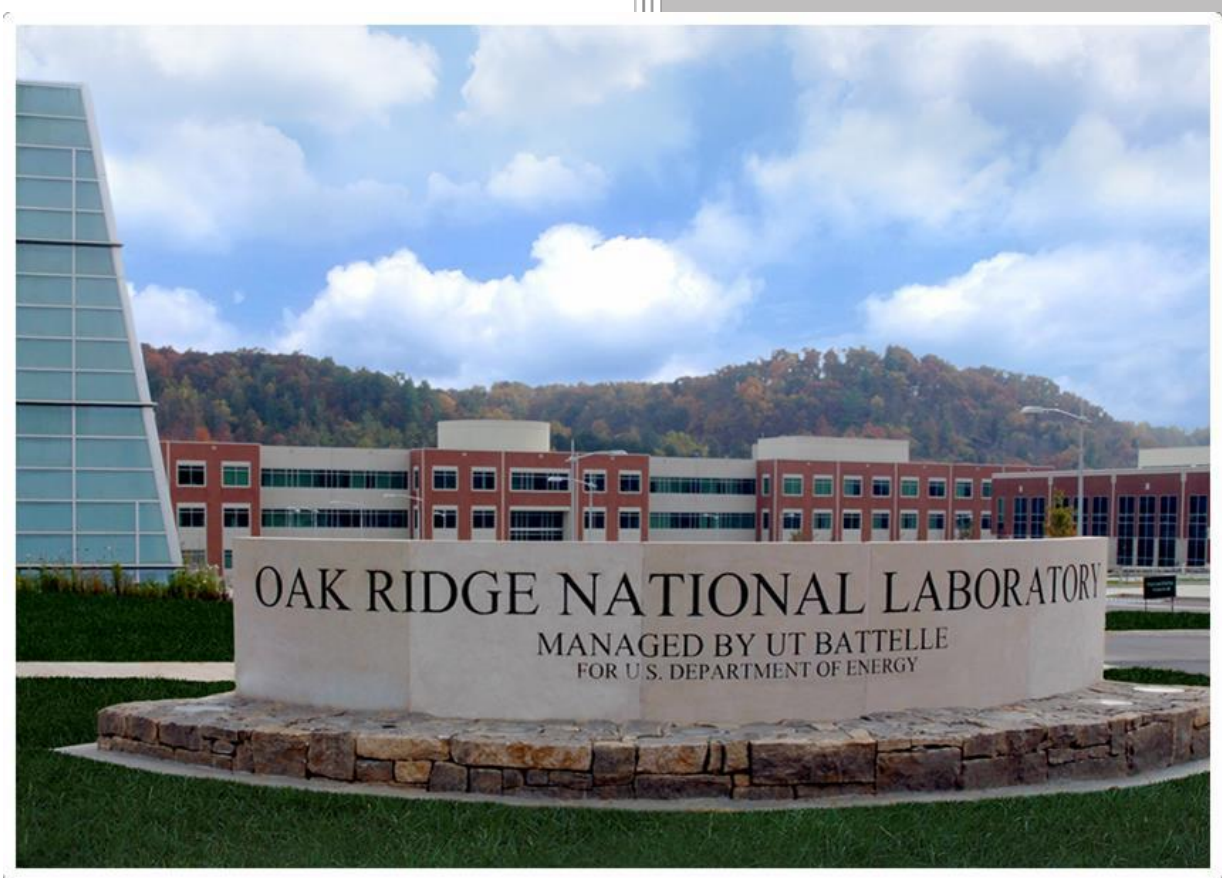

Approved for public release. Distribution is unlimited.
Emanuele Massetti Marilyn A. Brown Melissa Lapsa Isha Sharma James Bradbury Colin Cunliff Yufei Li

January 4, 2017 


\section{DOCUMENT AVAILABILITY}

Reports produced after January 1, 1996, are generally available free via US Department of Energy (DOE) SciTech Connect.

Website http://www.osti.gov/scitech/

Reports produced before January 1,1996, may be purchased by members of the public from the following source:

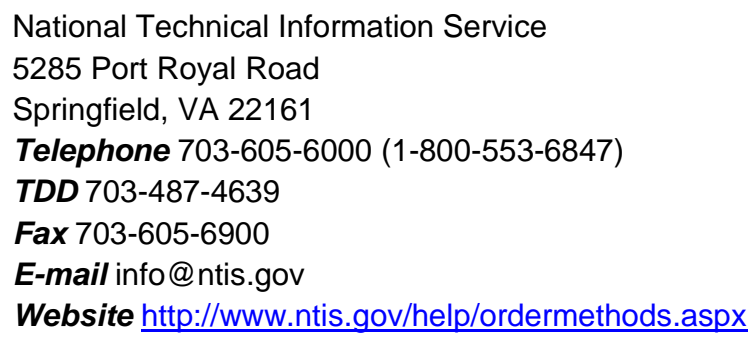

Reports are available to DOE employees, DOE contractors, Energy Technology Data Exchange representatives, and International Nuclear Information System representatives from the following source:

Office of Scientific and Technical Information

PO Box 62

Oak Ridge, TN 37831

Telephone 865-576-8401

Fax 865-576-5728

E-mail reports@osti.gov

Website http://www.osti.gov/contact.html

This report was prepared as an account of work sponsored by an agency of the United States Government. Neither the United States Government nor any agency thereof, nor any of their employees, makes any warranty, express or implied, or assumes any legal liability or responsibility for the accuracy, completeness, or usefulness of any information, apparatus, product, or process disclosed, or represents that its use would not infringe privately owned rights. Reference herein to any specific commercial product, process, or service by trade name, trademark, manufacturer, or otherwise, does not necessarily constitute or imply its endorsement, recommendation, or favoring by the United States Government or any agency thereof. The views and opinions of authors expressed herein do not necessarily state or reflect those of the United States Government or any agency thereof. 
Energy and Transportation Science Division

\title{
Environmental Quality and the U.S. Power Sector: Air Quality, Water Quality, Land Use and Environmental Justice
}

\author{
Emanuele Massetti, Georgia Institute of Technology \\ Marilyn A. Brown, Georgia Institute of Technology \\ Melissa Lapsa, Oak Ridge National Laboratory \\ Isha Sharma, Oak Ridge National Laboratory \\ James Bradbury, Department of Energy \\ Colin Cunliff, American Association for the Advancement of Science \\ Yufei Li, Georgia Institute of Technology
}

January 4, 2017

Prepared by

OAK RIDGE NATIONAL LABORATORY

Oak Ridge, TN 37831-6283

managed by

UT-BATTELLE, LLC

for the

US DEPARTMENT OF ENERGY

under contract DE-AC05-00OR22725 
This report is a DOE EPSA product prepared by Oak Ridge National Laboratory and is part of a series of "baseline" reports intended to inform the second installment of the Quadrennial Energy Review (QER 1.2). QER 1.2 will provide a comprehensive review of the nation's electricity system and covers the current state and key trends related to the electricity system, including generation, transmission, distribution, grid operations and planning, and end use. The baseline reports provide an overview of elements of the electricity system.

To help understand how the energy systems might develop into the future under Business as Usual (BAU) conditions QER 1.1 relied upon the U.S. Energy Information's Annual Energy Outlook (AEO) 2014 Reference Case. EPSA could not rely completely upon AEO for QER 1.2 as AEO 2016 was not completed and AEO 2015 did not include the Clean Power Plan. So the EPSA Base Case was developed and it aligns as closely as possible with AEO 2016 given the timing issues.

The EPSA Base Case scenario was constructed using EPSA-NEMS , a version of the same integrated energy system model used by EIA. The EPSA Base Case input assumptions were based mainly on the final release of AEO 2015, with a few exceptions as noted below, and then updated to include the Clean Power Plan and tax extenders. As with the AEO, the EPSA Base Case provides one possible scenario of base case energy sector demand, generation, and emissions from present day to 2040, and it does not include future policies that might be passed or future technological progress.

The EPSA Base Case input assumptions were based mainly on the final release of the AEO 2015, with a few updates that reflect current technology cost and performance estimates, policies, and measures. Assumptions from the EIA 2015 High Oil and Gas Resources Case were used; it has lower gas prices similar to those in AEO 2016. The EPSA Base Case achieves the broad emission reductions required by the Clean Power Plan. While states will ultimately decide how to comply with the Clean Power Plan, the EPSA Base Case assumes that states choose the mass-based state goal approach with new source complement and assumes national emission trading among the states, but does not model the Clean Energy Incentive Program because it is not yet finalized. The EPSA Base Case also includes the tax credit extensions for solar and wind passed in December 2015. In addition, the utility-scale solar and wind renewable cost and performance estimates have been updated to be consistent with EIA's AEO 2016. Carbon capture and storage (CCS) cost and performance estimates have also been updated to be consistent with the latest published information from the National Energy Technologies Laboratory. An EPSA Side Case was also completed, which has higher gas prices similar to those in the AEO 2015 Reference Case.

This activity was supported by a U.S. Department of Energy (DOE) American Association for the Advancement of Science (AAAS) Fellowship, administered by Oak Ridge Institute for Science and Education (ORISE). ORISE is managed by ORAU under DOE contract number DE-AC05-06OR23100.

\footnotetext{
a The version of the National Energy Modeling System (NEMS) used for the QER base case has been run by OnLocation, Inc., with input assumptions determined by EPSA. Since it uses a version of NEMS that differs from the one used by the U.S. Energy Information Administration (EIA), the model is referred to as EPSA-NEMS.
} 


\section{CONTENTS}

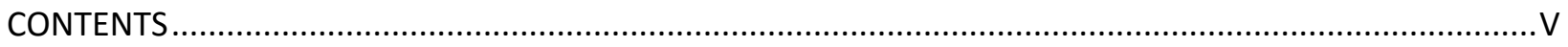

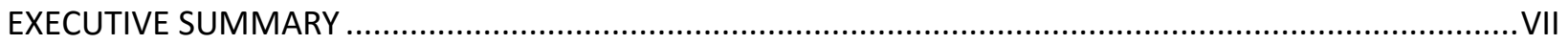

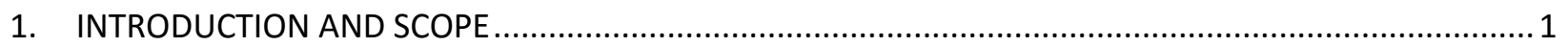

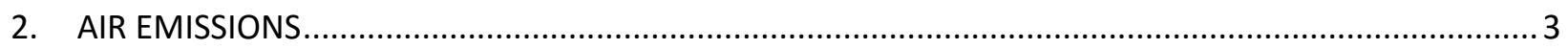

2.1 INTRODUCTION TO AIR EMISSIONS FROM POWER GENERATION …................................... 3

2.2 TRENDS OF AIR EMISSIONS FROM POWER GENERATION ............................................... 7

2.2.1 A Decomposition Analysis of Sulfur Dioxide Emission Reductions .............................12

2.2.2 Major Pollutants from Electric Power Generation: Geographic Distribution..............15

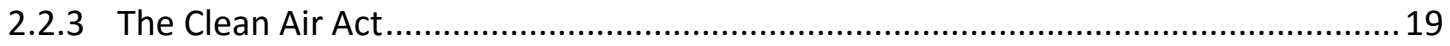

2.2.4 Technology and Innovation in Pollution Control ......................................................25

2.3 PROJECTIONS OF AIR EMISSIONS FROM ELECTRICITY GENERATION .....................................2

2.4 PHYSICAL IMPACTS OF AIR POLLUTION FROM POWER GENERATION ................................. 30

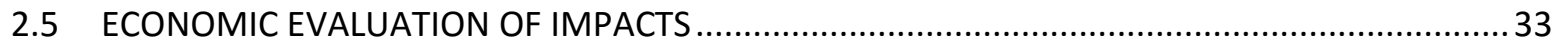

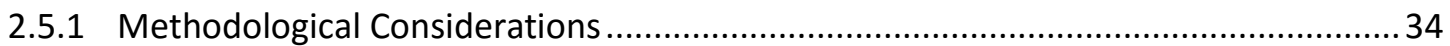

2.5.2 The Economic Cost of Air Pollution from Power Generation ......................................36

2.5.3 Air Quality Co-Benefits of $\mathrm{CO}_{2}$ Emissions Regulation ............................................. 45

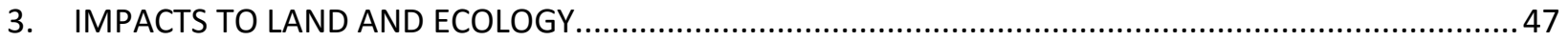

3.1 LAND-USE AND ECOLOGICAL IMPACTS OF POWER GENERATION .........................................4 48

3.1.1 Land Use Requirements of Power Generation ...................................................... 48

3.2 LAND USE AND ECOLOGICAL IMPACTS OF TRANSMISSION AND DISTRIBUTION ....................56

3.3 THE SITING AND PERMITTING OF ELECTRICITY INFRASTRUCTURE.....................................59

3.3.1 State Authorities for Infrastructure Siting ….......................................................60

3.3.2 Federal Authorities for Infrastructure Siting..........................................................6 61

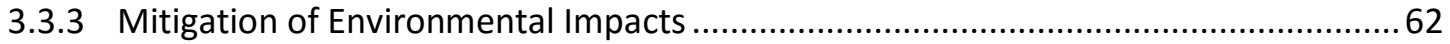

3.3.4 Federal Initiatives to Modernize Permitting and Review Processes............................63

3.4 OPPORTUNITIES TO FURTHER REDUCE LAND USE AND ECOSYSTEM IMPACTS ......................66

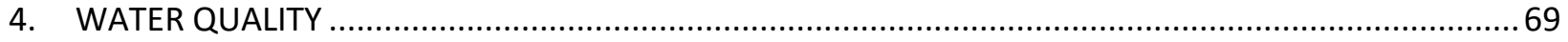

4.1 SOURCES OF WATER POLLUTION IN THE ELECTRIC POWER INDUSTRY ...............................69 
4.2 WATER QUALITY IMPACTS FROM POWER GENERATION

4.3 POLICIES REGULATING WATER QUALITY IMPACTS FROM STEAM ELECTRIC POWER

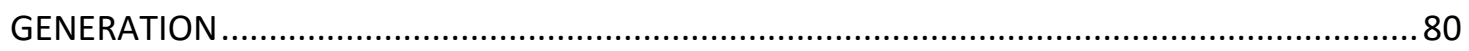

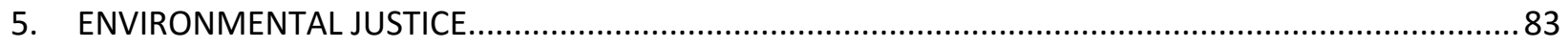

5.1 EJ CONSIDERATIONS/IMPACTS RELATED TO POWER GENERATION.................................... 84

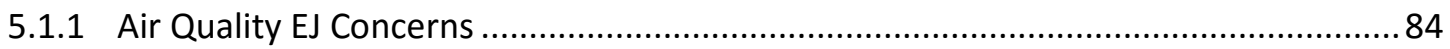

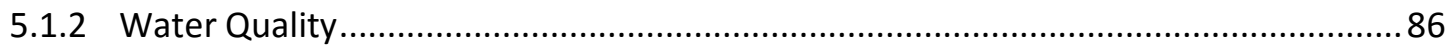

5.1.3 Climate Vulnerabilities to Environmental Justice Communities.................................89

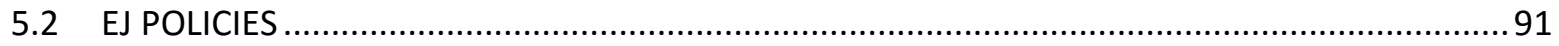

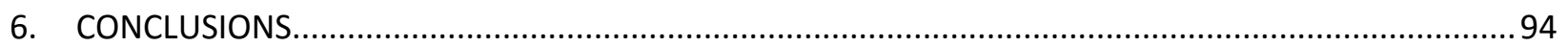

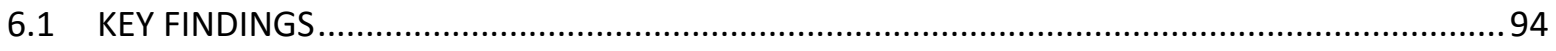

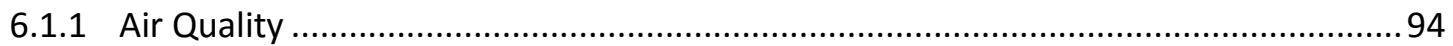

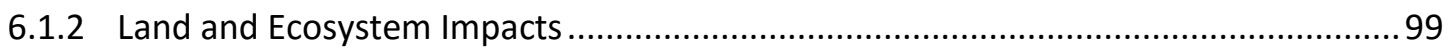

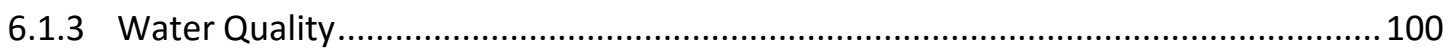

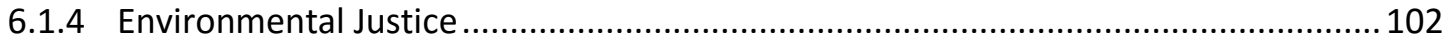

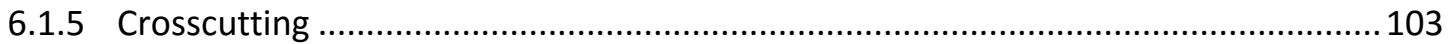

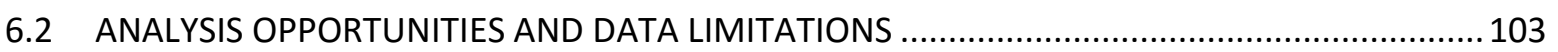

APPENDIX A. DECOMPOSITION ANALYSIS OF THE EVOLVING CONTRIBUTORS TO $\mathrm{SO}_{2}$ EMISSIONS

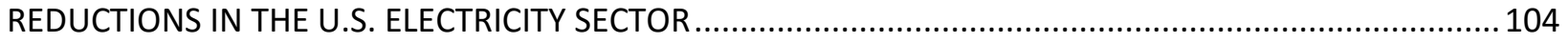

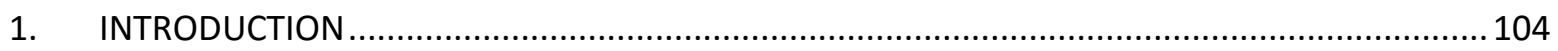

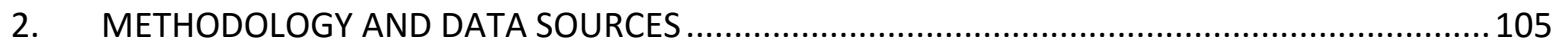

3. CONTRIBUTING FACTORS AND TRENDS OVER THE PAST 20 YEARS …............................... 106

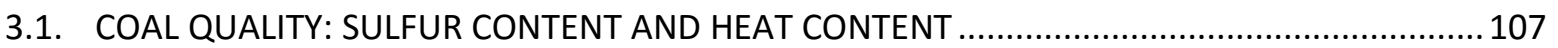

3.2. PLANT PERFORMANCES: HEAT RATE AND ELECTRICITY GENERATION.................................110

3.3. EMISSION CONTROL: DESULFURIZATION TECHNOLOGY AND REMOVAL ............................ 112

4. RESULTS: CONTRIBUTION OF EACH FACTOR TO FINAL REDUCTION ..................................113

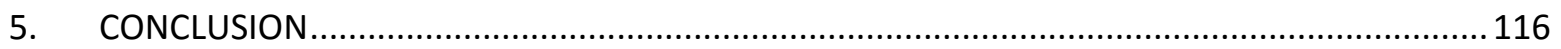

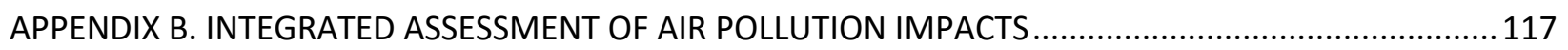

APPENDIX C. VALUATION METHODS OF ENVIRONMENTAL AND HEALTH IMPACTS ............................. 119

APPENDIX D SO ${ }_{2}$ AND NO EMISSIONS FROM DISTRIBUTED GENERATION .........................................123

APPENDIX E LIFE CYCLE IMPACTS OF ELECTRICITY GENERATION ................................................. 127 


\section{EXECUTIVE SUMMARY}

This baseline report summarizes key environmental quality issues associated with electricity generation, transmission, distribution, and end use in the United States. Its scope includes nongreenhouse gas air pollution (i.e., sulfur dioxide, nitrogen oxides, particulate matter and hazardous air pollutants), land use, water pollution, ecological impacts, human health, and environmental justice. The discussion characterizes both current impacts and recent trends, as well as assessments of key drivers of change. For example, the air emissions section includes a quantitative decomposition analysis of the drivers of change in sulfur dioxide emissions reductions from coal-fired power plants.

The report is divided into four topical sections: air emissions, land use and ecology, water quality, and environmental justice.

\section{Air Emissions}

Power generation from fossil fuels, biomass and waste contribute to emissions of air pollutants that adversely impact human health and the environment. ${ }^{b}$ Major air pollutants from the power sector include sulfur dioxide $\left(\mathrm{SO}_{2}\right)$, oxides of nitrogen $\left(\mathrm{NO}_{\mathrm{x}}\right)$, particulate matter, and mercury and other air toxics. These pollutants increase morbidity and the risk of mortality, reduce agricultural and timber productivity, deteriorate materials, reduce visibility, and harm ecosystems. However, air quality in the United States has been improving for decades, and additional improvements are expected in the coming years as a result of existing and expected market shifts, new regulations, and technology improvements.

Power plants are the largest source of $\mathrm{SO}_{2}$ emissions in the United States, accounting for $64 \%$ of economy-wide $\mathrm{SO}_{2}$ emissions in 2014. Power plants are also the main sources of mercury emissions and acid gases, accounting for $50 \%$ and over $75 \%$, respectively. Within the power sector, coal accounts for $98 \%$ of $\mathrm{SO}_{2}$ emissions, $94 \%$ of mercury emissions, $86 \%$ of $\mathrm{NO}_{\mathrm{x}}$ emissions, and $83 \%$ of fine particulate emissions. Natural gas accounts for $8 \%$ of $\mathrm{NO}_{\mathrm{x}}$ emissions and $12 \%$ of fine particulate emissions from the U.S. power sector and is not a major source of mercury or $\mathrm{SO}_{2}$ emissions.

Regulations promulgated under the Clean Air Act (CAA) have helped significantly reduce air pollution from the U.S. power sector. For example, nearly all coal-fired power plants in the

\footnotetext{
${ }^{\mathrm{b}}$ Non-combustion renewable energy technologies and nuclear power result in comparatively minor emissions of air pollutants, solely from upstream and downstream processes (e.g., raw material extraction and component manufacturing).
} 
United States have installed pollution controls for emissions of $\mathrm{SO}_{2}$ and $\mathrm{NO}_{x}$ to comply with CAA regulations. As a result of increased pollution control and other market trends (e.g., a decline in generation from coal), $2014 \mathrm{SO}_{2}$ emissions were 73\% below 1970 levels, and 2014 NO$_{\mathrm{x}}$ emissions were $57 \%$ lower than in 1970. Under the Mercury and Air Toxics Standards issued in 2011, power sector emissions of mercury in 2016 are projected to decline to $90 \%$ below 1990 levels as the rule took full effect in April 2016.

Figure ES-1. Historic and Projected $\mathrm{SO}_{2}, \mathrm{NO}_{x}$ and mercury air emissions from electricity generation: 2015-2040. ${ }^{123}$

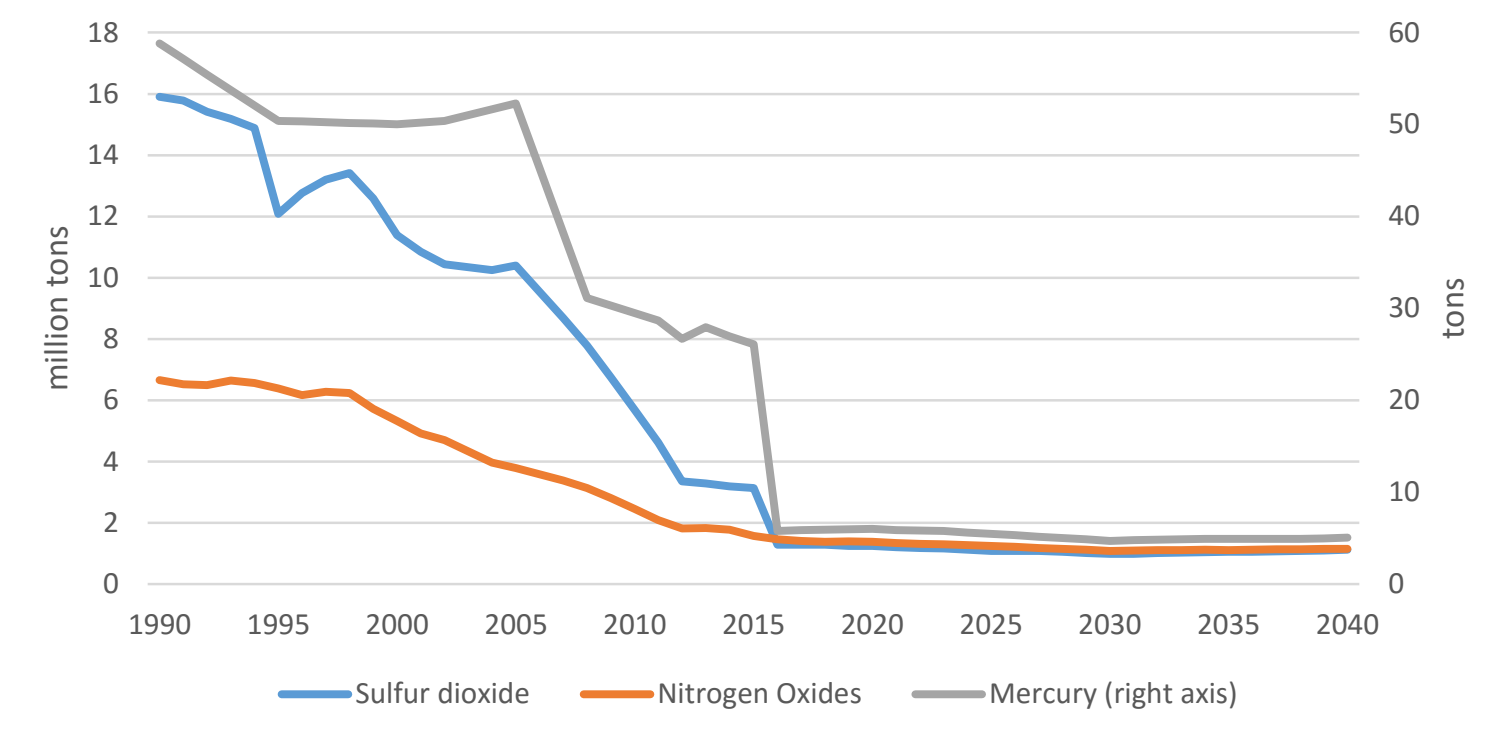

Sulfur dioxide and mercury emissions are projected to drop from 2015 to 2016 as affected coal units come into compliance with the MATS rule. After 2016, emissions largely track coal-fired generation.

\section{Impacts to Land and Ecology}

There is very limited published literature comparing life cycle land use and ecological impacts across all electricity generation technologies. The land use footprint of electricity infrastructures and associated operations have a range of direct impacts to ecosystems and society. The magnitude of these impacts depends on how the infrastructure affects endangered species, involves sensitive ecological areas, impacts cultural or historic resources, gives rise to visual or aesthetic concerns, or opens new areas to development.

Electricity infrastructure and operations can be detrimental to wildlife in a number of ways; however, technical and operational solutions are under active development. Fish and birds are impacted by air and water pollution from power plants. Nuclear and fossil fuel-related bird deaths have been estimated to be comparable to deaths caused by wind power, per kWh generated. Avian mortalities are caused by wind turbines and concentrated solar plants; impacts vary significantly by region and facility. However, bird mortalities due to collisions with 
wind turbines are much lower compared to bird mortalities from collisions with other engineered structures such as buildings.

A key finding from the first installment of the Quadrennial Energy Review is that while expanding transmission and distribution infrastructure can pose environmental challenges, building new infrastructure can help to enable significant net positive environmental outcomes. ${ }^{4}$ For this reason, agencies across the federal government are engaged in several initiatives to modernize the Federal government's role with respect to electric transmission permitting and project review. ${ }^{5}$ These initiatives are designed to support better decision making while enabling better outcomes for public health, the environment, safety and communities.

\section{Water Quality}

Certain types of power generation facilities generate pollution that contributes to water quality problems through a variety of mechanisms. Air emissions of mercury, $\mathrm{NO}_{x}$ and $\mathrm{SO}_{2}$ impact water quality through wet deposition. Additionally, steam electric plants may generate wastewater streams that can impact ground water and surface water quality. The plants generate wastewater pollution in the form of chemical pollutants and thermal pollution (heated water) from their water treatment, power cycle, ash handling, and air pollution control systems, as well as from coal piles, yard and floor drainage, and other miscellaneous wastes.

Steam electric power plants are the largest source of toxic discharges to water bodies, accounting for about $30 \%$ of all toxic discharges from industrial sources in the United States. Wastewater pollutants include both toxic and bioaccumulative pollutants such as arsenic, lead, mercury, selenium, chromium, and cadmium, as well as significant levels of chloride, nutrients, and other dissolved and suspended solids. Impacts from these pollutants fall into several broad categories, including increased health risks, impacts to surface and ground water quality, and other environmental impacts. Major impacts to human health-primarily from exposure to contaminated fish tissue-include lowered IQ in children and infants, increased incidence of cardiovascular disease in adults, and increased incidence of cancer.

New Clean Water Act regulations are projected to reduce pollutant discharges from power plants by over 95 percent for copper, lead, mercury, nickel, selenium, thallium, and zinc and over 90 percent for arsenic and cadmium. Across all pollutants, toxic discharges from power plants are projected to decline by 63 percent by the time the new regulation takes full effect in 2023. 


\section{Environmental Justice}

Environmental justice (EJ) is the fair treatment and meaningful involvement of all people regardless of race, color, national origin, or income with respect to the development, implementation, and enforcement of environmental laws, regulations, and policies. Environmental justice is achieved when everyone enjoys the same degree of protection from environmental and health hazards and equal access to the decision-making process to have a healthy environment in which to live, learn and work. EJ concerns related to the electric power industry include the impacts to air and water quality, the impacts of climate change, and equal access to affordable electric bills.

Populations of concern may face different levels of vulnerability to the air quality, water quality and climate impacts from power generation because of differences in exposure or inherent sensitivity to pollution or because of reduced capacity to adapt to the impacts of pollution. For example, a greater percentage of minorities and people living below the poverty level live within a three-mile radius of coal- and oil-fired power plants, compared to the U.S. population overall. Additionally, existing health disparities and other inequities in these communities increase their vulnerability to the health effects of degraded air and water quality and climate change. 


\section{INTRODUCTION AND SCOPE}

This report is one of a series of environmental baseline documents prepared on behalf of the U.S. Department of Energy Office of Energy Policy and Systems Analysis (EPSA) to frame the environmental questions posed by the modern grid. It summarizes key environmental quality issues associated with electricity generation, transmission, distribution, and end use. The focus of this report is on the non-greenhouse gas (GHG) air pollution (sulfur dioxide, nitrogen oxides, particulate matter and hazardous air pollutants), land use, water pollution, environmental justice, human health, and ecological impacts of the U.S. electricity system. The scope includes a discussion of important issues and recent trends, as well as assessments of key drivers of change. For example, the air emissions section includes a quantitative decomposition analysis on the drivers of change in sulfur dioxide emissions from coal-fired power plants.

In addition to the introduction and concluding sections, this volume is organized into four topical sections: air emissions, land and ecology, water quality and environmental justice. In examining these issues, this work summarizes and synthesizes the findings of relevant journal articles, reports, and books published since the year 2000.

Other environmental issues are addressed by other volumes of the environment baseline. For instance, while this volume discusses water quality issues that result from pollution from power plants, this volume will not address the issue of water use (e.g., cooling water withdrawal and consumption, as well as thermal pollution impacts, are covered in the Energy Water Nexus volume). This baseline will also not cover upstream effects-such as mining and construction environmental damage-which are out of scope for this report. Solid waste from power generation ${ }^{c}$ will also not be discussed because that issue is covered in another environmental baseline report. ${ }^{6}$ Finally, GHG emissions from the power sector are covered in a separate baseline report. ${ }^{7}$ It is noted, however, that some $\mathrm{CO}_{2}$ mitigation measures may also reduce other types of air pollution.

More than 6,500 power plants operate in the U.S. power sector, ${ }^{d}$ generating 3,937 Terawatthours (TWh) of electricity in 2014. ${ }^{8}$ In 2014, the U.S. generation mix included coal (39\%), natural gas (28\%), nuclear (19\%), hydropower $(6 \%)$, and non-hydro renewable sources $(7 \%){ }^{9}$ This power generation mix is rapidly changing as a result of evolving market, technology,

\footnotetext{
${ }^{\mathrm{c}}$ Fossil fuel combustion can result in solid waste such as coal ash and scrubber slurries, and nuclear power generation produces radioactive waste that must be stored and eventually disposed of properly.

${ }^{d}$ Includes electric utilities and independent power producers.

e An additional 162 TWh of electricity was generated at distributed generation facilities in the commercial and industrial sectors in 2014. Distributed generation refers to electricity that is consumed on-site, without being sold through the bulk power system. Emissions from distributed generation are addressed in Appendix D.
} 
resource, and policy trends (Figure 1.1). More information about electric generation technologies and the changing generation mix is addressed in the Generation baseline report. ${ }^{10}$

Figure 1.1. Power sector electricity generation by fuel: total and share. ${ }^{11}$
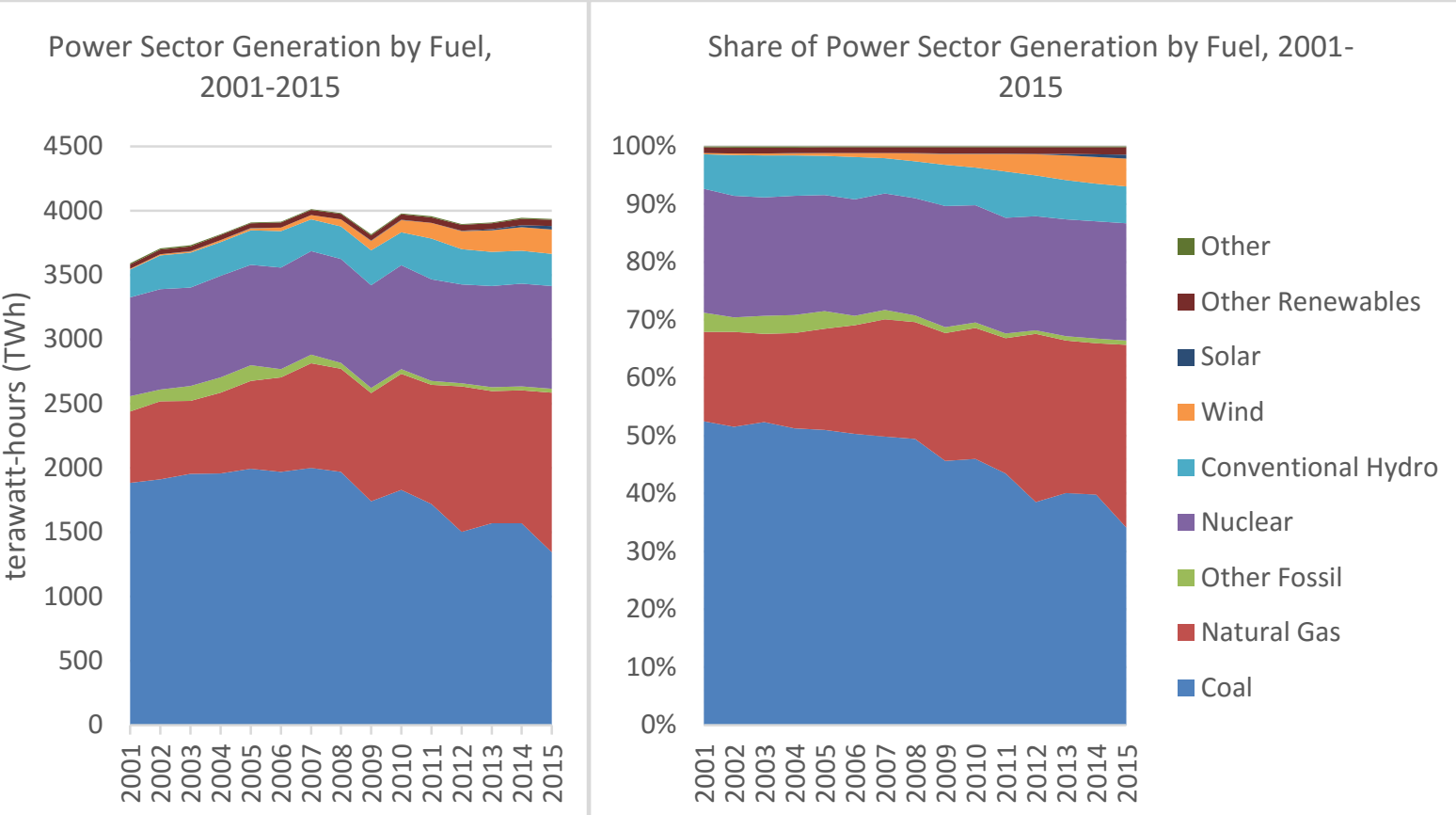

Coal has traditionally accounted for the largest share of electricity generation, followed by natural gas and nuclear power. In recent years, the share of generation from coal has declined, while electricity from natural gas and renewable energy has increased.

The impact of these power plants on air quality, water quality, land use, human health and ecosystems is a function of the fuel used to generate electricity, the type and age of generation equipment, the nature and extent of pollution control employed, the geographic location of the infrastructure, and meteorological and other factors. 


\section{AIR EMISSIONS}

Power generation from fossil fuels, biomass and waste contribute to emissions of criteria air pollutants (CAPs) and their precursors, as well as hazardous air pollutants (HAPs). ${ }^{12}$ Criteria air pollutants are ubiquitous air pollutants that are regulated under the national ambient air quality standards (NAAQS) program. Hazardous air pollutants, also known as toxic air pollutants, are those known to cause cancer and other serious health effects, such as reproductive effects or birth defects, or adverse environmental effects. These pollutants are regulated under the national emission standards for hazardous air pollutants (NESHAPs) program. Electricity generation is also a major source of greenhouse gas (GHG) pollution, which traps heat in the atmosphere. Power sector GHG emissions are addressed in a separate Environmental baseline volume. ${ }^{13}$

\subsection{INTRODUCTION TO AIR EMISSIONS FROM POWER GENERATION}

Emissions of CAPs and HAPs increase morbidity and the risk of mortality, reduce agricultural and timber productivity, deteriorate materials, reduce visibility and harm ecosystems. ${ }^{14}$ Major air pollutants from the power sector include sulfur dioxide $\left(\mathrm{SO}_{2}\right)$, oxides of nitrogen $\left(\mathrm{NO}_{\mathrm{x}}\right)$, particulate matter $\left(\mathrm{PM}_{10}\right.$ and $\left.\mathrm{PM}_{2.5}\right){ }^{f}$ and mercury and other air toxics. CAPs such as $\mathrm{SO}_{2}$ and $\mathrm{NO}_{\mathrm{x}}$ are subject to national air quality standards established by the EPA that are enforced at the state level. HAP emissions such as mercury and other air toxics are regulated by technologybased standards that are enforced at a facility level. Table 2.1 provides an overview of air emissions and their legal definitions, under the CAA.

Sulfur dioxide $\left(\mathrm{SO}_{2}\right)$ has direct environmental impacts ${ }^{15}$ and it is a precursor of fine particle pollution (also known as fine particulate matter or $\mathrm{PM}_{2.5}$ ), the leading cause of increased risk of morbidity and mortality from air pollution. ${ }^{16}$ Together with nitrogen oxides, sulfur dioxide is the major cause of acid rain. ${ }^{17}$

Nitrogen oxides (NOx) are a group of gases that are highly reactive. Nitrogen oxides contribute to the formation of ground-level ozone and particulate matter, which cause respiratory and other negative health impacts. ${ }^{18}$

Particulate matter (PM) is a complex mixture of extremely small particles and liquid droplets, including acids (such as nitrates and sulfates), organic chemicals, metals, and soil or dust particles. Once inhaled, these particles can affect the heart and lungs and cause serious health effects. ${ }^{19} \mathrm{PM}_{10}$ consists of particles 10 micrometers or smaller in diameter. $\mathrm{PM}_{2.5}$, also called

\footnotetext{
${ }^{\mathrm{f}} \mathrm{PM}$ refers to particulate matter with a diameter less than or equal to $d$, measured in micrometers. So $\mathrm{PM} 10$ consists of particles 10 micrometers or smaller in diameter.
} 
fine particulate matter, consists of particles 2.5 micrometers or smaller in diameter. ${ }^{g}$ Power generation is both a direct source of $\mathrm{PM}$ and an indirect source because $\mathrm{SO}_{2}$ and $\mathrm{NO}_{\mathrm{x}}$ can be chemically transformed in the atmosphere into fine particulate matter. ${ }^{20}$

Carbon monoxide (CO) is a by-product of combustion that, if inhaled, reduces the amount of oxygen transported to the organs and likely increases cardiovascular morbidity. ${ }^{21}$

Volatile organic compounds (VOC) are a large array of chemicals emitted as gases from a large number of solids and liquids and have adverse health impacts. VOCs are regulated precursors involved in formation of pollutants for which national ambient air quality standards have been established, but power generation is not a major source of VOCs. VOCs are, however, one of the primary contributors to the formation of ground level ozone.

Ground level ozone is a particularly harmful secondary pollutant for which national ambient air quality standards have been established. Ozone is formed by chemical reactions between VOCs and $\mathrm{NO}_{\mathrm{x}}$ stimulated by sunlight. ${ }^{22}$ Both VOCs and NOx are emitted as a result of fossil power generation, among other sources. ${ }^{\text {h }}$ Elevated concentrations of ground-level ozone have a variety of negative effects on human health and welfare (e.g., crop productivity). ${ }^{23}$

HAPs include 187 hazardous compounds known or suspected to cause cancer or other serious health effects. In particular, mercury and other air toxics' that are emitted as a result of electricity generation from fossil fuels are regulated as HAPs under the Clean Air Act. ${ }^{24}$

\footnotetext{
g Particles that are between 2.5 and $10 \mu \mathrm{m}$ in size are denoted with the symbol PM10-2.5.

${ }^{\mathrm{h}}$ Other sources of VOCs include anthropogenic sources (paints and coating, gasoline, building materials, cosmetic and cleaning products) and biological sources (plants, animals and fungi).

${ }^{\prime}$ In addition to mercury ( $\left.\mathrm{Hg}\right)$, HAP includes other metals such as arsenic (As), cadmium (Cd), chromium ( $\mathrm{Cr}$ ) and nickel (Ni). Hazardous acid gases-including hydrochloric acid $(\mathrm{HCl})$ and hydrofluoric acid $(\mathrm{HF})$-are also regulated HAP from power plants.
} 
Table 2.1. Air emissions overview.

\begin{tabular}{|c|c|c|}
\hline $\begin{array}{l}\text { Regulated } \\
\text { Emissions }\end{array}$ & Examples & Definition History \\
\hline $\begin{array}{l}\text { Criteria Air } \\
\text { Pollutants (CAP) }\end{array}$ & $\begin{array}{l}\text { Sulfur dioxide; Nitrogen Oxides; } \\
\text { Carbon Monoxide; Particulate } \\
\text { Matter; Ozone; and Lead. }\end{array}$ & $\begin{array}{l}\text { Under Title I, the CAA requires EPA to set national ambient } \\
\text { air standards for each of the criteria pollutants, based on } \\
\text { health risk studies. }\end{array}$ \\
\hline $\begin{array}{l}\text { Hazardous Air } \\
\text { Pollutants (HAPs) }\end{array}$ & $\begin{array}{l}\text { Carcinogenic HAP, including } \\
\text { benzene, naphthalene, 1,3- } \\
\text { butadiene, Polycyclic aromatic } \\
\text { hydrocarbons (PAH). Non- } \\
\text { carcinogenic HAP, including } \\
\text { Hydrogen Fluoride (HF) and } \\
\text { Hydrogen Cyanide (HCN). Persistent } \\
\text { bioaccumulative HAPs include } \\
\text { mercury and other heavy metals. } \\
\text { These HAP can cause damage to } \\
\text { multiple organ systems. Mercury is } \\
\text { best known as a neurotoxin. }\end{array}$ & $\begin{array}{l}\text { Toxic Air Pollutants, also called "hazardous air pollutants," } \\
\text { are regulated under Title III of the Clean Air Act Amendments } \\
\text { of } 1990 \text {. Congress established a list of nearly } 190 \text { hazardous } \\
\text { compounds known or suspected to cause cancer or other } \\
\text { serious health effects. Since the HAP sources are often } \\
\text { specific to industry processes or equipment types, EPA has } \\
\text { identified categories of major industrial sources of these } \\
\text { toxic chemicals and developed emission standards for each. } \\
\text { These standards are based on the maximum reduction in } \\
\text { emissions achievable taking into consideration costs and } \\
\text { certain other factors. }\end{array}$ \\
\hline $\begin{array}{l}\text { Volatile Organic } \\
\text { Compounds (VOC) }\end{array}$ & $\begin{array}{l}\text { Organic compounds that are } \\
\text { photochemically reactive }\end{array}$ & $\begin{array}{l}\text { Volatile organic compounds (VOCs) are one of two main } \\
\text { ingredients in forming ground-level ozone along with } \mathrm{NO}_{\mathrm{x}} \text {. } \\
\text { The emission of VOCs can contribute to ozone levels that } \\
\text { may exceed air quality standards set by the EPA. }\end{array}$ \\
\hline Other Pollutants & Hydrogen Sulfide $\left(\mathrm{H}_{2} \mathrm{~S}\right)$ & $\begin{array}{l}\text { Hydrogen Sulfide emissions must be reported to the Toxics } \\
\text { Release Inventory (TRI) by specific industries each year. } \\
\text { Industrial processes such as food processing, coke ovens, } \\
\text { petroleum refineries and other industries produce this } \\
\text { compound. }\end{array}$ \\
\hline $\begin{array}{l}\text { Greenhouse Gases } \\
\text { (GHGs) }\end{array}$ & $\begin{array}{l}\text { Carbon Dioxide }\left(\mathrm{CO}_{2}\right) \\
\text { Methane }\left(\mathrm{CH}_{4}\right) \\
\text { Nitrous Oxide }\left(\mathrm{N}_{2} \mathrm{O}\right) \\
\text { Hydrofluorocarbons (HFCs) }\end{array}$ & $\begin{array}{l}\text { In 2009, under Section 202(a) authorities of the Clean Air } \\
\text { Act, EPA made a finding that six well-mixed GHGs constitute } \\
\text { a threat to public health and welfare, and that the combined } \\
\text { emissions from motor vehicles cause and contribute to } \\
\text { climate change. The EPA has set standards for GHG } \\
\text { emissions for certain mobile and stationary sources. }\end{array}$ \\
\hline
\end{tabular}

Air pollution from electricity generation depends on a number of factors, including the fuel type, the quality of the fuel, combustion technology and the efficiency of the electric generating unit, and the availability of pollution controls. The majority of electricity-related emissions come from the combustion of fossil fuels, including coal, natural gas, and petroleum products. Smaller amounts of pollutants are also emitted from biomass and other sources. In general, other renewable sources - including hydropower, geothermal, wind, and solar-and nuclear power emit little or no emissions during electricity generation. Figure 2.1 shows the electricity generation mix for the power sector and power sector emissions from the National Emissions Inventory (NEI) for 2011. While coal accounted for only 43.5 percent of electricity generation in 2011, it was responsible for 93 percent of power sector emissions of criteria air pollutants (such as $\mathrm{SO}_{2}$ and $\mathrm{NO}_{x}$ ) and 94 percent of hazardous air pollutants such as mercury. 
Figure 2.1. Power Sector Generation and Emissions of Select Criteria Air Pollutants and Mercury by Fuel, 2011.25 $26 \mathrm{j} \mathrm{k}$

Power Sector Generation by Fuel, 2011
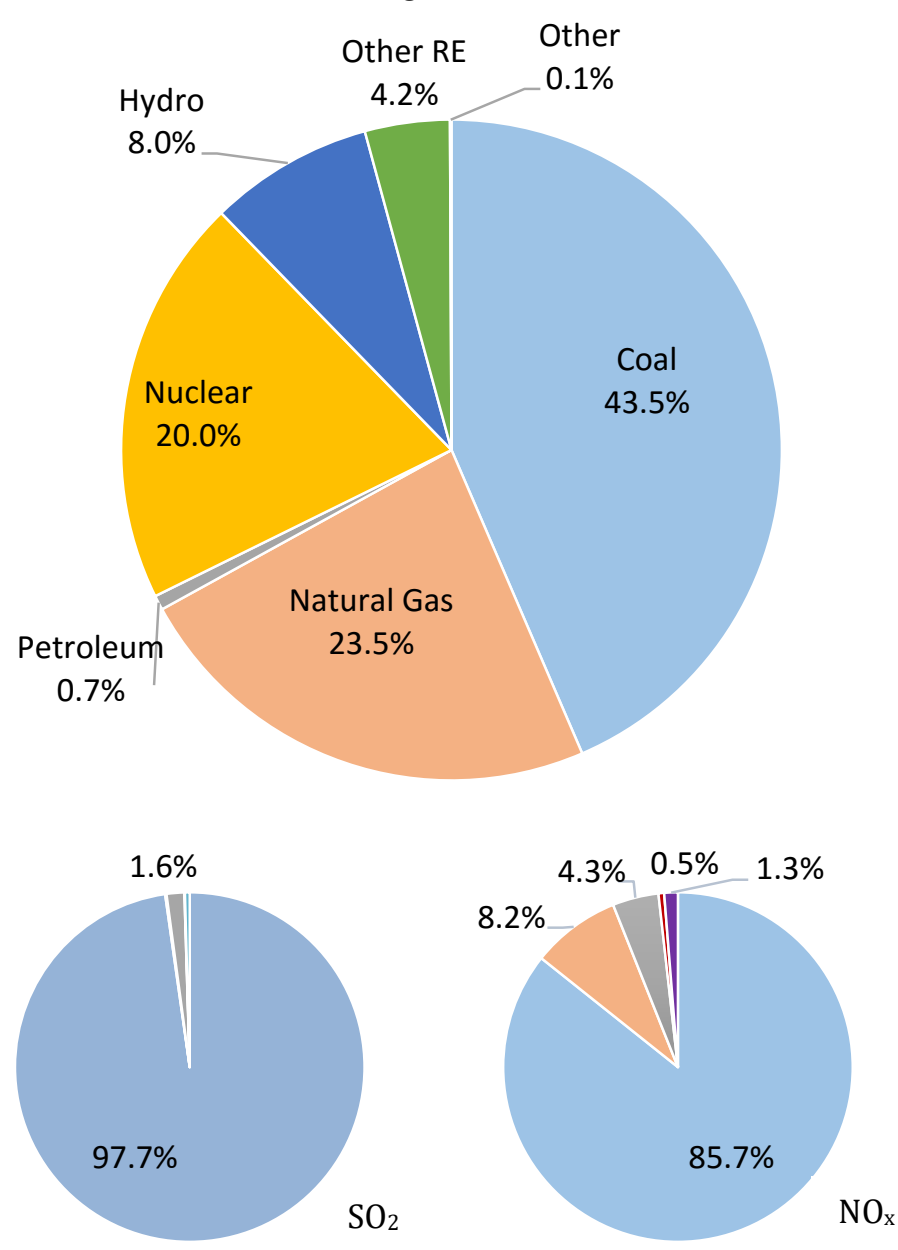

Emissions by Fuel, 2011
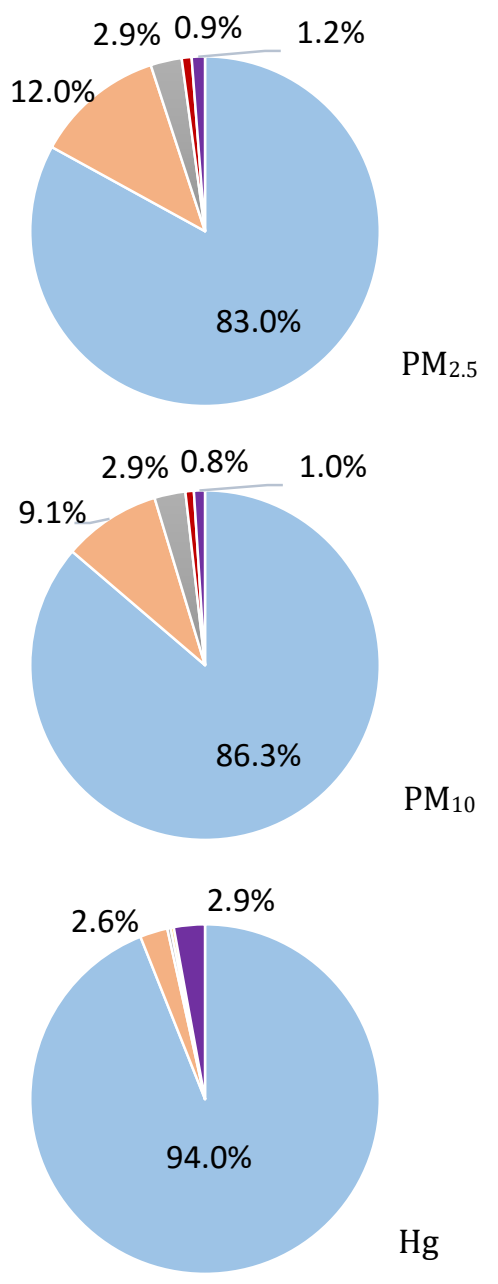

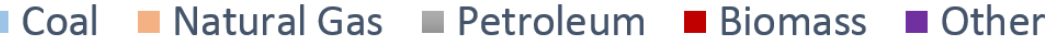

In 2011 , coal accounted for $43.5 \%$ of electricity generation but accounted for the vast majority of air pollutants, including $97.5 \%$ of $\mathrm{SO}_{2}$ emissions and $85.7 \%$ of $\mathrm{NO}_{x}$ emissions.

Note: Generation by fuel is displayed for coal, natural gas, petroleum, nuclear, conventional hydropower, other renewable energy (Other RE), and other sources. Other RE includes geothermal, wind, solar,

\footnotetext{
${ }^{\mathrm{j}}$ The National Emissions Inventory is updated every three years. At the time of publication, the NEl only included emissions by fuel data through 2011. The 2014 NEl v1, which will include emissions-by-fuel data through 2014, is scheduled for release in August 2016.

${ }^{\mathrm{k}}$ Note that the generation mix and emissions profile has changed in subsequent years as a result of market forces and policy drivers. In 2015, the share of power sector electricity generated from coal fell to $34.2 \%$, while the share of power sector generation from natural gas and renewables increased to $31.6 \%$ and $13.2 \%$, respectively. For more information on trends in electricity generation, see the Electricity Generation baseline.
} 
biomass, and waste. In 2011, biomass (shown in red on the emissions pie charts) accounted for $0.7 \%$ of electricity generation and is included in the $4.2 \%$ of generation from Other RE in the generation pie chart.

The emission rate for different types of fuels is another key indicator of the environmental impact of electricity generation and is defined as the ratio of the amount of pollutant emitted (measured in kilograms or pounds)' per the amount of electricity generated (measured in megawatt-hours). The emission rate depends on the quality and type of fuel, the heat content of the fuel, the thermal efficiency of electricity production, and the availability of pollution controls. Table 2.2 displays the emissions and emission rates by fuel for $\mathrm{SO}_{2}$ and $\mathrm{NO}_{x}$ emissions in 2011.

Table 2.2. Power Sector $\mathrm{SO}_{2}$ and $\mathrm{NO}_{x}$ Emissions and Emission Rates by Fuel, 2011. ${ }^{27}$

\begin{tabular}{|c|c|c|c|c|c|}
\hline & $\begin{array}{l}\text { Generation } \\
\text { (MWh) }\end{array}$ & $\begin{array}{l}\mathrm{SO}_{2} \text { Emissions } \\
\text { (short tons) }\end{array}$ & $\begin{array}{l}\mathrm{SO}_{2} \text { Emission rate } \\
\left.\text { (Ib SO} \mathrm{S}_{2} / \mathrm{MWh}\right)\end{array}$ & $\begin{array}{l}\text { NO }_{x} \text { Emissions } \\
\text { (short tons) }\end{array}$ & $\begin{array}{l}\mathrm{NO}_{\mathrm{x}} \text { Emission rate } \\
\left.\text { (Ib NO } \mathrm{NO}_{\mathrm{x}} / \mathrm{MWh}\right)\end{array}$ \\
\hline Coal & $1,717,890,732$ & $4,521,128$ & 5.26 & $1,791,446$ & 2.09 \\
\hline Natural Gas & $926,290,376$ & 5,708 & 0.01 & 172,235 & 0.37 \\
\hline Oil & $28,202,160$ & 75,756 & 5.37 & 89,103 & 6.32 \\
\hline Biomass & $26,722,118$ & 2,355 & 0.18 & 10,991 & 0.82 \\
\hline Other & $1,247,838,237$ & 20,349 & 0.03 & 26,341 & 0.04 \\
\hline
\end{tabular}

Emission rates shown are effective average emission rates based on the 2011 generation mix in the power sector, and include any pollution controls that were in operation as of 2011. In 2011, the average emission rates for coal were $5.26 \mathrm{lb} \mathrm{SO}_{2} / \mathrm{MWh}(2.39 \mathrm{~kg} \mathrm{SO} / \mathrm{MWh})$ and $2.09 \mathrm{lb} \mathrm{NO} \times / \mathrm{MWh}(0.95 \mathrm{~kg}$ $\mathrm{NO} \times / \mathrm{MWh}$ ). Oil has the highest emission rates for $\mathrm{SO}_{2}$ and $\mathrm{NO}_{x}$ but makes up a relatively small portion of the overall generation mix. Other electricity generation includes nuclear power, conventional hydropower, wind, solar, geothermal, and other gases.

\subsection{TRENDS OF AIR EMISSIONS FROM POWER GENERATION}

Table 2.3 displays long-term data on $\mathrm{SO}_{2}$ and $\mathrm{NO}_{x}$ emissions from U.S. electric utilities. The table also displays the relative contributions of the electric power sector to total emissions for each pollutant and emissions per MWh of electricity generation.

\footnotetext{
I One kilogram weighs 2.205 pounds, so emission rates in $\mathrm{kg} / \mathrm{MWh}$ can be converted to emission rates in $\mathrm{lb} / \mathrm{MWh}$ by multiplying by a factor of 2.205 .
} 
Table 2.3. $\mathrm{SO}_{2}$ and $\mathrm{NO}_{x}$ emissions of electric utilities. ${ }^{29} 30$

\begin{tabular}{|c|c|c|c|c|c|c|c|c|}
\hline & \multicolumn{4}{|c|}{ Emissions (thousands of tons) } & \multicolumn{4}{|c|}{$\begin{array}{l}\text { Electric power emissions } \\
\text { as share of total US emissions }\end{array}$} \\
\hline & 1970 & 1990 & 2010 & 2014 & 1970 & 1990 & 2010 & 2014 \\
\hline $\mathrm{SO}_{2}$ & 17,398 & 15,909 & 5,696 & 3,195 & $55.7 \%$ & $68.9 \%$ & $73.7 \%$ & $64.0 \%$ \\
\hline \multirow[t]{3}{*}{$N O_{x}$} & 4,900 & 6,663 & 2,458 & 1,776 & $18.2 \%$ & $26.1 \%$ & $16.6 \%$ & $14.3 \%$ \\
\hline & \multicolumn{4}{|c|}{$\begin{array}{l}\text { Ibs per MWh } \\
\text { (all fuels) }\end{array}$} & \multicolumn{4}{|c|}{$\begin{array}{c}\text { lbs per MWh } \\
\text { (excluding renewables and nuclear) }\end{array}$} \\
\hline & 1970 & 1990 & 2010 & 2014 & 1970 & 1990 & 2010 & 2014 \\
\hline $\mathrm{SO}_{2}$ & 22.7 & 11.0 & 2.87 & 1.62 & 27.6 & 30.8 & 6.61 & 5.11 \\
\hline$N O_{x}$ & 6.40 & 4.6 & 1.24 & 0.90 & 7.77 & 12.9 & 2.85 & 2.84 \\
\hline
\end{tabular}

Sulfur dioxide and $\mathrm{NO}_{\mathrm{x}}$ emissions from the U.S. electric power sector have sharply declined in recent decades. $\mathrm{SO}_{2}$ emissions in 2014 are 73\% lower than in 1970, and 2014 NOx emissions are $57 \%$ lower than $1970 \mathrm{NO}_{x}$ emissions. ${ }^{31} 32$ The rate of reductions in $\mathrm{SO}_{2}$ and $\mathrm{NO}_{\mathrm{x}}$ emissions increased rapidly after passage of the 1990 Clean Air Act amendments. ${ }^{33} 34$ Direct emissions of $\mathrm{PM}_{2.5}$ and $\mathrm{PM}_{10}$ have declined by about $60 \%$ during the past ten years, in line with recent trends of $\mathrm{SO}_{2}$ and $\mathrm{NO}_{x}$ emissions (Figure 2.1). The declines have occurred even as electricity generation has increased (Table 2.3 and Figure 2.2). Section 2.2.2 provides a more detailed discussion of the trend of $\mathrm{SO}_{2}$. 
Figure 2.2. Trend in selected criteria air pollutant emissions from fossil fuel combustion by electric utilities, 2002-2014. ${ }^{35} 36 \mathrm{~m}$

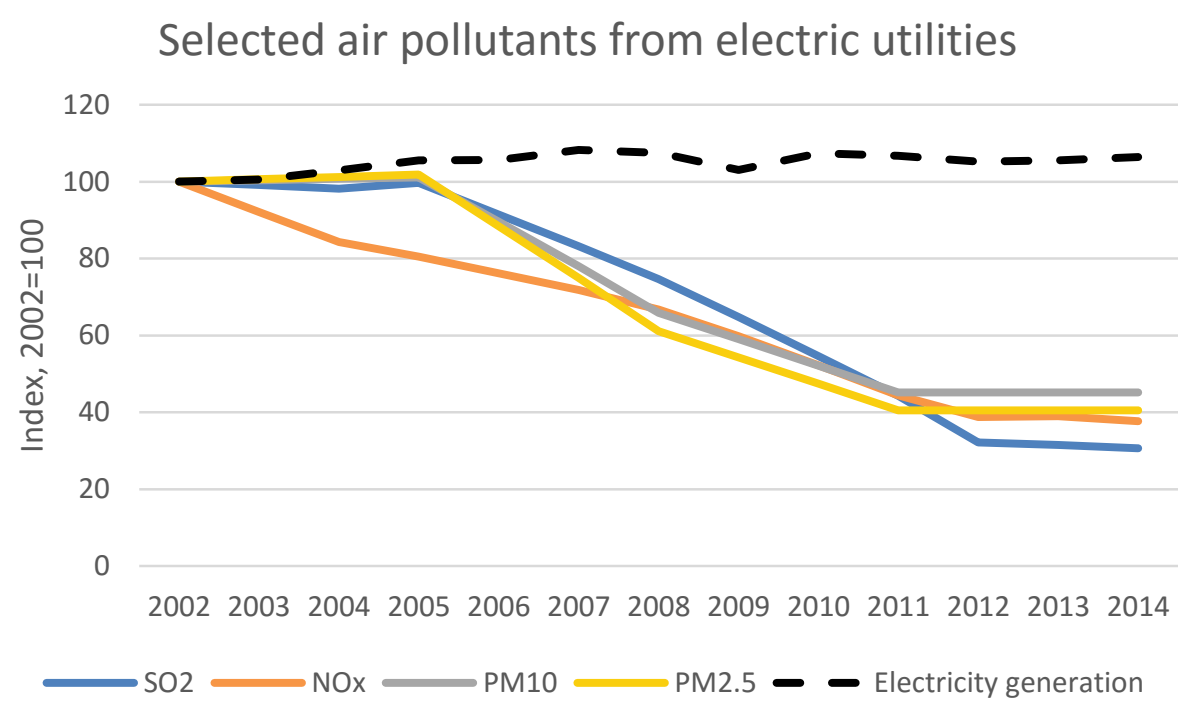

Power sector emissions of particulate matter, sulfur dioxide, and nitrogen oxides have declined in the range of 55 to 65 percent since 2002.

${ }^{\mathrm{m}} \mathrm{SO}_{2}$ and $\mathrm{NO}_{x}$ emissions data have been updated through 2014 using data from EPA's Clean Air Markets Database. However, particulate emissions ( $\mathrm{PM}_{10}$ and $\mathrm{PM}_{2.5}$ ) are presumed constant at 2011 levels and may be updated with the public release of the 2014 National Emissions Inventory v1, currently scheduled for August 15, 2016. 
Figure 2.3. Emissions by Sector for Select Criteria Air Pollutants, 2002-2014. ${ }^{37}$

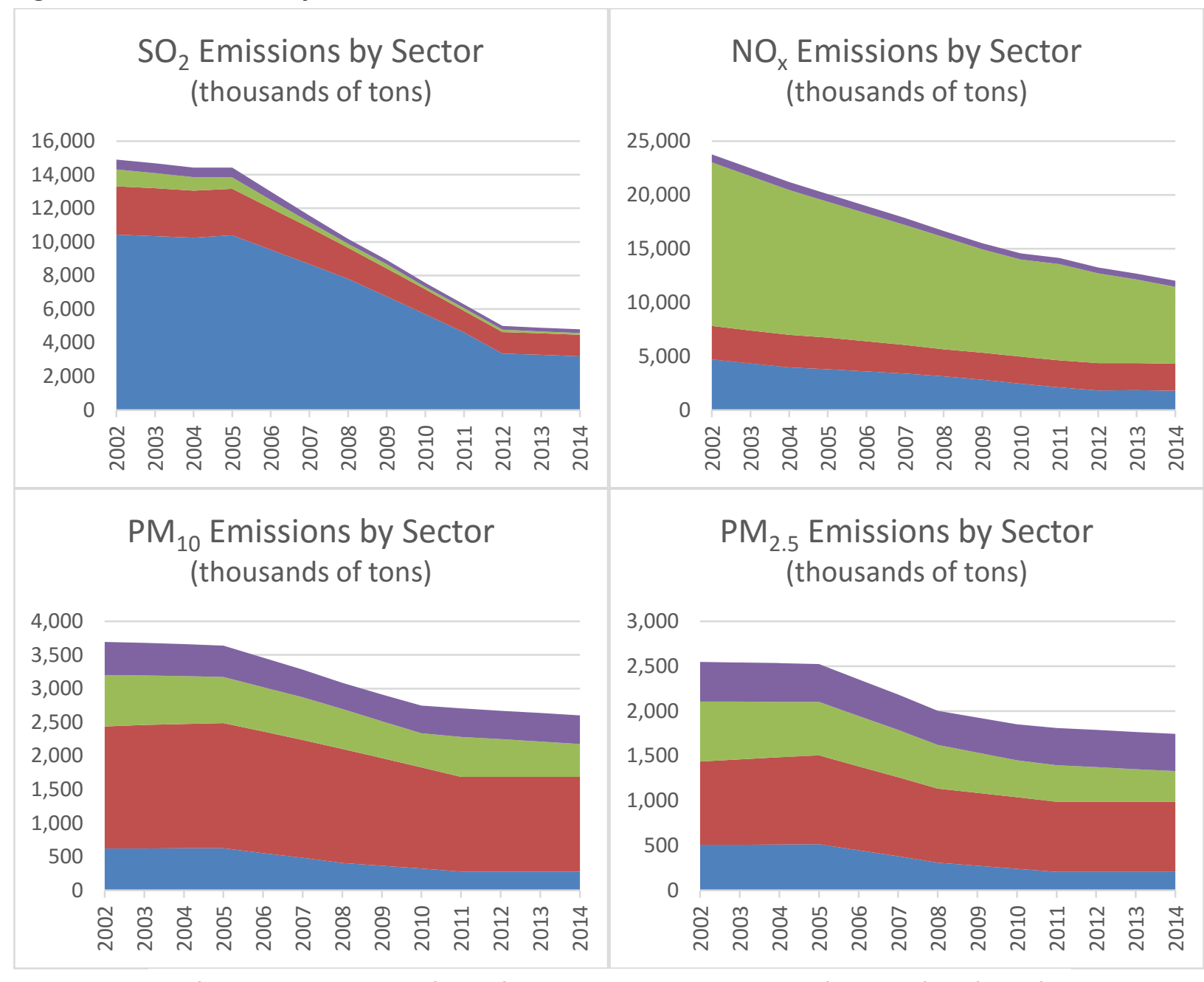

\section{घlectric Power $\square$ Industrial $\square$ Transportation $\quad$ Other Fossil Fuel Comb.}

Electric utilities are the largest source of $\mathrm{SO}_{2}$ emissions, accounting for $64 \%$ of $\mathrm{SO}_{2}$ emissions across all sources in 2014. Electric utilities contribute a smaller share of total $\mathrm{NO}_{x}(14 \%), \mathrm{PM}_{2.5}(6.9 \%)$, and $\mathrm{PM}_{10}$ $(1.4 \%)$ emissions.

Note: These graphs exclude sources categorized as "Miscellaneous" in the Air Pollutant Emissions Trends Data. While not a significant contributor of $\mathrm{SO}_{2}$ and $\mathrm{NO}_{x}$ emissions, "Miscellaneous" sources, including wildfires and dust, contribute a majority of all $\mathrm{PM}_{10}(87 \%)$ and $\mathrm{PM}_{2.5}$ (71\%) emissions monitored by EPA.

Electric utilities also emit modest quantities of $\mathrm{CO}, \mathrm{VOC}$, and $\mathrm{NH}$. The contribution of electric utilities to total emissions of these pollutants is lower than $1 \%$ of the total. ${ }^{38} \mathrm{CO}$ emissions have increased steadily since 1970 , both in absolute and relative amount. ${ }^{39}$ One explanation for the increase of $\mathrm{CO}$ emissions is that the adoption of $\mathrm{NO}_{x}$ control systems in natural gas boilers has reduced combustion efficiency, resulting in higher $\mathrm{CO}$ emissions. ${ }^{40}$ However, electric utilities still contribute only $1 \%$ of total CO emissions and CO has only limited and mostly uncertain 
effects on human health. ${ }^{\mathrm{n}} 4142$ Emissions of $\mathrm{VOC}$ and $\mathrm{NH}_{3}$ from electric utilities have remained roughly stable over time and are not currently regulated, as they account for only $0.2 \%$ and $0.6 \%$ of total emissions, respectively. ${ }^{43}$

Figure 2.4 illustrates annual trends in $\mathrm{SO}_{2}$ and $\mathrm{NO}_{\mathrm{x}}$ emissions by fuel type. From 2002 to 2013, $\mathrm{SO}_{2}$ emissions from coal declined dramatically as more coal plants installed flue gas desulfurization (FGD) systems. ${ }^{\circ}$ During the same time, $\mathrm{SO}_{2}$ emissions from petroleum have largely tracked the share of power sector generation from petroleum. Emissions of $\mathrm{NO}_{\mathrm{x}}$ have also declined as more coal plants installed selective catalytic reduction (SCR) controls to reduce emissions.

Figure 2.4. $\mathrm{SO}_{2}$ and $\mathrm{NO}_{x}$ Emission by Fuel, 2002-2013, in thousand metric tons. ${ }^{44}$

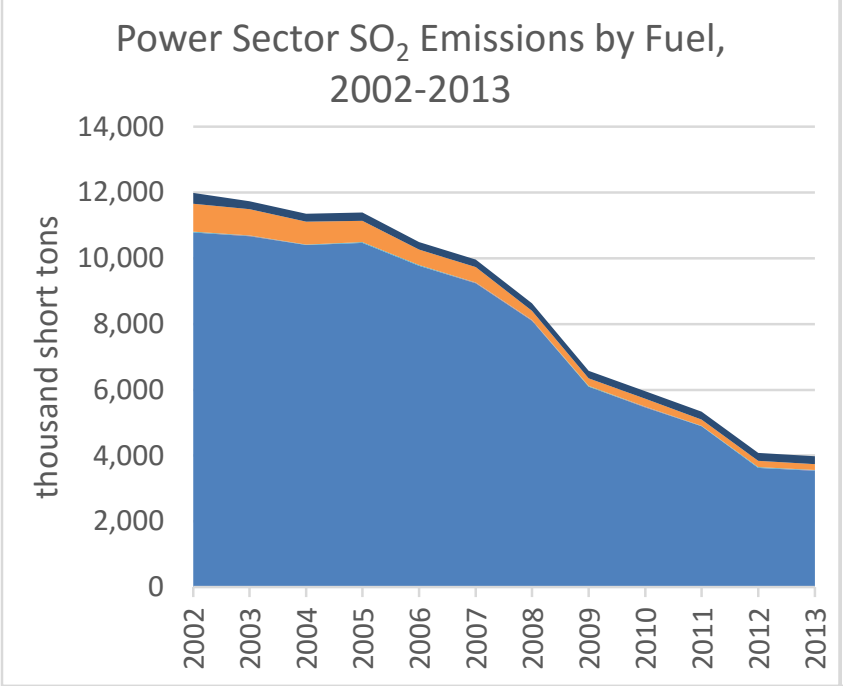

\section{Power Sector $\mathrm{NO}_{x}$ Emissions by}

Fuel, 2002-2013

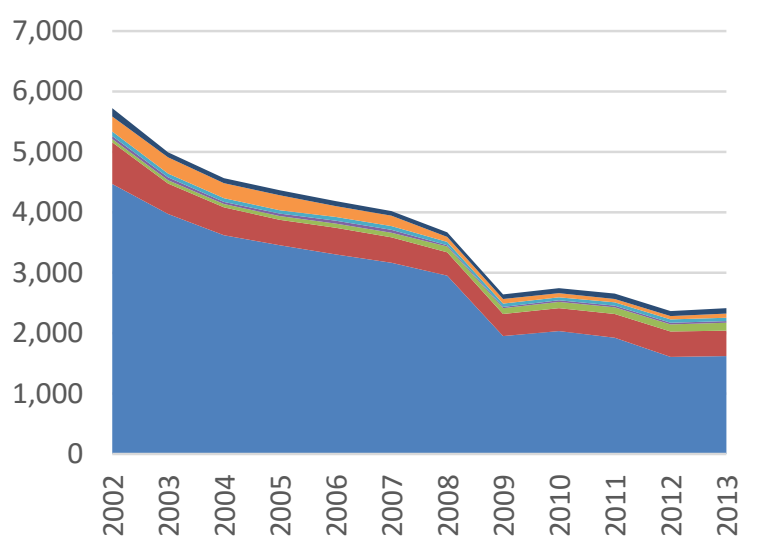

Coal

Coal-fired generation is responsible for the majority of $\mathrm{SO}_{2}$ and $\mathrm{NO}_{x}$ emissions from power generation, accounting for $89 \%$ of $\mathrm{SO}_{2}$ and $73 \%$ of $\mathrm{NO}_{x}$ emissions in 2013 . Natural gas combustion accounted for $17 \%$ of $\mathrm{NO}_{\mathrm{x}}$ emissions in 2013.

Notes: Other includes non-biogenic municipal solid waste, batteries, chemicals, hydrogen, pitch, purchased steam, sulfur, tire-derived fuels, and miscellaneous technologies. Other Biomass includes biogenic municipal solid waste, landfill gas, sludge waste, agricultural byproducts, other biomass solids, other biomass liquids, and other biomass gases (including digester gases and methane). Other Gases includes blast furnace gas, propane gas, and other manufactured and waste gases derived from fossil fuels.

Power generation also results in emissions of a number of hazardous air pollutants. In particular, power plants are the main sources of mercury emissions (50\%), acid gases (over

\footnotetext{
${ }^{\mathrm{n}}$ The Integrated Science Assessment for Carbon Monoxide by the EPA finds that the only likely causal relationship between carbon monoxide and human health impacts is observed for short-term cardiovascular morbidity. Evidence of other health effects is only suggestive, inadequate or not likely.

- See the Decomposition Analysis of $\mathrm{SO}_{2}$ Reductions for more details.
} 
$75 \%)$ and many toxic metals (20-60\%) in the United States. ${ }^{45}$ The power sector, and coal power generation in particular, is the largest source of mercury air emissions in the United States (Figure 2.5). Mercury emissions from the power sector declined by $57 \%$ between 2002 and 2011, while total emissions from other sources have declined by $86 \%$ (Figure 2.5).

\section{Figure 2.5. Annual Mercury Air Emissions from All Sources (Tons) ${ }^{46}$}

Tons of Mercury Emissions per Year in the United States

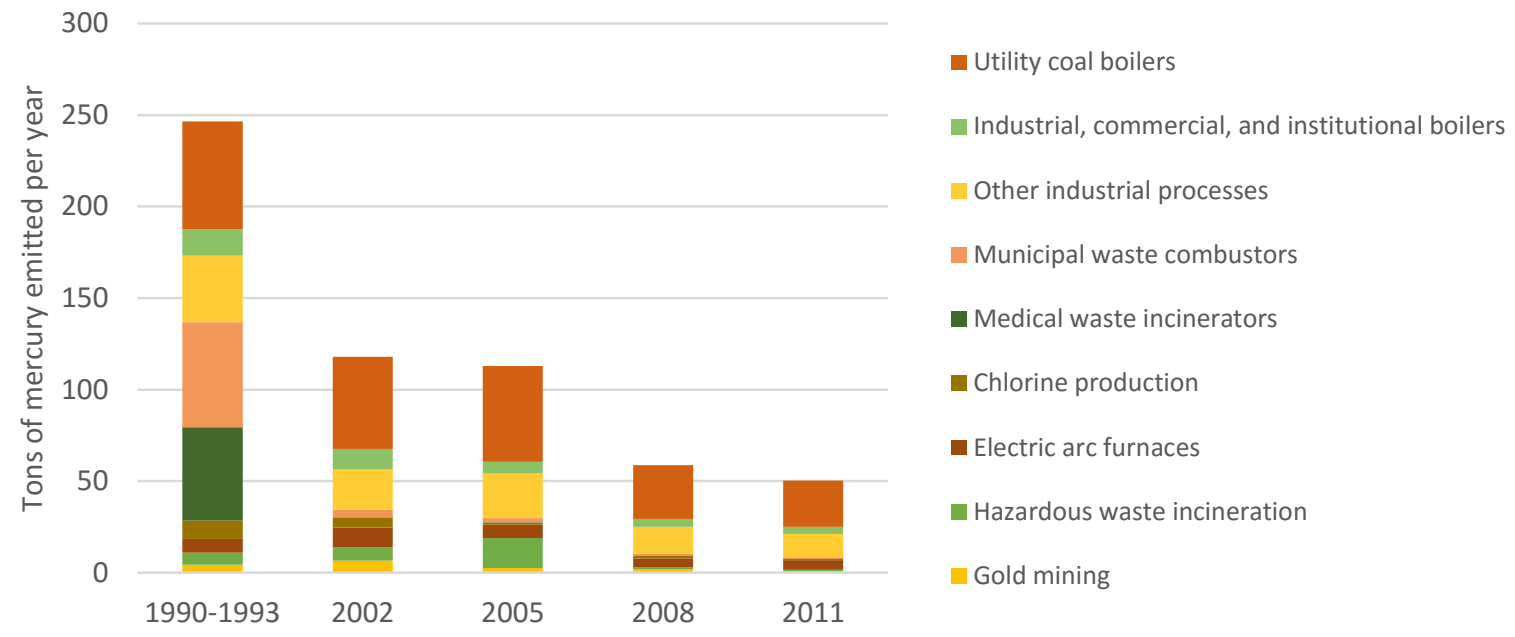

Power plants are the main sources of mercury emissions (50\%), acid gases (over $75 \%$ ), and many toxic metals $(20-60 \%)$ in the United States.

The observed decline in air pollution emissions is the outcome of regulation, technological trends (e.g., increased efficiency of power plants) and market conditions (e.g., low price of natural gas). Sections 2.2.1 and 2.2.2 address some of the underlying factors that have contributed to the observed reduction of air pollution from electricity generation.

\subsubsection{A Decomposition Analysis of Sulfur Dioxide Emission Reductions}

From 1994 to 2014, annual $\mathrm{SO}_{2}$ emissions from electric utilities have decreased by 11.7 million tons. The factors contributing to $\mathrm{SO}_{2}$ emission reductions have evolved over time, and the pace of emission reductions has been variable. Primary factors affecting $\mathrm{SO}_{2}$ emissions from coalfired power plants $^{p}$ include the sulfur content of coal, the heat content of coal, the heat rate of electricity generation, total generation from coal, and the fraction of coal-fired power plants using flue gas desulfurization (FGD) pollution controls.

\footnotetext{
${ }^{p}$ Coal-fired power plants are the predominant source of $\mathrm{SO}_{2}$ emissions from electric utilities (Figure 2.4) and have been responsible for more than 95 percent of $\mathrm{SO}_{2}$ emissions reductions since 1989. As such, this analysis focuses on changes in $\mathrm{SO}_{2}$ emissions from coal-fired power plants.
} 
The quality of coal-including the sulfur content and heat content of coal-varies by geographic region of coal production. In general, coal with a higher sulfur content leads to greater $\mathrm{SO}_{2}$ emissions than coal with a lower sulfur content. The heat content is the amount of thermal energy per unit of coal and is measured in British thermal units per short ton of coal (Btu/ton). Keeping all other factors fixed, a decline in the heat content of coal would result in greater consumption of coal (to produce the same amount of electricity) and greater subsequent $\mathrm{SO}_{2}$ emissions.

The thermal efficiency of electricity generation is measured by the heat rate, or the amount of thermal energy used to generate one kilowatt-hour of electricity, measured in British thermal units per kilowatt-hour (Btu/kWh). ${ }^{9}$ A generator with a lower heat rate can generate the same quantity of electricity while consuming less fuel, compared to a unit with higher heat rate. ${ }^{47}$ Heat rates depend in part on the type of equipment installed at a generating plant and can vary substantially across fuel and technology types. For example, in 2012 generators primarily powered by coal-fired boilers had heat rates ranging from 8,800 Btu/kWh to 25,000 Btu/kWh. ${ }^{48}$ A typical heat rate for a coal-fired power plant is around $10,400 \mathrm{Btu} / \mathrm{kWh}$.

Additional factors affecting $\mathrm{SO}_{2}$ emissions include the total amount of electricity generated from coal, and the percentage of coal-fired power plants using flue gas desulfurization (FGD) pollution controls. Figure 2.6 provides a graphical representation of these factors and their contribution to the change in $\mathrm{SO}_{2}$ emissions between 1994 and 2014.

\footnotetext{
q The heat rate is inversely proportional to the thermal efficiency of electricity generation. To express the efficiency of a generator as a percentage, divide the Btu content of a kilowatt-hour of electricity (which is 3,412 $\mathrm{Btu}$ ) by the heat rate. For example, the thermal efficiency of generator with a heat rate of $10,400 \mathrm{Btu} / \mathrm{kWh}$ is equal to $3,412 / 10,400=32.8 \%$.
} 
Figure 2.6. Factors Contributing to the Reduction in $\mathrm{SO}_{2}$ Emissions from Coal-Fired Power Plants: 1994-2014 5051

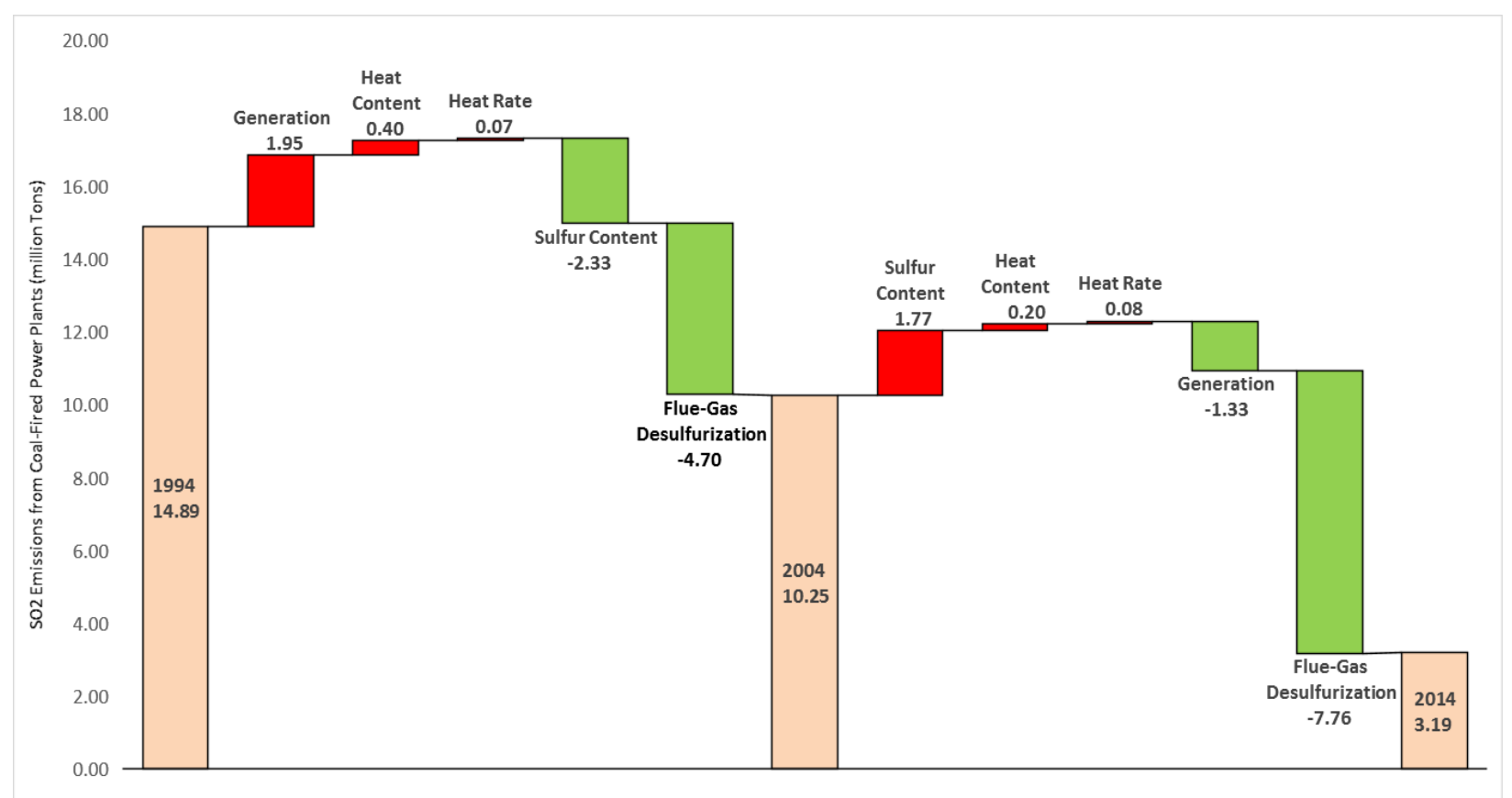

From 1994 to 2014, annual $\mathrm{SO}_{2}$ emissions from electric utilities have decreased by 11.7 million tons. Primary factors affecting $\mathrm{SO}_{2}$ emissions from coal-fired power plants include the sulfur content of coal, the heat content of coal, the heat rate of electricity generation, total generation from coal, and the fraction of coal-fired power plants using flue gas desulfurization (FGD) pollution controls.

Notes: The graph displays the contribution of different drivers to the trend of $\mathrm{SO}_{2}$ emissions. The vertical difference between the vertical bars that describe emissions in 1994, 2004 and 2014 is decomposed in different factors that affect emissions. The red bars indicate factors that have contributed to the increase of emissions and the green bars factors that have contributed to the decline of emissions, ceteris paribus. The contribution of each driver to the change of $\mathrm{SO}_{2}$ emissions is calculated assuming that all the other drivers remain constant during the time period being examined.

Between 1994 and 2004, increased coal power generation, lower heat content, and higher heat rates would (all other factors held constant) have contributed to increased $\mathrm{SO}_{2}$ emissions from the electric power sector. However, over the same time period, the average sulfur content of coal used in electricity generation declined, while the use of FGD scrubbers proliferated. The declining sulfur content and greater FGD use were the main drivers of reductions in $\mathrm{SO}_{2}$ emissions.

Between 2004 and 2014 different dynamics shaped the desulfurization trend. During this period, the heat content declined and the heat rate increased slightly, owing to greater coal production from the Illinois Basin. Additionally, the shift in coal production resulted in a higher average sulfur content. All other factors held constant, the higher sulfur content, lower heat content and higher heat rates would have led to greater $\mathrm{SO}_{2}$ emissions (Figure 2.6). However, 
these factors were offset by the decline in electricity generated from coal, and the continuing adoption of FGD scrubbers.

Appendix A provides a more thorough description of the trends in $\mathrm{SO}_{2}$ emissions, as well as the methodology used in this decomposition analysis.

\subsubsection{Major Pollutants from Electric Power Generation: Geographic Distribution}

Most emissions of CAPs originate in the eastern United States Figure 2.7. This is partly due to the distribution of population-power plants are located near demand centers in order to minimize transmission costs and losses-and in part due to the location of coal power generation, the largest source of air emissions.

Virtually all $\mathrm{SO}_{2}$ emissions from power generation are released by coal fired power plants-both with and without pollution controls - in the eastern United States (Figure 2.7, panel A). NOx and particulate matter emissions are more evenly distributed (Figure 2.7, panels B, C and D) as they are also released in significant quantities by natural gas power plants (Figure 2.1). 
Figure 2.7. Map of 2011 emissions from power plants of $\mathrm{SO}_{2}, \mathrm{NO}_{x}$ and Particulate Matter. ${ }^{52}$

Sulfur Dioxide

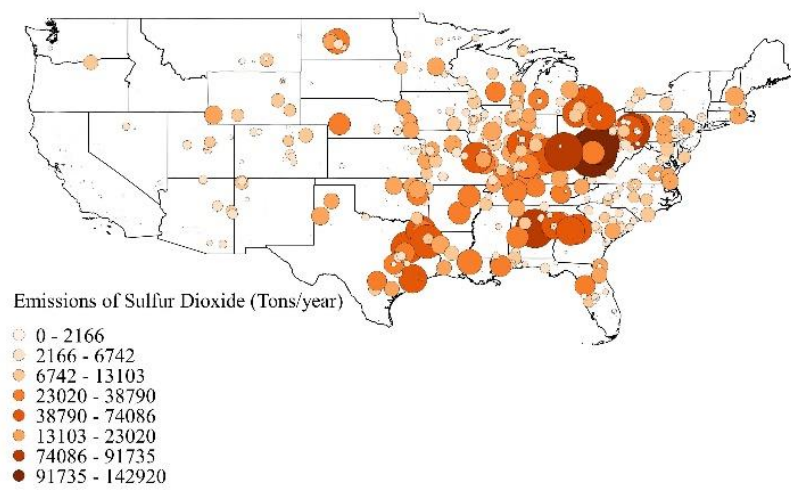

Nitrogen Oxides

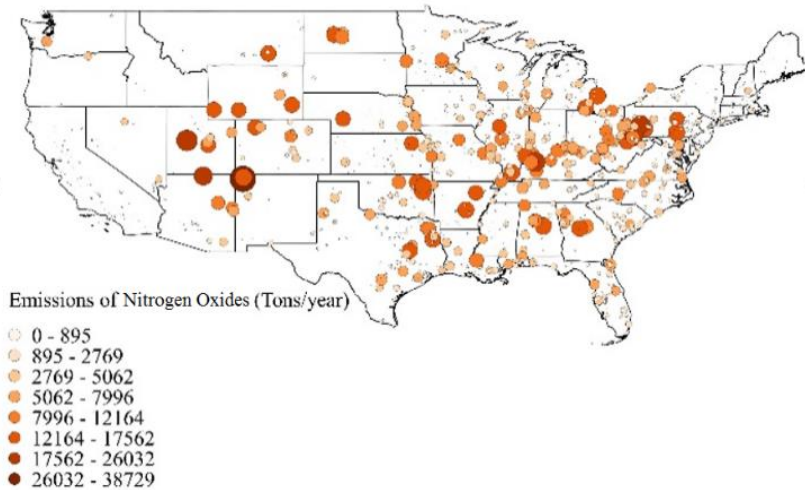

PM 2.5

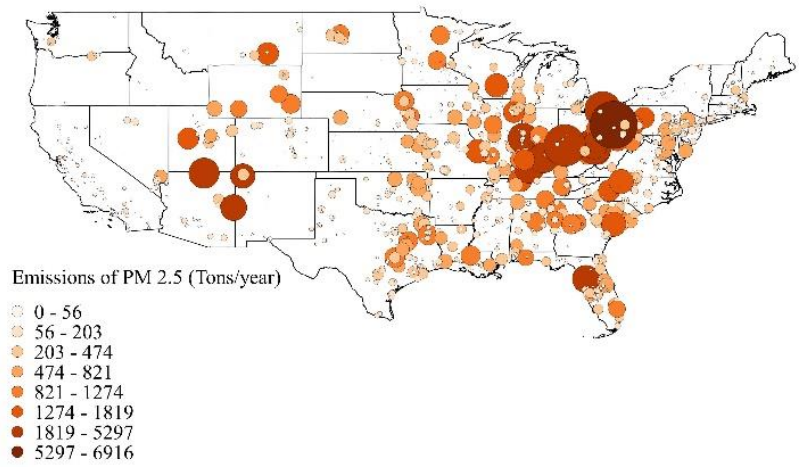

PM 10

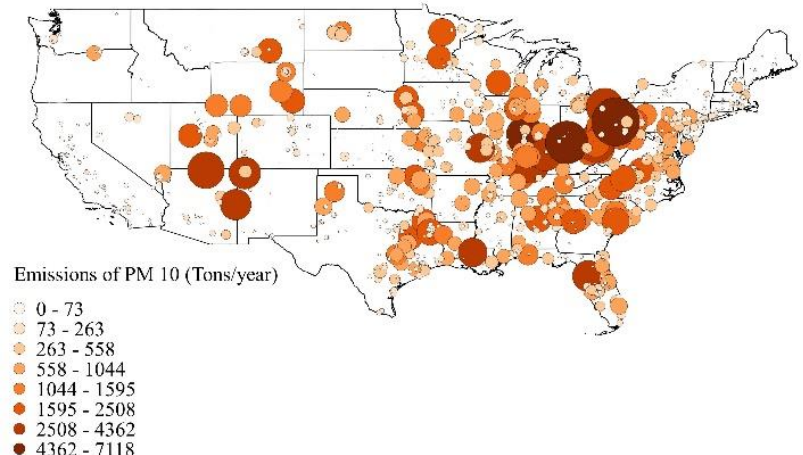

Virtually all $\mathrm{SO}_{2}$ is emitted from coal fired power plants—both with and without pollution controls-in the eastern United States. $\mathrm{NO}_{\mathrm{x}}$ and $\mathrm{PM}$ emissions are more evenly distributed, as they are also released in significant quantities by natural gas power plants.

Table 2.4 lists the top-emitting states for four major air pollutants from power generation. Ohio has the highest emissions for all four pollutants. Texas is the second largest emitter, mostly because of its size and the large number of plants located there. Pennsylvania, Indiana, and Kentucky also rank among the top emitting states. 
Table 2.4. Top-emitting states in 2014 of four CAPs from power generation (Thousands of Tons) $)^{53 \mathrm{r}}$

\begin{tabular}{rrrr}
\hline & $\mathbf{S O}_{\mathbf{2}}$ & & $\mathbf{N O}_{\mathbf{x}}$ \\
\hline Texas & 343.4 & Texas & 121.5 \\
Indiana & 290.7 & Pennsylvania & 119.0 \\
Ohio & 290.4 & Indiana & 108.6 \\
Pennsylvania & 262.2 & Kentucky & 86.9 \\
Kentucky & 202.0 & Ohio & 86.3 \\
\hline & $\mathbf{P M}_{10}$ & & $\mathbf{P M}_{\mathbf{2 . 5}}$ \\
\hline Ohio & 37.2 & Ohio & 33.8 \\
Texas & 19.6 & Texas & 12.5 \\
Pennsylvania & 16.2 & Pennsylvania & 11.5 \\
Indiana & 14.8 & Indiana & 10.0 \\
Kentucky & 13.9 & Florida & 9.6 \\
\hline
\end{tabular}

Air pollution emissions affect air quality in the immediate vicinity of power plants, but emissions can also travel long distances from the source. Local concentrations of air pollutants are driven by several factors, including the type of pollution, the location of pollution sources, atmospheric chemistry, weather patterns, geography and topography. Figure 2.8 illustrates the net result, which is that higher concentrations of $\mathrm{PM}_{2.5}$ in the atmosphere that are attributable to emissions from power plants are centered on the eastern United States, particularly the Ohio River Valley.

\footnotetext{
${ }^{r}$ The state-level $\mathrm{SO}_{2}$ and $\mathrm{NO}_{x}$ emissions data from the National Emissions Inventory have been updated through 2014 using data from EPA's Clean Air Markets Database. However, particulate emissions (PM10 and PM2.5) are presumed constant at 2011 levels and may be updated with the public release of the 2014 NEI v1, currently scheduled for August 15, 2016.
} 
Figure 2.8. Modeled Annual Mean $\mathrm{PM}_{2.5}$ Levels Attributable to Electricity Generating Units in 2016. 54

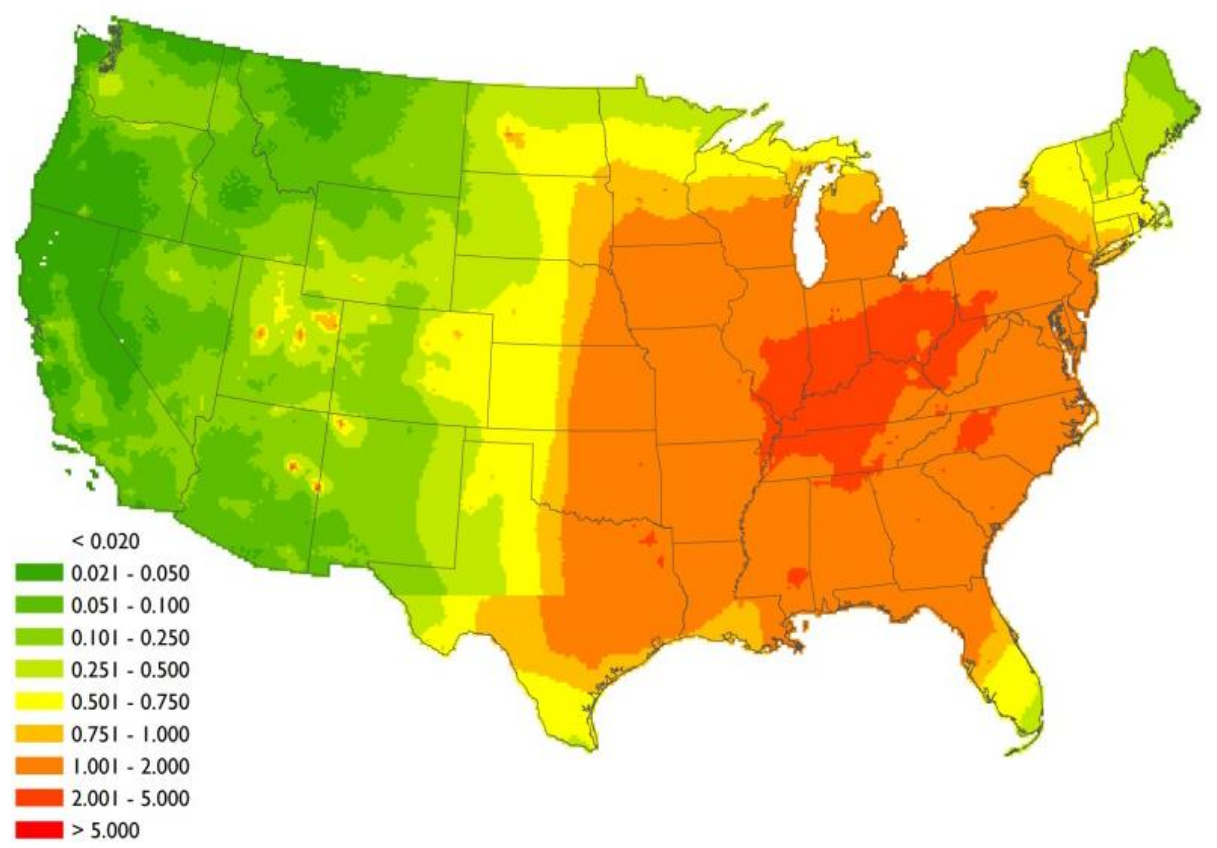

Particulate matter attributable to electricity generation is concentrated in the Midwest and Eastern half of the United States, especially in the Ohio River Basin.

Models of atmospheric circulation and chemistry that track air pollution have been developed to connect sources of pollution to receptors, ${ }^{s}$ and are discussed in greater detail in Appendix B. The distribution of air pollutants across geographic areas and ecosystems can be very complex. For example, airborne mercury can travel thousands of miles in the atmosphere, ${ }^{55}$ and some models estimate that significant amounts of mercury deposited ${ }^{t}$ in North America originated in other world regions. 565758

The atmospheric concentration of mercury is usually too low to have a measurable impact on human health or the environment. However, when mercury enters aquatic systems, some bacteria transform it into methylmercury $\left(\mathrm{CH}_{3} \mathrm{Hg}\right)$, which enters the aquatic food chain and accumulates in fish at concentration levels that are dangerous for human health.

Figure 2.9 displays the geospatial distribution of the wet deposition of airborne mercury, i.e., removal from the atmosphere by water. As shown on the map, in the United States, wet deposition of mercury concentrates along the Gulf Coast, lowlands and river basins of the Midwest, and the rainy slopes of the Pacific Northwest. As noted above, much of the mercury

\footnotetext{
${ }^{s} \mathrm{~A}$ receptor is the ecological or human entity exposed to a pollutant.
}

${ }^{\mathrm{t}}$ Includes both anthropogenic and natural sources. 
wet-deposited in the United States was not emitted locally. Similarly, mercury emitted by domestic sources also enters the global cycle and is deposited in other countries. ${ }^{4} 59$

Figure 2.9. Estimated geospatial distribution of the wet deposition of airborne mercury in the United States in $2014^{60}$

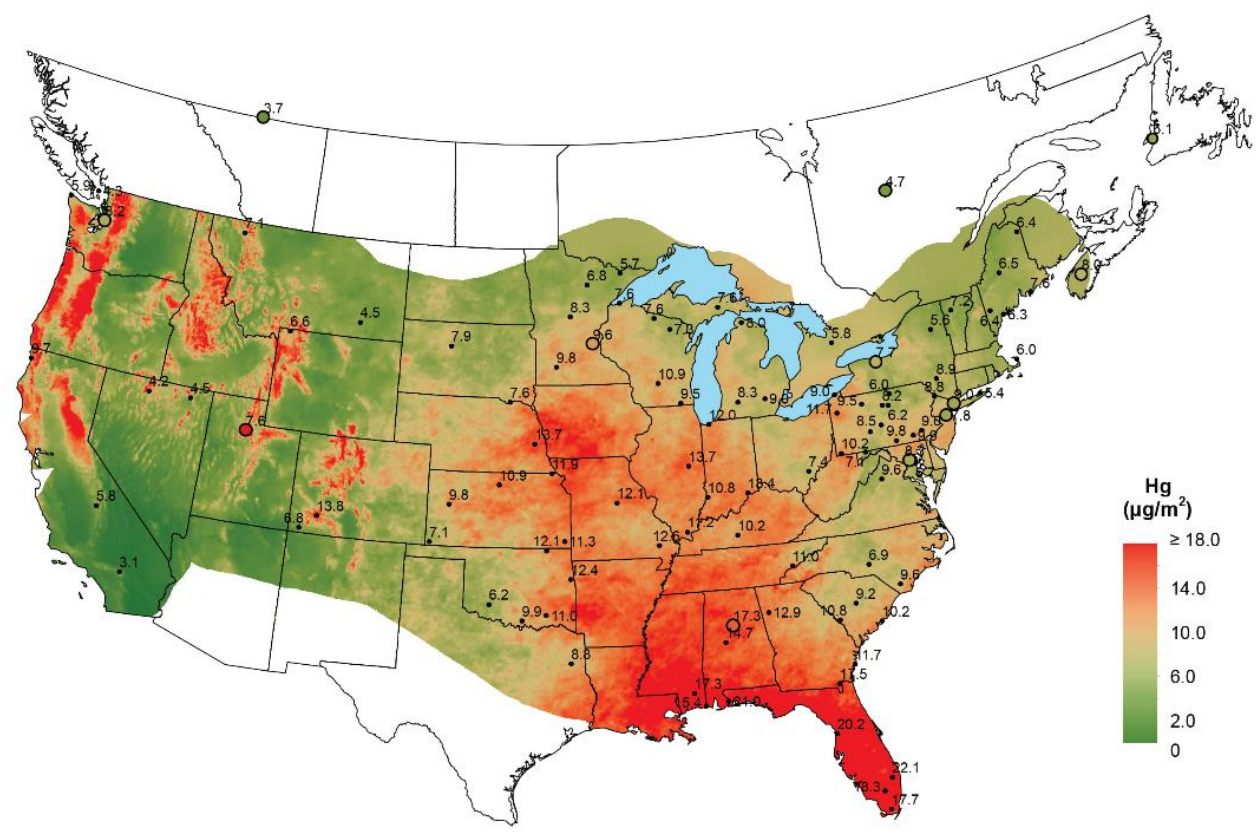

Wet deposition of airborne mercury is highest in Gulf States and along the Pacific coast. Mercury that enters the aquatic food chain is especially harmful to subsistence and recreational fishers.

Airborne emissions of mercury and other air toxics can also be deposited locally, and proximityto-the-source is commonly used as a surrogate for exposure to hazardous air pollutant emissions. For example, there are localized areas with elevated levels of mercury deposition around most U.S. power plants. ${ }^{61}$ Human and ecological health risks associated with mercury emission from power generation are greatest in regions most affected by locally deposited mercury. ${ }^{6263646566}$ A recent UNEP report finds that large point sources contribute up to threequarters of nearby deposition, and power plants contributes half of the deposition in the eastern part of the United States. ${ }^{67}$

\subsubsection{The Clean Air Act}

The basic structure of today's Clean Air Act (CAA) was established in 1970; major amendments were enacted in 1977 and in 1990. Table 2.6 provides a summary of the major sections and examples of how they relate specifically to the U.S. power sector.

\footnotetext{
"According to one model, North America is responsible for $5 \%$ of mercury deposited in Europe, $4 \%$ deposited in Asia, and $10 \%$ deposited in the Arctic.
} 
The 1990 amendments established the U.S. Acid Rain Program (ARP) and the world's first largescale cap-and-trade system aimed at reducing pollution. The program establishes an overall cap on $\mathrm{SO}_{2}$ emissions and creates allowances, or limited authorizations to emit, up to the level of the cap. Power plants comply with the program by holding enough allowances to cover their emissions. Under the program, covered facilities have the option to lower their emissions to free up allowances to trade or sell, or they may emit at levels higher than their allowance holdings and purchase additional allowances to cover the excess. Unused allowances can be banked for future use. ${ }^{68}$

The ARP is universally recognized as a success story in pollution regulation, with public health benefits that far exceeded costs and near-perfect compliance. ${ }^{69}$ Costs have been lower than expected, due to its compliance flexibility. For example, utilities were able to exploit unforeseen gains in generation efficiency and technical improvements that allowed burning low-sulfur coal at existing generators that were designed for high sulfur coal. ${ }^{70}$ Emissions trading has greatly reduced compliance costs by providing a flexible mechanism that allows for the most cost-effective emission reductions to be achieved first, using a market-based approach. Emissions trading also allows utilities to employ system-level compliance strategies, such as low emissions dispatching, whereby they may increase the utilization of low-emitting generating units (e.g., units retrofitted with an FGD) while decreasing the utilization of higher emitting generating units, as part of an strategy to achieve their overall emission targets at lower cost (Table 2.5)..$^{71} 72$ The trading of allowances is estimated to reduce compliance costs by more than $\$ 1$ billion annually compared to emission reduction systems that do not allow trading. ${ }^{73}$ However, a recent study by Chan et al. finds trade benefits to be less than one third of previous estimates, because allowance trading has resulted in a shift in emissions from lowdensity regions to regions with high population density, resulting in increased health risks compared to a no trade scenario. ${ }^{74}$ 
Table 2.5. Estimates of the cost of the ARP and of savings from emissions trading ${ }^{75}$

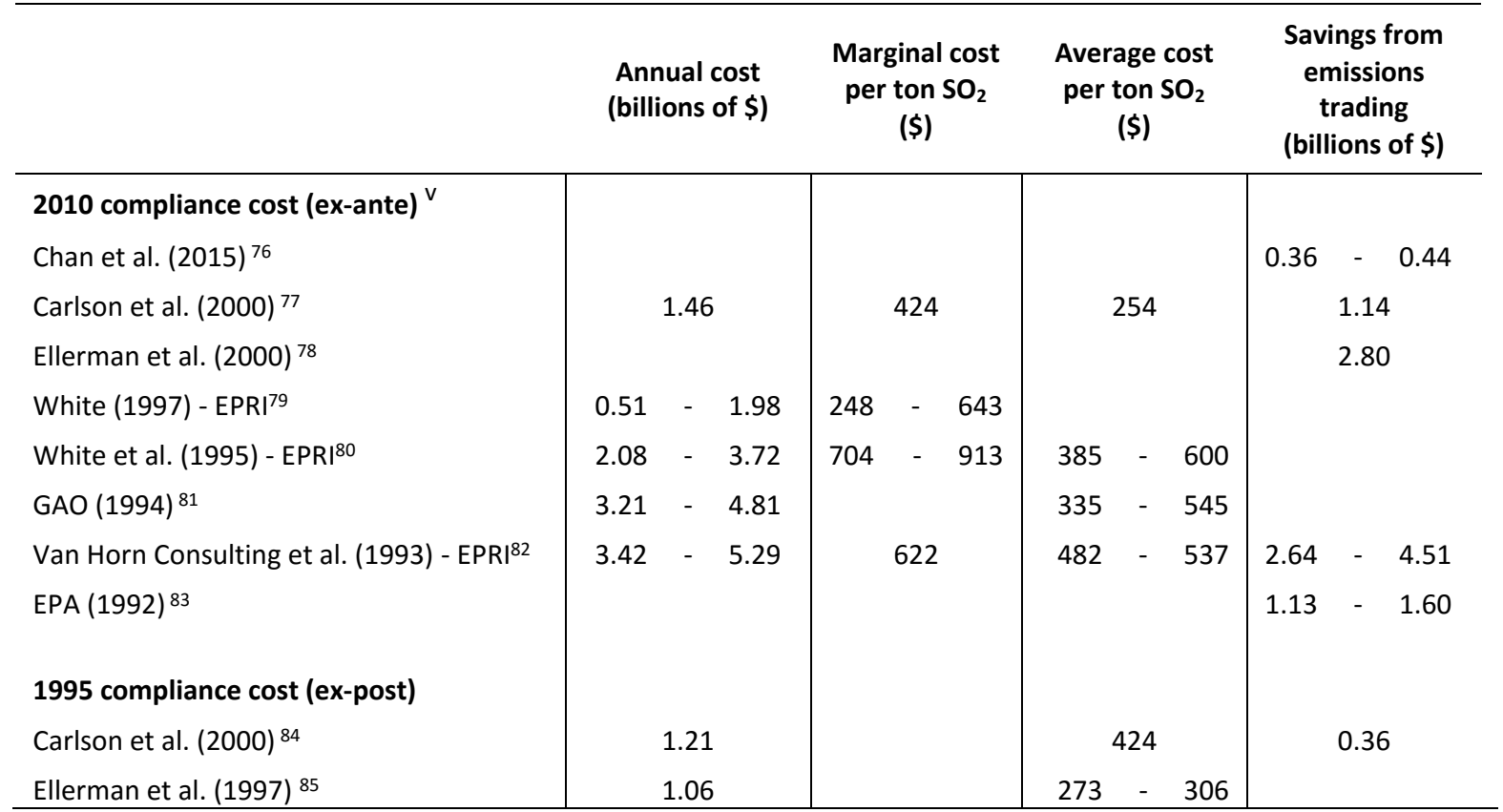

Notes: All dollar figures have been converted to $2015 \$$ to allow for comparison across studies.

Early analyses of the ARP overestimated the compliance costs by varying degrees, but almost all significantly underestimated the large public health benefits. For example, a 1990 independent study estimated a benefit-cost ratio that ranged between 0.5 and $4.5 .{ }^{86}$ Subsequent research has shown that the health and environmental benefits of the ARP are much larger and costs much smaller than originally estimated. ${ }^{87}$ The ARP program was conceived for protecting aquatic ecosystems, forests and agriculture from acidic depositions. ${ }^{88}$ However, as early as in 1990 the National Acid Precipitation Assessment Program (NAPAP) indicated that the environmental benefits from pollution reduction were relatively modest compared to health benefits. ${ }^{89} 90$ Estimates of health benefits vary but are considered to be around $\$ 50$ billion per year in 2010. ${ }^{91}$ This is a common finding across all the literature that assesses the physical and economic impact of air pollution from power generation: improved health conditions and lower risk of mortality are the dominant benefits of reducing power plant emissions. ${ }^{92}$ For this reason research efforts are mostly directed at estimating effects on health and mortality, as discussed in Section 2.4, and at determining their monetary value, as discussed in Section 2.5.

Emissions of mercury and other air toxics fall under a different provision of the Clean Air Act and are regulated through emission limits that are enforced at a facility level (see Table 2.6). The first federal regulation of mercury emissions from power plants-the Mercury and Air Toxics Standards (MATS) - was finalized in December 2011 and went into effect on April 16,

\footnotetext{
${ }^{\vee}$ Ex ante costs are based on forecasts or modelling rather than actual results. Ex-post costs are based on actual measurements.
} 
2012. It establishes emissions limits for new and existing coal- and oil-fired electricity generating units. ${ }^{93}$ MATS satisfies a statutory requirement that was triggered after EPA issued a finding that it was "appropriate and necessary" to regulate coal- and oil-fired electric utilities in December 2000. ${ }^{94}$

The MATS rule establishes emissions limits for new and existing coal- and oil-fired electricity generating units (EGUs) across several subcategories by fuel and technology type. The mercury emission limits range from 0.0002 to 0.040 pounds of mercury per gigawatt-hour of electricity (lb Hg/GWh) depending on the subcategory. ${ }^{95}$ Affected EGUs (with generating capacity greater than $25 \mathrm{MW}$ ) have three years from the date of rule's publication in the Federal Register to begin initial compliance (April 2015). Some units applied for and were granted an additional year (April 2016) to meet the emission limits. Based on EPA modeling of the rule, total mercury emissions from affected EGUs are projected to drop from 26.6 tons under a baseline scenario to 6.6 tons once the rule is fully implemented, a decline of $75 \%$ below the baseline scenario. ${ }^{96}$ The MATS rule also sets emission limits on acid gas HAP and non-mercury metallic HAP.

As a co-benefit of these HAP emission reductions, the emissions of $\mathrm{PM}_{2.5}$ and $\mathrm{PM}_{2.5}$ precursors including $\mathrm{SO}_{2}$ will also decline, leading to fewer $\mathrm{PM}_{2.5}$-related premature fatalities. Annualized total monetized benefits in 2016 are estimated between $\$ 37$ billion and $\$ 90$ billion, compared to projected annual compliance costs of $\$ 9.6$ billion. ${ }^{97}$ 
Table 2.6. Clean Air Act Provisions.

\begin{tabular}{l} 
Section and Description \\
\hline CAA Sections 109 and 110; NAAQS and SIPs \\
Several ubiquitous pollutants known as "criteria pollutants" are regulated under \\
Title I, section 109, which requires EPA to set National Ambient Air Quality \\
Standards (NAAQS) for certain pollutants, based on health studies. EPA is required \\
to periodically review the standards to ensure that they are based on current \\
science. \\
Meeting the NAAQS is state-federal partnership. States are responsible for \\
achieving and maintaining air quality that meets the NAAQS within their respective \\
jurisdictions. Section 110 of the CAA requires states to develop and submit to EPA \\
enforceable state implementation plans (SIPs), specifying how each state will attain \\
and maintain the federal air quality standards through regulations, permitting \\
programs, and other policies. Section 110 also requires state plans to prohibit \\
emissions that significantly contribute to nonattainment, or interfere with \\
maintenance, of air quality standards in other states. \\
For its part, EPA sets national emissions standards for vehicles and engines and new \\
stationary sources (see below), provides guidance and technical assistance to states, \\
and reviews state plans.
\end{tabular}

\author{
Trends and updates \\ Although levels of these pollutants \\ are substantially lower than in the \\ past, air pollution levels persistently \\ exceed the NAAQS for one or more \\ CAPs in many parts of the country. \\ Current NAAQS for ozone were \\ finalized in 2015. Current NAAQS for \\ PM were finalized in 2012.
}

To cut interstate pollution, EPA has issued the Cross-state Air Pollution Rule (and previously, the Clean Air Interstate Rule and $\mathrm{NO}_{\mathrm{x}}$ SIP Call Rule).

\begin{abstract}
CAA Section 111, New Source Performance Standards
Section 111 of the CAA requires EPA to set New Source Performance Standards (NSPS) for industrial categories that cause, or significantly contribute to, air pollution that may "endanger public health or welfare." NSPS are nationally uniform technology- based emissions standards for new and modified sources in industrial and other source categories. These nationally-uniform standards are set according to emission levels achieved by the best system of emission reduction (BSER) that has been adequately demonstrated, taking costs into consideration. Section 111 also requires state regulation of certain existing sources.
\end{abstract}

\section{CAA Section 112, NESHAP and MACT}

HAPs are regulated under Section 112 of the Clean Air Act, which sets National Emission Standards for Hazardous Air Pollutants (NESHAP). Congress established a list of nearly 190 hazardous compounds known or suspected to cause cancer or other serious health effects. Since the HAP sources are often specific industry processes or equipment types, EPA has identified categories of major industrial sources of these toxic air pollutants and developed emission standards for each, as required by the Act. These standards are based on the maximum reduction in emissions achievable (i.e., Maximum Achievable Control Technology, or MACT) taking into consideration costs and certain other factors, but must be at least as stringent as a floor level based on the emission performance of other similar sources.

\section{As a result of CAA regulations, power plants across the country have invested in pollution control equipment. Virtually all coal-fired units have electrostatic precipitators, baghouses, or other advanced controls for high levels of to remove particulate removal $\left(\mathrm{PM}_{10}\right.$ and $\mathrm{PM}_{2.5}$ ). ${ }^{\mathrm{w}}$ Additionally, a majority have installed pollution controls for $\mathrm{SO}_{2}$ and $\mathrm{NO}_{x}$ (Table 2.7). Some}

\footnotetext{
${ }^{w} \mathrm{~A}$ baghouse is an air pollution control device that removes particulates out of air or gas released from commercial processes or combustion for electricity generation. An electrostatic precipitator is a filtration device that removes fine particles, like dust and smoke, from a flue gas by electrostatically charging particles, which are then captured in electric filters.
} 
plants are also able to reduce $\mathrm{SO}_{2}$ emissions through the use of low-sulfur fuels or biomass cofiring.

Table 2.7. Pollution Control Technologies Installed in 2015.9899 100

\begin{tabular}{|c|c|c|}
\hline Technology & Description & $\begin{array}{l}\text { Installed on } \\
\% \text { Coal Units }\end{array}$ \\
\hline \multicolumn{3}{|l|}{$\mathrm{SO}_{2}$ Controls } \\
\hline FGD & $\begin{array}{l}\text { Post-combustion scrubbers that use a lime sorbent to remove } \mathrm{SO}_{2} \\
\text { from flue gas. New units can remove } 95 \%-98 \% \text { of } \mathrm{SO}_{2} \text {. }\end{array}$ & $60 \%$ \\
\hline FBC & $\begin{array}{l}\text { Fluidized bed combustion removes up to } 95 \% \text { of sulfur from coal } \\
\text { during combustion, preventing the formation of } \mathrm{SO}_{2} \text {. Low combustion } \\
\text { temperatures also reduce formation of } \mathrm{NO}_{x} \text {. }\end{array}$ & $8 \%$ \\
\hline IGCC & $\begin{array}{l}\text { Integrated gasification combined cycle plants use coal to produce a } \\
\text { syngas that is then combusted like natural gas. During gasification, } \\
\text { sulfur and particulates are removed from fuel, and syngas } \\
\text { combustion can be controlled to limit } \mathrm{NO}_{\mathrm{x}} \text { emissions. }\end{array}$ & $\begin{array}{c}3 \text { plants in the } \\
\text { U.S. }\end{array}$ \\
\hline \multicolumn{3}{|l|}{$\mathrm{NO}_{\mathrm{x}}$ Controls } \\
\hline SCR/SNCR & $\begin{array}{l}\text { Selective catalytic and selective non-catalytic reduction (SCR/SNCR) } \\
\text { post-combustion technologies remove } \mathrm{NO}_{x} \text { from flue gas, with } \mathrm{NO}_{\mathrm{x}} \\
\text { removal efficiencies of } 60 \%-70 \% \text { for } \mathrm{SNCR} \text { and better than } 80 \% \text { for } \\
\text { SCR. }\end{array}$ & $51 \%$ \\
\hline LNB & $\begin{array}{l}\text { Low- } \mathrm{NO}_{\mathrm{x}} \text { combustion technologies modify the combustion process to } \\
\text { limit } \mathrm{NO}_{\mathrm{x}} \text { formation, e.g., by regulating flame characteristics such as } \\
\text { fuel-air mixing. Removal efficiencies of } 40 \%-60 \% \text {. }\end{array}$ & $71 \%$ \\
\hline
\end{tabular}

Some plants use multiple pollution controls. For example, some plants use both fluidized bed combustion to reduce $\mathrm{NO}_{x}$ formation during combustion, in conjunction with SCR controls to remove $\mathrm{NO}_{x}$ from flue gas. As of $2015,59 \%$ of coal-fired units had both $\mathrm{SO}_{2}$ and $\mathrm{NO}_{x}$ controls; $32 \%$ had only $\mathrm{NO}_{x}$ controls; $1 \%$ had only $\mathrm{SO}_{2}$ controls, and $9 \%$ had no controls for either $\mathrm{SO}_{2}$ or $\mathrm{NO}_{\mathrm{x}}{ }^{101}$ 
Figure 2.10. 2015 Coal Power Plant Controls for $\mathrm{SO}_{2}$ and $\mathrm{NO}^{102}$

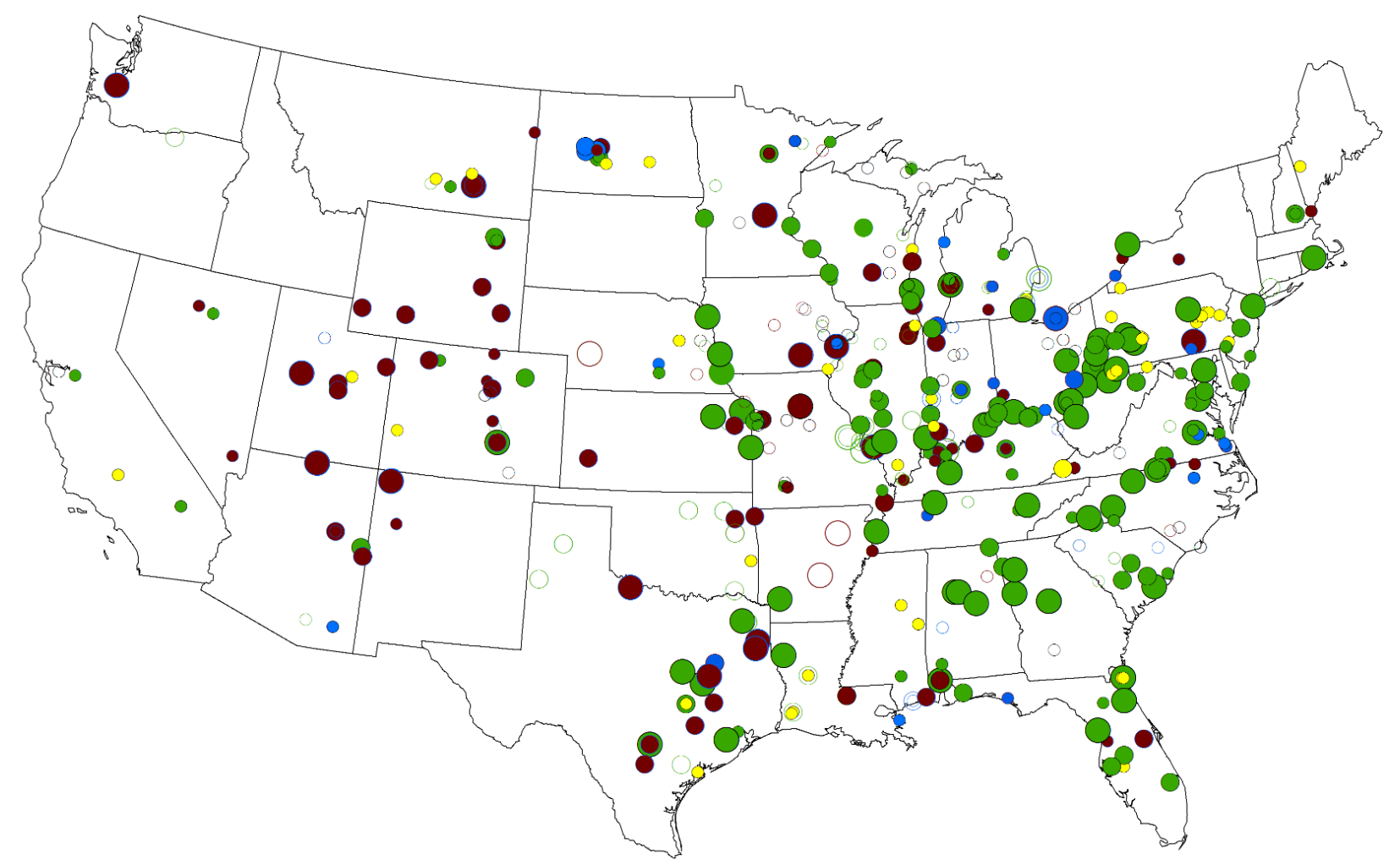

Scrubber and SCR/SNCR Scrubber

- Under $300 \mathrm{MW}$

- Under $300 \mathrm{MW}$

LNB and Under $1.0 \mathrm{lb} \mathrm{SO} / \mathrm{mmBtu}$ Under $1.0 \mathrm{lb} \mathrm{SO} / \mathrm{mmBtu}$

$300 \mathrm{MW}$ to $600 \mathrm{MW}$

Over $600 \mathrm{MW}$

SCR/SNCR

- Under $300 \mathrm{MW}$

- $300 \mathrm{MW}$ to $600 \mathrm{MW}$

Over $600 \mathrm{MW}$
- $300 \mathrm{MW}$ to $600 \mathrm{MW}$

Over $600 \mathrm{MW}$ FBC/IGCC

- Under $300 \mathrm{MW}$

$300 \mathrm{MW}$ to $600 \mathrm{MW}$

Over600 MW
Under $300 \mathrm{MW}$

$300 \mathrm{MW}$ to $600 \mathrm{MW}$

Over $600 \mathrm{MW}$

LNB

- Under $300 \mathrm{MW}$

$300 \mathrm{MW}$ to $600 \mathrm{MW}$

( Over $600 \mathrm{MW}$
- Under $300 \mathrm{MW}$

○ $300 \mathrm{MW}$ to $600 \mathrm{MW}$

over $600 \mathrm{MW}$

None

- Under $300 \mathrm{MW}$

○ $300 \mathrm{MW}$ to $600 \mathrm{MW}$

Over $600 \mathrm{MW}$

As of 2015, a majority of coal power plants used post-combustion pollution controls to remove pollutants from flue gas (solid green, dark red, or blue circles), or used fluidized bed combustion to prevent the formation of $\mathrm{SO}_{2}$. Most of the remaining plants used low NO x burners (hollow green or blue circles) and/or used low sulfur fuels (hollow green or dark red circles) to minimize emissions. Only a few plants had no pollution controls for $\mathrm{SO}_{2}$ or $\mathrm{NO}_{x}$ (hollow black circles).

\subsubsection{Technology and Innovation in Pollution Control}

Federal research and development programs - at both the U.S. Environmental Protection Agency (EPA) and the U.S. Department of Energy (DOE)-have led to significant advances in air pollution control technologies, including reductions in costs and improved environmental performance. In general, R\&D in pollution control technologies by itself is not expected to directly provide environmental benefits beyond regulatory requirements; rather, technological 
advancements provide lower-cost options to meet regulatory requirements and provide a technical basis for consideration of environmental regulations. This combination of regulatory drivers (e.g., the Clean Air Act) and technological improvements has worked to mitigate environmental impacts from electricity generation.

Emission controls for $\mathrm{PM}, \mathrm{SO}_{2}$, and $\mathrm{NO}_{x}$ have been commercially available since the $1970 \mathrm{~s}$. In particular, PM emission control devices using electrostatic precipitators and/or baghouse fabric filters are well established and have been adopted at nearly all large U.S. power plants. However, early technologies available for $\mathrm{SO}_{2}$ and $\mathrm{NO}_{x}$ emissions reductions had low collection efficiencies and could not be applied to all plant configurations. ${ }^{103}$ To improve and accelerate adoption of pollution controls, DOE established its Clean Coal Technology (CCT) program in 1979 to develop and demonstrate flue gas desulfurization (FGD) and $\mathrm{NO}_{\mathrm{x}}$ reduction technologies.

Retrospective analyses have found substantial benefits from DOE's CCT demonstration projects. For example, prior to the CCT program, $\mathrm{SO}_{2}$ scrubbers were costly to build and maintain, incurred substantial energy costs to run, and produced a sludge waste requiring considerable land use for proper disposal. Advancements from CCT demonstration projects include improved $\mathrm{SO}_{2}$ removal efficiencies of 95-98 percent; nearly 50 percent reductions in capital and operating costs; production of valuable byproducts such as wallboard-grade gypsum instead of waste; lower energy costs; and capture of multiple air pollutants. ${ }^{104}$

The CCT program also contributed to substantial advances in a portfolio of $\mathrm{NO}_{\mathrm{x}}$ control technologies. For example, low- $\mathrm{NO}_{x}$ burners (LNBs), overfire air, and reburning systems modify the combustion process to limit $\mathrm{NO}_{\mathrm{x}}$ formation during combustion. In addition, RD\&D programs supported U.S. adoption of post-combustion $\mathrm{NO}_{\mathrm{x}}$ removal technologies, including selective catalytic and non-catalytic reduction (SCR and SNCR) technologies.

Additional DOE programs have supported low-emission technologies including fluidized bed combustion (FBC) and integrated gasification combined cycle (IGCC). A summary of CCT technologies and their impacts is included in Table 2.8. 
Table 2.8. Impacts of Technology RD\&D in Pollution Control ${ }^{105} 106$

\begin{tabular}{ll}
\hline Technology & Impact \\
\hline Low nitrogen oxide burners & Now on $75 \%$ of U.S. coal power plants \\
& $\begin{array}{l}1 / 10 \text { to } 1 / 2 \text { the cost of older systems } \\
\$ 25 \text { billion national benefit }\end{array}$ \\
\hline Selective catalytic reduction & $\begin{array}{l}\text { Achieves } \mathrm{NO}_{\mathbf{x}} \text { reduction of } 80 \% \text { to } 90 \% \\
\text { Technology today costs half what it did in the } 1980 \text { s and is deployed } \\
\text { on about } 50 \% \text { of U.S. coal plants }\end{array}$ \\
\hline Flue gas desulfurization & Systems now cost $1 / 3$ what they did in the 1970 s \\
& $\begin{array}{l}\text { More than } 400 \text { commercial units deployed } \\
\text { Over } \$ 50 \text { billion in savings from lower FGD costs and environmental } \\
\text { improvement }\end{array}$ \\
\hline Fluidized bed combustion & $\begin{array}{l}170 \text { units deployed in the U.S. Inherently low NO } \text { emitting } \\
\text { technology capable of using coal waste fuels not previously usable. }\end{array}$ \\
& Providing economic/environmental benefits of $\$ 2$ billion through \\
& 2020 \\
\hline Integrated gasification & 7.5 GW projected to be operating in U.S. by 2020 . Estimated \\
combined cycle & economic/environmental benefits of over $\$ 12$ billion by 2020. \\
\hline
\end{tabular}

Another way to assess the potential for technological diffusion is to compare emissions of the most and least polluting coal power plants. Air Market Program data from 2015 reveals that the most polluting power plants have CAP emissions per unit of electricity many times larger than the least polluting power plants (Table 2.9). The median coal power plant still has CAP emissions 10 times larger than power plants at the fifth percentile (Table 2.9).

Table 2.9. Distribution of Pounds of Criteria Emissions per MWh by Coal Power Plants, 2015. ${ }^{107}$

\begin{tabular}{lccccccc}
\hline & Mean & $\begin{array}{l}\text { Standard } \\
\text { deviation }\end{array}$ & $\begin{array}{l}\text { 5th } \\
\text { percentile }\end{array}$ & $\begin{array}{l}\text { 25th } \\
\text { percentile }\end{array}$ & $\begin{array}{l}\text { 50th } \\
\text { percentile }\end{array}$ & $\begin{array}{l}\text { 75th } \\
\text { percentile }\end{array}$ & $\begin{array}{l}\text { 95th } \\
\text { percentile }\end{array}$ \\
\hline $\mathrm{SO}_{2}$ & 4.2 & 6.2 & 0.2 & 0.9 & 2.2 & 4.9 & 15.8 \\
$\mathrm{NO}_{x}$ & 2.0 & 1.3 & 0.5 & 0.9 & 1.7 & 2.7 & 4.3 \\
\hline
\end{tabular}

\subsection{PROJECTIONS OF AIR EMISSIONS FROM ELECTRICITY GENERATION}

Future trends of emissions depend on many factors, including the fuel mix, technological progress, policy and energy demand. The EPSA Base Case models the energy sector out to 2040 using EPSA-NEMS, an integrated energy system model. ${ }^{\mathrm{x}}$ The EPSA Base Case input assumptions

\footnotetext{
${ }^{x}$ The version of the National Energy Modeling System (NEMS) used in this report has been run by OnLocation, Inc., with input assumptions determined by DOE's Office of Energy Policy and Systems Analysis (EPSA). Since this analysis was commissioned by EPSA and uses a version of NEMS that differs from the one used by the U.S. Energy Information Administration (EIA), the model is referred to as EPSA-NEMS.
} 
were based mainly on the final release of the 2015 Annual Energy Outlook (AEO 2015), with a few exceptions as noted below, and then updated to include the Clean Power Plan and tax extenders. ${ }^{y}$ As with the AEO, the ESPA Base Case provides one possible scenario of base case energy sector demand, generation, and emissions from present day to 2040, and it does not include future policies that might be passed or future technological progress. The EPSA Base Case input assumptions were based mainly on the final release of the 2015 Annual Energy Outlook (AEO 2015), with a few updates that reflect current technology cost and performance estimates, policies and measures. Assumptions from the EIA 2015 High Oil and Gas Resources Case were used; it has lower gas prices similar to those in AEO 2016.

The EPSA Base Case scenario assumes that electricity demand will grow at about $1 \%$ per year from 2015 to 2040, in line with recent trends. ${ }^{108}$ Natural gas power generation is expected to increase due to low natural gas prices, while coal generation is expected to decline (Figure 2.11). Nuclear power is constant while renewable electricity generation more than doubles (Figure 2.11). Emissions of $\mathrm{SO}_{2}$ and of mercury are expected to sharply decline in 2016 as full compliance begins with the MATS rule. ${ }^{2}$ After 2016, emissions of $\mathrm{SO}_{2}$ and mercury continue to decline, but at a much slower pace, about $1.9 \%\left(\mathrm{SO}_{2}\right)$ and $2.0 \%(\mathrm{Hg})$ per year from 2016 until 2040). ${ }^{109} \mathrm{NO}_{\mathrm{x}}$ emissions decline at a rate of about $1.7 \%$ per year from 2015 until 2040). ${ }^{110}$

\footnotetext{
${ }^{y}$ The EPSA Base Case achieves the broad emission reductions required by the Clean Power Plan. While states will ultimately decide how to comply with the Clean Power Plan, the EPSA Base Case assumes that states choose the mass-based state goal approach with new source complement and assumes national emission trading among the states, but does not model the Clean Energy Incentive Program because it is not yet finalized. The EPSA Base Case also includes the tax credit extensions for solar and wind passed in December 2015. In addition, the utility-scale solar and wind renewable cost and performance estimates have been updated to be consistent with EIA's AEO 2016. Carbon capture and storage (CCS) cost and performance estimates have also been updated to be consistent with the latest published information from the National Energy Technologies Laboratory.

${ }^{\mathrm{z}}$ Initial compliance with MATS began in April 2015. Some plants received a 1-year extension and came into compliance in April 2016.
} 
Figure 2.11. Projected Net Electricity Generation by Fuel, 2015-2040. ${ }^{111}$

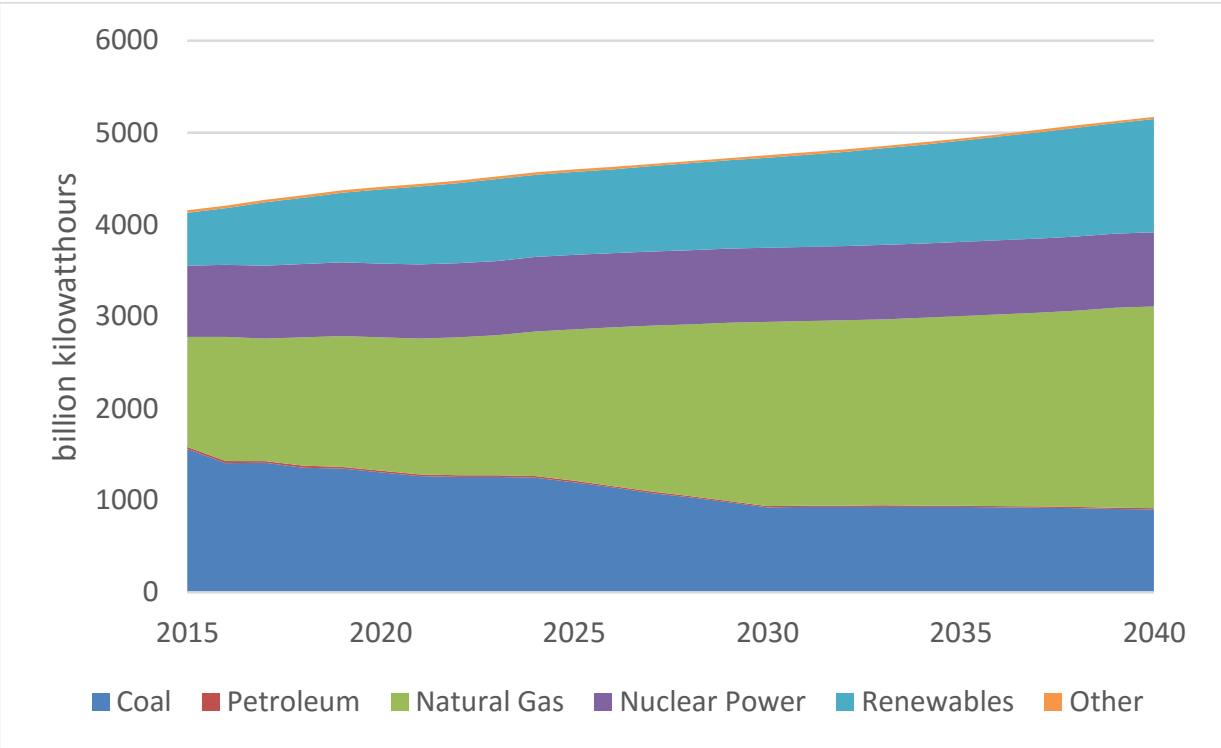

Projections of electricity generation through 2040 under current market trends and energy policies. The largest growth comes from renewables, including wind and solar. Generation from fossil fuels declines slightly, while nuclear power remains roughly constant.

Notes: Renewables includes conventional hydroelectric, geothermal, wood, wood waste, biogenic municipal waste, landfill gas, other biomass, solar, and wind power.

Figure 2.12. Historic and Projected $\mathrm{SO}_{2}, \mathrm{NO}_{x}$ and mercury air emissions from electricity generation: 1990-2040. 112113114

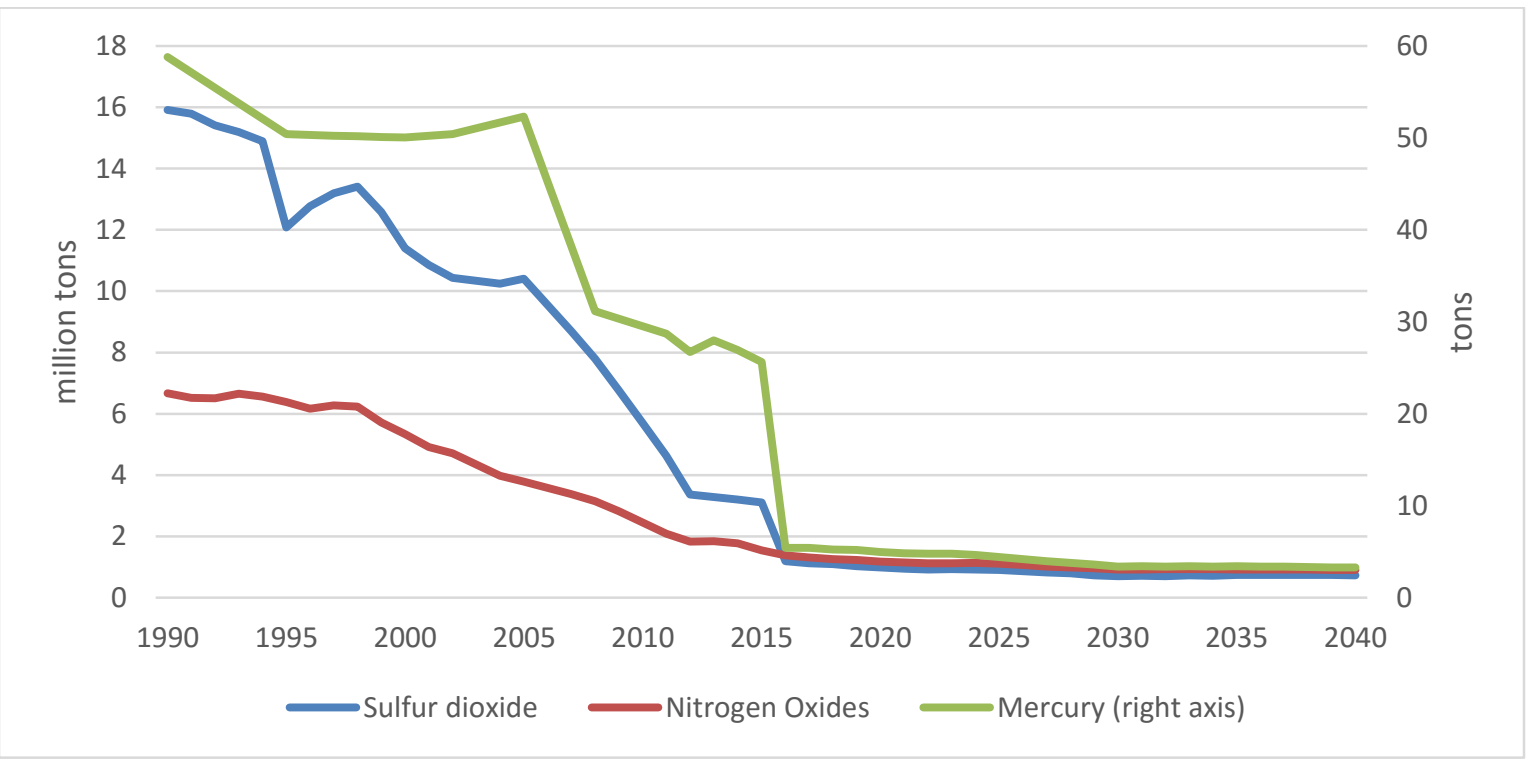

Sulfur dioxide and mercury emissions are projected ${ }^{\text {aa }}$ to drop from 2015 to 2016 as affected coal units come into compliance with the MATS rule. After 2016, emissions largely track coal-fired generation.

aa Historical data are shown for the years 1990 through 2014. Values for 2015 through 2040 are projections. 


\subsection{PHYSICAL IMPACTS OF AIR POLLUTION FROM POWER GENERATION}

While many studies estimate the impacts of single pollutants at specific locations, literature that tracks the effects of pollution specifically from power generation is limited. The main difficulty is the attribution of pollution damages to one particular sector. In order to connect observed impacts to power generation emissions specifically, researchers must use models that track pollution from sources to receptors. This is a complex task that explains why few studies are available. Appendix $B$ describes the models used in the literature in order to provide better insights on how to interpret air pollution impact estimates. These models typically, but not always, use three steps to quantify the impact of pollution.

The first step is to determine the geographic distribution of ambient concentrations of pollution. This is usually done using atmospheric circulation and chemistry models that track how pollution moves in the atmosphere and how primary pollution gets transformed into secondary pollution. The Community Multi-scale Air Quality (CMAQ) modeling system developed by EPA is widely used to study air quality. ${ }^{115}$ The model tracks the atmospheric distribution and deposition of pollution with a high geographical and temporal resolution. The model accounts for the effect of winds and other meteorological variables, geography and atmospheric chemistry in determining the distribution and chemical transformation of primary pollutants into secondary pollutants. The output of the model is finally adjusted to reflect actual observations from monitoring stations.

The second step estimates how many people and man-made or natural assets are exposed to pollution. Exposure is defined as the contact between a target receptor and a pollutant at the outer boundary of the receptor. ${ }^{116}$ Exposure gives rise to a hazard that may cause damage. Precise estimates of pollution impacts require a detailed characterization of sensitive assets and affected populations. For example, the environmental Benefits Mapping and Analysis Program - Community Edition (BenMAP-CE) model used by the EPA includes hundreds of health impact functions that quantify air pollution health impacts among populations affected by poor air quality exposure categories. ${ }^{117}$

The third and final step usually quantifies pollution impacts to estimate the actual damage caused by pollution. After determining ambient air pollution concentration and exposure, the impact studies use impact functions from the literature to estimate the actual damage caused by pollution. 
Table 2.10. Summary of physical impacts of the most common pollutants. ${ }^{118} 119120121$

\begin{tabular}{|c|c|c|c|c|c|}
\hline & Human Health & $\begin{array}{l}\text { Crops and } \\
\text { Timber }\end{array}$ & Materials & Visibility & Recreation \\
\hline \multirow[t]{2}{*}{$\mathrm{NO}_{\mathrm{x}}$} & COPD & & Material & & eutrophication \\
\hline & IHD & & deterioration & & \\
\hline \multirow[t]{2}{*}{$\mathrm{SO}_{2}$} & Asthma & $\begin{array}{l}\text { Damages to } \\
\text { forests }\end{array}$ & $\begin{array}{l}\text { Material } \\
\text { depreciation }\end{array}$ & & $\begin{array}{l}\text { Damages to } \\
\text { forests }\end{array}$ \\
\hline & Cardiac & & & & \\
\hline \multirow[t]{4}{*}{$\mathrm{O}_{3}$} & Chronic asthma & & $\begin{array}{l}\text { Rubber } \\
\text { deterioration }\end{array}$ & & $\begin{array}{l}\text { Damages to } \\
\text { forests and }\end{array}$ \\
\hline & Acute-exposure mortality & Timber loss & & & wilderness \\
\hline & Respiratory problems & & & & areas \\
\hline & Acute asthma attacks & & & & \\
\hline \multirow[t]{4}{*}{$\mathrm{PM}_{2.5}$} & Premature death & & & Loss of visibility & \\
\hline & Nonfatal heart attacks & & & & \\
\hline & Hospital admissions & & & & \\
\hline & $\begin{array}{l}\text { ER visits for asthma, acute } \\
\text { bronchitis, upper and lower } \\
\text { respiratory symptoms }\end{array}$ & & & & \\
\hline $\mathrm{PM}_{10-2.5^{\mathrm{bb}}}$ & Chronic bronchitis & & & & \\
\hline
\end{tabular}

Notes: COPD: chronic obstructive pulmonary disease; IHD: ischemic heart disease

Pollutants emitted by the electric power sector cause damage to human health (increased morbidity and mortality), to crops and timber production (productivity losses), and to materials (deterioration and increased maintenance costs). They reduce visibility and harm ecosystems, with losses to recreational value and ecological services. Table 2.10 lists the major impacts, by sector, of criteria air pollutants.

Health impacts constitute the largest fraction of economic damages of air pollution. In order of to be comprehensive, estimated health impacts include reduced organ functionality; increased asthma attacks; doctor visits, school and work absences; emergency room visits, hospital admission and heart attacks; and premature death. Emissions of coarse particulate matter (PM10-2.5 - i.e., particulate matter that is between 10 and $2.5 \mu \mathrm{m}$ in diameter) cause chronic obstructive pulmonary disease, asthma, and hospital respiratory and cardio-vascular admissions but have not been associated with increased mortality. ${ }^{122}$ However, fine particles $\left(\mathrm{PM}_{2.5}\right)$ are more harmful because they translocate from the lungs to blood and accumulate in other parts of the body, increasing short- and long-term mortality and morbidity. ${ }^{123}$

Human exposure to ground-level ozone reduces lung function, generates inflammation of the airways, and causes symptoms such as chest pain, coughing, wheezing and shortness of breath, even for people with no pre-existing respiratory ailments. ${ }^{124} 125$

${ }^{\mathrm{bb}} \mathrm{PM}_{10-2.5}$ is coarse particulate matter with diameter between 10 and $2.5 \mu \mathrm{m}$. 
The health impacts are estimated by comparing mortality under a reference scenario and a scenario in which pollution is changed. The marginal impact of pollution estimates the change in impacts resulting from increasing the level of pollution by one unit only. The average impact of pollution is determined by dividing the total health impacts by the total level of pollution. $A$ concentration-response (or dose-response) function is used to study how the health effects change when the concentration of pollutants changes. The concentration-response function is derived from toxicological studies, human clinical trials and observational epidemiology studies. ${ }^{126}$ In some cases expert elicitation is used to reduce uncertainty about the relationship between pollutants and health. ${ }^{127}$

Ground-level ozone concentrations and sulfur dioxide have been shown to reduce agricultural and timber productivity. ${ }^{128} 129130131$ In an assessment of the costs and benefits of the Clean Air Act, the EPA has used the Forest and Agricultural Sector Optimization Model (FASOM) to study the impact of reductions in tropospheric ozone concentrations on the productivity of crops and timber. ${ }^{132} 133$

Sulfur dioxide, nitrogen oxides and hydrogen ions are the main precursors to acid rain, which reduces the structural integrity and aesthetic appearance of building materials. ${ }^{134}$ Acidic deposition degrades metals and accelerates the erosion of marble and limestone. Doseresponse functions from the literature are used to estimate the loss of materials due to pollution. By applying the loss coefficients to inventories of carbonate stone, galvanized steel, carbon steel, and painted wood surfaces, it is possible to calculate the additional maintenance costs caused by acidic deposition. ${ }^{135}$

Air pollution reduces visibility, with widespread negative effects, including impacts on visibility in natural parks, reduced road and air safety, amenity values, and other non-use values resulting from the existence of pristine environmental locations that are free of pollutioninduced haze. ${ }^{136} \mathrm{~A}$ recent cost-benefit analysis of Clean Air Act programs provides a list of studies that are used to estimate the concentration-response function for visibility impact estimates of air pollution. ${ }^{137}$

Pollution has direct and indirect impacts on both aquatic and terrestrial ecosystems because air pollution accumulates in soils and waters. The pollutants may cause ecological changes, such as long-term acidification of soils or surface waters, soil nutrient imbalances affecting plant growth, and loss of biodiversity. ${ }^{138}$ In the United States, the National Trend Network provides data on precipitation chemistry that allow calculating and mapping deposition of pollutants over the whole country. Damages to ecosystems are calculated using information on "critical loads"-i.e., the threshold of air pollution deposition below which specified harmful ecological effects do not occur - and are prepared by the National Atmospheric Deposition Program. ${ }^{139} 140$ 
There is growing concern about the harmful effects of emissions of heavy metals-mercury $(\mathrm{Mg})$, arsenic $(\mathrm{As})$, chromium ( $\mathrm{Cr}$ ), and nickel (Ni)-from power plants. These metals are also known as air toxics and likely cause cancer and pose other serious health risks.

Coal combustion releases mercury into the air. In general, in the United States the concentration of mercury in ambient air is too low to represent any risk to human health. ${ }^{141}$ However, mercury that is deposited in aquatic systems is transformed by bacteria into methylmercury $\left(\mathrm{CH}_{3} \mathrm{Hg}\right)$, which readily accumulates in animal tissues and produces several harmful effects. Sufficient accumulations of methylmercury can kill an organism. Direct or indirect consumption of fish is the major source of exposure to $\mathrm{CH}_{3} \mathrm{Hg}$ for humans. $\mathrm{CH}_{3} \mathrm{Hg}$ is easily absorbed by the human body, and it distributes over all tissues, harming nearly all vital organs, including the brain. $\mathrm{CH}_{3} \mathrm{Hg}$ easily passes through the placenta, and fetuses are most sensitive to the adverse impact of $\mathrm{CH}_{3} \mathrm{Hg}$. Prenatal exposure to chronic low doses of methylmercury is associated with cognitive deficits. ${ }^{142} 143$ Dose-response functions estimated in the literature indicate that there is a loss of 0.020 IQ points in the newborn when the mother absorbs more than $6.7 \mu \mathrm{g} /$ day. In order to prevent serious consequences to newborns, national health agencies recommend that pregnant women limit consumption of fish that are at the top of the aquatic food chain, such as tuna or swordfish, because they have the highest concentrations of $\mathrm{CH}_{3} \mathrm{Hg}$ in their tissues. In adults there is evidence linking mercury exposure to adult cardiovascular, immune and reductive system effects but the evidence of impacts for adults at levels of exposure commonly seen in the United States is less well established. ${ }^{144}$

\subsection{ECONOMIC EVALUATION OF IMPACTS}

The physical impacts of air pollution can be expressed in monetary terms, with the immediate advantage of allowing a quantitative assessment of air pollution impacts on many sectors and assets. $^{145}$

Monetary evaluations of impacts are used to quantify the economic benefits of air quality policies. ${ }^{c c}$ The Executive Order 12866 requires federal agencies to estimate the benefits of regulation that, among other criteria, have an annual impact on the economy greater than $\$ 100$ million. ${ }^{146}$ The EPA routinely performs economic assessments of environmental regulation and these are used together with other information to guide policy decisions, depending on the statutory goals and requirements of each regulatory action.

Economic estimates of pollution costs are subject to uncertainty for several reasons (Appendix B). Many goods and services that are affected by air pollution are not traded in markets and

\footnotetext{
${ }^{c c}$ Note that the economic cost of pollution is equivalent to the economic benefit of pollution reduction.
} 
thus their monetary value is not readily available. For example, one of the main adverse impacts of air pollution is the increased risk of mortality. Estimating the monetary cost of increasing the risk of mortality is a complex exercise subject to a many uncertainties. Impacts on ecosystems are also challenging to quantify in economic terms.

Notwithstanding these limitations, there is a growing literature that uses state-of-the art modelling and evaluation tools to provide economic estimates of air pollution costs. There are large differences in the literature, and these estimates are subject to change over time as new evidence and more accurate methods become available. However, a key finding across all studies is quite consistent: the economic benefits of reducing air pollution-particularly fine particles, ozone, and their precursors - range from billions to hundreds of billions of dollars each year.

The next section reviews some methodological issues and Section 2.5.1 presents a summary of cost estimates of air pollution from power generation.

\subsubsection{Methodological Considerations}

\section{Average and marginal cost of pollution:}

The literature generally presents either average or marginal costs of air pollution. The average cost of air pollution is determined by calculating the total cost of air pollution and dividing it by total emissions. The marginal cost of pollution is more complex to determine as it requires estimating the impact of the last (the marginal) unit of pollution.

If the marginal cost is constant at different levels of pollution, the two measurements are equivalent. But if marginal costs increase as pollution increases, as is often the case, the estimate of the marginal benefit of pollution reduction is higher than the average benefit. This is an important distinction among different studies. Muller and Mendelsohn is an example of a study that estimates the marginal benefit of emissions reduction. ${ }^{147}$

Estimates of marginal costs of pollution provide some advantages over estimates of average pollution cost. ${ }^{148}$ First, to the extent that the marginal benefit of pollution reduction can be determined with a reasonable degree of accuracy, it is theoretically possible to use this information to determine the economically efficient level of pollution. ${ }^{\text {dd }}$ Second, the marginal

\footnotetext{
dd Assuming that the monetary benefits of pollution reduction is known, this argument considers that at the optimal level of pollution, the marginal private cost and the marginal social benefit of pollution reduction must be equal. Emitting one unit of pollution below this socially optimal level implies that the cost of reducing one unit of pollution is lower than the benefit. This is not an economically efficient level of emissions. Emitting one unit of pollution above the optimal level indicates instead that the cost of reducing pollution is too high compared to the benefit. The economic efficiency criterion does not involve consideration of distributional impacts (equity) or other ethical issues.
} 
value of pollution can be used to correct national accounts in a way that is consistent with national accounting practices. ${ }^{\text {ee }} 149$ In practice, there are often significant uncertainties in benefit and cost estimates of regulatory options, and factors other than economic efficiency (such as environmental justice) are considered in regulating emissions under federal environmental statutes.

Spatially differentiated cost estimates of air pollution:

The benefit per ton of reducing key air pollutants varies depending on the location of the emission reduction. For example, the marginal cost of emitting one unit of $\mathrm{SO}_{2}$ in a remote area may be lower than the marginal cost of the same unit of pollution emitted in a densely populated area, because emissions in populated areas generate greater health damages. However, the health impact attributable to a ton of $\mathrm{SO}_{2}$ will depend in part on the propensity of $\mathrm{SO}_{2}$ to form ammonium sulfate and contribute to the formation of $\mathrm{PM}_{2.5}$ and the baseline health status of the population living downwind, among other factors. Several studies have incorporated regional variations in the health impacts of $\mathrm{SO}_{2}$ and generated estimates of the marginal and average pollution costs that vary by location. 150151152153154

One study shows that metropolitan areas generate only $52 \%$ of emissions by weight, but they experience $73 \%$ of damages. ${ }^{155}$ Rural areas generate $48 \%$ of emissions by weight, but only experience $27 \%$ of damages.

The economic value of mortality risk reductions:

Increased morbidity and mortality are responsible for the largest share of external costs of power generation. ${ }^{156} 157158$ One of the main reasons behind the large difference in pollution cost estimates in the literature is that different studies use different assumptions to monetize the value of increased mortality risk due to pollution.

The literature typically uses the Value of a Statistical Life (VSL) to provide an economic estimate of the cost of one additional death caused by air pollution. The VSL is an estimate of the value that an individual places on a marginal change in the likelihood of death. ${ }^{159}$ The EPA recommends using a VSL equal to \$7.4 million (\$2006), ${ }^{160}$ equivalent to \$8.6 million in \$2015. Some studies also use the value of a statistical life-year (VSLY) to adjust for age and account for years of expected life lost. ${ }^{161}$

\footnotetext{
ee The assumption is that the marginal cost of pollution is the price of the environmental damage, and the price of any good is equal to the marginal cost of producing that good. By multiplying physical environmental damages by their prices it is possible to calculate the aggregate economic loss due to pollution. This estimate can be subtracted from national account statistics to generate estimates of gross economic activity.
} 


\subsubsection{The Economic Cost of Air Pollution from Power Generation}

In this section we present estimates of the cost of air pollution from power generation from the literature published after the year 2000. Early studies of the external cost of power generation in the United States include Mendelsohn, Ottinger et al., and Krupnick and Burtraw. ${ }^{162} 163164$ We focus on studies that separate power generation from other sources of air pollution damages. We first provide an aggregate estimate of pollution costs and then we separate by fuel and by pollutant.

Aggregate estimates:

Electricity generation activities account for the largest fraction of the external effects of the electricity system. ${ }^{165}$ Recent studies estimate that the cost of air pollution from power generation in the United States, during the years 2002-2011, ranges between \$71 and \$223 billion per year (Table 2.11). ${ }^{166} 167168169$ However, more recent studies suggest that air pollution costs may be higher than previously estimated (Table 2.11). For example, the AP2 model, ${ }^{170}$ an udpated version of the air pollution impact model APEEP, ${ }^{171}$ estimates costs three times larger than the older version of the model, in 2002 (Table 2.11).

There is evidence that pollution costs are declining over time (Table 2.11 and Table 2.12). Estimates based on historic pollution emissions reveal that total costs have declined by $25 \%$, from $\$ 223$ to $\$ 168$ billion per year. ${ }^{172}$ Air pollution costs per unit of electricity generated have declined by $30 \%$ from 2002 to 2011, from $\$ 87$ to $\$ 62$ per MWh. ${ }^{173}$ One study projects 2016 emissions and finds that costs may decline by as much as 35\% compared to 2005 (Table 2.11). ${ }^{174}$

Air pollution represents the largest external cost of power generation and $\mathrm{PM}_{2.5}$ health damages constitute the largest source of air pollution economic losses. ${ }^{175} 176177178$ For this reason some studies focus on $\mathrm{PM}_{2.5}$ health impacts alone. ${ }^{179} 180181182$ One study that focuses on health impacts alone finds air pollution costs equal to \$198-\$374 billion per year (Table 2.11), a cost estimate higher than what was found by studies that consider impacts in all sectors.

The differences across studies are in large part due to (1) the model used to estimate the spatial distribution and the chemical transformations of air emissions, (2) the VSL used in the study and (3) whether the VSL or VSLY is used. Tables 2.11 to 2.12 present detailed information on modelling choices to allow a more meaningful comparison of air pollution cost estimates.

The power sector contributes to societal well-being by providing a reliable source of power but it is also responsible for large external costs. One study finds that the gross external monetized damage (GED) caused by power generation is equal to $34 \%$ of its value added, without including the external cost of carbon dioxide emissions. ${ }^{183}$ 
Impacts by power plant type:

Monetized costs of air pollution from coal power generation range between $\$ 70$ and $\$ 183$ billion per year, using the standard \$8 million (2015 USD) estimate for the VSL (Table 2.11). About $95 \%$ of the physical and economic impact from air pollution are caused by coal power generation (Table 2.11) because it is the largest direct and indirect source of $\mathrm{PM}_{2.5}$, the most harmful pollutant for human health. The monetized cost per MWh of electricity from coal-fired power plants ranges between $\$ 36$ and $\$ 188,{ }^{\text {ff }}$ using standard assumptions on the VSL (Table 2.11). Differences in the sulfur content of coal, in emissions control technologies, in the age and in the geographic location all contribute to make pollution costs vary greatly across power plants. ${ }^{184}$ One study finds that the average cost across all coal power plants is equal to 50 $\$ / \mathrm{MWh}$, but the cleanest coal power plants have external costs less than $5.6 \$ / \mathrm{MWh}$ while the dirtiest coal power plants generate damages equal to $147 \$ / M W h .{ }^{g g} 185$ According to the same study, the $10 \%$ of coal-fired plants with the highest external costs account for about $40 \%$ of the total environmental damage from coal combustion. ${ }^{186}$ One study finds that coal power generation has a gross external damage ${ }^{\text {hh }}$ to value added (GED/VA) ratio equal to 2.2, meaning the pollution damages exceed the value added.i 187

Petroleum electricity generation causes monetized pollution costs that are about one third lower than coal electricity generation per $\mathrm{MWh}$, but overall generation is very small and thus petroleum power plants are responsible only for about $\$ 2$ to $\$ 6$ billion per year, using the standard \$8 million (2015 USD) estimate for the VSL. This corresponds to about 3\% of total power generation monetized costs of air pollution. One study finds that petroleum power generation has a GED/VA ratio equal to 5.1, an exceedingly high value that can be explained by the marginal role of petroleum generation.j 188

Air pollution from natural gas power generation causes monetized costs equal to about $\$ 1$ billion per year, corresponding to about $1 \%-2 \%$ of total monetized costs of air pollution, according to the studies cited in Table 2.12. Natural gas is widely used, but combustion of natural gas produces fewer harmful emissions compared to coal power generation. The

\footnotetext{
${ }^{\mathrm{ff}}$ Weighted average across all power plants, with weights equal to electricity generation. Table 2.9 also presents unweighted averages monetized costs per MWh produced by NRC (2010).

$\mathrm{gg}^{\text {th }}$ and $95^{\text {th }}$ percentile of the distribution, respectively. Unweighted average of costs per MWh across all power plants.

hh The gross external damage is defined by Muller, Mendelsohn and Nordhaus (2011, p. 1654) as marginal damages of emissions (the price) times the total quantity of emissions.

ii However, this does not necessarily mean that electricity generation from coal combustion would be uneconomical if external costs were fully incorporated. If the external cost were internalized in the cost of power generation, prices would change and the estimate of the value added of the power sector would probably increase.

${ }^{\mathrm{ij}}$ As for coal, a GDE/VA greater than unity does not necessarily mean that electricity generation from petroleum combustion would be uneconomical if external costs were fully incorporated.
} 
monetized cost per MWh of pollution from natural gas-fired power plants range between $\$ 1.8$ and $\$ 11.4$. As for coal power plants, the distribution of monetized costs of pollution from natural gas power plants is skewed. ${ }^{189}$ In 2005, the $50 \%$ of plants with lowest damages produced only $4 \%$ of aggregate damages. ${ }^{190}$ Conversely, the $10 \%$ most polluting natural gas power plants were responsible for $65 \%$ of air pollution from natural gas generation. ${ }^{191}$ The cleanest power plants generate monetized costs as low as $\$ 0.01 / \mathrm{MWh}$, while the most polluting plants cause costs equal to $\$ 6.2 / \mathrm{MWh}$. ${ }^{\mathrm{kk}} 192$ The GED/VA ratio for natural gas power generation is estimated to be equal to $0.34 .{ }^{193}$

kk The $5^{\text {th }}$ and the $95^{\text {th }}$ percentiles of the distribution, respectively. 
Table 2.11. Economic cost of air pollution from power generation, aggregate estimates. ${ }^{194}$

\begin{tabular}{|c|c|c|c|c|c|c|c|c|c|c|c|c|c|c|c|c|c|c|c|c|c|}
\hline \multirow[b]{2}{*}{ 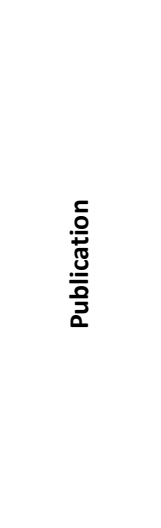 } & \multirow{2}{*}{ 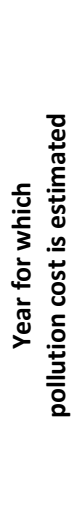 } & \multicolumn{2}{|c|}{$\begin{array}{l}\text { Cost of } \\
\text { pollution } \\
\text { from } \\
\text { power } \\
\text { generation }\end{array}$} & \multicolumn{2}{|c|}{$\begin{array}{c}\text { Cost } \\
\text { methodology }\end{array}$} & \multicolumn{5}{|c|}{ Sectors } & \multicolumn{6}{|c|}{$\begin{array}{l}\text { Air emissions } \\
\text { (primary) }\end{array}$} & \multicolumn{5}{|c|}{ Modeling options } \\
\hline & & 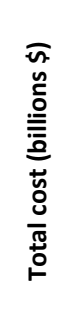 & 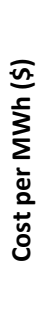 & 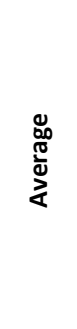 & 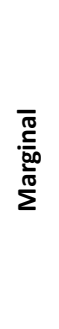 & $\begin{array}{l}\frac{5}{\frac{5}{\pi}} \\
\stackrel{\Xi}{I}\end{array}$ & $\frac{\vec{z}}{\frac{\vec{n}}{\frac{0}{n}}}$ & 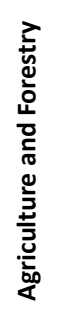 & 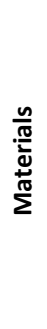 & 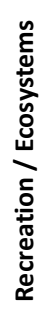 & ర $^{N}$ & $0^{x}$ & $\sum_{\substack{n \\
\stackrel{n}{N}}}^{\stackrel{n}{n}}$ & $\sum_{a}^{n}$ & $\frac{m}{2}$ & ๖ & 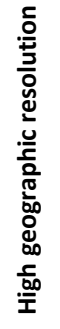 & 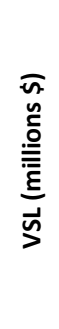 & $\begin{array}{l}\bar{d} \\
\text { ¿ } \\
\Sigma\end{array}$ & 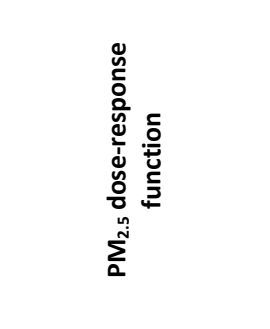 & 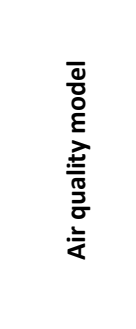 \\
\hline MMN (2011) & 2002 & 75 & & & $\checkmark$ & $\checkmark$ & $\checkmark$ & $\checkmark$ & $\checkmark$ & $\checkmark$ & $\checkmark$ & $\checkmark$ & $\checkmark$ & $\checkmark$ & $\checkmark$ & $\checkmark$ & $\checkmark$ & 8.0 & APEEP & Pope et al. (2002) 195 & RF - CMAQ \\
\hline JM (2016) & 2002 & 223 & 87 & & $\checkmark$ & $\checkmark$ & $\checkmark$ & $\checkmark$ & $\checkmark$ & $\checkmark$ & $\checkmark$ & $\checkmark$ & $\checkmark$ & $\checkmark$ & $\checkmark$ & $\checkmark$ & $\checkmark$ & 8.0 & $\mathrm{AP2}$ & Pope et al. (2002) & RF - CMAQ \\
\hline NRC (2010) & 2005 & 71 & 36 & & $\checkmark$ & $\checkmark$ & $\checkmark$ & $\checkmark$ & $\checkmark$ & $\checkmark$ & $\checkmark$ & $\checkmark$ & $\checkmark$ & $\checkmark$ & & & $\checkmark$ & 8.0 & APEEP & Pope et al. (2002) & $\mathrm{RF}-\mathrm{CMAQ}$ \\
\hline$J M(2016)$ & 2005 & 206 & 74 & & $\checkmark$ & $\checkmark$ & $\checkmark$ & $\checkmark$ & $\checkmark$ & $\checkmark$ & $\checkmark$ & $\checkmark$ & $\checkmark$ & $\checkmark$ & $\checkmark$ & $\checkmark$ & $\checkmark$ & 8.0 & AP2 & Pope et al. (2002) & RF - CMAQ \\
\hline FBF (2012) & 2005 & 374 & & $\checkmark$ & & $\checkmark$ & & & & & $\checkmark$ & $\checkmark$ & & $\checkmark$ & & & $\checkmark$ & 9.1 & & & RF-CAMx \\
\hline FBF (2012) & 2005 & 304 & & $\checkmark$ & & $\checkmark$ & & & & & $\checkmark$ & & & & & & $\checkmark$ & 9.1 & & (2009), ${ }^{196}$ & RF-CAMx \\
\hline FBF (2012) & 2005 & 15 & & $\checkmark$ & & $\checkmark$ & & & & & & $\checkmark$ & & & & & $\checkmark$ & 9.1 & & Laden et al. (2006) 197 & RF-CAMx \\
\hline FBF (2012) & 2005 & 55 & & $\checkmark$ & & $\checkmark$ & & & & & & & & $\checkmark$ & & & $\checkmark$ & 9.1 & & & RF-CAMx \\
\hline$J M(2016)$ & 2008 & 177 & 78 & & $\checkmark$ & $\checkmark$ & $\checkmark$ & $\checkmark$ & $\checkmark$ & $\checkmark$ & $\checkmark$ & $\checkmark$ & $\checkmark$ & $\checkmark$ & $\checkmark$ & $\checkmark$ & $\checkmark$ & 8.0 & AP2 & Pope et al. (2002) & RF - CMAQ \\
\hline FBF (2012) & 2016 & 198 & & $\checkmark$ & & $\checkmark$ & & & & & $\checkmark$ & $\checkmark$ & & $\checkmark$ & & & $\checkmark$ & 10 & & & RF-CAMx \\
\hline FBF (2012) & 2016 & 144 & & $\checkmark$ & & $\checkmark$ & & & & & $\checkmark$ & & & & & & $\checkmark$ & 10 & & Krewski et al (2009), & RF-CAMx \\
\hline FBF (2012) & 2016 & 10 & & $\checkmark$ & & $\checkmark$ & & & & & & $\checkmark$ & & & & & $\checkmark$ & 10 & & Laden et al. (2006) & RF-CAMx \\
\hline FBF (2012) & 2016 & 44 & & $\checkmark$ & & $\checkmark$ & & & & & & & & $\checkmark$ & & & $\checkmark$ & 10 & & & RF-CAMx \\
\hline$J M(2016)$ & 2011 & 168 & 62 & & $\checkmark$ & $\checkmark$ & $\checkmark$ & $\checkmark$ & $\checkmark$ & $\checkmark$ & $\checkmark$ & $\checkmark$ & $\checkmark$ & $\checkmark$ & $\checkmark$ & $\checkmark$ & $\checkmark$ & 8.0 & AP2 & Pope et al. (2002) & RF - CMAQ \\
\hline
\end{tabular}

Notes: All dollar figures have been converted to $2015 \$$ to allow for comparison across studies. MMN (2011): Muller, Mendelsohn, and Nordhaus (2011). ${ }^{198}$ JM (20016): Jaramillo and Muller (2016). ${ }^{199}$ NRC (2010): National Research Council (2010). 200 FBF (2012): Fann, Baker and Fulcher (2012). ${ }^{201}$ Cost estimates for FBF (2012) obtained by combining data in Fig 2 and Table 1. MMN (2011) and NRC (2010) use reduced functional forms calibrated to a baseline prediction of $\mathrm{PM}_{2.5}$ and $\mathrm{O}_{3}$ produced by CMAQ. 
Table 2.12 Economic cost of air pollution from power generation, by power plant type.

\begin{tabular}{|c|c|c|c|c|c|c|c|c|c|c|c|c|c|c|c|c|c|c|c|c|c|}
\hline \multirow[b]{2}{*}{ 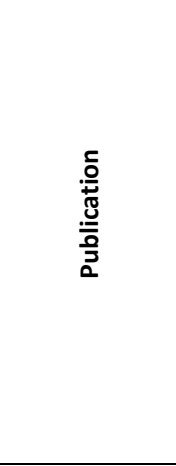 } & \multirow{2}{*}{ 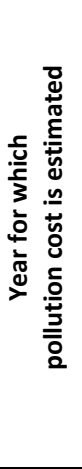 } & \multicolumn{2}{|c|}{$\begin{array}{l}\text { Cost of } \\
\text { pollution } \\
\text { from power } \\
\text { generation }\end{array}$} & \multicolumn{2}{|c|}{$\begin{array}{c}\text { Cost } \\
\text { methodology }\end{array}$} & \multicolumn{5}{|c|}{ Sectors } & \multicolumn{5}{|c|}{ Air emissions } & \multicolumn{6}{|c|}{ Modeling options } \\
\hline & & 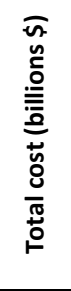 & 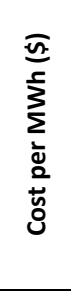 & 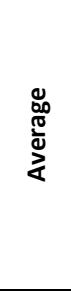 & $\begin{array}{l}\bar{\pi} \\
\stackrel{5}{\frac{5}{00}} \\
\sum \\
\sum\end{array}$ & 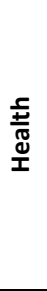 & $\begin{array}{l}\frac{3}{\bar{t}} \\
\frac{\overline{6}}{\bar{n}} \\
\frac{5}{5}\end{array}$ & 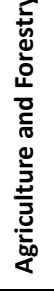 & 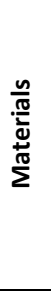 & 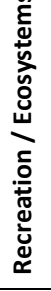 & ᄋ̃ & $0^{x}$ & 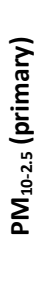 & 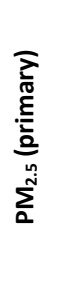 & $\frac{x}{2}$ & 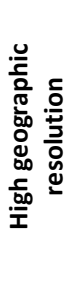 & 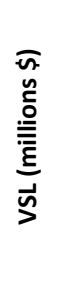 & जั & $\begin{array}{l}\bar{d} \\
\overline{0} \\
\Sigma\end{array}$ & 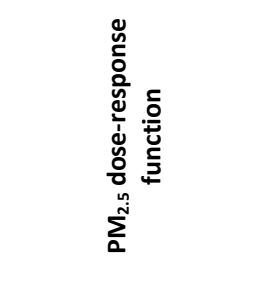 & 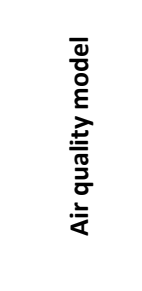 \\
\hline \multicolumn{22}{|l|}{ Coal } \\
\hline LBS (2009) & 1999 & & 188 & & $\checkmark$ & $\checkmark$ & & & & & $\checkmark$ & $\checkmark$ & & $\checkmark$ & & $\checkmark$ & 8.2 & & & Dockery et al. (1993) & \\
\hline MMN (2011) & 2002 & 72 & 37.5 & & $\checkmark$ & $\checkmark$ & $\checkmark$ & $\checkmark$ & $\checkmark$ & $\checkmark$ & $\checkmark$ & $\checkmark$ & $\checkmark$ & $\checkmark$ & $\checkmark \checkmark$ & $\checkmark$ & 8.0 & $\checkmark$ & APEEP & Pope et al. (2002) & RF - CMAQ \\
\hline $\operatorname{MMN}(2011)^{*}$ & 2002 & 157 & 82.4 & & $\checkmark$ & $\checkmark$ & $\checkmark$ & $\checkmark$ & $\checkmark$ & $\checkmark$ & $\checkmark$ & $\checkmark$ & $\checkmark$ & $\checkmark$ & $\checkmark \checkmark$ & $\checkmark$ & 8.0 & $\checkmark$ & APEEP & Laden et al. (2006) & RF - CMAQ \\
\hline MMN (2011) * & 2002 & 183 & 96.1 & & $\checkmark$ & $\checkmark$ & $\checkmark$ & $\checkmark$ & $\checkmark$ & $\checkmark$ & $\checkmark$ & $\checkmark$ & $\checkmark$ & $\checkmark$ & $\checkmark \checkmark$ & $\checkmark$ & 8.0 & & APEEP & Pope et al. (2002) & $\mathrm{RF}-\mathrm{CMAQ}$ \\
\hline $\operatorname{MMN}(2011)^{*}$ & 2002 & 25 & 13.3 & & $\checkmark$ & $\checkmark$ & $\checkmark$ & $\checkmark$ & $\checkmark$ & $\checkmark$ & $\checkmark$ & $\checkmark$ & $\checkmark$ & $\checkmark$ & $\checkmark \checkmark$ & $\checkmark$ & 2.7 & $\checkmark$ & APEEP & Pope et al. (2002) & RF - CMAQ \\
\hline MMN (2011) * & 2002 & 302 & 158 & & $\checkmark$ & $\checkmark$ & $\checkmark$ & $\checkmark$ & $\checkmark$ & $\checkmark$ & $\checkmark$ & $\checkmark$ & $\checkmark$ & $\checkmark$ & $\checkmark v$ & $\checkmark$ & 13 & $\checkmark$ & APEEP & Pope et al. (2002) & RF - CMAQ \\
\hline NRC (2010) & 2005 & 70 & 36.1 & & $\checkmark$ & $\checkmark$ & $\checkmark$ & $\checkmark$ & $\checkmark$ & $\checkmark$ & $\checkmark$ & $\checkmark$ & $\checkmark$ & $\checkmark$ & & $\checkmark$ & 8.0 & & APEEP & Pope et al. (2002) & RF - CMAQ \\
\hline NRC (2010) & 2005 & 26 & 13.5 & & $\checkmark$ & $\checkmark$ & $\checkmark$ & $\checkmark$ & $\checkmark$ & $\checkmark$ & $\checkmark$ & $\checkmark$ & $\checkmark$ & $\checkmark$ & & $\checkmark$ & 2.7 & & APEEP & Pope et al. (2002) & RF - CMAQ \\
\hline NRC (2010) & 2005 & 210 & 108 & & $\checkmark$ & $\checkmark$ & $\checkmark$ & $\checkmark$ & $\checkmark$ & $\checkmark$ & $\checkmark$ & $\checkmark$ & $\checkmark$ & $\checkmark$ & & $\checkmark$ & 8.0 & & APEEP & Dockery et al. (1993) & RF - CMAQ \\
\hline NRC (2010) ${ }^{* *}$ & 2005 & & 49.6 & & $\checkmark$ & $\checkmark$ & $\checkmark$ & $\checkmark$ & $\checkmark$ & $\checkmark$ & $\checkmark$ & $\checkmark$ & $\checkmark$ & $\checkmark$ & & $\checkmark$ & 8.0 & & APEEP & Pope et al. (2002) & RF - CMAQ \\
\hline NRC (2010) ** & 2005 & & 42.9 & & $\checkmark$ & $\checkmark$ & $\checkmark$ & $\checkmark$ & $\checkmark$ & $\checkmark$ & $\checkmark$ & & & & & $\checkmark$ & 8.0 & & APEEP & Pope et al. (2002) & RF - CMAQ \\
\hline NRC $(2010)^{* *}$ & 2005 & & 3.83 & & $\checkmark$ & $\checkmark$ & $\checkmark$ & $\checkmark$ & $\checkmark$ & $\checkmark$ & & $\checkmark$ & & & & $\checkmark$ & 8.0 & & APEEP & Pope et al. (2002) & RF - CMAQ \\
\hline NRC $(2010)^{* *}$ & 2005 & & 3.38 & & $\checkmark$ & $\checkmark$ & $\checkmark$ & $\checkmark$ & $\checkmark$ & $\checkmark$ & & & $\checkmark$ & & & $\checkmark$ & 8.0 & & APEEP & Pope et al. (2002) & RF - CMAQ \\
\hline $\operatorname{NRC}(2010){ }^{* *}$ & 2005 & & 0.19 & & $\checkmark$ & $\checkmark$ & $\checkmark$ & $\checkmark$ & $\checkmark$ & $\checkmark$ & & & & $\checkmark$ & & $\checkmark$ & 8.0 & & APEEP & Pope et al. (2002) & RF - CMAQ \\
\hline \multicolumn{22}{|l|}{ Natural Gas } \\
\hline MMN (2011) & 2002 & 1.2 & 11.4 & & $\checkmark$ & $\checkmark$ & $\checkmark$ & $\checkmark$ & $\checkmark$ & $\checkmark$ & $\checkmark$ & $\checkmark$ & $\checkmark$ & $\checkmark$ & $\checkmark v$ & $\checkmark$ & 8.0 & $\checkmark$ & APEEP & Pope et al. (2002) & RF - CMAQ \\
\hline NRC (2010) & 2005 & 0.8 & 1.80 & & $\checkmark$ & $\checkmark$ & $\checkmark$ & $\checkmark$ & $\checkmark$ & $\checkmark$ & $\checkmark$ & $\checkmark$ & $\checkmark$ & $\checkmark$ & & $\checkmark$ & 8.0 & & APEEP & Pope et al. (2002) & RF - CMAQ \\
\hline $\operatorname{NRC}(2010)^{*}$ & 2005 & & 4.85 & & $\checkmark$ & $\checkmark$ & $\checkmark$ & $\checkmark$ & $\checkmark$ & $\checkmark$ & $\checkmark$ & $\checkmark$ & $\checkmark$ & $\checkmark$ & & $\checkmark$ & 8.0 & & APEEP & Pope et al. (2002) & RF - CMAQ \\
\hline $\operatorname{NRC}(2010)^{*}$ & 2005 & & 0.20 & & $\checkmark$ & $\checkmark$ & $\checkmark$ & $\checkmark$ & $\checkmark$ & $\checkmark$ & $\checkmark$ & & & & & $\checkmark$ & 8.0 & & APEEP & Pope et al. (2002) & RF - CMAQ \\
\hline $\operatorname{NRC}(2010)^{*}$ & 2005 & & 2.59 & & $\checkmark$ & $\checkmark$ & $\checkmark$ & $\checkmark$ & $\checkmark$ & $\checkmark$ & & $\checkmark$ & & & & $\checkmark$ & 8.0 & & APEEP & Pope et al. (2002) & RF - CMAQ \\
\hline $\operatorname{NRC}(2010)^{*}$ & 2005 & & 0.10 & & $\checkmark$ & $\checkmark$ & $\checkmark$ & $\checkmark$ & $\checkmark$ & $\checkmark$ & & & $\checkmark$ & & & $\checkmark$ & 8.0 & & APEEP & Pope et al. (2002) & RF - CMAQ \\
\hline $\operatorname{NRC}(2010)^{*}$ & 2005 & & 1.92 & & $\checkmark$ & $\checkmark$ & $\checkmark$ & $\checkmark$ & $\checkmark$ & $\checkmark$ & & & & $\checkmark$ & & $\checkmark$ & 8.0 & & APEEP & Pope et al. (2002) & RF - CMAQ \\
\hline
\end{tabular}




\begin{tabular}{|c|c|c|c|c|c|c|c|c|c|c|c|c|c|c|c|c|c|c|c|c|c|}
\hline \multirow[b]{2}{*}{ 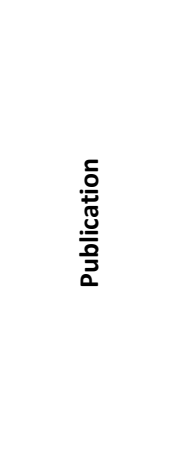 } & \multirow{2}{*}{ 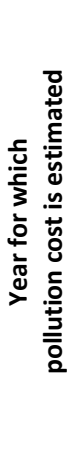 } & \multicolumn{2}{|c|}{$\begin{array}{l}\text { Cost of } \\
\text { pollution } \\
\text { from power } \\
\text { generation }\end{array}$} & \multicolumn{2}{|c|}{$\begin{array}{c}\text { Cost } \\
\text { methodology }\end{array}$} & \multicolumn{5}{|c|}{ Sectors } & \multicolumn{5}{|c|}{ Air emissions } & \multicolumn{6}{|c|}{ Modeling options } \\
\hline & & 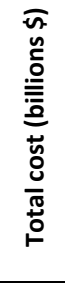 & 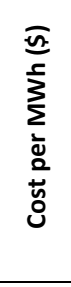 & 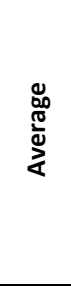 & 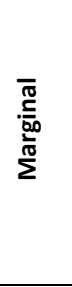 & $\begin{array}{l}\frac{5}{\frac{5}{\pi}} \\
\frac{\dddot{x}}{x}\end{array}$ & 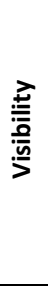 & 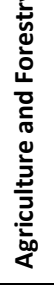 & 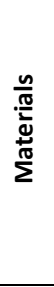 & 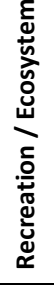 & Оั & $0^{x}$ & 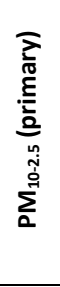 & 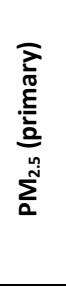 & I & 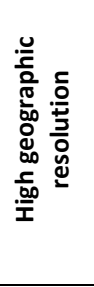 & 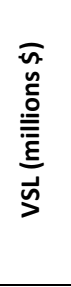 & जั & 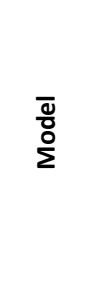 & 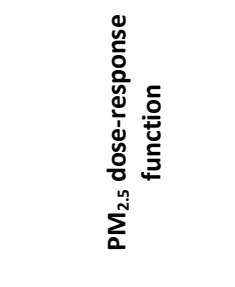 & 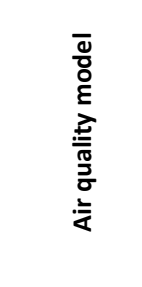 \\
\hline Petroleum & & & & & & & & & & & & & & & & & & & & & \\
\hline MMN (2011) & 2002 & 2 & 27.2 & & $\checkmark$ & $\checkmark$ & $\checkmark$ & $\checkmark$ & $\checkmark$ & $\checkmark$ & $\checkmark$ & $\checkmark$ & $\checkmark$ & $\checkmark$ & $\checkmark v$ & $\checkmark$ & 8.0 & $\checkmark$ & APEEP & Pope et al. (2002) & $\mathrm{RF}-\mathrm{CMAQ}$ \\
\hline $\operatorname{MMN}(2011)^{*}$ & 2002 & 5 & 58.2 & & $\checkmark$ & $\checkmark$ & $\checkmark$ & $\checkmark$ & $\checkmark$ & $\checkmark$ & $\checkmark$ & $\checkmark$ & $\checkmark$ & $\checkmark$ & $\checkmark \checkmark$ & $\checkmark$ & 8.0 & $\checkmark$ & APEEP & Laden et al. (2006) & RF - CMAQ \\
\hline $\operatorname{MMN}(2011)^{*}$ & 2002 & 6 & 69.3 & & $\checkmark$ & $\checkmark$ & $\checkmark$ & $\checkmark$ & $\checkmark$ & $\checkmark$ & $\checkmark$ & $\checkmark$ & $\checkmark$ & $\checkmark$ & $\checkmark v$ & $\checkmark$ & 8.0 & & APEEP & Pope et al. (2002) & RF - CMAQ \\
\hline $\operatorname{MMN}(2011)^{*}$ & 2002 & 1 & 9.4 & & $\checkmark$ & $\checkmark$ & $\checkmark$ & $\checkmark$ & $\checkmark$ & $\checkmark$ & $\checkmark$ & $\checkmark$ & $\checkmark$ & $\checkmark$ & $\checkmark v$ & $\checkmark$ & 2.7 & $\checkmark$ & APEEP & Pope et al. (2002) & RF - CMAQ \\
\hline MMN (2011) * & 2002 & 4 & 43.8 & & $\checkmark$ & $\checkmark$ & $\checkmark$ & $\checkmark$ & $\checkmark$ & $\checkmark$ & $\checkmark$ & $\checkmark$ & $\checkmark$ & $\checkmark$ & $\checkmark v$ & $\checkmark$ & 13 & $\checkmark$ & APEEP & Pope et al. (2002) & RF - CMAQ \\
\hline
\end{tabular}

Notes: All dollar figures have been converted to $2015 \$$ to allow for comparison across studies. LBS (2009): Levy, Baxter and Schwartz (2009), 202 p. 1008, median across plants; note that LBS (2009) estimates are for the Mid-Atlantic Great Lakes regions only. MMN (2011): Muller, Mendelsohn, and Nordhaus (2011). ${ }^{203}$ MMN (2011)*: extrapolated from MMN (2011) Table 3, p. 1668 assuming constant VA and constant electricity generation. NRC (2010): National Research Council (2010), weighted average with weights equal to electricity generation; Coal: 406 plants, corresponding to $94.6 \%$ electricity generation; ${ }^{204}$ natural gas: 498 gas power plants $71 \%$ natural gas electricity generation. ${ }^{205} \mathrm{NRC}(2010)^{* *}$ : National Research Council (2010), unweighted average across all power plants. 206207 
Impacts by pollutant:

$\mathrm{PM}_{2.5}$ causes the largest fraction of monetized costs of air pollution per ton of emissions (Table 2.13). The estimated cost per ton of $P M_{2.5}$ vary greatly in the literature, from $\$ 32$ to $\$ 141$, as shown in Table 2.13. The average of the estimates in the reviewed studies is equal to $\$ 70 /$ ton and the median is equal to $\$ 60 /$ ton. One study finds that only $6 \%$ of total pollution by weight is $\mathrm{PM}_{2.5}$, but it accounts for $23 \%$ of damages. ${ }^{208} \mathrm{NO}$ x emissions account for $27 \%$ of emissions by weight but they generate only $8 \%$ of total damages. ${ }^{209}$ The cost per ton of $\mathrm{NO}_{\mathrm{x}}$ emission varies between $\$ 3$ and $\$ 17$ in the reviewed studies (Table 2.13). The median of the distribution is equal to $\$ 5 /$ ton and the mean is equal to $\$ 9 /$ ton (Table 2.13). $\mathrm{SO}_{2}$ is the largest source of economic damages from power plants because it is emitted in large quantities by coal power plants and is a precursor of $\mathrm{PM}_{2.5}$ the air pollutant with the largest negative health effects (Table 2.13 and Table 2.14). The reviewed studies indicate that the cost of $\mathrm{SO}_{2}$ emissions ranges between $\$ 17$ and $\$ 95$, with a mean across studies equal to $\$ 35 /$ ton and a median cost equal to $\$ 30 /$ ton (Table 2.13).

Table 2.11 and Table 2.12 provide estimates of the cost per MWh of electricity attributable to each pollutant, also by fuel type. $\mathrm{SO}_{2}$ emissions are the main source of air pollution monetized damages per unit of electricity generated using coal (Table 2.14). NOx emissions are instead the main source of monetized damages for electricity generated using natural gas.

Table 2.13. Economic cost of $\mathrm{PM}_{2.5}$ direct emissions and $\mathrm{PM}_{2.5}$ precursors (thousands of $\$$ per ton)

\begin{tabular}{|c|c|c|c|c|c|c|c|}
\hline & & & & $\mathrm{PM}_{2.5}$ & & & \\
\hline & 1999 & 2002 & 2005 & 2008 & 2011 & 2015 & 2016 \\
\hline \multicolumn{8}{|l|}{ FFH (2009) } \\
\hline LBS (2009) & 83.4 & & & & & & \\
\hline FBF (2012) & & & 108 & & & & 141 \\
\hline Buonocore et al. (2014) & & & 141 & & & & \\
\hline \multirow[t]{3}{*}{ JM (2016) } & & 35.1 & 32.3 & 36.4 & 37.6 & & \\
\hline & & & & $\mathrm{NO}_{\mathrm{x}}$ & & & \\
\hline & 1999 & 2002 & 2005 & 2008 & 2011 & 2015 & 2016 \\
\hline FFH (2009) & & & & & & 17.4 & \\
\hline LBS (2009) & 5.56 & & & & & & \\
\hline FBF (2012) & & & 4.01 & & & & 5.64 \\
\hline Buonocore et al. (2014) & & & 17.4 & & & & \\
\hline \multirow[t]{3}{*}{ JM (2016) } & & 3.26 & 3.10 & 4.67 & 4.34 & & \\
\hline & & & & $\mathrm{SO}_{2}$ & & & \\
\hline & 1999 & 2002 & 2005 & 2008 & 2011 & 2015 & 2016 \\
\hline FFH (2009) & & & & & & 94.9 & \\
\hline LBS (2009) & 22.0 & & & & & & \\
\hline FBF (2012) & & & 29.3 & & & & 38.0 \\
\hline Buonocore et al. (2014) & & & 30.4 & & & & \\
\hline JM (2016) & & 18.3 & 17.1 & 19.2 & 32.8 & & \\
\hline
\end{tabular}

Notes: All dollar figures have been converted to $2015 \$$ to allow for comparison across studies. $\mathrm{FFH}$ (2009): Fann, Fulcher and Hubbell. ${ }^{210}$ FBF (2012): Fann, Baker and Fulcher (2009). ${ }^{211}$ LBS (2009): Levy, Baxter and Schwartz (2009), ${ }^{212}$ median across plants; note that LBS (2009) estimates are for the MidAtlantic Great Lakes regions only. Buonocore et al. (2014): median estimate. ${ }^{213}$ JM (20016): Jaramillo and Muller (2016), ${ }^{214}$ Table S-4 of Supporting Information document. 
Impacts by region:

The recent literature uses models with high geographic resolution to study air pollution impacts and costs. However, regional estimates are usually not available. Two studies provide a summary of regional pollution monetized costs from power generation. The first study uses projected air emissions and other baseline developments for the year 2015 and estimates monetized costs of air pollution in nine U.S. metropolitan areas and at a national level. ${ }^{215}$ Table 2.11 displays estimated monetized costs per ton of selected $\mathrm{PM}_{2.5}$ precursor emissions from power generating units. The national cost is equal to $\$ 17 /$ ton for $N O_{x}$ emissions and equal to $\$ 95 /$ ton for $\mathrm{SO}_{2}$ (Table 2.12). The national averages hide substantial geographic heterogeneity. For example, one ton of $\mathrm{NO}$ x emissions causes monetized costs equal to $\$ 139,000 /$ ton in Seattle while the cost per ton in Chicago is equal to only $\$ 1,300$ (Table 2.14). One ton of $\mathrm{SO}_{2}$ emissions causes monetized costs equal to $\$ 405,000 /$ ton in the San Joaquin metropolitan area while the cost per ton in Seattle is equal to only $\$ 7300$ (Table 2.14). These large geographic differences are due to local geographic characteristics, the prevalent type of fuel type used in nearby power plants, the characteristics of the emitting source (e.g. height of the smokestacks), population density and other population characteristics. ${ }^{216}$

Table 2.14. The monetized cost of selected $\mathrm{PM}_{2.5}$ precursor emissions ${ }^{217}$

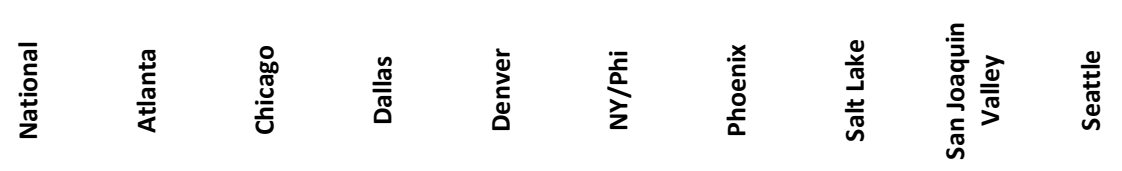

\begin{tabular}{lccccccccc}
\hline $\mathrm{SO}_{2}$ & 95 & 17 & 21 & & 7.4 & 86 & & 405 & 7.3 \\
$\mathrm{NO}$ & 17 & 9.1 & 1.3 & 39.4 & 3.7 & 1.7 & 13 & 32 & 139
\end{tabular}

Note: Thousands of dollars per ton of emission; dollar figures have been converted to $2015 \$$ using a GDP deflator.

In the Regulatory Impact Analysis for the Clean Power Plan Final Rule, the EPA assessed the health benefits of air pollution mitigation in $2020 .^{218}$ The analysis considers health impacts from $\mathrm{SO}_{2}, \mathrm{NO}_{x}$, directly emitted $\mathrm{PM}_{2.5}, \mathrm{NO}_{\mathrm{x}}$ as a precursor of $\mathrm{PM}_{2.5}$, and $\mathrm{NO}_{\mathrm{x}}$ as a precursor of groundlevel ozone for the year 2020. The estimates are reported in Table 2.15, at national level and for three large representative regions. Table 2.15 reveals that the health impacts vary substantially across different pollutants. $\mathrm{PM}_{2.5}$ generates the largest negative effects (Table 2.15). The health impacts also vary greatly geographically, due to many differences in local population, geography, power generation mix. 
Table 2.15. The health impacts of pollution from electricity generation utilities in three regions of the United States in the year $2020(2015 \$)^{219}$

\begin{tabular}{|c|c|c|c|c|c|}
\hline \multirow{2}{*}{ Pollutant } & \multirow{2}{*}{$\begin{array}{l}\text { Discount } \\
\text { Rate }\end{array}$} & \multirow{2}{*}{ National } & \multicolumn{3}{|c|}{ Regional } \\
\hline & & & East & West & California \\
\hline \multirow[t]{2}{*}{$\mathrm{SO}_{2}$} & $3 \%$ & 35.1 to 79.7 & 6.6 to 14.9 & 100.9 to 223.1 & 170 to 340 \\
\hline & $7 \%$ & 31.9 to 72.3 & 6 to 13.8 & 90.3 to 201.9 & 148.8 to 340 \\
\hline \multicolumn{6}{|l|}{ Directly emitted $\mathrm{PM}_{2.5}$} \\
\hline \multirow[t]{2}{*}{$(\mathrm{EC}+\mathrm{OC})$} & $3 \%$ & 148.8 to 340 & 28.7 to 63.8 & 393.2 to 881.9 & 297.5 to 605.7 \\
\hline & $7 \%$ & 138.1 to 308.1 & 25.5 to 57.4 & 350.6 to 786.3 & 265.6 to 605.7 \\
\hline \multirow[t]{2}{*}{$\begin{array}{l}\text { Directly emitted } \mathrm{PM}_{2.5} \\
\text { (crustal) }\end{array}$} & $3 \%$ & 24.4 to 55.3 & 11.7 to 26.6 & 77.6 to 170 & 116.9 to 233.8 \\
\hline & $7 \%$ & 22.3 to 49.9 & 10.5 to 23.4 & 70.1 to 159.4 & 100.9 to 233.8 \\
\hline \multirow[t]{2}{*}{$\mathrm{NO} \times$ (as $\mathrm{PM}_{2.5}$ ) } & $3 \%$ & 3.3 to 7.4 & 0.7 to 1.6 & 23.4 to 52.1 & 18.1 to 36.1 \\
\hline & $7 \%$ & 3 to 6.7 & 0.6 to 1.5 & 20.2 to 46.8 & 15.9 to 36.1 \\
\hline $\mathrm{NO}_{\mathrm{x}}$ (as Ozone) & $\mathrm{N} / \mathrm{A}$ & 6.9 to 29.8 & 2.1 to 9.5 & 14.9 to 62.7 & 7.9 to 32.9 \\
\hline
\end{tabular}

Notes: Thousands of dollars per ton of emission. All dollar figures have been converted to $2015 \$$ using a GDP deflator. This assessment includes separate benefits analysis for two categories of directly emitted particles: elemental carbon plus organic carbon $(E C+O C)$ and crustal. Crustal emissions are composed of compounds associated with minerals and metals from the earth's surface, including carbonates, silicates, iron, phosphates, copper, and zinc. The range of estimates reflects the range of epidemiology studies for avoided premature mortality for $\mathrm{PM}_{2.5}$ and ozone. The monetized co-benefits do not include reduced health effects from direct exposure to $\mathrm{NO}_{2}, \mathrm{SO}_{2}$, ecosystem effects, or visibility impairment. All fine particles are assumed to have equivalent health effects, but the benefit per ton estimates vary depending on the location and magnitude of their impact on $\mathrm{PM}_{2.5}$ concentrations, which drive population exposure. The monetized co-benefits incorporate the conversion from precursor emissions to ambient fine particles and ozone. Benefit-per-ton estimates for ozone are based on ozone season NOx emissions. Ozone cobenefits occur in analysis year, so they are the same for all discount rates.

The average cost of pollution from coal-fired power plants is equal to $4 \mathrm{c} / \mathrm{kWh} .{ }^{220}$ But differences in the sulfur content of coal, the presence of control technologies, and the vintage of the plant make the external cost per kWh vary greatly across power plants. The cleanest power plants have external costs less than $1 \&$ per $\mathrm{kWh}$. The dirtiest power plants lead to external costs greater than $12 \mathrm{c} / \mathrm{kWh}$. The $10 \%$ of coal-fired plants with the highest external costs account for about $40 \%$ of the total environmental damage from coal combustion.

The average cost of pollution from natural gas-fired power plants is equal to $0.2 \mathrm{c} / \mathrm{kWh} .{ }^{221} \mathrm{The}$ distribution of pollution from different sources is also uneven for natural gas power plants. Fifty percent of plants with the lowest damages produced only $4 \%$ of aggregate damages. Conversely, the $10 \%$ most polluting natural gas power plants were responsible for $65 \%$ of air pollution from natural gas generation. This translates into external costs per kWh of $0.05 \mathrm{c}$ in the cleanest power plants and $1 \mathrm{c}$ in the most polluting plants. 


\subsubsection{Air Quality Co-Benefits of $\mathrm{CO}_{2}$ Emissions Regulation}

Regulation of carbon dioxide emissions from power plants is expected to reduce emissions of other air pollutants, creating additional health and environmental benefits in addition to the avoided climate change impacts. These additional benefits of carbon emissions regulation are called ancillary benefits. ${ }^{222}$

Emissions of air pollutants are expected to decline because policies to reduce carbon dioxide emissions create incentives to increase efficiency of power generation and energy end-use, and to switch from coal to natural gas, nuclear and renewable power, which have lower carbon dioxide emissions and lower emissions of local air pollutants.

For example, the EPA Regulatory Impact Analysis for the Clean Power Plan Final Rule finds large economic co-benefits of carbon emission reductions. ${ }^{223}$ With a $3 \%$ discount rate, monetized climate and health co-benefits in 2030 range between $\$ 18$ and $\$ 40$ billion per year.

Table 2.16. Combined Estimates of Climate Benefits and Health Co-benefits from Clean Power Plan $^{224}$

\begin{tabular}{|c|c|c|c|c|c|}
\hline \multirow[b]{3}{*}{2020} & \multicolumn{5}{|c|}{ Benefits per abated ton of $\mathrm{CO}_{2}(\$ /$ short ton $2015 \$)$} \\
\hline & \multirow{2}{*}{$\begin{array}{l}\text { Climate benefits } \\
\$ 43.00\end{array}$} & \multicolumn{2}{|c|}{ Health benefits (range) } & \multicolumn{2}{|c|}{ Total (range) } \\
\hline & & $\$ 26.00$ & $\$ 62.00$ & $\$ 69.00$ & $\$ 105.00$ \\
\hline 2025 & $\$ 48.00$ & $\$ 28.00$ & $\$ 68.00$ & $\$ 76.00$ & $\$ 117.00$ \\
\hline \multirow[t]{3}{*}{2030} & $\$ 51.00$ & $\$ 31.00$ & $\$ 72.00$ & $\$ 82.00$ & $\$ 123.00$ \\
\hline & \multicolumn{5}{|c|}{ Total benefits (billions of $2015 \$$ ) } \\
\hline & Climate benefits & Health & ange) & Total (ra & \\
\hline 2020 & $\$ 3.50$ & $\$ 2.10$ & $\$ 5.10$ & $\$ 5.60$ & $\$ 8.60$ \\
\hline 2025 & $\$ 13.00$ & $\$ 7.40$ & $\$ 18.00$ & $\$ 20.00$ & $\$ 31.00$ \\
\hline 2030 & $\$ 21.00$ & $\$ 13.00$ & $\$ 30.00$ & $\$ 34.00$ & $\$ 51.00$ \\
\hline
\end{tabular}

Notes: All dollar figures have been converted to $2015 \$$ using a GDP deflator. Benefit estimates for massbased approach. Discount rate used for both climate benefits and co-benefits: $3 \%$. Climate benefits are based on reductions in $\mathrm{CO}_{2}$ emissions. Co-benefits are based on regional benefit-per-ton estimates. Ozone co-benefits occur in analysis year, so they are the same for all discount rates. The health cobenefits reflect the sum of the $\mathrm{PM}_{2.5}$ and ozone co-benefits and reflect the range based on adult mortality functions in the literature. The monetized health co-benefits do not include reduced health effects from reductions in directly emitted $\mathrm{PM}_{2.5}$, direct exposure to $\mathrm{NOx}, \mathrm{SO}_{2}$, and HAP; ecosystem effects; or visibility impairment. See Chapter 4 of EPA (2015) for more information. ${ }^{225}$ 
Most studies are in line with the EPA results (Table 2.17)." Ancillary benefits range between \$3.5 and \$131 per ton of $\mathrm{CO}_{2}$-eq abated (Table 2.17), including all emissions sources. The literature generally finds that most of the benefits come from reduced emissions of $\mathrm{PM}_{2.5}$ from coal power plants, usually not less than $\$ 30$ per ton of $\mathrm{CO}_{2}$-eq abatement. ${ }^{226} 227228$ The ancillary benefits per kWh of reduction in coal power emissions are comparable to estimates of the social cost of carbon (SCC $)^{\mathrm{mm}}$ by the Interagency Working Group. ${ }^{\text {nn } 229}$ Natural gas power generation results in much less air polluting emissions than coal, and the local ancillary benefits of reducing natural gas generation are smaller than some published estimates of carbon damages. ${ }^{230}$ The co-benefits tend to increase less than costs when mitigation becomes more stringent. 231232

Table 2.17. Estimates of Health Co-Benefits of Mitigation Policies ${ }^{233}$

\begin{tabular}{|c|c|c|c|c|c|}
\hline & \multirow{2}{*}{ Country } & \multirow{2}{*}{$\begin{array}{c}\text { Carbon Tax } \\
\left(\$ / \mathrm{tCO}_{2}\right)\end{array}$} & \multicolumn{3}{|c|}{ Co-benefits $\left(\$ / \mathrm{tCO}_{2}\right)$} \\
\hline & & & Min & Median & Max \\
\hline \multirow[t]{2}{*}{ Burtraw et al. (2003) ${ }^{234}$} & US & 35 & 17 & & 20 \\
\hline & US & 106 & 17 & & \\
\hline Nemet (2010) 235 & $\begin{array}{l}\text { Developed } \\
\text { countries }\end{array}$ & & 2 & 34 & 142 \\
\hline \multirow[t]{2}{*}{ Groosman et al. (2011) 236} & US & 11 & 4 & & 11 \\
\hline & US & 65 & 22 & & 87 \\
\hline \multirow[t]{4}{*}{ West et al. $(2013)^{237}$} & Global & & 60 & & 453 \\
\hline & Europe/US & & 36 & & 716 \\
\hline & China & & 84 & & 1,002 \\
\hline & India & & 24 & & 477 \\
\hline
\end{tabular}

Notes: All dollar figures have been converted to $2015 \$$ to allow for comparison across studies.

\footnotetext{
"One recent exception is the West et al. (2013) study, which finds much larger co-benefits than previous studies. This is in part explained by the use of a new relationship for chronic mortality. West et al. (2013) also find that the monetized mortality co-benefits exceed the median carbon price in virtually all world regions in their medium stringency mitigation scenario. In East Asia the co-benefits are 10 to 70 times the median carbon price. These estimates are outliers from previous studies.

$\mathrm{mm}$ The social cost of carbon is an estimate of the economic damages associated with a small increase in carbon dioxide $\left(\mathrm{CO}_{2}\right)$ emissions, conventionally one metric ton, in a given year. See https://www3.epa.gov/climatechange/EPAactivities/economics/scc.html for more information.

$\mathrm{nn}$ The ancillary benefits per kWh would typically be higher than carbon benefits per kWh based on the average SCC at a $5 \%$ discount rate. The other three SCC estimates (avg at 3\% and $2.5 \%, 95^{\text {th }}$ percentile at $3 \%$ ) yield higher estimates of carbon benefits.
} 


\section{IMPACTS TO LAND AND ECOLOGY}

The land use footprint of electricity infrastructures and associated operations has a range of direct impacts to ecosystems and society, more broadly. The magnitude of these impacts depends on how the infrastructure affects endangered species, involves sensitive ecological areas, impacts cultural or historic resources, gives rise to visual or aesthetic concerns, or opens new areas to development. ${ }^{238}$ Chapters $7^{239}$ and $9^{240}$ of the first installment of the Quadrennial Energy Review (QER) - which focused on energy transmission, storage and distributiondiscussed many of these issues and some highlights from those chapters are summarized in this section. Since the scope of this report also includes electricity generation, additional impacts and issues are discussed, including the land use footprints of electric generation facilities.

An important finding from the first installment of the QER is that while expanding transmission and distribution infrastructure can pose environmental challenges, building new infrastructure can also help to enable significant net environmental benefits. For this reason, agencies across the federal government are engaged in several initiatives to modernize the Federal Government's role with respect to electric transmission permitting and project review. ${ }^{241}$

This section begins by discussing considerations that are common to the land use and ecological impacts of electricity infrastructure. This includes descriptions of the land use requirements and ecological impacts of different types of power plants and transmission and distribution infrastructure. The chapter ends with a discussion of issues related to the siting and permitting of electricity infrastructure and a brief summary of ongoing efforts to modernize policies and approaches to planning and permitting new infrastructure.

It is worth acknowledging upfront that the level of detail is not always even across generation technology types. In many cases, more detailed discussions reflect the greater availability of current data and information rather than the relative degree of environmental impact. It is also worth noting that while this Environment baseline volume generally does not consider life cycle environmental impacts, some exceptions are made in this section, because published data and information on the land use footprints of electricity infrastructure are sometimes only available on a full life cycle basis. 


\subsection{LAND-USE AND ECOLOGICAL IMPACTS OF POWER GENERATION}

\subsubsection{Land Use Requirements of Power Generation}

Land use requirements for power generation reflect significant differences between the various types of infrastructure and operational requirements. The footprints of the power plants themselves are relatively small for fossil and nuclear power and relatively larger for renewables. However, from a life cycle standpoint, with the exception of bioenergy, land use requirements for renewable energy are dominated by the operational footprint of the generation facilities, while upstream impacts are relatively minor. For non-renewable power generation options, the opposite is generally true, when the upstream impacts of fuel resource extraction is accounted for.

Table 3.1 describes the land-use requirements of renewable energy technologies, based on NREL's 2012 Renewable Electricity Futures Study. It should be noted that, although the land use of wind energy is often expressed as the total area of a wind power plant, only a small fraction (typically less than 5\%) of that area is physically disturbed by turbine foundations, access roads, or other infrastructure while the remaining area can often be available for other productive uses, such as farming or ranching. For CSP and PV, the land-use footprint of the power plant can be much higher, but the land-use intensity of these facilities is also much greater, with a larger fraction of the total area occupied by plant infrastructure. ${ }^{\circ 0}$ The report suggests that more consistent methodologies are necessary to evaluate the relative impact of energy generation land use in order to provide a more direct comparison among these technologies.

oo "Land use factor" measures the generation capacity per land area of a typical power plant facility (e.g., $\mathrm{MW} / \mathrm{km}^{2}$ ). "Land use intensity" measures the portion of the land area within a power plant facility that is disturbed or directly occupied by the plant's infrastructure and related operations. 
Table 3.1. Land-Use Requirements of Renewable Technologies 242

\begin{tabular}{|c|c|c|}
\hline Renewable Technology & Land Use Footprint & Notes \\
\hline Biopower & $25,800 \mathrm{GJ} / \mathrm{km}^{2} / \mathrm{yr}$ & $\begin{array}{l}\text { Land-use factor uses the midrange estimate for } \\
\text { switchgrass. Other waste and residue feedstocks } \\
\text { are assumed to have no incremental land use } \\
\text { demands. }\end{array}$ \\
\hline Hydropower & $1,000 \mathrm{MW} / \mathrm{km}^{2}$ & $\begin{array}{l}\text { Assumed only run-of-river facilities, with land use } \\
\text { based only on facility civil works with no flooded } \\
\text { area. }\end{array}$ \\
\hline Wind (land-based) & $5 \mathrm{MW} / \mathrm{km}^{2}$ & $\begin{array}{l}\text { Most of the land occupied by land-based wind } \\
\text { power plants can be used for other purposes; } \\
\text { actual physical disruption for all related } \\
\text { infrastructure for land-based projects is } \\
\text { approximately } 5 \% \text { of total. }\end{array}$ \\
\hline Utility Scale PV & $50 \mathrm{MW} / \mathrm{km}^{2}$ & Direct land use of modules and inverters \\
\hline Distributed Rooftop PV & 0 & $\begin{array}{l}\text { Systems installed on rooftops do not compete } \\
\text { with other land uses and no incremental land use } \\
\text { is assumed here. }\end{array}$ \\
\hline CSP & $31 \mathrm{MW} / \mathrm{km}^{2}$ & $\begin{array}{l}\text { Overall land occupied by CSP solar collection } \\
\text { fields (excluding turbine, storage, and other site } \\
\text { works beyond mirrors). }\end{array}$ \\
\hline Geothermal & $500 \mathrm{MW} / \mathrm{km}^{2}$ & Direct land use of plant, wells and pipelines. \\
\hline
\end{tabular}

Land-use requirements vary across renewable technologies, with the largest land-use per capacity coming from wind and utility-scale solar PV and CSP.

There is very limited published literature comparing land use impacts across all electricity generation technologies. ${ }^{243}$ One exception is a 2009 peer-reviewed study, by Fthenakis and Kim, which normalized land requirements during the life cycles of conventional- and renewableenergy technology options. ${ }^{244}$ Their analysis found that the PV cycle requires the least amount of land among renewable-energy options, while the biomass cycle requires the largest. The study also concluded that the land requirement of ground-mounted PV systems in areas with high quality solar resources is no greater than that of coal-based fuel cycles, which require reclaiming mine lands and securing additional lands for waste disposal.

Details of land use for various generation technologies are discussed below. For all technology types, the siting of power plants involves transforming the existing landscape, removing soil and ground vegetation and the potential for erosion and sedimentation loading to waterways during construction. The Electricity Generation baseline volume ${ }^{245}$ includes addition discussion of upstream impacts, such as mining, and the Solid Waste baseline volume ${ }^{246}$ covers some of the downstream impacts associated with the management and disposal of solid waste from coal and nuclear facilities. 
Coal:

The coal fuel cycle affects the land use directly and indirectly during mining, beneficiation, electricity generation, and waste storage. For coal plant operations, land is required for the powerhouse, railroad switchyard and/ or dock for barge access, smoke stacks, precipitators, pedestrian and vehicle traffic, coal storage, and cooling towers. Space is also needed for coal ash storage, typically in retention ponds. The land use footprint of a 1,000 MW plant in the United States requires between 330 and 1000 acres, or 6-18 m²/GWh of transformed land based on a capacity factor of $0.85 .^{247}$

To maintain reliable fuel supplies, coal plants require access to railroad tracks and/or barge traffic on waterways. Also, coal generators drive steam turbines and require access to a reliable water source for cooling. Upstream, coal mining is conducted both on the surface and underground, and often with significant impacts to the landscape.

While there is limited available data on wildlife impacts associated with coal fired power plant operations, one study ${ }^{248}$ estimates that they cause roughly the same numbers of avian mortalities compared to wind turbines, per GWh generated. On a life cycle basis, coal-fired power can cause avian deaths during mining, through collision and electrocution with power plant equipment, and from poisoning and death caused by acid rain and mercury pollution. When projected impacts from climate change are also accounted for, avian mortalities attributed to coal fired power were estimated to be far greater than those attributed to other electric generation technologies.

\section{Natural Gas:}

The land use footprint of a typical $555 \mathrm{MW}$ natural gas combined cycle power plant is estimated to be 20 acres, while a typical $360 \mathrm{MW}$ gas turbine simple cycle plant is estimated occupy roughly half as much land area. When the natural gas plants have equipment for carbon capture on site, then the land use requirements are estimated to increase by 10 percent. ${ }^{249}$ Upstream, the direct land use requirements - and potential ecological impacts - from natural gas production, transmission and storage are more than an order of magnitude greater than the footprint of natural gas power plants. ${ }^{250}$

\section{Nuclear:}

The land use requirements of nuclear power is larger than the footprint of the power station because each plant is required to have an exclusion area and a barrier space that is reserved in case of an accident. ${ }^{251}$ The Nuclear Energy Institute estimates that a typical 1,000 MW nuclear plant requires $1.3 \mathrm{mi}^{2}$, or 830 acres, of land. ${ }^{252}$ While there is no permanent storage for nuclear wastes in the United States, additional land may be required in the future to dispose of spent 
fuel. ${ }^{253}$ The Solid Waste baseline volume ${ }^{254}$ includes more detailed description of solid waste management issues at nuclear facilities.

Similar to coal, upstream mining impacts associated with nuclear energy can be substantial. Potential environmental damages that result from uranium mining includes water contamination from acid mine drainage release and heavy metal exposure to the surrounding ecosystems. On a life cycle basis, avian mortalities from nuclear power are attributed to exposure to toxic waste ponds at uranium mining and milling facilities and collisions with nuclear cooling towers and equipment. ${ }^{255}$

\section{Wind:}

The land required for wind power plants varies with turbine spacing and facility configuration; however, the U.S. Department of Energy estimates that $0.33 \mathrm{~km}^{2} / \mathrm{MW}(82.4$ acres/MW) of land area is typically required for the facility boundary for both land-based and offshore wind development. 256

However, it is important to recognize that wind turbines only use $1-10 \%$ of the wind farm areas, depending on the resource availability and terrain, and the remaining lands are typically still available for grazing, agriculture, ranching, and recreation. ${ }^{257}$ Therefore, the land use requirement for a wind plant is $0.003 \mathrm{~km}^{2} / \mathrm{MW}(0.74$ acres/MW), which includes permanent structures such as access roads, tower foundation pads, and transformer pads. Permanent and temporary direct impact areas on average are $0.3 \pm 0.3$ hectares/MW and $0.7 \pm 0.6$ hectares/MW of capacity, respectively; thus, a total direct impact area is roughly about $1 \pm 0.7$ hectare/MW. ${ }^{258}$ DOE estimates that under a high wind power deployment scenario by 2050 , the total land area affected by wind power installations would be less than $1.5 \%$ of the land area of the United States, with majority (97\%) of that land area remaining available for multiple purposes. ${ }^{259}$

Ecological concerns associated with wind development focus on impacts to avian and bat populations. One primary concern is the direct mortality of birds and bats from collisions; however, indirect impacts can include habitat fragmentation and land degradation. Additional ecological considerations include offshore impacts on marine life and fisheries, and impacts from associated infrastructure (e.g., roads, transmission lines, substations). Noise, visual impacts (from blinking lights and from wind turbines themselves), and property values are all concerns raised by communities with wind developments.

Several studies evaluating wildlife impacts from wind power have been initiated in the United States over the past several years by agencies and organizations such as the U.S. Fish and Wildlife Service Wind Turbine Advisory Committee, the Bats and Wind Energy Cooperative, the American Wind Wildlife Institute, the American Wind Energy Association (AWEA), and the National Wind Coordinating Collaborative (NWCC). Still, the impacts of wind power on wildlife is 
somewhat of an open question as it is difficult to estimate the relationship between preconstruction and post-construction impacts especially related to bird and bat collisions. ${ }^{260}$

The DOE Wind Vision report ${ }^{261}$ finds that bird mortalities due to wind turbines $(0.2$ million birds/year) are much lower compared to bird mortalities from collisions with other engineered structures. For example, estimated total bird deaths caused by power lines and buildings range from 130 up to 1,000, respectively. ${ }^{262}$ Bat mortalities due to wind farms vary within and among regions, for example, two wind plants in the eastern United States have reported averages of up to 30 bat fatalities/MW/yr, but other plants in the East have reported one to two bats/MW/yr. ${ }^{263}$

Other than environmental impacts, some studies have examined aesthetic aspects of wind power, including turbine noise and visibility of wind plants, though these issues have not been found to directly affect human health. ${ }^{264} 265$ Shadow flicker resulting from rotating blades of wind turbines include anecdotal reports of nausea and vertigo, however a study conducted by the United Kingdom (U.K.) Department of Energy and Climate change concluded this does not cause a significant risk to health. ${ }^{266} \mathrm{~A}$ study was conducted by the Lawrence Berkeley National Laboratory (LBNL) to evaluate potential impacts of wind deployments on property value; the report found no statistical evidence of effect on property value due to views in the vicinity of wind turbines after they were constructed. ${ }^{267} 268$

In many cases, public acceptance and environmental concerns for wind plants may be addressed through education and careful siting which will facilitate public involvement and understanding of best practices for wind installations. ${ }^{269}$

Solar Photovoltaic (PV) and Concentrated Solar Power (CSP):

One concern regarding large-scale deployment of solar energy is its potentially significant land use associated with utility-scale ground-mounted PV and CSP facilities. Table 3.2 presents total and direct land-use results for various solar technologies and system configurations, on both a capacity and an electricity-generation basis.

Total area corresponds to all land enclosed by the site boundary and is usually specified in blueprint drawings, typically fenced or protected. Direct impact area comprises land directly occupied by solar arrays, access roads, substations, service buildings, and other infrastructure. The direct-impact area is smaller than the total area and is contained within the total-area boundaries. ${ }^{270}$ The average total area requirements for a PV plant between 1 and 20 megawatt capacity is 8.3 acres per MW. For larger PV plants, the total area needed is 7.9 acres per MW, while CSP plants on average use 10 acres per MW. When weighted by generation rather than capacity, the larger PV plants (3.4 acres per gigawatt-hour per year) and CSP plants (3.5 
acres/GWh/year) use less space than smaller PV plants (4.1 acres/GWh/year), as shown in Table 3.2.

Table 3.2. Summary of Land Use Requirements for PV and CSP Projects in the United States ${ }^{271}$

\begin{tabular}{|c|c|c|c|c|}
\hline \multirow[t]{2}{*}{ Technology } & \multicolumn{2}{|c|}{ Direct Area } & \multicolumn{2}{|c|}{ Total Area } \\
\hline & $\begin{array}{c}\text { Capacity- } \\
\text { weighted } \\
\text { average land } \\
\text { use } \\
\text { (acres/MW } / \mathrm{MW}_{\mathrm{AC}} \text { ) }\end{array}$ & $\begin{array}{c}\text { Generation- } \\
\text { weighted average } \\
\text { land use } \\
\text { (acres/GWh/yr) }\end{array}$ & $\begin{array}{c}\text { Capacity- } \\
\text { weighted } \\
\text { average land } \\
\text { use } \\
\left.\text { (acres/MW } / \mathrm{MW}_{\mathrm{AC}}\right)\end{array}$ & $\begin{array}{c}\text { Generation- } \\
\text { weighted } \\
\text { average land } \\
\text { use } \\
\text { (acres/GWh/yr) }\end{array}$ \\
\hline $\begin{array}{l}\text { Small PV (>1 MW, <20 } \\
\mathrm{MW})\end{array}$ & 5.9 & 3.1 & 8.3 & 4.1 \\
\hline Fixed & 5.5 & 3.2 & 7.6 & 4.4 \\
\hline 1-axis & 6.3 & 2.9 & 8.7 & 3.8 \\
\hline 2-axis flat panel & 9.4 & 4.1 & 13 & 5.5 \\
\hline 2-axis CPV & 6.9 & 2.3 & 9.1 & 3.1 \\
\hline Large PV (>20 MW) & 7.2 & 3.1 & 7.9 & 3.4 \\
\hline Fixed & 5.8 & 2.8 & 7.5 & 3.7 \\
\hline 1-axis & 9.0 & 3.5 & 8.3 & 3.3 \\
\hline 2-axis CPV & 6.1 & 2.0 & 8.1 & 2.8 \\
\hline CSP & 7.7 & 2.7 & 10 & 3.5 \\
\hline Parabolic trough & 6.2 & 2.5 & 9.5 & 3.9 \\
\hline Tower & 8.9 & 2.8 & 10 & 3.2 \\
\hline Dish Stirling & 2.8 & 1.5 & 10 & 5.3 \\
\hline Linear Fresnel & 2.0 & 1.7 & 4.7 & 4.0 \\
\hline
\end{tabular}

Small single-axis designs use $14 \%$ more land than fixed-tilt designs on a capacity-weighted basis but take up $14 \%$ less land on a generation basis due to the increased capacity factor of singleaxis designs. For example, single-axis tracking systems can increase PV generation 12\%-25\% relative to fixed-tilt systems, and double-axis tracking systems can increase PV generation by $30 \%-45 \%$.

Note that a significant amount of PV deployment occurs on rooftops, building facades, and other "zero impact" areas, such as parking lots; however, deployment of PV on these areas can 
have issues with shading and orientation that reduce generation capacity. If PV is deployed in land-based areas, their impact on land can be minimized through the use of brownfield sites or other compromised land, and certain airport land. The land used for deploying ground based solar PVs can sometimes also be used for other purposes like grazing, growing shade tolerant crops, etc., which can help to minimize its impact.

A 2015 MIT report estimated that if all projected U.S. electricity demand were met by PV in the year $2050,{ }^{\text {pp }}$ this would require roughly $33,000 \mathrm{~km}^{2}$ or $0.4 \%$ of U.S. land area. ${ }^{272}$ This land area is roughly equal to the area used by surface mining for coal and is less than the land area occupied by major roads. The currently existing rooftop area within the United States can meet approximately $60 \%$ of the nations projected 2050 electricity needs with PV. ${ }^{273}$ Similarly, NREL estimated that the technical potential exists for rooftop PV to generate 1,432 TWh of electricity, or $39 \%$ of total annual electricity sales. ${ }^{274}$

There have been concerns of bird death with concentrated solar power as birds can fly through the heliostat field and be burned. For example, the Ivanpah solar plant-a 392 MW CSP facility in the Mojave Desert in California-has been the subject of several estimates and measurements of bird deaths ${ }^{275}$. A report by the U.S. Fish and Wildlife Service (USFWS) stated that 47 of the 141 dead birds collected at the Ivanpah CSP plant in October 2013 were killed by the solar flux 276 . In April 2015, "biologists working for the state estimated that 3,500 birds died at Ivanpah in the span of a year, many of them burned alive while flying through a part of the solar installment where air temperatures can reach 1,000 degrees Fahrenheit $\left[540{ }^{\circ} \mathrm{C}\right] "$, reported the Wall Street Journal. ${ }^{277}$ However, the impact to bird populations is relatively minor compared to other factors. For example, research conducted by the U.S. Fish and Wildlife Service found that power lines alone might kill up to 175 million birds annually. ${ }^{278}$ For comparison, free-ranging domestic cats kill $1.3-4.0$ billion birds annually. ${ }^{279}$ Wildlife impact mitigation strategies are discussed below.

\section{Geothermal Energy:}

Geothermal power plants typically use 1-8 acres per MW. ${ }^{280}$ However, the land use impact of a typical geothermal well varies by the capacity, the arrangement of wells and piping systems, and the substation and auxiliary building needs. Advancements in drilling technology have minimized the impact. Modern drilling technology allows several wells to be drilled from one location, which reduces the amount of land needed for drilling pads, access roads, and geothermal fluid piping. 281

pp Assuming that every kWh of energy produced by solar generators can be fully utilized to meet demand regardless of when it is generated. 
Land subsidence can occur from the withdrawal of large amounts of fluid like water, oil, and geothermal fluid from beneath the earth's surface. When large amounts of geothermal fluids are withdrawn and injected below the earth's surface, induced seismicity or earthquake activity are a concern. ${ }^{282}$ This induced seismicity is typically less than 2.5 on the Richter scale (earthquakes usually are not felt below 3.5). ${ }^{283}$ Recently, some areas near The Geysers geothermal field in California have been experiencing increased seismic activity. There is concern that it could be due to wastewater injection, which helps replenish the geothermal resource, from the Lake County Sanitation District. DOE, the U.S. Geologic Survey, and the geothermal power companies at The Geysers are monitoring this activity. ${ }^{284}$

The land use footprint of a typical geothermal power plant can also be used for livestock grazing or other agricultural purposes. For example, the geothermal plant at Mammoth Lakes, California, is used for outdoor activities. Geothermal plants can also minimize any impact on scenic and recreational area, if sited properly. ${ }^{285}$

Hot water pumped from underground reservoirs contains high levels of sulfur, salt, and other minerals, leading to concerns about potential contamination of underground sources of drinking water. However, no cases of water contamination from geothermal sites have been reported in the United States. ${ }^{286}$

\section{Conventional hydroelectric power:}

Land use requirements for conventional hydroelectric power vary significantly, depending on site-specific conditions, from 2,350 to $25,000 \mathrm{~m}^{2} / \mathrm{GWh} .{ }^{287}$ The scale of land requirements of associated water reservoir depends on the steepness of terrain and the height of the dam. Run of river plants, which do not create water reservoirs, require far less land area, by orders of magnitude. For example, Table 3.1 illustrates that the land use factor $\left(\mathrm{MW} / \mathrm{km}^{2}\right)$ for run of river hydro is a small fraction of land use factors for other renewable energy technology types.

Ecosystem impacts caused by hydroelectric power plants depend on the size and flow rate of the dammed river; the climate and habitat conditions; type, size, design and operation of the plant; and if cumulative impacts occur because the plant is located upstream or downstream of other projects located on the same river. ${ }^{288}$ Flooding land for a hydroelectric reservoir can also have significant environmental impacts through the inundation of forests, wildlife habitat, agricultural land, and scenic lands. ${ }^{289}$ Hydroelectric power can also have significant impacts on aquatic ecosystems such as fish and other organisms, which can be injured and killed by turbine blades. Reservoir water is usually more stagnant than normal river water, which can lead to algae blooms and other aquatic weeds crowding out native aquatic life. This is sometimes controlled through manual harvesting or by introducing fish that eat non-native species of plants and animals. In hydropower plants, if water in a dammed reservoir is not released appropriately, water levels downstream will drop and animal and plant life can be harmed. ${ }^{290}$ 
Also, operating a hydroelectric power plant may change the water temperature (the slower moving water absorbs more energy from the sun) and the river's flow and thereby may harm native plants and animals in the river and on land. ${ }^{291}$ Colder water sinks to the bottom because of its higher density, leading to stratification of temperature. The colder water at the bottom of reservoirs contains higher levels of dissolved oxygen which impacts downstream habitat conditions. Supersaturated water can enter tissues of fish and other species. If fish swim from a supersaturated area to a lower pressure area, this may cause injury or even death to fish. ${ }^{292}$

Most dams are used for other purposes such as irrigation, flood control, and water treatment. For example, dams on the Columbia and Tennessee Rivers are used for irrigation, flood control, transportation, recreation and production of electricity.

\subsection{LAND USE AND ECOLOGICAL IMPACTS OF TRANSMISSION AND DISTRIBUTION}

Although the environmental impacts of electricity transmission and distribution (T\&D) appear to be much smaller than the environmental impacts of electricity generation, they are not negligible. ${ }^{293}$ Perhaps because they do not provide large point sources of pollution and are geographically more expansive, the impacts of the T\&D system are also not well characterized. ${ }^{294}$ Electrical grid systems deliver electricity from numerous and diverse generating units to hundreds of millions of consumers via high- and low-voltage power lines and cables, towers and poles, transformers and substations, and a variety of electrical equipment required to maintain grid reliability and power quality.

Altogether, the U.S. grid consists of more than 200,000 miles of high-voltage transmission lines directly connected to power plants delivering $A C$ electricity with the same relative frequency to a large number of local distribution companies. An additional six million miles of distribution lines and about 55,000 substations bring the power directly to about 300 million customers. ${ }^{295}$

296 With the need to reduce greenhouse gas emissions, meet rising demands for reliable power, and accommodate new technologies and services, grid infrastructure investments are expected to continue to be substantial well into the future. Thus, it is timely to consider ways that T\&D land use and ecological impacts can be minimized.

T\&D systems have a wide array of direct and indirect environmental impacts. These can be divided by life cycle stage, distinguishing between the impacts that occur when T\&D assets are being constructed, and those that occur during the operation of the electric grid. There are also opportunities for the construction and operation of grid infrastructure to help enable better environmental outcomes; for example, transmission lines are necessary to connect remotely located lower-emitting generation sources to load centers. Additionally, clearings for 
transmission lines also help create firebreaks, which can reduce the impacts of wild fires and improve emergency access.

Siting and Construction Phase: The routing of overhead power lines can often be optimized to reduce environmental impacts. ${ }^{297}$ It may be possible to reduce potential environmental impacts by adjusting the proposed route, choosing a different type of pole structure, or using different construction methods. For example, during design phase by using corridor-sharing route it is possible to minimize right-of-way requirements. Choosing a different transmission pole with different construction requirements and aesthetic appeal can be considered during design phase. Minor adjustments in pole locations can be done to avoid archeological sites or minimize effects on agriculture operations. Construction of power lines when the ground is frozen and vegetation is dormant can be considered to minimize impacts to wetland habitat; delaying construction in agricultural areas until after harvest can help minimize crop damage. Clearings for transmission lines also create meadows and edge habitats that can be managed in ways that are beneficial for certain species. ${ }^{298}$

Putting power lines underground can address some of the adverse land use and ecological impacts of overhead lines. It is common to have underground low-voltage distribution lines in residential areas; burying transmission lines underground, on the other hand, is uncommon because it very expensive (2-10 times more than building an overhead line). ${ }^{299}$ The Public Service Commission of Wisconsin reported the cost of a $69 \mathrm{kV}$ overhead single-circuit transmission was about $\$ 285,000$ per mile. ${ }^{300}$ Conversely, a $69 \mathrm{kV}$ underground line (without terminals) costs about $\$ 1.5$ million per mile. ${ }^{301}$

Expanding T\&D capacities along existing rights-of-way-rather than creating a new right-ofway-will generally minimize damage to land use functionality, aesthetics, natural habitats, and ecological systems. However, using an existing T\&D right-of-way may not be the best choice especially when new residential areas have been built around the existing line; when electricity use has increased more in other areas, and when a wider right-of-way is needed because of the size of the new line.

Public opposition to high-voltage transmission line construction is common, particularly where trust in institutions is low. ${ }^{302}$ Local opposition can make transmission lines difficult to site as lengthy negotiations can contribute to project delays or ultimately prevent projects from being built. ${ }^{303}$ As a result of public opposition, transmission permitting, siting and construction can take 7-10 years longer than permitting, siting, and construction of generation facilities. ${ }^{304}$ Siting a high-voltage transmission line often involves media attention, contentious municipal hearings, and legislative and litigated attempts to stop the project; this process can be time consuming and costly. When power lines, their access roads, and associated equipment are placed in undeveloped areas on new corridors, the environmental impacts can be more 
substantial, including the disturbance of forests, wetlands, and other natural areas and associated viewsheds. These disturbances may be temporary or permanent, potentially establishing vehicle access into areas that had been road-less. Routing infrastructure through undeveloped areas also comes with some uncertainty, since sensitive cultural artifacts or endangered species are currently not mapped comprehensively.

T\&D infrastructure requirements for distributed generation (DG) systems including microgrids have smaller land use footprints because DG units are located closer to end users, which can reduce the need for new or expanded transmission lines. However, such distributed generation systems could require an expansion of local transformer and substation capacities (the average cost of updating a substation is $\$ 40 / \mathrm{kVA}$ ) and could be associated with greater investments in distributed storage and other equipment that might pose aesthetic issues because of their proximity to population centers. ${ }^{305}$ DG can be added to the grid in small capacities, allowing generation to closely follow the geography of demand. DG units located close to load centers can defer T\&D system investment and help utilities minimize electric power purchases during high price periods. However, the feasibility of a DG alternative to a T\&D alternative requires a detailed engineering evaluation on factors like electricity price, fuel price, combination of existing capacity and load growth rate, and hours of operation. ${ }^{306}$ For example, a $1 \mathrm{MW}$ natural gas fired DG unit was installed at a school, thereby deferring a \$3.8 million substation expansion project for five years. ${ }^{307}$

Driven by state-level renewable portfolio standards and federal polices, renewable generation is expected to increase. As a result, new transmission lines are being proposed to connect renewable resources located far from load centers to the existing grid. ${ }^{308}$

Operational Phase: During operation, a principal concern is that transmission and distribution lines are responsible for an efficiency loss of roughly $6 \%$ of total U.S. electricity generation. ${ }^{309}$ Such losses require that more electricity be generated from power plants, which may result in additional air and water pollution from power generation facilities. ${ }^{310}$

Avian mortalities from collisions with transmission lines are also an environmental cost of the T\&D system. Electricity infrastructure like towers, poles, stacks, and transformers put birds at risk from collisions with wires and towers, and birds and other wildlife can be subject to electrocution. This can result in outages and also reduce bird and other wildlife populations. Adverse effects on certain birds (e.g., electrocution of eagles) may result in penalties. ${ }^{311}$ One inventory of bird mortality in Canada estimated that transmission lines across Canada cause the death of 2.5 to 25.6 million birds each year. ${ }^{312}$ For perspective, Canada's transmission system has about half the mileage of the U.S. system. Bird collisions vary by habitat type, species size, and scavenging rates, and they appear to be higher during migration. ${ }^{313}$ Proactive planning can help reduce these impacts on avian and other wildlife populations. 
Power lines require routine maintenance. Utility companies must manage vegetation in powerline right of ways (ROWs) on Federal lands to prevent power outages, wildfires, and the spread of invasive species and to protect human health, property, and natural and cultural resources. Trees and other plants near the lines have to be trimmed to keep them from touching the lines and potentially causing outages; NERC reliability standard FAC-003-2 provides information and guidance on how to improve the reliability of T\&D systems by preventing vegetation related outages. ${ }^{314}$ On some power line corridors herbicides are used to control vegetation, which can pollute ground water and harm wildlife if done improperly. Some electric utilities are demonstrating more of a commitment to wildlife habitat and conservation when managing vegetation in proximity to their transmission lines. ${ }^{315}$ The North American Electric Reliability Corporation (NERC) has Protection and Control (PRC)-005, 008, 011 and 017 standards that require T\&D owners to document maintenance requirements with procedures and records to ensure reliable operation. ${ }^{316}$

In addition to ensuring the reliable delivery of electricity, integrated vegetation management in powerline ROWs can prevent the spread of invasive species, provide habitat for pollinators, and act as an effective firebreak for the control and suppression of wildfire. Coordination between Federal agencies and utility companies before and during ROW establishment and maintenance activities may help to avoid interruptions in electric service while maintaining the environmental and cultural integrity of the lands they occupy. In 2006, the Edison Electric Institute and Federal agencies entered into a memorandum of understanding (currently being updated) to facilitate cooperation and coordination regarding vegetation management within and adjacent to existing and future powerline ROWs. ${ }^{317}$

Additional operation concerns surround the electromagnetic field (EMF) effects from conventional power sources, such as power lines and electrical substations. Findings from research on this topic suggest a possible link between EMF field strength and an increased risk for childhood leukemia as well as the neurobehavioral function of children. However, findings suggest that these associations are weak and dissipate quickly with distance from the EMF sources. 318319

The few studies that have been conducted on adults show no evidence of a link between EMF exposure and adult cancers, such as leukemia, brain cancer, and breast cancer. Nevertheless, the National Institute of Environmental Health Sciences (NIEHS) recommends continued education on practical ways of reducing exposures to EMFs. ${ }^{320}$

\subsection{THE SITING AND PERMITTING OF ELECTRICITY INFRASTRUCTURE}

The transformation of the U.S. electricity system in response to changing market, technology, economic, and policy conditions requires effective siting and permitting capabilities at all levels 
of government, particularly at the state and local level where most siting and permitting decisions are made. Impacts on safety and security, as well as environmental and community resources such as air, water, land and cultural resources need to be minimized, while at the same time delays for project permitting and approval need to be limited. This section describes various aspects of the siting and permitting of electricity infrastructure, focusing on the role of the Federal government and ongoing initiatives to modernize permitting and environmental review processes. Chapter $9^{321}$ of the first installment of the QER includes more detailed discussions of the multiple siting, permitting, and review authorities, the role of governments at a variety of levels, and the public engagement process. The Jurisdictions Baseline is another valuable resource for consideration of these issues.

Planning and permitting new transmission infrastructure is uniquely challenging. Managing land use and minimizing environmental impacts for interstate infrastructure networks inherently involves working across jurisdictions and with a wide range of stakeholders. Since there can be multiple federal agencies involved with permitting interstate transmission, the Obama Administration has taken several steps to help modernize the Federal government's role in the permitting and review processes. ${ }^{322}$ Another critical need is for active and effective coordination between federal, state and local governments, to help enable well-informed decision making that strikes a fair balance between a broad range of public and private interests.

\subsubsection{State Authorities for Infrastructure Siting}

With limited exception, states have primary authority over siting, permitting, and review of electric transmission projects. Each state has different procedures to follow for approving a transmission line and interstate lines must comply with the legal requirements of each state. ${ }^{323}$

The majority of transmission projects receive approval from the state where they apply for residence. To apply for a permit, the project sponsor is required to file an application with the state. ${ }^{324}$ Additionally, each state employs a different approval procedure. Certain states have a central siting authority that has the sole jurisdiction of permitting for the state, while others have splintered regulatory authority. Consideration for local needs versus regional needs also differ in permitting decisions depending on the state. ${ }^{325}$

There are a few cases in which federal agencies will review applications to states. EPAct 2005 grants FERC the right to review an application for permitting if approval is withheld by a state for more than a year, cannot site a transmission facility, or is unable to consider interstate benefits of a project in the national corridor. ${ }^{326}$ Key siting decisions are made by federal agencies, particularly in states with large acreages of federal land located near generation sources or along established energy transmission corridors. All projects that are proposed on 
federal lands, affect regulated air or water quality, or receive federal funding require a federal permitting review. ${ }^{327}$

The federal system of the United States creates overlapping jurisdiction between federal, state, local, and tribal governments. For instance transmission lines that cross state borders must be in accordance with all interstate laws and a site permit must be obtained from each state. ${ }^{328} 329$ Local governments may also adopt zoning requirements that differ from the state regulations or even the regulations of neighboring communities. ${ }^{330}$ Tribal governments may also become a participant in the permitting decision if a project may disrupt cultural or historic properties or resources. ${ }^{331}$

Thus communication and coordination between the various levels of government are imperative to efficiently review a permitting application. ${ }^{332}$ Partnerships between federal and state actors and unifying agencies with different missions have previously expedited the permitting review process. Examples include the Transmission Siting Task Force developed by the Western Governors' Association (WGA) and state siting agencies and the partnership of the Pacific Northwest Regional Infrastructure Team and Renewable Energy Action Team in California. Another partnership between DOE and WGA yielded the Regulatory and Permitting Information Desktop Toolkit. This provides actors with publicly available information in one easy-to-access resource. Further cooperation occurred when The Council of State Governments composed an Interstate Transmission Line Siting Compact. The compact provides model language for state legislatures to adopt interstate cooperation. ${ }^{333}$

\subsubsection{Federal Authorities for Infrastructure Siting}

While state and local governments have the primary responsibility and authority to permit the siting of new energy infrastructure, the Federal government plays an important role in a number of ways. As discussed in Chapter 9 of the first installment of the QER, any federal action (such as co-funding a renewable energy generation project) requires the responsible federal official to consider the potential environmental impacts of the proposed action and any reasonable alternatives. ${ }^{334}$ This requirement is specified in the National Environmental Policy Act (NEPA) of 1969 and the Council on Environmental Quality Regulations for Implementing the Procedural Provisions of NEPA.

Chapter 7 of the first installment of the QER ${ }^{335}$ described typical impacts that are analyzed as part of the environmental review processes for energy transmission and distribution infrastructure siting. These impacts involve many cross-cutting issues, some of which are environmental while others are more cultural and social in nature (Appendix F). NEPA establishes a framework for assessing these impacts during the planning process before a 
Federal agency decides whether to fund, conduct, permit, or otherwise approve a proposed infrastructure project. ${ }^{\text {qq }}$

NEPA stipulates that one of three levels of review is conducted by federal agencies: a categorical exclusion, environmental assessment, or environmental impact statement. The level of review depends upon the scope and complexity of the project and the intensity of potential impacts on communities and the environment. Such reviews can take time to address community and environmental impacts. ${ }^{336}$ Affected residential communities, nonprofit organizations, and other stakeholders also participate in the NEPA process through public comment opportunities. ${ }^{337}$ Depending on the level of NEPA review required for any particular project, public participation and engagement with diverse sets of stakeholders are essential to reduce the risks associated with uncertainty and potential challenges. Early and robust public engagement is a recognized best practice and can reduce delays and improve projects. 338339 For example, public meetings, mailings, and website information have been recommended by stakeholder groups. The Western Governors Association recommends the use of "Live GIS" at public meetings to illustrate proposals using geographic data including project boundaries, the location of access roads, the habitats of protected wildlife, and property boundaries. By providing customized information on touch screen computer monitors, the concerns of individuals can be specifically clarified. ${ }^{340}$

\subsubsection{Mitigation of Environmental Impacts}

In their analyses, permitting agencies typicallyrr consider mitigation requirements that may be imposed as conditions for addressing unavoidable environmental harms. Decades of experience with siting energy transmission and distribution infrastructure have resulted in the development of various measures and methods for offsetting impacts to affected communities and ecosystems, including avoidance, minimization, and compensation. A brief summary of these are described in the first installment of the QER and are reproduced here, in Box 1.

\footnotetext{
qq Under NEPA and the Council on Environmental Quality Regulations Implementing the Procedural Provisions of the National Environmental Policy Act, any T\&D infrastructure proposal requiring a Federal agency to take an action (such as funding, permitting, or otherwise approving a pipeline or electricity transmission project) requires preparation of an environmental review that considers the environmental impact of the proposed action and any reasonable alternatives prior to the agency proposed action (42 U.S.C. §§ 4321-4347 and 40 C.F.R. §§ 1500-1508). ${ }^{r r}$ Agencies must consider mitigation when completing an Environmental Impact Statement (EIS) and mitigation is often considered when completing an environmental Assessment (EA).
} 


\section{Box 1: Mitigation Options to Address Adverse Environmental Options ${ }^{341}$}

Mitigation is an important mechanism for agencies to use to avoid, minimize, rectify, reduce, or compensate the adverse environmental impacts associated with their actions. ${ }^{\text {ss }}$ Federal agencies typically rely upon mitigation to reduce environmental impacts through modification of proposed actions and consideration and development of mitigation alternatives during the NEPA process. ${ }^{\text {tt }}$

Resource-specific mitigation measures can be applied to avoid or minimize impacts from an electricity infrastructure project. In order to identify and implement appropriate mitigation measures, first the potential impacts of a project on a specific resource must be assessed. Then, project-specific and site-specific factors must be evaluated to determine whether the impact can be avoided or mitigated, what action can be taken, how effective the mitigation measure will be, and the cost effectiveness of the measure.

Mitigation is of particular importance to Federal agencies that manage hundreds of millions of acres of public lands, which comes with a responsibility to sustain a vast array of resources, values, and functions. For example, public lands contain important wildlife habitat and vegetative communities -in addition to recreational opportunities and ecosystem services, cultural resources, and special status species. These lands are managed for the use and enjoyment of present and future generations. The location, construction, and maintenance of energy infrastructure should avoid, minimize, and, in some cases, compensate for impacts to these public resources, values, and functions. Mitigation is also of critical importance to those agencies responsible for protecting the nation's waters. Applying this mitigation hierarchy early in the planning for TS\&D infrastructure will help provide better outcomes for the impacted resources, values, and functions.

\subsubsection{Federal Initiatives to Modernize Permitting and Review Processes}

In addition to fulfilling NEPA requirements, Federal government agencies have valuable roles to play in supporting more efficiency and transparency during permitting and review processes while enabling better outcomes for public health, the environment, safety and communities. To help address the myriad challenges of obtaining permits and approvals, President Obama has issued a number of directives for Federal agencies to take action, as outlined in a July, 2015 White House Report: ${ }^{342}$

- In October of 2009, nine Federal agencies signed a memorandum of understanding with the goal of reducing approval time and reduce barriers to siting new transmission lines.

- On Aug. 31, 2011, a Presidential Memorandum called for expedited review of "priority projects" and improved accountability, transparency, and efficiency.

- Executive Order 13604, issued on March 22, 2012, directed federal agencies to improve the efficiency and transparency of permitting and review processes for infrastructure projects while producing measurably better outcomes for communities and the environment.

\footnotetext{
ss 40 C.F.R. $\S 1508.20$.

tt The Council on Environmental Quality's NEPA regulations require agencies to identify in their Record of Decision any mitigation measures that are necessary to minimize environmental harm from the alternative selected (40 C.F.R. $\S 1505.2(c))$. The NEPA analysis can also consider mitigation as an integral element in the design of the proposed action. The regulations further state that a monitoring and enforcement program shall be adopted where applicable for any mitigation (40 C.F.R. $\S 1505.2(c)$ ).
} 
- On May 17, 2013, the President released a memorandum directing an interagency steering committee to develop a plan for institutionalizing best practices for permitting and review into standard practice for all major infrastructure projects.

- On June 7, 2013, the President issued a memorandum directing Federal agencies to "develop an integrated, interagency pre-application process for significant onshore electric transmission projects requiring Federal approval."

- December 5, 2015, the FAST Act became law, including Title 41, which establishes a Federal Infrastructure Steering Council to inventory major infrastructure projects that are subject to NEPA and improve the review process through "greater agency accountability, efficiency and increased transparency."

Additionally, the federal government is undertaking several actions to reduce the aggregate permitting and review time for infrastructure projects, while improving environmental and community outcomes. This includes a number of Federal and regional initiatives (outlined in Table 3.3) that are designed to support better decision making in the following ways:

1) Facilitate better coordination between permitting authorities at all levels of government,

2) Develop and publish relevant information, data and tools,

3) Support infrastructure planning; establish rights of way for energy projects, and

4) Conduct technology research and development. 
Table 3.3. Federal and Sub-National Initiatives to Modernize Electric Infrastructure Permitting and Review Processes. ${ }^{343}$

Initiative Title Description (Scope and Specific Focus Areas)

\begin{tabular}{|c|c|}
\hline \multicolumn{2}{|c|}{ Facilitate better coordination between permitting authorities, increase transparency } \\
\hline $\begin{array}{l}\text { Establishing an Implementation Plan to Modernize } \\
\text { Permitting }\end{array}$ & $\begin{array}{l}\text { National, Federal plan includes four strategies, } 15 \\
\text { reforms, and nearly } 100 \text { near-term and long-term } \\
\text { milestones. }\end{array}$ \\
\hline Creating a Permitting Dashboard & $\begin{array}{l}\text { National, web-based dashboard to track permit } \\
\text { process for Federally designated projects }\end{array}$ \\
\hline $\begin{array}{l}\text { Developing an Interagency Rapid Response Team for } \\
\text { Transmission }\end{array}$ & $\begin{array}{l}\text { National, improve Federal interagency coordination, } \\
\text { Tribal consultation and conflict resolution for } \\
\text { challenging transmission projects }\end{array}$ \\
\hline $\begin{array}{l}\text { The Western Governors Association Regulatory and } \\
\text { Permitting Information Desktop (RAPID) Toolkit }\end{array}$ & $\begin{array}{l}\text { Western U.S., includes wiki platform for stakeholder } \\
\text { and agency collaboration }\end{array}$ \\
\hline \multicolumn{2}{|c|}{ Publish information, data and tools } \\
\hline The Environmental Protection Agency's NEPAssist & National, web-based mapping tool \\
\hline $\begin{array}{l}\text { Fish and Wildlife Service - Information, Planning, and } \\
\text { Conservation Tool }\end{array}$ & $\begin{array}{l}\text { National, help identify endangered and threatened } \\
\text { species before } \\
\text { beginning project design }\end{array}$ \\
\hline Army Corps' Federal Support Toolbox & $\begin{array}{l}\text { National, "one-stop-shop" online water resources data } \\
\text { portal }\end{array}$ \\
\hline $\begin{array}{l}\text { Eastern Interconnection States Planning Council } \\
\text { Energy Zones Mapping Tool }\end{array}$ & $\begin{array}{l}\text { Eastern U.S., includes } 273 \text { GIS data layers and links to } \\
\text { key resources. }\end{array}$ \\
\hline $\begin{array}{l}\text { Energy Zones Mapping Tool (EZMT) for the Eastern } \\
\text { Interconnection Planning Collaborative }\end{array}$ & $\begin{array}{l}\text { Eastern U.S., mapping clean energy resources and } \\
\text { transmission }\end{array}$ \\
\hline \multicolumn{2}{|c|}{ Support infrastructure planning } \\
\hline $\begin{array}{l}\text { Undertaking landscape- and watershed-level } \\
\text { mitigation and conservation planning }\end{array}$ & $\begin{array}{l}\text { National, environmental mitigation and resource } \\
\text { protection at the landscape- and watershed-level }\end{array}$ \\
\hline $\begin{array}{l}\text { Designating corridors for pipelines, electric } \\
\text { transmission lines, and related infrastructure }\end{array}$ & $\begin{array}{l}\text { Western U.S., establish rights of way to help promote } \\
\text { energy development, resolve resource disputes and } \\
\text { reduce congestion }\end{array}$ \\
\hline \multicolumn{2}{|c|}{ Technology research and development } \\
\hline Promoting grid modernization, DOE & Enhance security capabilities and stakeholder support \\
\hline
\end{tabular}

Furthermore, the first installment of the QER made several recommendations for enhancing the federal government's siting, permitting, and review processes (Box 2). The last of these was adopted by Congress at the end of 2015. 


\section{Box 2. Recommendations from the first installment of the Quadrennial Energy Review ${ }^{344}$}

Allocate resources to key federal agencies involved in the siting, permitting, and review of infrastructure projects. Federal agencies responsible for infrastructure siting, review, and permitting have experienced dramatic appropriations cuts and reductions in staff. Many of the components of the overall effort to improve the federal siting and permitting processes have been stymied in recent years by appropriations shortfalls. Congress should fully fund these priorities.

Prioritize meaningful public engagement through consultation with Indian Tribes, coordination with state and local governments, and facilitation of non-federal partnerships. Early and meaningful public engagement with affected residential communities, nonprofit organizations, and other non-federal stakeholders through the National Environmental Policy Act process and other forums can reduce siting conflicts. Federal agency coordination with state and local governments and government-to-government consultation with affected Indian Tribes should remain a federal Government priority. When possible, federal agencies should co-locate energy infrastructure environmental review and permitting staff from multiple federal agencies' regional and field offices.

Expand landscape- and watershed-level mitigation and conservation planning. When adverse impacts to the nation's landscape cannot be avoided or minimized any further, federal agencies should seek innovative approaches to compensate for adverse project impacts commensurate with the scope and scale of the project and effects to resources. Through mitigation planning at a landscape, ecosystem, or watershed scale, agencies can locate mitigation activities in the most ecologically important areas.

Adopt Administration proposals to authorize recovery of costs for review of project applications. Consistent with the proposal in the President's Fiscal Year 2016 Budget request, additional flexibility for certain agencies to accept funds from applicants would be appropriate and could expedite the Federal permitting and review process.

Enact statutory authorities to improve coordination across agencies. Congress should authorize and fund the Interagency Infrastructure Permitting Improvement Center in the Department of Transportation, as set forth in Section 1009 of the Administration's draft legislation for the Generating Renewal, Opportunity, and Work with Accelerated Mobility, Efficiency, and Rebuilding of Infrastructure and Communities throughout America Act, or GROW AMERICA Act.

\subsection{OPPORTUNITIES TO FURTHER REDUCE LAND USE AND ECOSYSTEM IMPACTS}

Improving environmental outcomes from infrastructure siting can be accomplished in a number of ways and through the efforts of agencies at all levels of government and also by the private sector.

Multiple uses for rights of way, repowering and repurposing degraded lands or brownfields: Instead of using pristine lands, electricity infrastructure can be sited at less environmentally sensitive locations such as Superfund sites, brownfields, landfills, abandoned mining land, or existing transportation and transmission corridors. Through its cataloging of federal and state tracked contaminated lands, landfills and mine sites, the EPA has identified thousands of potential sites that could potentially ameliorate such impacts. ${ }^{\text {uu }}$

Reducing Impacts to Wildlife:

uu US EPA, RE-Powering America's Land http://www.epa.gov/sites/production/files/2015-

03/documents/repower_technologies_solar.pdf 
With increase in wind power generation, the wind industry and regulation agencies have been working to minimize the impacts of wind plants on migratory birds and other species of concern and their habitat. For example, siting, construction and operations procedures are modified such as use of taller tabular towers, slower rotor machines, etc. The U.S. Fish and Wildlife Service (USFWS) enforces the Bald and Golden Eagle Protection Act (Eagle Act) that provides high protection for bald and golden eagle species. USFWS issued the Land-Based Wind Energy Guidelines in March 2012. ${ }^{345}$ These voluntary guidelines provide a structured scientific process for addressing wildlife conservation concerns at all stages of land-based wind energy development. In addition, USFWS issued the Eagle Conservation Plan Guidance Module 1 Land-based Wind Energy Version 2. ${ }^{346}$ This guidance supplements the Land-Based Wind Energy Guidelines and provides specific in-depth guidance for conserving bald and golden eagles in the course of siting, constructing, and operating wind energy facilities. In 2011, the USFWS introduced Indiana Bat Section 7 and Section 10 Guidance for Wind Energy Projects to act as an interim step needed to get full understanding of Indiana bat wind plant interactions. ${ }^{347}$ There is a need for more data collection that will help wind developers minimize bat mortality.

DOE sponsors research and technology $R \& D$ to reduce the environmental impacts of renewable energy technologies. For example, one DOE project could help to decrease fish deaths caused by hydroelectric power generators to $<2 \%$ from $5-10 \% .{ }^{348}$ Approaches like construction of fish ladders and elevators help fish to move around dams to the spawning ground upstream; for example, in the Columbia River, fish ladders approach is used to help salmon step up the dam to the spawning grounds upstream. Also, since 2003 the Bats and Wind Energy Cooperative (BWEC) has engaged in DOE-funded research assessing the impact of altering the minimum wind speed at which wind turbines begin producing power and the use of ultrasonic acoustic deterrents to help reduce impacts of wind turbines to bats. ${ }^{349}$

In general, ecological impacts can also be reduced by increasing the availability of relevant data to inform decision-making. For example, Tethys was developed in 2009 by the Pacific Northwest National Laboratory (PNNL) to support DOE Technologies Offices by facilitating the exchange of information and data on the environmental effects of wind and marine renewable energy technologies. ${ }^{350}$ Another example is the Wind-Wildlife Impacts Literature Database (WILD), which is a searchable collection of documents that now focuses on the impact on all wildlife from a variety of technologies, including land-based and offshore wind energy, small wind turbines, and marine energy development. ${ }^{351}$

Non-transmission alternatives (NTAs):

FERC's Order No. 1000 requires "comparable consideration of transmission and nontransmission alternatives in the regional transmission planning process." 352 This Order reiterates the principle of comparable consideration, which was included in Order No. 890, and 
stems from the requirement that rates be just and reasonable. In Order No. 1000, FERC recognized that "in appropriate circumstances, alternative technologies may be eligible for treatment as transmission for ratemaking purposes." As an example of this treatment, In Western Grid Development, LLC, FERC held that an energy storage project was eligible for cost allocation as a wholesale transmission project because the manner in which the storage project functioned mimicked traditional transmission infrastructure. ${ }^{353}$ Non-transmission alternatives (NTAs) would appear to be particularly appropriate where transmission infrastructure is expensive, as when it crosses areas of high ecosystem value. ${ }^{354}$

State Public Utilities Commissions (PUCS) are required to follow FERC Order 1000 requirement of "comparable consideration" for transmission and non-transmission alternatives. As a result, the Maine PUC recently approved the Smart Grid Reliability Pilot Project in the Boothbay SubRegion of Central Maine Power Company's electric grid. At a cost of \$6 million, the NTA project avoided an $\$ 18$ million rebuild of a transmission line, saving consumers $\$ 12$ million. The Pilot Project includes five categories of NTAs - efficiency, photovoltaic solar, demand response and peak shifting, back up generation and battery storage - dispersed across about twenty locations in the sub-region. ${ }^{355}$

Distribution-level projects also demonstrate the cost-effectiveness of NTAs. In 2003, Con Ed launched a program to defer distribution system upgrades using a competitive bidding process. Between 2003 and 2010, the company employed geographically-targeted efficiency programs to defer upgrades in more than one third of its distribution networks. In some cases, the efficiency investments not only deferred upgrades, but also bought enough time to allow the utility to refine load forecasts to the point where there are no planned future capacity expansions. $^{356}$

\section{Data and Analytical Needs:}

There is a need for updated life cycle assessment research with consistent methodologies applied to land use requirements - and ecological impacts - to enable more direct comparisons between electricity generation technologies and associated infrastructure. More research and increased availability of data would increase the transparency of environmental impacts to developers, regulators and the public, which, in turn would help inform more effective strategies for mitigating ecological impacts of electricity infrastructure and operations.

Also, while many studies describe the external costs associated with the land-use and ecosystem impacts of electricity generation, few studies attempt to monetize them. ${ }^{357}$ The externalities due to transmission and distribution of power generation are believed to be small compared to other externalities of power generation and are typically not monetized by power generation impact studies. ${ }^{358}$ These are areas in which current data and analytical tools are apparently lacking. 


\section{WATER QUALITY}

Certain types of power generation facilities generate pollution that contributes to water quality concerns. This occurs through a variety of mechanisms. As discussed above, air emissions of mercury, $\mathrm{NO}_{x}$, and $\mathrm{SO}_{2}$ contribute to water pollution through wet deposition. Additionally, steam electric plants may generate wastewater streams that can impact ground water and surface water quality. The plants generate wastewater pollution in the form of chemical pollutants and thermal pollution (heated water) from their water treatment, power cycle, ash handling and air pollution control systems, as well as from coal piles, yard and floor drainage, and other miscellaneous wastes. ${ }^{359}$ The issue of thermal pollution is addressed in more detail in the Energy-Water baseline volume, while management of solid waste is addressed in the Solid Waste baseline volume. ${ }^{360}$

Wastewater pollutants include both toxic and bioaccumulative pollutants such as arsenic, lead, mercury, selenium, chromium, and cadmium. Wastewaters also contain significant levels of chloride, total dissolved solids (TDS), total suspended solids (TSS), and nutrients. Steam electric power plants account for about 30 percent of all toxic pollutants discharged into surface waters by all industrial categories regulated under the Clean Water Act. ${ }^{361}$

Some of the pollutants discharged by the electric power industry can cause severe health and environmental problems in the form of cancer and non-cancer risks in humans, lowered IQ among children, and deformities and reproductive harm in fish and wildlife. Many of these pollutants, once in the environment, remain there for years (i.e., bioaccumulative pollutants). Due to their close proximity to these discharges and relatively high consumption of fish, some minority and low-income communities have greater exposure to, and are therefore at greater risk from, pollutants found in steam electric power plant discharges. ${ }^{362}$

\subsection{SOURCES OF WATER POLLUTION IN THE ELECTRIC POWER INDUSTRY}

Major waste streams from steam electric power plants include wastewaters from wet flue gas desulfurization (FGD) air pollution control systems, discharges associated with coal ash handling operations, wastewaters from flue gas mercury control (FGMC) systems, coal pile runoff, condenser cooling, equipment cleaning, and leachate from landfills and impoundments. ${ }^{363}$ Figure 4.1 displays the major sources of pollution from a representative coal-fired power plant. 
Figure 4.1. Major sources of wastewater from steam electric power plants. ${ }^{364}$

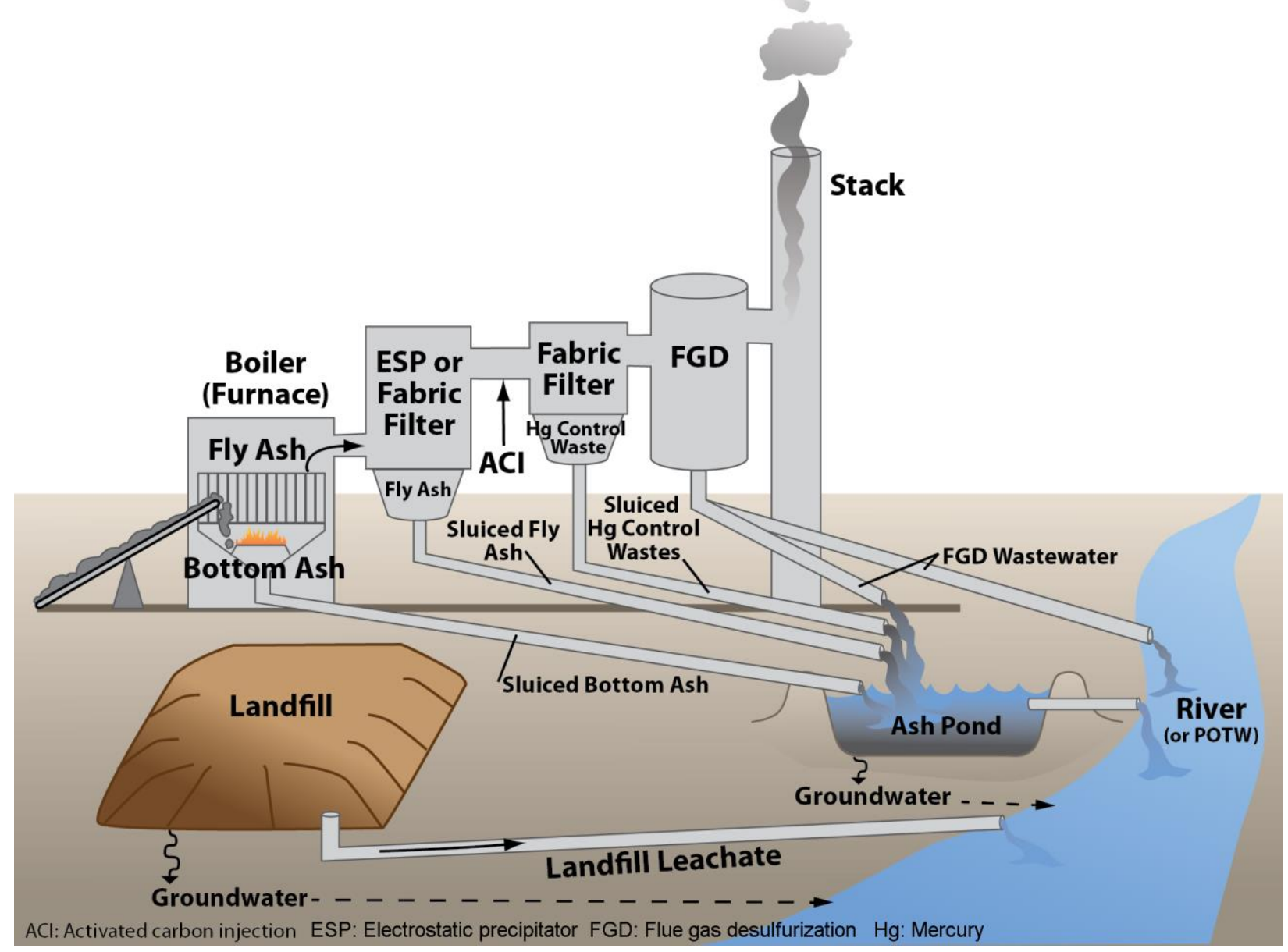

Key wastewater streams from steam electric power plants include wastewater from fly and bottom ash handling, flue gas desulfurization, flue gas mercury control, and landfill leachate.

\section{Wastewater streams:}

FGD scrubber systems are used to remove sulfur dioxide $\left(\mathrm{SO}_{2}\right)$ from stack emissions, and their use has increased substantially in the last couple decades as power plants have taken steps to address federal and state air pollution control requirements. In wet FGD systems, ${ }^{\text {vv }}$ the flue gas comes in contact with a liquid stream containing a sorbent which removes $\mathrm{SO}_{2}$ from the flue gas. FGD wastewaters generally contain significant levels of chloride, total dissolved solids (TDS), total suspended solids (TSS), and nutrients, as well as bioaccumulative pollutants such as arsenic, mercury and selenium. Treatment processes for FGD wastewater include settling

\footnotetext{
" Some plants use dry FGD systems, in which a sorbent slurry is sprayed into a reactor vessel so that droplets dry as they contact the hot flue gas. Although dry FGD scrubbers use water in their operation, the water in most systems evaporates, and dry FGD systems generally do not discharge wastewater.
} 
ponds, chemical precipitation systems, biological treatment systems, constructed wetlands, vapor-compression evaporation systems, and other technologies. ${ }^{365}$

Another source of water pollution is the handling of ash, ${ }^{\text {ww }}$ or the noncombustible residue resulting from coal and oil combustion in steam electric boilers. Fly ash refers to the very fine particles that are light enough to be captured in the flue gas and carried out of the furnace. Fly ash is typically removed from flue gas in electrostatic precipitators or other filters and collected in hoppers beneath the equipment. The heavier ash that settles in the furnace or is dislodged from the furnace walls collects at the bottom of the boiler and is known as bottom ash. Some plants use water to transport the fly ash and bottom ash to a surface impoundment, and the water used for ash transport is usually discharged to surface water as overflow from the systems, after the ash has settled to the bottom. ${ }^{366}$ Untreated ash transport waters contain significant concentrations of TSS and metals, including arsenic, calcium, and titanium.

Flue gas mercury control (FGMC) systems remove mercury from flue gas to reduce air emissions. Depending on the system, FGMC waste will either be collected and handled with existing waste streams (from FGD systems or fly ash systems) or will generate a separate waste stream that can be handled independently from a fly ash system (e.g., as shown in Figure 4.1). FGMC waste that is handled jointly with fly ash can increase the concentration of mercury in the fly ash that is deposited in ash ponds.

Metal cleaning waste, which is defined as "any wastewater resulting from cleaning [with or without chemical cleaning compounds] any metal process equipment, including, but not limited to, boiler tube cleaning, boiler fireside cleaning, and air preheater cleaning" is another wastestream from steam electric power plants (See 40 CFR 423.11). The major constituents of boiler cleaning wastes are the metals of which the boiler is constructed, typically iron, copper, nickel and zinc. Additionally, metal cleaning wastes can include sulfur compounds (in plants burning fossil fuels with high sulfur content) and chemicals that are used to remove scale and corrosion products. Metal cleaning wastes are generated infrequently at many plants, sometimes taking place once every 10 years, and are often sent to an ash pond or other surface impoundment. Some plants have separate wastewater handling processes for metal cleaning wastes because the wastes are generated so infrequently and often have high pollutant concentrations. ${ }^{367}$

Coal pile runoff-runoff from rainwater and melting snow coming into contact with coal pilescan also affect water quality. Power plants generally store between 25 and 40 days' worth of coal in an outdoor coal pile. The quantity of runoff depends upon the amount of precipitation,

\footnotetext{
${ }^{\text {ww }}$ Note that the forthcoming report Solid Waste from the Operation and Decommissioning of Power Plants addresses solid waste and decommissioning waste streams for the electric power industry, including fly and bottom ash. This report only addresses waste handling as it relates to water quality impacts.
} 
the capacity of the plant, the physical location and layout of the pile, and the extent to which water permeates the ground underneath the pile. Flow rates reported for an EPA data request range from 2,070,000 - 364,000,000 gallons per year per plant. ${ }^{368}$ Coal pile runoff is typically acidic due to the oxidation of iron sulfide and ferric hydroxide and may also contain high concentrations of copper, iron, aluminum, nickel, and other contaminants. Plants typically direct coal pile runoff wastewaters to a holding pond along with stormwater runoff from other areas near the coal pile.

Integrated gasification combined cycle (IGCC) plants generate a unique wastewater stream from the gasification process. IGCC plants use a carbon-based feedstock such as coal or petroleum coke and subject it to high temperature and pressure to produce a synthetic gas (syngas), which is used as the fuel for a combined cycle generating unit. After the syngas is produced, it undergoes cleaning prior to combustion to remove particulates, sulfur, chlorides, mercury, and other contaminants. The wastewater generated by these cleaning processes is generally treated prior to reuse or discharge.

Other potential wastestreams from power plants can include wastestreams from alternativefueled plants, nitrogen oxide $\left(\mathrm{NO}_{\mathrm{x}}\right)$ scrubbers, carbon capture technologies, and low volume wastestreams. ${ }^{369}$

\section{Cooling water effluent:}

Additionally, steam electric generating units require a constant flow of cooling water (not shown in Figure 4.1; find more detail in the Energy Water baseline report) to maintain steam condensation and a low pressure in the condenser. Steam electric plants typically use either once-through cooling water systems or recirculating cooling water systems. In once-through cooling water systems, the cooling water is withdrawn from a body of water, flows through a condenser, and is discharged back to the body of water.

In recirculating systems, after water passes through the condenser, the heated water is sent to a cooling tower to lower its temperature. Some of the heated water evaporates, in the process cooling the remaining water and while also leaving behind dissolved minerals that build up within the cooling tower. Fresh water is periodically added to the cooling water system to make up for evaporative losses. Additionally, water must be periodically discharged from the cooling tower in order to prevent mineral buildup-a process referred to as "cooling tower blowdown."

Once-through cooling water and cooling tower blowdown may contain the following pollutants as a result of chlorination and corrosion of the piping, condenser and cooling tower materials: chlorine, iron, copper, nickel, aluminum, boron, chlorinated organic compounds, suspended solids, brominated compounds, and nonoxidizing biocides. While pollutants are often at low 
concentrations, the overall pollutant mass discharge may be significant due to large flow rates. $^{370}$

\section{Leachate from Surface Impoundments and Landfills:}

Many plants treat their wastewater streams through surface impoundments (such as the ash pond in Figure 4.1). Surface impoundments act as a physical treatment process to remove particulate material from wastewater through settling. The wastewater in a surface impoundment can include one specific waste stream (e.g., fly ash transport water) or a combination of wastewaters. Additionally, plants may transfer wastewater streams from other operations into their on-site impoundments (e.g., cooling tower blowdown or metal cleaning wastes). ${ }^{371}$ Solid wastes, including fly ash, bottom ash, boiler slag, and FGD solids - collectively referred to as coal combustion residuals (CCR) - are generally disposed in on-site landfills or transferred off-site for beneficial use ${ }^{\mathrm{xx}}$ or disposal. ${ }^{372}$

Surface impoundments and on-site landfills are both sources of leachate-liquid that drains or leaches from a landfill or an impoundment, and originates either from precipitation that percolates through the waste deposited in the landfill or liquids contained in the original waste. Leachate contains heavy metals and other contaminants as a result of contact with coal combustion residuals. According to an EPA industry survey, most landfills have a system to collect the leachate and transport it to an impoundment (e.g., a collection pond). ${ }^{373}$ Some plants discharge the effluent from these impoundments directly to receiving waters, while other plants send the effluent to another impoundment or other treatment system prior to discharge. Unlined impoundments and landfills usually do not collect leachate, which would allow the leachate to potentially migrate into groundwater, drinking water wells and surface waters. Leachate contains high concentrations of metals, such as boron, calcium, chloride, and sodium, similar to FGD wastewaters and ash transport waters.

\section{Average Discharge Rates:}

Recent EPA analysis characterized average discharge rates and pollutant loadings for a subset of wastestreams, including FGD wastewater, fly and bottom ash transport water, combustion residual leachate. Not all plants have these wastewater streams, and discharge rates and pollutant loadings can vary significantly across the steam electric source category.

\footnotetext{
${ }^{x x}$ Coal plants that use dry ash handling commonly sell their ash wastes for beneficial use in cement and dry wall products.
} 
Table 4.1. Wastewater Discharges for the Steam Electric Power Generating Industry in $2009^{374}$

\begin{tabular}{lcc}
\hline & $\begin{array}{c}\text { Number of plants } \\
\text { discharging }\end{array}$ & $\begin{array}{c}\text { Average discharged } \\
\text { wastewater flow (gpd/plant) }\end{array}$ \\
\hline FGD Wastewater & 100 & 451,000 \\
Fly Ash Transport & 113 & $3,480,000$ \\
Bottom Ash Transport & 283 & $2,050,000$ \\
Combustion Residual Leachate & $100-110$ & $80,000-90,000$ \\
\hline
\end{tabular}

Average wastewater discharges range from $80,000-90,000$ gallons per day for combustion residual leachate to 2-3 million gallons per day for ash handling wastewater.

\subsection{WATER QUALITY IMPACTS FROM POWER GENERATION}

The wastestreams from steam electric power plants contain various categories of pollutants including conventional (such as TSS, biochemical oxygen demand (BOD), and oil and grease), priority (such as mercury (Hg), arsenic (As), and selenium (Se)), and non-conventional pollutants (such as phosphorus (TP), chemical oxygen demand (COD) and TDS). ${ }^{375}$ Impacts from these pollutants fall into several broad categories, including increased health risks, surface and ground water quality, and other environmental impacts. ${ }^{376}$

Of particular concern are toxic bioaccumulative pollutants such as mercury, lead, and other heavy metals because they do not volatilize, do not biodegrade, can be toxic to plants, invertebrates and fish, adsorb to sediments, and bioconcentrate in fish tissues. ${ }^{377}$ Recreational and subsistence fishers (and their household members) are particularly vulnerable to contaminated fish tissue. In a recent study on the impacts of pollutants from steam electric power plants, EPA identified the following impacts to human health from exposure to contaminated fish tissue: ${ }^{378}$

1. Lower IQ in children ages 0 to 7 from exposure to lead;

2. Increased incidence of cardiovascular disease in adults from exposure to lead;

3. Lower IQ in infants from in-utero exposure to mercury;

4. Increased incidence of skin cancer and cardiovascular disease from exposure to arsenic; and

5. Additional risks of cancer and non-cancer toxic effects.

The EPA study also identified other documented health impacts in subsistence and recreational fishers whose effects are difficult to quantify, including: low birth weight and neonatal mortality from in-utero exposure to lead; additional effects to adults from exposure to lead (e.g., nervous system disorders, anemia and blood disorders); 379 effects to adults from exposure to mercury, including vision defects, hand-eye coordination, hearing loss, tremors, cerebellar changes, and 
others (CDC, 2009); and other cancer and non-cancer effects from exposure to other steam electric pollutants.).

Wastewater discharges also impact groundwater and drinking water quality. Exceedances of maximum contaminant levels (MCLs) in drinking water downstream from power plants indicate an ongoing human health concern. In the environmental assessment for the steam electric effluent limitation guidelines, EPA identified more than 30 documented cases where groundwater contamination from surface impoundments extended beyond the plant boundaries. $^{380}$

Surface water discharges also have a wide variety of environmental effects, including fish kills, reduction in the survival and growth of aquatic organisms, behavioral and physiological effects in wildlife, and degradation of aquatic habitat. Threatened and endangered species are particularly vulnerable to factors that cause even minor changes to reproductive rates and levels of mortality. ${ }^{381}$

Table 4.2 lists some of the common metals found in steam electric power plant wastewater and potential health and environmental impacts associated with each pollutant. This list is not comprehensive of all metals in power plant wastestreams, nor does it include all potential environmental impacts for each metal. 
Table 4.2. Key Metals and Other Pollutants Found in Power Plant Wastewater. ${ }^{382}$

\begin{tabular}{|c|c|}
\hline Pollutant & Examples of Potential Health and Environmental Concerns \\
\hline Aluminum & $\begin{array}{l}\text { Aluminum contamination is associated with damage to amphibian eggs and larvae. Human exposure to high } \\
\text { concentrations has been linked to Alzheimer's disease. }\end{array}$ \\
\hline Arsenic & $\begin{array}{l}\text { Arsenic is associated with an increased risk of the liver and bladder cancer in humans. Arsenic is also a potent } \\
\text { endocrine disruptor at low, environmentally relevant levels. Non-cancer impacts to humans can include } \\
\text { dermal, cardiovascular, and respiratory effects. Negative impacts can occur both after high-dose exposure } \\
\text { and repeated lower-dose exposures. Chronic exposure via drinking water has been associated with excess } \\
\text { incidence of miscarriages, stillbirths, preterm births, and low-birth weights. }\end{array}$ \\
\hline Boron & $\begin{array}{l}\text { Boron can be toxic to vegetation and to wildlife at certain water concentrations and dietary levels. Human } \\
\text { exposure to high concentrations can cause nausea, vomiting, and diarrhea. }\end{array}$ \\
\hline Cadmium & $\begin{array}{l}\text { Cadmium contamination can lead to developmental impairments in wildlife and skeletal malformations in } \\
\text { fish. Human exposure to high concentrations in drinking water and food can irritate the stomach, leading to } \\
\text { vomiting and diarrhea, and sometimes death. Chronic oral exposure via diet or drinking water to lower } \\
\text { concentrations can lead to kidney damage and weakened bones. }\end{array}$ \\
\hline Chlorides & $\begin{array}{l}\text { Excess chloride levels can be harmful to animals and plants and can disrupt ecosystem structure. High } \\
\text { concentrations in surface waters can also impair their use as source waters for potable water supplies. }\end{array}$ \\
\hline Chromium & Human exposure to high concentrations can cause gastrointestinal bleeding and lung problems. \\
\hline Copper & Human exposure to high concentrations can cause nausea, vomiting, diarrhea, and liver and kidney damage. \\
\hline Iron & $\begin{array}{l}\text { Human exposure to high concentrations can cause metabolic changes and damage to the pancreas, liver, } \\
\text { spleen, and heart. }\end{array}$ \\
\hline Lead & $\begin{array}{l}\text { Human exposure to high concentrations in drinking water can cause serious damage to the brain, kidneys, } \\
\text { nervous system, and red blood cells. }\end{array}$ \\
\hline Manganese & $\begin{array}{l}\text { Manganese primarily accumulates in organisms lower in the food chain such as phytoplankton, algae, } \\
\text { mollusks, and some fish. The most common impacts due to human exposure to high concentrations involve } \\
\text { the nervous system. }\end{array}$ \\
\hline Mercury & $\begin{array}{l}\text { Once in the environment, mercury can convert into methylmercury, increasing the potential for } \\
\text { bioaccumulation. Methylmercury contamination can reduce growth and reproductive success in fish and } \\
\text { invertebrates. Human exposure at levels above the maximum contaminant level (MCL) for relatively short } \\
\text { periods can result in kidney and brain damage. Fetuses, infants, and children are particularly susceptible to } \\
\text { impaired neurological development from methylmercury exposure. }\end{array}$ \\
\hline Nickel & $\begin{array}{l}\text { Nickel toxicity in fish and aquatic invertebrates varies among species and can damage the lungs, immune } \\
\text { system, liver, and kidneys. Human exposure to high concentrations can cause gastrointestinal and kidney } \\
\text { damage. }\end{array}$ \\
\hline Selenium & $\begin{array}{l}\text { Selenium readily bioaccumulates. Elevated concentrations have caused fish kills and numerous sublethal } \\
\text { effects (e.g., organ damage, decreased growth rates, reproductive failure) to aquatic and terrestrial } \\
\text { organisms. In humans, short-term exposure at levels above the (MCL) can cause hair and fingernail changes, } \\
\text { damage to the peripheral nervous system, and fatigue and irritability. Long-term exposure can damage the } \\
\text { kidney, liver, and nervous and circulatory systems. }\end{array}$ \\
\hline Thallium & $\begin{array}{l}\text { In humans, short-term exposure to thallium can lead to neurological symptoms, alopecia, gastrointestinal } \\
\text { effects, and reproductive and developmental damage. Long-term exposures at levels above the (MCL) } \\
\text { change blood chemistry and damage liver, kidney, intestinal and testicular tissues and cause hair loss. }\end{array}$ \\
\hline Vanadium & $\begin{array}{l}\text { Vanadium contamination can increase blood pressure and cause neurological effects in animals. In humans, } \\
\text { vanadium exposure has been linked to the development of some neurological disorders and cardiovascular } \\
\text { diseases. }\end{array}$ \\
\hline Zinc & anem \\
\hline
\end{tabular}

Key pollutants found in steam electric wastewater streams include toxic bioaccumulative metals, as well as chlorides, nutrients, and other dissolved and suspended solids.

In addition to the pollutants listed in Table 4.2, wastewater from steam electric power plants also contains nutrients and other dissolved and suspended solids which, while not generally considered toxic, can have negative impacts on plants and animals. Nutrients (e.g., phosphorus 
and nitrogen) are essential components for wildlife to grow and develop; however, increased nutrient concentrations can cause algal blooms and rapid growth of plants and cyanobacteria on the waterbody surface, which can obstruct sunlight penetration, increase turbidity, and decrease dissolved oxygen levels. ${ }^{383}$

The toxicity of pollutants varies significantly by type. To allow for comparison across pollutants, the amount of discharged pollutants (i.e., loadings) is typically reported in both pounds (Ib) and toxic weighted pound equivalent units (Ib-eq). EPA calculates toxic weighted pound equivalent (TWPE) by multiplying the amount of a pollutant in pounds per year (lb/yr) by a pollutantspecific weighting factor, call the toxic weighting factor (TWF), to derive a "toxic equivalent" loading, or TWPE. TWFs account for differences in toxicity across pollutants and allow amounts of different pollutants to be compared on the basis of their toxic potential.

EPA estimates that discharges from steam electric power plants account for over one-third of all discharges in TWPE units. In a baseline analysis of current pollution levels, EPA estimates the pollution from steam electric power plants at 2,210,000,000 pounds annually, equivalent to $2,680,000$ pounds of TWPE per year. Table 4.3 displays annual baseline pollutant discharges by pollutant in pounds and TWPE. 
Table 4.3. Annual Baseline Pollutant Discharges from Steam Electric Power Plants. ${ }^{384}$ yy

\begin{tabular}{lccc}
\hline Pollutant & $\begin{array}{c}\text { Toxic Weighting } \\
\text { Factor }\end{array}$ & $\begin{array}{c}\text { Annual Discharge, } \\
\text { pounds (lbs) }\end{array}$ & $\begin{array}{c}\text { Annual TWPE, } \\
\text { pound-equivalent } \\
\text { (Ib-eq) }\end{array}$ \\
\hline Manganese & 0.103 & $7,530,000$ & 773,000 \\
Cadmium & 22.8 & 13,300 & 303,000 \\
Boron & 0.00834 & $31,300,000$ & 261,000 \\
Thallium & 2.85 & 63,700 & 182,000 \\
Mercury & 110.0 & 1,490 & 164,000 \\
Selenium & 1.12 & 140,000 & 157,000 \\
Arsenic & 3.47 & 29,600 & 103,000 \\
Aluminum & 0.0647 & $1,410,000$ & 91,500 \\
Lead & 2.24 & 19,700 & 44,100 \\
Copper & 0.623 & 31,200 & 19,500 \\
Vanadium & 0.280 & 66,000 & 18,500 \\
Iron & 0.00560 & $2,740,000$ & 15,400 \\
Nickel & 0.109 & 120,000 & 13,100 \\
Zinc & 0.0469 & 174,000 & 8,160 \\
Chromium VI & 0.517 & 156 & 80.5 \\
Chlorides & $2.435 \times 10^{-5}$ & $930,000,000$ & 22,600 \\
Total Pollutants & & $2,210,000,000$ & $2,680,000$ \\
\hline
\end{tabular}

Baseline discharges from steam electric power plants are estimated to be 2,680,000 pounds of TWPE per year, nearly three times the amount estimated for the pulp, paper, and paperboard industry, the petroleum refining industry, and nonferrous metals manufacturing industry (second, third, and fourth highest ranking source categories).

The total TWPE discharges from the steam electric power generating industry are higher than the TWPE discharges from other industries. As part of the Preliminary 2010 Effluent Guidelines Program Plan, EPA ranked steam electric power plants as the largest source of TWPE discharges out of 56 point source categories. EPA estimated that total baseline TWPE from steam electric power plant wastewater is almost three times the amount estimated for the pulp, paper, and paperboard industry $(1,030,000 \mathrm{lb}-\mathrm{eq} / \mathrm{yr})$, petroleum refining industry $(1,030,000 \mathrm{lb}-\mathrm{eq} / \mathrm{yr})$, and nonferrous metals manufacturing $(994,000 \mathrm{lb}-\mathrm{eq} / \mathrm{yr})$, which came in second, third, and fourth among point source categories. ${ }^{385}$

\footnotetext{
yy The list of pollutants included in this table is only a subset of pollutants found in steam electric power plant wastestreams.
} 
Annual TWPE discharges can also be tracked using EPA's Discharge Monitoring Report (DMR) pollutant loading tool. The tool uses data from EPA's Integrated Compliance Information System for the National Pollutant Discharge Elimination System (ICIS-NPDES) and also includes wastewater pollutant discharge data from EPA's Toxic Release Inventory (TRI). Discharges from steam electric generating plants can vary substantially from year to year. Factors affecting total reported discharges include changes in the generation mix, the efficiency of electricity generation, and the quality of fuels used. Figure 4.2 shows annual reported discharges in toxicweighted pound equivalent (TWPE) units for steam electric generating plants from 2007-2015. Note that the totals reported here represent total pollutants, of which the list in Table 4.2 is only a subset.

Figure 4.2. Annual Reported TWPE Discharges from Steam Electric Generating Plants, 2007$2015 . .^{386}$

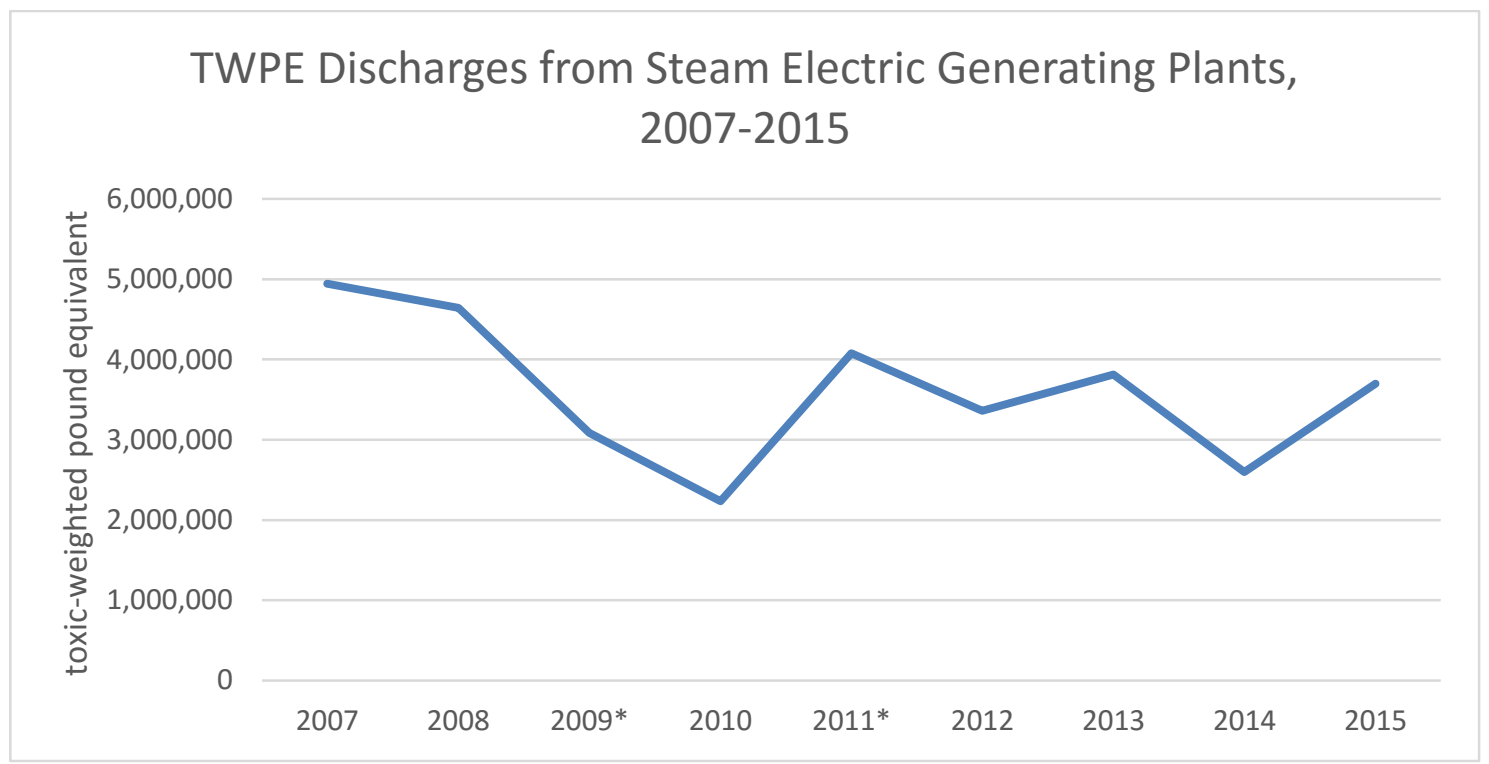

In 2015, 524 facilities reported discharging 6.9 billion pounds of total dissolved solids, or 3.7 million pounds in toxic-weighted pound equivalent units.

Note: Total power sector discharges are determined by aggregating facility-level totals reported in the Discharge Monitoring Report tool. Discharges from two power plants in 2009 and one power plant in 2011 have been tagged as outliers and have been removed from sector totals until discharges can be verified.

The Discharge Monitoring Report tool also provides facility-level information about pollutant discharges from steam electric generating plants. In 2015, 524 facilities reported discharging $6,862,875,980$ pounds of total dissolved solids, or 3,679,356 pounds in toxic-weighted pound equivalent units. 
Figure 4.3. Map of Discharging Facilities in the Steam Electric Power Plants Point Source Category, 2015. 387

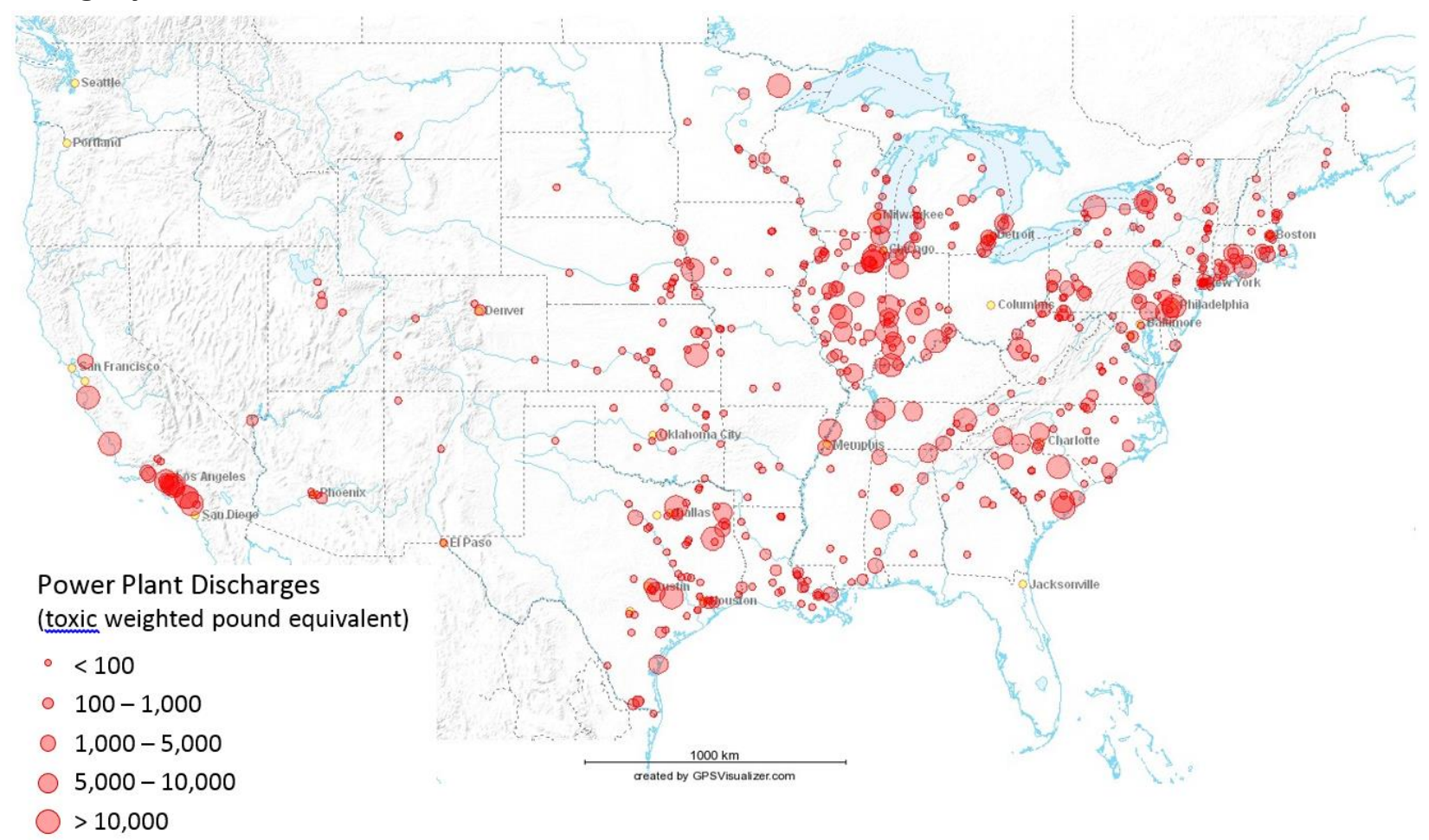

The facilities with the highest levels of TWPE discharges are located in the South, Midwest, Northeast and California. New York (81) and Texas (80) have the largest numbers of facilities reporting discharges in the steam electric power plants category.

\subsection{POLICIES REGULATING WATER QUALITY IMPACTS FROM STEAM ELECTRIC POWER GENERATION}

Major policies regulating water quality impacts from steam electric power generation include the effluent limitations guidelines (ELGs) promulgated under the Clean Water Act, and the coal combustion residuals (CCR) disposal requirements promulgated under the Resource Conservation and Recovery Act.

Most recently, EPA updated the effluent limitations guidelines in September 2015. The rule establishes new requirements for wastewater streams from the following processes and byproducts associated with steam electric power generation: flue gas desulfurization, fly ash, bottom ash, flue gas mercury control, and gasification of fuels such as coal and petroleum coke. The rule establishes new, more stringent effluent limits for arsenic, mercury, selenium, and nitrogen for wastewater discharged from wet scrubber systems (FGD wastestream). In addition, the rule sets zero discharge pollutant limits for flue gas mercury control (FGMC) wastewater, and stringent limits on arsenic, mercury, selenium, and total dissolved solids. 
The new effluent guidelines are projected to reduce the amount of toxic metals, nutrients, and other pollutants that steam electric power plants are allowed to discharge by 1.4 billion pounds annually, and will reduce annual water withdrawal by 57 billion gallons.

Projected pollutant removals are greatest for the most toxic pollutants (i.e., the pollutants listed in Table 4.2). Under the final rule, EPA estimates that pollutant loadings from existing sources will decrease by over 95 percent for copper, lead, mercury, nickel, selenium, thallium, and zinc and over 90 percent for arsenic and cadmium. Total TWPE discharges for the pollutants in Table 4.2 are projected to decline by 63 percent, from 2,680,000 TWPE/year in the baseline to 1,000,000 TWPE/yr under the new effluent guidelines (Table 4.4).

Table 4.4. Estimated Pollutant Loadings and Removals under the Steam Electric Effluent Limitation Guidelines (ELGs) ${ }^{388}$

\begin{tabular}{lcc}
\hline & \multicolumn{2}{c}{ Total Industry Loading } \\
& Pounds/Year & TWPE/Year \\
\hline Baseline & $2,210,000,000$ & $2,680,000$ \\
Pollutant removals under the rule* & $513,000,000$ & $1,680,000$ \\
Steam Electric ELGs & $1,700,000,000$ & $1,000,000$ \\
\hline
\end{tabular}

As the updated steam electric effluent limitations and guidelines (ELGs) go into effect, annual TWPE discharges are projected to decline by 63 percent, from 2,680,000 TWPE/year in the baseline to $1,000,000$ TWPE/year under the new regulations. Each steam electric plant must reach compliance between 2018 and 2023 depending on when it needs a new discharge permit.

Note: Pollutants accounted for in these estimates include only the pollutants listed in Table 4.2. The rule is also projected to reduce nitrogen loadings by $16.8 \mathrm{million} \mathrm{lbs} / \mathrm{yr}$ (99 percent), phosphorus loadings by $174,000 \mathrm{lbs} / \mathrm{yr}$ (81 percent), and total dissolved solids (TDS) loadings by 1.32 billion lbs/yr (31 percent).

ELGs also exist from earlier rulemakings for once through cooling water, cooling tower blowdown, chemical metal cleaning wastes, low volume wastes, and coal pile runoff. ${ }^{389}$ Effluent limitations guidelines and standards are enforced through the National Pollutant Discharge Elimination System (NPDES) and are incorporated into NPDES permits issued by States and EPA regional offices. ${ }^{390}$ Under the updated steam electric ELGs, each plant must comply between 2018 and 2023 depending on when it needs a new NPDES permit.

Another major policy is the CCR rule, which EPA promulgated in December 2014, to specifically address risks to groundwater quality from leaking impoundments. Described in more detail in the Solid Waste baseline report, ${ }^{391}$ this rule establishes technical requirements for CCR surface impoundments, including composite liners, groundwater monitoring, corrective action, and closure/post closure care, among others. For example, the rule requires any existing unlined CCR surface impoundment that is contaminating groundwater above a regulated constituent's groundwater protection standard to stop receiving CCR and either retrofit or close, and that 
corrective action be taken to address contamination from leaking clay- or composite-lined impoundments. ${ }^{392}$ 


\section{ENVIRONMENTAL JUSTICE}

Environmental justice (EJ) is the fair treatment and meaningful involvement of all people regardless of race, color, national origin, or income with respect to the development, implementation, and enforcement of environmental laws, regulations, and policies. ${ }^{393}$

Fair treatment means that no group of people should bear a disproportionate burden of environmental harms and risks, including those resulting from the negative environmental consequences of industrial, governmental and commercial operations or programs and policies. Meaningful involvement means that: (1) potentially affected populations have an appropriate opportunity to participate in decisions about a proposed activity that will affect their environment and/or health; (2) the public's contribution can influence the regulatory Agency's decision; (3) the concerns of all participants involved will be considered in the decision-making process; and (4) the rule-writers and decision-makers seek out and facilitate the involvement of those potentially affected. ${ }^{394}$

Environmental justice is achieved when everyone enjoys the same degree of protection from environmental and health hazards and equal access to the decision-making process to have a healthy environment in which to live, learn, and work. 395396

In general, the environmental impacts from electricity generation can vary substantially across population subgroups. A population's vulnerability to environmental impacts is a function of its exposure to environmental risks, its inherent sensitivity to those risks, and its capacity to respond to environmental impacts. ${ }^{397}$

Sensitivity is the degree to which people or communities are affected by environmental quality and is determined, at least in part, by biologically based traits such as age. ${ }^{398}$ For example, older adults and children are physiologically more sensitive to health risks. In addition, underlying health disparities can contribute to biological sensitivity and are more prevalent in low-income populations and some communities of color. For example, African American populations have higher rates of chronic conditions such as asthma, decreased lung function, and cardiovascular issues, which are known to increase sensitivity to health effects of air pollution from power plants. $^{399}$

Adaptive capacity is the ability to adjust to potential hazards and can vary widely across communities and individuals. People with limited economic resources living in areas with deteriorating infrastructure are more likely to experience disproportionate impacts and are less able to recover following extreme events, increasing their vulnerability to climate-related health effects. ${ }^{400}$ 
Greater exposure or greater sensitivity to environmental impacts, or reduced adaptive capacity within a population may indicate potential environmental justice concerns. EJ concerns related to the electric power industry include the impacts to air quality and water quality, the impacts of climate change, and equal access to affordable energy and ratepayer impacts.

\subsection{EJ CONSIDERATIONS/IMPACTS RELATED TO POWER GENERATION}

\subsubsection{Air Quality EJ Concerns}

Power plants are the largest U.S. source of several harmful pollutants. For example, they are responsible for $64 \%$ of economy-wide $\mathrm{SO}_{2}$ emissions, ${ }^{401}$ about 50 percent of mercury emissions and 77 percent of acid gas emissions including hydrochloric acid $(\mathrm{HCl})$ and hydrofluoric acid (HF). ${ }^{402}$ At sufficient levels of exposure, these pollutants can cause a range of health effects including cancer; irritation of the lungs, skin, and mucous membranes; effects on the central nervous system such as memory and IQ loss and learning disabilities; damage to the kidneys; and other acute health disorders. Additionally, power plants are significant sources of criteria air pollutants, which cause premature mortality for adults, chronic and acute bronchitis, childhood asthma attacks, and exacerbate other respiratory and cardiovascular diseases. ${ }^{403}$

The impacts of pollution on populations of concern vary substantially across communities and depend on several factors, including exposure, inherent sensitivity, and adaptive capacity to respond to air pollution. For some pollutants, proximity-to-the-source is commonly used as a surrogate for exposure to hazardous air pollutant emissions from these sources. For example, some electricity generating units emit enough nickel or chromium to cause elevated lifetime cancer risks greater than 1 in a million in nearby communities. In addition, EPA analysis indicates that there are localized areas with elevated levels of mercury deposition around most U.S. power plants. ${ }^{404}$

Table 5.1 shows the demographics of populations living within 3 miles of coal- and oil-fired power plants. The minority share of the population living within a three-mile buffer is higher than the national average by 12 percentage points, or $37 \%$. In these same areas, the percent of the population below the poverty line is also higher than the national average by 4 percentage points, or $17 \% .{ }^{405}$ 
Table 5.1. Comparative Summary of the demographics within 5 kilometers ( 3 miles) of the affected sources (population in millions)..$^{406}$

\begin{tabular}{|c|c|c|c|c|c|c|c|c|c|c|c|}
\hline & $\begin{array}{l}\frac{5}{0} \\
\frac{0}{0} \\
\frac{\pi}{0} \\
\frac{0}{0}\end{array}$ & 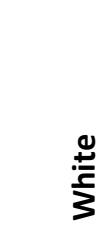 & 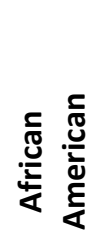 & 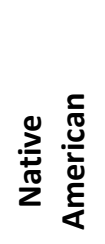 & 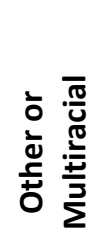 & 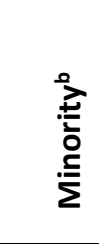 & 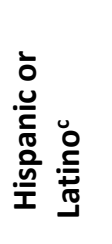 & 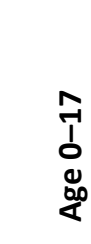 & 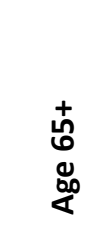 & 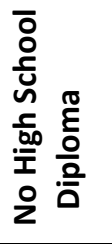 & 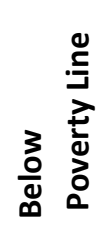 \\
\hline Near source total (3 mi) & 13.9 & 8.78 & 2.51 & 0.10 & 2.52 & 5.13 & 2.86 & 3.37 & 1.65 & 2.20 & 2.43 \\
\hline$\%$ of near source total & & $63 \%$ & $18 \%$ & $1 \%$ & $18 \%$ & $37 \%$ & $21 \%$ & $24 \%$ & $12 \%$ & $16 \%$ & $17 \%$ \\
\hline National total & 285 & 215 & 35.0 & 2.49 & 33.3 & 70.8 & 39.1 & 77.4 & 35.4 & 36.7 & 37.1 \\
\hline$\%$ of national total & & $75 \%$ & $12 \%$ & $1 \%$ & $12 \%$ & $25 \%$ & $14 \%$ & $27 \%$ & $12 \%$ & $13 \%$ & $13 \%$ \\
\hline
\end{tabular}

The minority share of the population living within a three-mile buffer of power plants is higher than the national average by 12 percentage points, or $37 \%$. In these same areas, the percent of the population below the poverty line is also higher than the national average by 4 percentage points, or $17 \%$.

Notes: aRacial and ethnic categories overlap and cannot be summed. ${ }^{\text {b}}$ The "Minority" population is the overall population (in the first column) minus white population (in the second column). "The Census Bureau defines "Hispanic or Latino" as an ethnicity rather than a racial category, Hispanics or Latinos may belong to any race.

The sensitivity, or degree to which people or communities are affected by air pollution depends on a number of factors including age, access to healthcare, baseline health status, and other characteristics. In the United States, African Americans, women, children, and the elderly experience the greatest sensitivity to the impacts of air pollution. ${ }^{407}$ For example, low-income urban populations have relatively higher rates of cardiovascular and respiratory diseases and are more sensitive to degraded air quality. In 2000, the prevalence of asthma was 122 per 1,000 African Americans and 104 per 1,000 Caucasian Americans. At the time, asthma mortality was approximately three times higher among African Americans as compared to Caucasian Americans. $^{408}$

Several factors make children more sensitive to air quality impacts, including lung development that continues through adolescence, the size of the child's airways, their level of physical activity, and body weight. Ground-level ozone and particulate matter are associated with increases in asthma episodes and other adverse respiratory effects in children. Particulate matter such as dust and emissions from coal-fired power plants is also associated with decreases in lung maturation in children. Infants and toddlers are particularly sensitive to air pollutants, and ozone exposure in young children is associated with increased asthma risk and other respiratory illnesses. ${ }^{409}$ 
Minority adults and children also bear a disproportionate burden associated with asthma as measured by emergency department visits, lost work and school days, and overall poorer health status. Twice as many African American children have asthma-related emergency department visits and hospitalizations compared with Caucasian American children. Fewer African American and Hispanic children report using preventative medication like inhaled corticosteroids (ICS) as compared to Caucasian American children. African American and Hispanic children also have more poorly controlled asthma symptoms, leading to increased emergency department visits and greater use of rescue medications rather than routine daily use of ICS. ${ }^{410}$

People with preexisting medical conditions-including hypertension, diabetes, and chronic obstructive pulmonary disorder-are at greater risk for outdoor air pollution-related health effects than the general population.

In addition, some EJ populations in the United States are more highly exposed to mercury than the general population. Populations of concern include lower income people who regularly supplement their diets with locally caught fish as well as certain ethnic groups who consume more fish than average for cultural or subsistence reasons. These include members of certain native tribal groups, and certain populations of Hispanic or Asian background. See the section on Water Quality for more information.

\subsubsection{Water Quality}

Energy production and water are highly interdependent. Energy production is the second largest user of water in the United States, and virtually every type and phase of production requires water, from the mining of fossil fuels to the cooling water used steam electric power plants. ${ }^{z z}$ This can lead to the depletion of local water sources and competition for use in agriculture and public drinking water. This competition for fresh water may be especially acute among EJ communities. ${ }^{411}$

Additional water quality impacts from electricity generation include impacts from both the direct emissions of wastewater effluents from electric generating units, and the wet deposition of air emissions (such as mercury) from flue gas.

The impacts of wastewater pollutants vary by the susceptibility of population subgroups in affected areas and can be affected by several factors, including population characteristics, proximity to affected waters, exposure pathways, cumulative risk exposure, and sensitivity to environmental risk. For example, subsistence fishers rely on self-caught fish for a larger share of their food intake than do recreational fisherman, and may be more susceptible to the health

\footnotetext{
${ }^{z z}$ For more, see the Water-Energy Nexus.
} 
impacts from water pollutants than the general population. ${ }^{412}$ Tribal populations are especially vulnerable to the effects of bioaccumulative metals such as mercury through their consumption of fresh water fish and shellfish. ${ }^{413}$

Recent EPA analysis examines the EJ implications of steam electric power plant discharges, including the human health impacts from consuming self-caught fish on minority and/or lowincome populations located within specified distances of reaches affected by steam electric power plant discharges. The receiving reaches are those to which plants discharge directly. EJ concerns may exist in areas where the percent of the population below the poverty threshold is higher than the state or national average or the percent of the population that is minority is above the state or national average.

Table 5.2. Socio-economic Characteristics of Communities Living in Proximity to Receiving Reaches of Surface Water Discharge from Steam Electric Power Plants. ${ }^{414}$

\begin{tabular}{lccc}
\hline $\begin{array}{c}\text { Distance from } \\
\text { receiving reach }\end{array}$ & $\begin{array}{c}\text { Total population } \\
\text { (millions) }\end{array}$ & Percent minority & $\begin{array}{c}\text { Percent below } \\
\text { poverty level }\end{array}$ \\
\hline 1 mile & 0.2 & $20.7 \%$ & $16.4 \%$ \\
3 miles & 1.1 & $23.4 \%$ & $15.3 \%$ \\
15 miles & 14.0 & $29.8 \%$ & $13.6 \%$ \\
30 miles & 37.4 & $31.5 \%$ & $13.1 \%$ \\
50 miles & 57.3 & $29.9 \%$ & $13.1 \%$ \\
United States & $\mathbf{3 0 6 . 3}$ & $\mathbf{3 6 . 0 \%}$ & $\mathbf{1 3 . 9 \%}$ \\
\hline
\end{tabular}

A greater fraction of the communities living in close proximity to steam electric power plants is poor, when compared to the national average. Approximately 16.4 percent of households in communities within 1 mile of steam electric power plants have income below the poverty level as compared to a national average of 13.9 percent.

As shown in Table 5.2, approximately 200,000 people live within 1 mile of the receiving reaches of surface water discharge from steam electric power plants, over 1.1 million live within 3 miles, and nearly 37.4 million people live within 30 miles. The statistics also show that a higher fraction of the communities living in close proximity to steam electric power plants is poor, when compared to the national average. Approximately 16.4 percent of households in communities within 1 mile of receiving reaches of steam electric power plant discharges have incomes below the poverty level as compared to a national average of 13.9 percent. ${ }^{415}$

A smaller fraction of the population within 1 mile of the receiving reaches belongs to minority racial or ethnic groups (20.7 percent), than the national average (36.0 percent). As one moves further away from the steam electric power plants, the fraction of the community that is below 
the poverty threshold goes down while the percent minority increases, so that the overall composition of the communities approaches that of the U.S. population overall.

EPA also found important regional differences in the distribution of affected populations. Of the 37 states with communities within 1 mile of receiving reaches of steam electric power plant discharges, 11 states have communities with a higher percentage of households below the poverty threshold than the overall state, 17 have a higher percent of the population that is minority, and 10 have a higher proportion of poor and minority households. ${ }^{416}$

The distribution of adverse health effects from steam electric pollutants is a function of the characteristics of the affected population, ${ }^{\text {aaa }}$ including age and sex, ${ }^{\text {bbb }}$ ethnicity-specific exposure factors, ${ }^{c c c}$ and reach water quality. Of the approximately 36.0 million people exposed to steam electric pollutants, 6.5 percent are both poor and minority, 35.3 percent are poor or minority, and 58.1 percent are non-poor white. ${ }^{417}$

In the MATS regulatory impact analysis, EPA provides a qualitative assessment of the EJ impacts of (air) mercury emissions from electric generating units on people who rely on freshwater fish they catch as a regular and frequent part of their diet. These groups are characterized as subsistence level fishing populations or fishers. Based on a review of the literature, EPA analysis focused on six subpopulations at high potential risks for mercury exposure due to high rates of fish consumption: ${ }^{418}$

- low-income African-American recreational/subsistence fishers in the Southeast region;

- low-income white recreational subsistence fishers in the Southeast region;

- low-income female recreational/subsistence fishers;

- Hispanic subsistence fishers;

- Laotian subsistence fishers; and

- Chippewa/Ojibwe Tribe members in the Great Lakes area.

These subpopulations were identified based on published empirical evidence of particularly high self-caught freshwater fish consumption rates among these groups. Average consumption rates of self-caught freshwater fish for these subpopulations ranged from 25.8 grams per day (g/day) to $171 \mathrm{~g} /$ day. Because of their high rates of fish consumption, reductions in mercury

\footnotetext{
aaa "Affected communities" are census block groups within 50 miles of one or more receiving reaches of surface water discharge from steam electric power plants.

bbb Some adverse health effects were analyzed only for individuals in certain age groups. For example, IQ point decrements from exposure to lead are calculated for children 0 to 7 years old and the baseline exposure therefore depends on the number of children within this age group in the affected population within each socioeconomic subgroup. IQ point decrements from exposure to mercury were calculated to for infants born within the analysis period and depends on the number of women of childbearing age (and fertility rates) in the affected population. ${ }^{c c c}$ Ethnicity-specific factors that determine exposure to pollutants in fish tissue include the assumed fish consumption rates and the average fertility rate.
} 
occurring in waterbodies where these populations catch fish will have a larger IQ benefit for these populations relative to the general fish consuming population.

\subsubsection{Climate Vulnerabilities to Environmental Justice Communities}

In addition to other air pollutants, the power sector is also the largest source of U.S. greenhouse gas (GHG) emissions. ${ }^{419}$ GHGs absorb some of the heat radiated from the earth's surface and then re-radiate this heat back toward the surface, essentially acting like a blanket that makes the earth's surface warmer than it would otherwise be. ${ }^{420}$ As the concentration of carbon dioxide and other GHGs increase in the earth's atmosphere, the earth's temperature is increasing above previous levels, and the earth's climate is changing in other ways that can adversely impact human health. ${ }^{421} 422$

The Intergovernmental Panel on Climate Change Fifth Assessment Report discusses a range of observed climate impacts that may affect communities vulnerable to EJ issues. These include shifts in water resource quantity and quality due to precipitation and glacial melt changes, species range shifts, increasing occurrence of negative crop yield impacts, increased heatrelated mortality, various climate-related extremes such as wildfires and extreme weather patterns, and other impacts. The IPCC report finds that "People who are socially, economically, culturally, politically, institutionally, or otherwise marginalized are especially vulnerable to climate change and also to some adaptation and mitigation responses." ${ }^{423}$ In the United States, recent examples of extreme weather and other impacts-including Hurricane Katrina, Hurricane Sandy, the California drought, and others-have exposed many communities to hardship. Such incidents are not limited to EJ communities, but their impacts may be greater or longer lasting because of increased sensitivity to climate impacts and because of reduced adaptive capacity (i.e., the ability to respond to adverse circumstances).

The EPA summarized the public health and welfare impacts from GHG emissions in its 2009 Endangerment Finding under the Clean Air Act section 202(a)(1). ${ }^{424}$ Populations that were found to be particularly vulnerable to climate change risks include the poor, the elderly, the very young, those already in poor health, the disabled, those living alone, and/or indigenous populations dependent on one or a few resources. Poor communities were found to be especially vulnerable to climate change impacts because they tend to have more limited adaptive capacities and are more dependent on climate-sensitive resources such as local water and food supplies. These findings were affirmed more recently in the Regulatory Impact Analysis for the Clean Power Plan, ${ }^{425}$ which found that certain climate change related impactsincluding heat waves, degraded air quality, and extreme weather events-have disproportionate effects on low-income populations and some communities of color. Existing 
health disparities and other inequities in these communities increase their vulnerability to the health effects of climate change.

The U.S. Global Change Research Program recently examined the health impacts of climate change, and also identified vulnerable populations with increased health risks. Among the key findings: ${ }^{426} 427$

- Health impacts associated with climate-related changes in exposure to extreme events include death, injury, or illness; exacerbation of underlying medical conditions; and adverse effects on mental health.

- Climate change will increase exposure risk in some regions of the United States due to projected increases in the frequency and/or intensity of drought, wildfires, and flooding related to extreme precipitation and hurricanes.

Table 5.3 summarizes some of the climate-related extreme weather events that are projected to increase, along with associated health risks and populations of concern. 
Table 5.3. Health Impacts of Climate-Related Extreme Events ${ }^{428}$

\begin{tabular}{|c|c|c|}
\hline Event Type & Example Health Risks and Impacts & $\begin{array}{c}\text { Populations of Concern and EJ } \\
\text { Considerations }\end{array}$ \\
\hline Extreme heat events & $\begin{array}{l}\text { - Premature heat-related deaths in the } \\
\text { summer, projected to increase at a } \\
\text { rate of thousands to tens of } \\
\text { thousands each year. } \\
\text { - Heat-related illnesses, including } \\
\text { cardiovascular, respiratory, and renal } \\
\text { illnesses; hyperthermia, and preterm } \\
\text { births. }\end{array}$ & $\begin{array}{l}\text { Older adults and children have a higher } \\
\text { risk of dying or becoming ill due to } \\
\text { extreme heat. } \\
\text { Some minority and low-income } \\
\text { I communities are more exposed to heat } \\
\text { waves and have limited adaptive capacity } \\
\text { due to lack of adequately insulated } \\
\text { housing or air conditioning. African- } \\
\text { Americans have elevated risks for } \\
\text { mortality from extreme heat exposure, as } \\
\text { compared to Caucasian-Americans. }\end{array}$ \\
\hline $\begin{array}{l}\text { Flooding related to extreme } \\
\text { precipitation, hurricanes, } \\
\text { coastal storms }\end{array}$ & $\begin{array}{l}\text { - } \\
\text { (draumatic injury and death } \\
\text { - } \quad \text { Preterm birth and low birth weight } \\
\text { - } \quad \text { Infrastructure disruptions and post- } \\
\text { event disease spread } \\
\text { - Carbon monoxide poisoning related } \\
\text { to power outages }\end{array}$ & $\begin{array}{l}\text { As observed after Hurricane Katrina and } \\
\text { Sandy, some communities of color and } \\
\text { low-income people experienced increased } \\
\text { illness, injury, or death due to poor-quality } \\
\text { housing, lack of access to transportation, } \\
\text { and lack of access to emergency services } \\
\text { and health care services and medications. }\end{array}$ \\
\hline Droughts & $\begin{array}{ll}\text { - } & \text { Reduced water quality and quantity } \\
\text { - } & \text { Respiratory impacts related to } \\
& \text { reduced air quality }\end{array}$ & $\begin{array}{l}\text { Many indigenous people living on remote } \\
\text { reservations lack indoor plumbing and rely } \\
\text { on unregulated water supplies that are } \\
\text { vulnerable to drought and changes in } \\
\text { water quality. }\end{array}$ \\
\hline Wildfires & $\begin{array}{ll}\text { - } & \text { Smoke inhalation } \\
\text { - } & \text { Burns and other traumatic injury } \\
\text { - } & \text { Asthma exacerbations } \\
\text { - } & \text { Increased ground-level ozone and } \\
& \text { particulate matter, leading to } \\
\text { degraded air quality }\end{array}$ & $\begin{array}{l}\text { Pregnant women, children and the elderly } \\
\text { are particularly sensitive to wildfire smoke } \\
\text { exposure. } \\
\text { Minority adults and children bear a } \\
\text { disproportionate burden associated with } \\
\text { asthma. Children living in poverty are } 1.75 \\
\text { times more likely to be hospitalized for } \\
\text { asthma than their non-poor counterparts. }\end{array}$ \\
\hline
\end{tabular}

\subsection{EJ POLICIES}

The responsibility of Federal agencies to consider environmental justice is set out in Executive Order No. $12898^{429} 430$ and typically implemented in conjunction with NEPA review, as described in the Council on Environmental Quality (CEQ) guidance for environmental justice. ${ }^{431}$

As the primary environmental regulatory agency, EPA has worked to integrate environmental justice in all of its programs, including rulewriting, permitting, enforcement, science and law. ${ }^{432}$ 
Recent EPA actions include issuing guidance for rulewriters, ${ }^{433}$ increasing public participation for EPA-issued permits, building environmental justice into enforcement targeting, and the development of EJSCREEN ${ }^{434}$ and EJ Legal Tools. ${ }^{435}$

The Mercury and Air Toxics Standard (MATS) provides an example of how EPA incorporates EJ considerations in the rulemaking process. EPA used data on subsistence fishing and potential health impacts of mercury deposition on the minority, low-income and indigenous populations engaged in subsistence fishing in the initial finding that the rulemaking was "appropriate and necessary." In addition, EPA held a series of webinars, community calls, and consultations with tribal leadership to engage potentially affected populations in the rule-making process. The final regulatory impact analysis found the MATS rule will improve public health by lowering mercury exposure, especially for children and the elderly and for low-income, minority and indigenous populations that rely on subsistence fishing. ${ }^{436}$

Additionally, EPA has engaged in research and analytic efforts related to environmental justice that have resulted in better metrics and tools for understanding and analyzing EJ issues. A recent example is the rollout of EJScreen, a mapping tool that combines environmental and demographic data in maps and reports. Environmental indicators typically are direct or proxy estimates of risk, pollution levels, or potential exposure (e.g., due to nearby facilities). Demographic indicators are often used as proxies for a community's health status and potential susceptibility to pollution. This information can be used to highlight geographic areas and to identify candidate regions for further review, analysis or outreach. Screening tools such as EJScreen can provide environmental information to the public, and have also been used in aspects of enforcement, compliance, the Superfund program, permitting, and voluntary programs. ${ }^{437}$ EPA is also publishing a suite of resources designed to assist program implementers in bringing efficiency and renewable energy to low-income communities. These resources include a guide to EPA Programs for Low-Income Communities, a webcast series, and a series of program case studies highlighting best practices. ${ }^{\text {ddd }}$

DOE and other federal agencies also incorporate EJ considerations in agency activities and decision-making, e.g., in environmental impact statements in accordance with National Environmental Policy Act (NEPA) review. For example, in 2015 DOE completed 14 EISs, eee and each of these documents explicitly evaluated potential EJ impacts. In each case, the alternative that DOE ultimately selected will result in no disproportionately high and adverse human health or environmental effects on minority populations, low-income populations, or Indian tribes.

\footnotetext{
ddd See https://www.epa.gov/statelocalclimate/bringing-benefits-energy-efficiency-and-renewable-energy-lowincome-communities to access EPA resources.

eee See http://energy.gov/nepa/ for a complete list of DOE NEPA documents.
} 
Additionally, DOE's Weatherization Assistance Program (WAP) addresses the energy needs of low-income families, including those who may reside in EJ communities. Since its founding in 1976, WAP has helped more than 7 million families reduce their energy bills through costeffective energy efficiency measures, including those that encompass the building envelope, heating and cooling systems, electrical systems, and electricity consuming appliances. WAP services low-income families free of charge at an average expenditure of $\$ 6,800$ per residence, yielding a value of 2.9 times greater than the cost of weatherization improvements. ${ }^{438}$

Other DOE initiatives include: Tribal Energy Projects aimed at developing renewable energy projects on tribal lands to improve possibilities for economic development; ${ }^{439}$ energy efficiency standards for appliances and equipment, which reduce end-use energy demand; and increased community engagement and outreach through the national labs. ${ }^{440}$ 


\section{CONCLUSIONS}

\subsection{KEY FINDINGS}

This report reviews the recent literature that assesses a subset of the environmental impacts of the U.S electricity system. The primary focus is the air pollution impacts of power generation, arguably the source of the largest environmental impacts of the U.S. electricity system. The report also includes an assessment of land use impacts on the environment, water quality and environmental justice issues. The report does not address the issue of water use by the power sector, greenhouse gas emissions or solid waste from power generation because those topics are covered by other environmental baseline reports.

CAPs and HAPs are found in relatively large quantities in the lower atmosphere, particularly in populated, urban areas, where they pose risks to human health and the environment across broad regions of the country. However, air quality in the U.S. has been improving for decades and additional improvements are expected in the coming years, as a result of existing and expected market shifts, new regulations and technology improvements.

The U.S. power generation mix has rapidly evolved over the past several years. Of particular relevance to air quality, between 2001 and 2015, the contribution of coal-fired power to the total electric generation mix declined from $51 \%$ to $34 \%$. Natural gas power generation has steadily increased and surpassed coal power generation for an entire month for the first time in history, in 2015. The share of total power generation from wind and solar has increased from $1 \%$ to $4 \%$ from 2001 to 2014 and they are the fastest growing power generating technologies.

\subsubsection{Air Quality}

- The power sector is responsible for $64 \%$ of economy-wide $\mathrm{SO}_{2}$ emissions, $14 \%$ of $\mathrm{NO}_{\mathrm{x}}$ emissions, 3.4\% of $\mathrm{PM}_{2.5}$ emissions, and $1.4 \%$ of $\mathrm{PM}_{10}$ emissions.

- Among sources that are not related to land use (i.e., dust, agriculture and fires), the power sector accounts for $11 \%$ and $12 \% \mathrm{PM}_{10}$ and $\mathrm{PM}_{2.5}$ emissions, respectively.

- Within the power sector, coal accounts for $98 \%$ of $\mathrm{SO}_{2}$ emissions, $94 \%$ of mercury emissions, $86 \%$ of $\mathrm{NO}_{\mathrm{x}}$ emissions, and $83 \%$ of $\mathrm{PM}_{2.5}$ emissions. Within the power sector, natural gas accounts for $8 \%$ of $\mathrm{NO}_{\mathrm{x}}$ emissions, and $12 \%$ of $\mathrm{PM}_{2.5}$ emissions.

- Sulfur dioxide and NOx emissions from the U.S. electric power sector have declined sharply in recent decades, even as electricity generation has increased (Figure 6.1): 
- $2014 \mathrm{SO}_{2}$ emissions are 73\% lower than in 1970; 2014 NOx emissions are 57\% lower than $1970 \mathrm{NO}_{\mathrm{x}}$ emissions. ${ }^{441}$ Reductions in $\mathrm{SO}_{2}$ and $\mathrm{NO}_{\mathrm{x}}$ emissions increased rapidly after 1990.442443

- Emissions of $\mathrm{PM}_{2.5}$ and $\mathrm{PM}_{10}$ have declined by about $60 \%$ during the past ten years, in line with recent trends of $\mathrm{SO}_{2}$ and $\mathrm{NO}_{x}$ emissions.

Figure 6.1. Trend of selected criteria air pollutant emissions from fuel combustion in electric utilities. $^{444} 445$

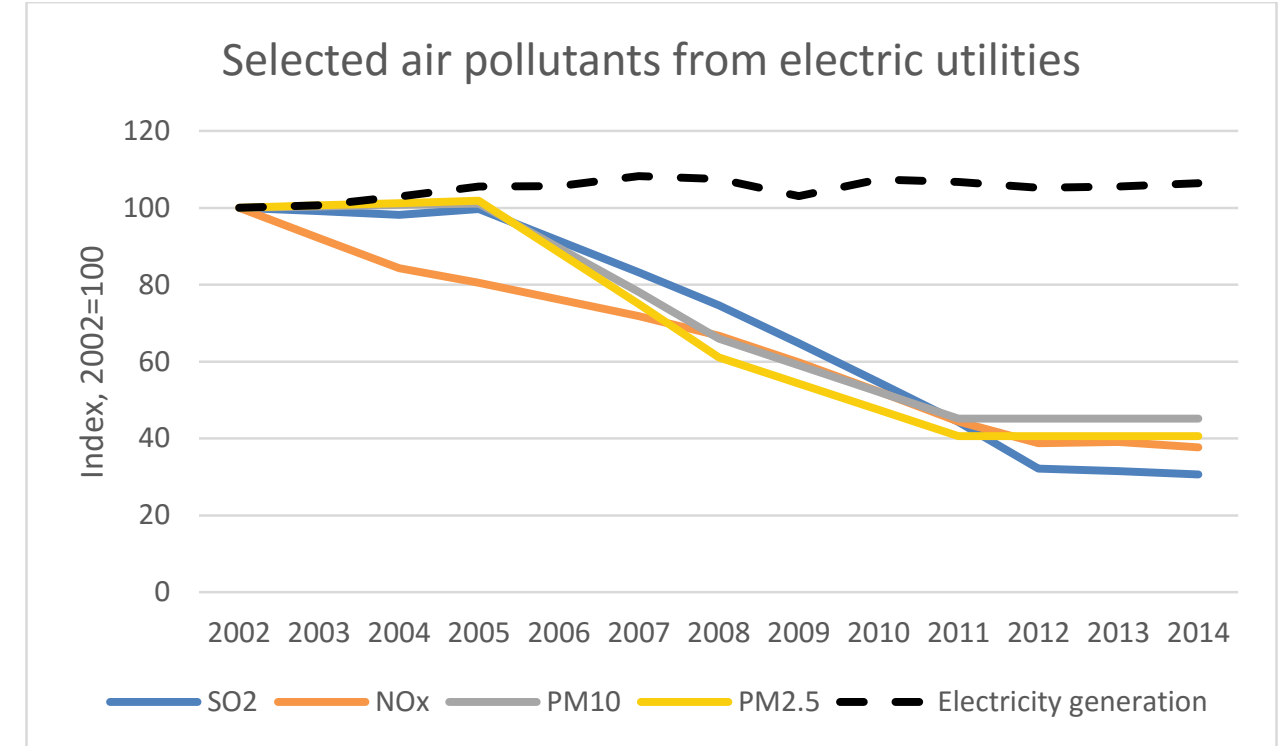

Power sector emissions of particulate matter, sulfur dioxide, and nitrogen oxides have declined in the range of 55 to 65 percent since 2002 .

- The primary factors affecting $\mathrm{SO}_{2}$ emissions from coal-fired power plants ${ }^{\mathrm{fff}}$ include the sulfur content of coal, total generation from coal, and the fraction of coal-fired power plants using flue gas desulfurization (FGD) pollution controls. The factors contributing to $\mathrm{SO}_{2}$ emission reductions have evolved over time (Figure 6.2).

- From 1994 to 2014, the one factor that has consistently been the biggest contributor to reductions in $\mathrm{SO}_{2}$ emissions is the installations of FGD controls, which were installed to comply with environmental regulations.

- From 1994 to 2004, the second biggest driver was the reduced sulfur content of the coal burned at power plants in the United States.

- From 2004 to 2014, the second biggest driver was a reduction in the use of coal and a shift toward the greater use of natural gas and renewables.

\footnotetext{
fff Coal-fired power plants are the predominant source of $\mathrm{SO}_{2}$ emissions from electric utilities and have been responsible for more than 95 percent of $\mathrm{SO}_{2}$ emissions reductions since 1989. As such, this analysis focuses on changes in $\mathrm{SO}_{2}$ emissions from coal-fired power plants.
} 
Figure 6.2. Factors Contributing to the Reduction in $\mathrm{SO}_{2}$ Emissions from Coal-Fired Power Plants: 1994-2014446 447448

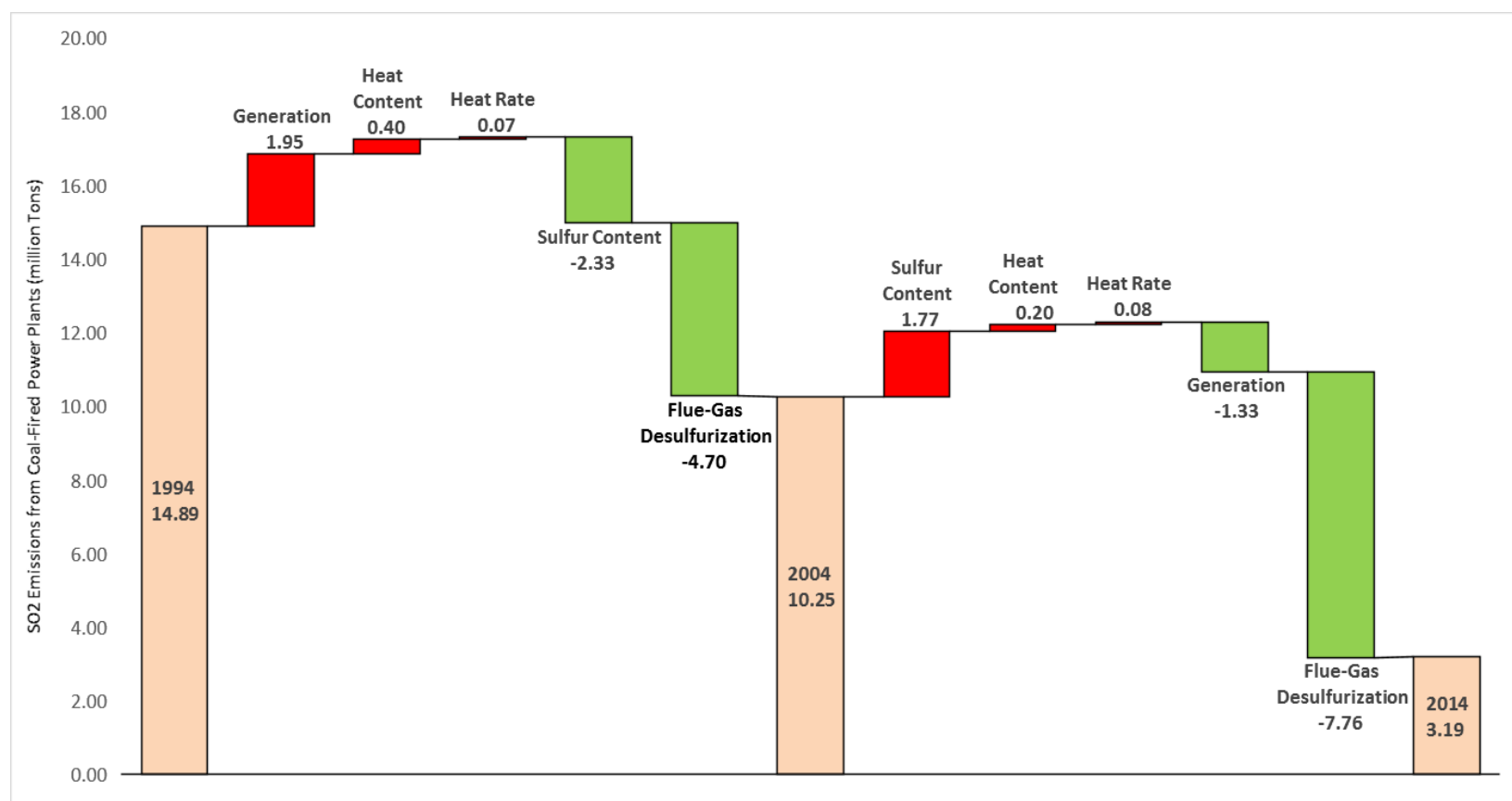

From 1994 to 2014, annual $\mathrm{SO}_{2}$ emissions from electric utilities have decreased by 11.7 million tons. Primary factors affecting $\mathrm{SO}_{2}$ emissions from coal-fired power plants include the sulfur content of coal, the heat content of coal, the heat rate of electricity generation, total generation from coal, and the fraction of coal-fired power plants using flue gas desulfurization (FGD) pollution controls.

Notes: The graph displays the contribution of different drivers to the trend of $\mathrm{SO}_{2}$ emissions. The vertical difference between the vertical bars that describe emissions in 1994, 2004 and 2014 is decomposed in different factors that affect emissions. The red bars indicate factors that have contributed to the increase of emissions and the green bars factors that have contributed to the decline of emissions, ceteris paribus. The contribution of each driver to the change of $\mathrm{SO}_{2}$ emissions is calculated assuming that all the other drivers remain constant during the time period being examined.

- Nearly all $\mathrm{SO}_{2}$ emissions from power generation are released by coal fired power plants, which are concentrated in the Eastern United States (Figure 6.3, panel A). NOx and PM emissions are more evenly distributed (Figure 6.3, panels B, C and D) as they are also released in significant quantities by natural gas-fired power plants. 
Figure 6.3. Map of 2011 emissions from power plants of $\mathrm{SO}_{2}, \mathrm{NO}_{x}$ and Particulate Matter. ${ }^{449}$

Sulfur Dioxide

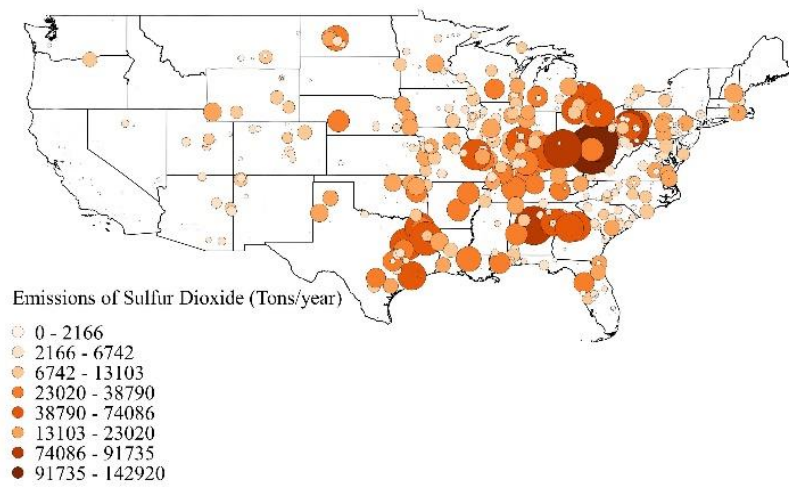

Nitrogen Oxides

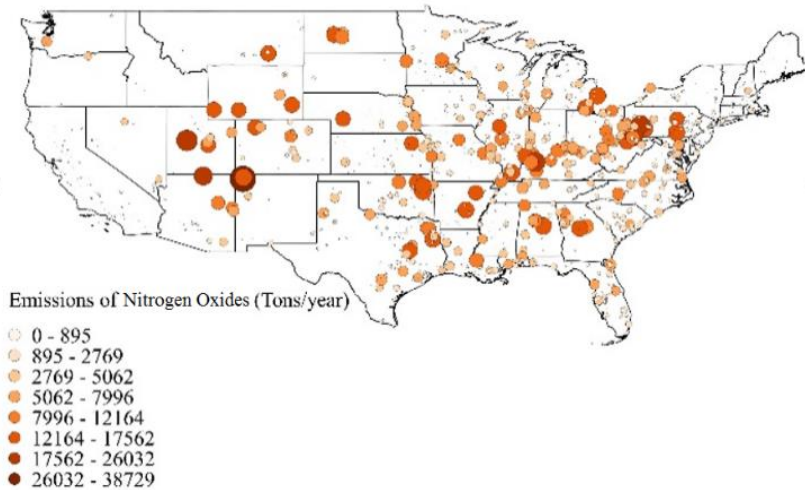

PM 2.5

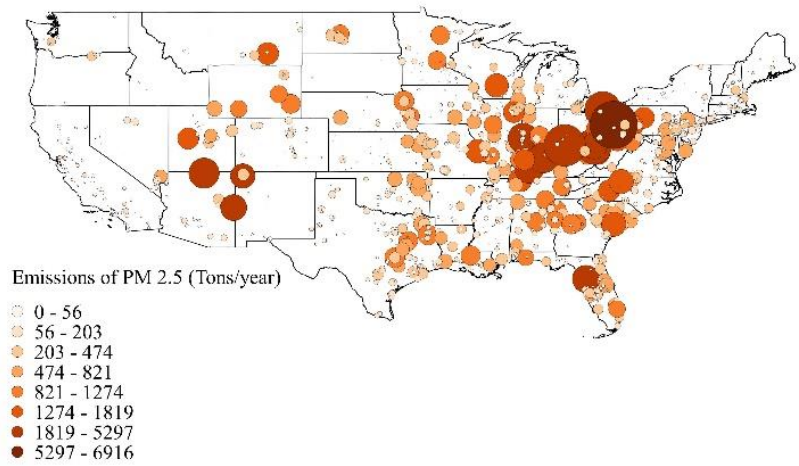

PM 10

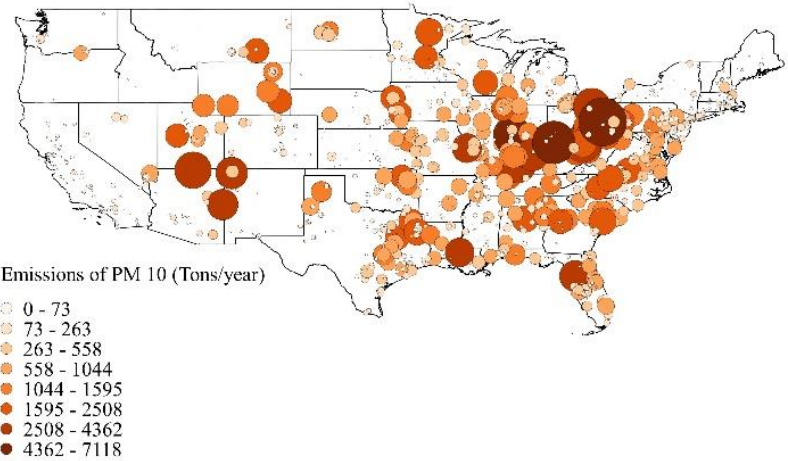

Virtually all $\mathrm{SO}_{2}$ is emitted from coal fired power plants—both with and without pollution controls -in the eastern United States. NOx and PM emissions are more evenly distributed, as they are also released in significant quantities by natural gas power plants.

- Recent studies estimate that the cost of air pollution from power generation in the United States, during the years 2002-2011, ranged between \$71 and \$223 billion per year. ${ }^{450451}$ 452453 Pollutants emitted by the electric power sector cause:

- Damage to human health (increased morbidity and mortality), to crops and timber production (productivity losses), and to materials (deterioration and increased maintenance costs).

- Reduced visibility and harm to ecosystems, with losses to recreational value and ecological services.

- Increased morbidity and mortality are responsible for the largest share of external costs of power generation. ${ }^{454} 455456457458$ 
- Human health damages associated with exposure to $\mathrm{PM}_{2.5}$ constitute the largest source of economic losses associated with air pollution. 459460461462

- One of the main reasons behind the large difference in pollution cost estimates in the literature is that different studies use different assumptions to monetize the value of increased mortality risk due to pollution.

- The actual benefit per ton of reducing all non-GHG air pollutants varies depending on the location of the emission reduction, with emissions reductions in urban areas resulting in greater benefits per ton of abatement.

- Clean Air Act (CAA) regulations help to reduce air pollution from the power sector.

- Under the National Ambient Air Quality Standards (NAAQS) provisions of the CAA, EPA is required to set standards for CAPs and to periodically review the standards to ensure that they are based on current science. Current NAAQS for ozone were finalized in 2015. Current NAAQS for PM were finalized in 2012.

- Under section 126 of the CAA, EPA has issued the Cross-State Air Pollution Rule to cut interstate pollution of $\mathrm{NO}_{x}$ and $\mathrm{SO}_{2}$ in the eastern U.S.

- Under the National Emission Standards for Hazardous Air Pollutants (NESHAP) provisions of the CAA, EPA sets standards for emissions of HAPs (like mercury). In 2011, EPA finalized the Mercury and Air Toxics Standards (MATS) that establishes toxic emissions limits for new and existing coal- and oil-fired power plants.

- As a result of CAA regulations, nearly all coal-fired power plants in the United States have installed controls for emissions of $\mathrm{NO}_{x}$ and $\mathrm{SO}_{2}$. Emissions of $\mathrm{SO}_{2}$ and of mercury are expected to sharply decline in $\mathbf{2 0 1 6}$ as all affected sources are expected to be in full compliance with MATS.

- The U.S. Acid Rain Program (ARP) is broadly recognized as a success story in pollution regulation, with near-perfect compliance and lower than expected costs. ${ }^{463}$

- The ARP achieved more than a 70 percent reduction in powerplant $\mathrm{SO}_{2}$ emissions since $1990^{464}$ and the resulting health and environmental benefits are much larger and costs much smaller than originally estimated. ${ }^{465}$ Costs have been lower than expected due to advancements in technology and - particularly in the 1990s - lower prices for low-sulfur coal. ${ }^{466}$

- Estimates of health benefits vary but are considered to be around \$50 billion per year, in $2010 .{ }^{467}$ Improved health conditions and lower risk of mortality are the dominant benefits of reducing emissions. ${ }^{468}$

- $\quad$ Estimates of ARP compliance costs have ranged from $\$ 0.5$ to $\$ 5.3$ billion per year. 
- Emissions trading under the ARP has greatly reduced compliance costs in general by allowing flexibility for the industry to achieve emission reductions at power plants with the most cost-effective pollution control opportunities. ${ }^{469}$

\subsubsection{Land and Ecosystem Impacts}

- A key finding from the first installment of the QER is that while expanding transmission and distribution infrastructure can pose environmental challenges, building new infrastructure can help to enable significant net positive environmental outcomes. ${ }^{470}$ For this reason, agencies across the federal government are engaged in several initiatives to modernize the Federal Governments role with respect to electric transmission permitting and project review. ${ }^{471}$ These initiatives (Table 3.3) are designed to support better decision making - while enabling better outcomes for public health, the environment, safety and communities - in the following ways:

- Facilitate better coordination between permitting authorities at all levels of government,

- Develop and publish relevant information, data and tools,

- Support infrastructure planning; establish rights of way for energy projects, and

- Conduct technology research and development.

- There is very limited published literature comparing life cycle land use impacts across all electricity generation technologies. ${ }^{472}$ Land use requirements for renewable energy are dominated by the operational footprint of the generation facilities; for non-renewable power generation options, the opposite is generally true, when the upstream impacts of fuel resource extraction are accounted for. One study ${ }^{473}$ normalized land requirements during the life cycles of conventional- and renewable-energy options and found that:

- The PV cycle requires the least amount of land among renewable-energy options, while the biomass cycle requires the largest amount.

- The land requirements of ground-mounted PV systems in areas with high quality solar resources is no greater than that of coal-based fuel cycles.

- Electricity Infrastructure and operations can be detrimental to wildlife in a number of ways; however, technical and operational solutions are under active development.

- Fish and birds are impacted by air and water pollution from power plants. Nuclear and fossil fuel-related bird deaths have been estimated to be comparable to deaths caused by wind power, per kWh generated. ${ }^{474}$

- Avian mortalities are caused by wind turbines and concentrated solar plants; impacts vary significantly by region and facility. However, bird mortalities due to collisions with 
wind turbines are much lower compared to bird mortalities from collisions with other engineered structures.

- There are several ongoing research efforts - many conducted in direct collaboration with industry and stakeholders - to better understand the environmental impacts of renewable energy facilities and to develop new technologies and operational practices for mitigating impacts.

- Herbicide treatment used for vegetation control along transmission rights of way can pollute surface and ground water and harm wildlife if done improperly. Some electric utilities are demonstrating more of a commitment to wildlife habitat and conservation when managing vegetation in the proximity of their transmission lines. For example, clearings for transmission lines serve as firebreaks, improve emergency access, and create meadows and edge habitats that can be managed in ways that are beneficial for certain species. ${ }^{475}$

\subsubsection{Water Quality}

- Steam electric power plants affect water quality through a variety of mechanisms.

- Air emissions of mercury and $\mathrm{SO}_{2}$ impact water quality through wet deposition.

- Additionally, steam electric plants generate wastewater streams that can impact ground water and surface water quality. Major waste streams from steam electric power plants include wastewaters from air pollution control systems, discharges associated with coal ash handling operations, coal pile runoff, condenser cooling, equipment cleaning, and leachate from landfills and impoundments. ${ }^{476}$

- Steam electric power plants are the largest source of toxic discharges, accounting for about 30 percent of all discharges from industrial sources, in toxic weighted pound equivalent (TWPE) units. ${ }^{477}$ Baseline discharges from steam electric power plants are estimated to be 2,680,000 lb-eq/yr, nearly three times the amount estimated for the pulp, paper, and paperboard industry, the petroleum refining industry, and nonferrous metals manufacturing industry (second, third, and fourth highest ranking).

- Wastewater pollutants include both toxic and bioaccumulative pollutants such as arsenic, lead, mercury, selenium, chromium, and cadmium. Wastewaters also contain significant levels of chloride, total dissolved solids (TDS), total suspended solids (TSS), and nutrients. Discharges of toxic bioaccumulative pollutants such as mercury, lead, and other heavy metals are of particular concern because they do not volatilize, do not biodegrade, can be toxic to plants, invertebrates and fish, adsorb to sediments, and bioconcentrate in fish tissues. ${ }^{478}$ 
- Impacts from these pollutants fall into several broad categories, including increased health risks, impacts to surface and ground water quality, and other environmental impacts. ${ }^{479}$

- Major impacts to human health from exposure to contaminated fish tissue include lowered IQ in children and infants from exposure to lead and mercury, increased incidence of cardiovascular disease in adults from exposure to lead and arsenic, and increased incidence of cancer from exposure to arsenic. ${ }^{480}$

- Water quality issues caused by electric power generation pose a particular risk for recreation and subsistence fishing communities, including indigenous peoples.

- Wastewater discharges can also impact groundwater and drinking water quality. Exceedances of maximum contaminant levels (MCLs) in drinking water downstream from power plants indicate an ongoing human health concern. In the environmental assessment for the steam electric effluent limitation guidelines, EPA identified more than 30 documented cases where groundwater contamination from surface impoundments extended beyond the plant boundaries. ${ }^{481}$

- Major policies regulating water quality impacts from steam electric power generation include the effluent limitations guidelines (ELGs) promulgated under the Clean Water Act, and the coal combustion residuals (CCR) disposal requirements promulgated under the Resource Conservation and Recovery Act.

- The new effluent limitation guidelines (September 2015) are projected to reduce pollutant loadings from existing sources by over 95 percent for copper, lead, mercury, nickel, selenium, thallium, and zinc and over 90 percent for arsenic and cadmium. Total TWPE discharges for key pollutants are projected to decline by 63 percent, from $2,680,000$ TWPE/year in the baseline to 1,000,000 TWPE/yr under the new effluent guidelines (Table 6.1). 
Table 6.1. Estimated Pollutant Loadings and Removals under the Steam Electric Effluent Limitation Guidelines (ELGs) ${ }^{482}$

\begin{tabular}{lcc} 
& \multicolumn{2}{c}{ Total Industry Loading } \\
& Pounds/Year & TWPE/Year \\
\hline Baseline & $2,210,000,000$ & $2,680,000$ \\
Pollutant removals under the rule* & $513,000,000$ & $1,680,000$ \\
Steam Electric ELGs & $1,700,000,000$ & $1,000,000$ \\
\hline
\end{tabular}

As the updated steam electric effluent limitations and guidelines (ELGs) go into effect, annual TWPE discharges are projected to decline by 63 percent, from 2,680,000 TWPE/year in the baseline to $1,000,000$ TWPE/year under the new regulations. Each steam electric plant must reach compliance between 2018 and 2023 depending on when it needs a new discharge permit.

Note: Pollutants accounted for in these estimates include only the pollutants listed in Table 4.2. The rule is also projected to reduce nitrogen loadings by 16.8 million lbs/yr (99 percent), phosphorus loadings by $174,000 \mathrm{lbs} / \mathrm{yr}$ (81 percent), and total dissolved solids (TDS) loadings by 1.32 billion lbs/yr (31 percent).

- $\quad$ The new CCR rule, promulgated in December 2014, addresses risks to groundwater quality from leaking impoundments. This rule establishes technical requirements for CCR surface impoundments, including composite liners, groundwater monitoring, corrective action, and closure/post closure care, among others. ${ }^{483}$

\subsubsection{Environmental Justice}

- Environmental justice (EJ) is the fair treatment and meaningful involvement of all people regardless of race, color, national origin, or income with respect to the development, implementation, and enforcement of environmental laws, regulations, and policies. ${ }^{484}$ Environmental justice is achieved when everyone enjoys the same degree of protection from environmental and health hazards and equal access to the decision-making process to have a healthy environment in which to live, learn, and work. ${ }^{485} 486$

- EJ concerns related to the electric power industry include the impacts to air quality and water quality, the impacts of climate change, and equal access to affordable energy and ratepayer impacts.

- Environmental justice communities are disproportionately exposed to air quality and water quality issues associated with electric power generation. Compared to the U.S. population overall, there is a greater concentration of minorities living within a three-mile radius of coal- and oil-fired power plants. In these same areas, the percent of the population below the poverty line is also higher than the national average. ${ }^{487}$

- Environmental justice communities are especially vulnerable to the impacts of climate change and also to some resilience strategies. Certain climate change related impacts- 
including heat waves, degraded air quality, and extreme weather events-have disproportionate effects on low-income populations and some communities of color. Existing health disparities and other inequities in these communities increase their vulnerability to the health effects of climate change.

- Federal agencies regularly address environmental justice considerations in their rulemakings and permitting processes. The responsibility of Federal agencies to consider environmental justice is set out in Executive Order No. $12898^{488} 489$ and typically implemented in conjunction with NEPA review, as described in the Council on Environmental Quality (CEQ) guidance for environmental justice. ${ }^{490}$

\subsubsection{Crosscutting}

- The price of electricity still does not reflect its full cost for the environment and society more broadly.

- Available literature on economic implications of energy infrastructure remains limited, particularly with respect to land and ecosystem impacts.

- There is also limited information and tools available on the demographics of populations exposed to environmental quality issues.

\subsection{ANALYSIS OPPORTUNITIES AND DATA LIMITATIONS}

The current understanding of environmental impacts is lacking in many key areas. Clearly, additional technical and socio-economic research would be useful for industry stakeholders, policy makers, and consumers. For example, the literature that tracks the effects of pollution from individual power plants is limited. The attribution of pollution damages to specific sources can only be done using sophisticated computational models. The environmental and economic impacts of mercury emissions could be better characterized. Tracking mercury across many ecosystems and monitoring its impact on human health is particularly challenging.

Few studies attempt to monetize the external costs associated with the net land-use and ecosystem impacts of the U.S. electricity system. More analysis of these and other issues is needed to provide a more comprehensive assessment of the true costs and benefits of electric power generation. 


\section{APPENDIX A. DECOMPOSITION ANALYSIS OF THE EVOLVING CONTRIBUTORS TO $\mathrm{SO}_{2}$ EMISSIONS REDUCTIONS IN THE U.S. ELECTRICITY SECTOR}

\section{INTRODUCTION}

$\mathrm{SO}_{2}$ emissions from electric utilities have decreased significantly over the past forty years. In 1975 they peaked at slightly more than 18 million tons and then declined almost monotonically to 3.2 million tons in 2014 (Figure A.1). In the period between 1994 and 2004, the focus of this decomposition analysis, $\mathrm{SO}_{2}$ emissions decreased by 11.7 million tons/year.

Figure A.1. 1970-2014 $\mathrm{SO}_{2}$ Emissions from Electric Utilities ${ }^{491} \mathrm{ggg}$

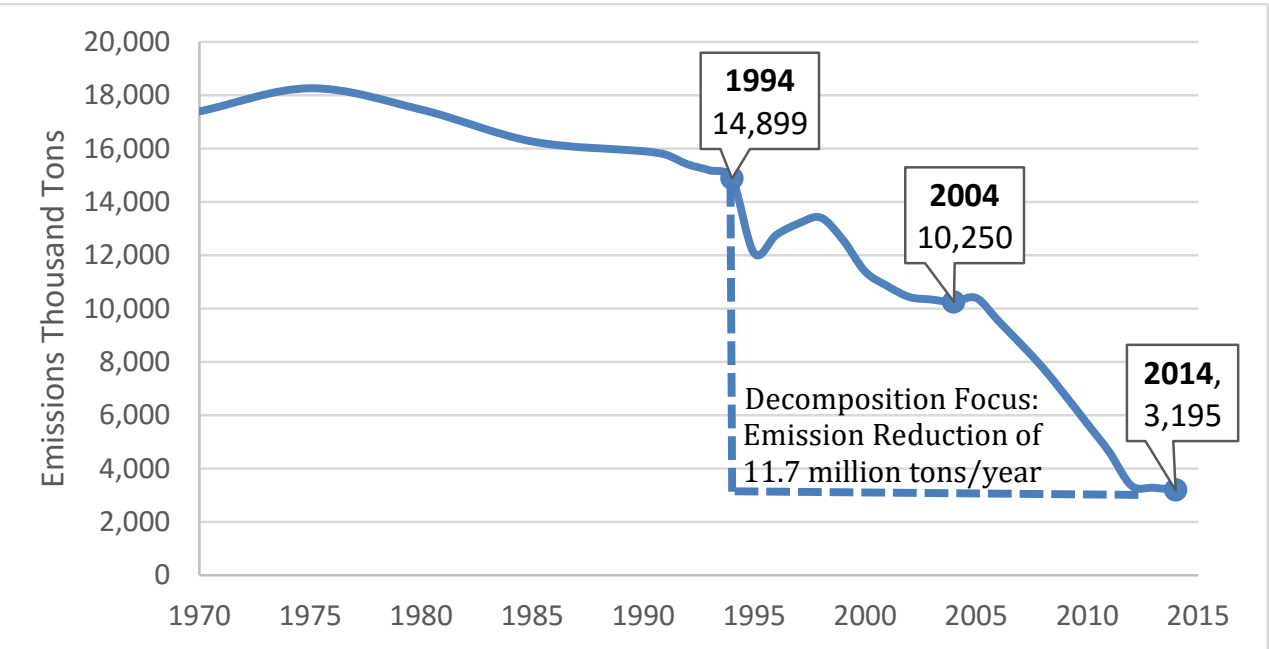

Over the twenty-year period from 1994 to 2014 , annual $\mathrm{SO}_{2}$ emissions fell by 11.7 million tons, a $79 \%$ decline.

The factors contributing to these reductions have evolved over time and the pace of emission reductions has been variable. While prior research has examined the impact of policies and markets on these trends, the influence of different contributing factors have changed in recent years. This appendix quantitatively evaluates five factors that have historically impacted $\mathrm{SO}_{2}$ emissions from coal-fired power plants, examining their influence over the past two decades using decomposition analysis.

Coal-fired power plants are the main source of $\mathrm{SO}_{2}$ emissions in the United States: from 1989 to 2011, more than $90 \%$ of total U.S. $\mathrm{SO}_{2}$ emissions were from coal plants. ${ }^{492}$ In addition, coal

ggg Prior to 1994, $\mathrm{SO}_{2}$ emissions were estimated rather than aggregated from facility-level reporting. According to Title IV (Acid Deposition Control) of the Clean Air Act Amendments of 1990 (CAAA)ggg published in January 1994, utility units are required to report $\mathrm{SO}_{2}$ data to EPA. Beginning January 1,1995 , all affected units were required to report heat input and $\mathrm{SO}_{2}$ emissions. This change contributed to the "kink" in the curve between 1994 and 1995. 
plants have been responsible for more than $95 \%$ of $\mathrm{SO}_{2}$ emission reductions since 1989 . Being the dominant source of $\mathrm{SO}_{2}$ emissions, the following analysis focuses on the changes in $\mathrm{SO}_{2}$ emission from coal-fired power plants.

\section{METHODOLOGY AND DATA SOURCES}

The decomposition method used in this study is similar to the expanded Kaya identity that was applied to $\mathrm{CO}_{2}$ emissions and its contributing factors. The Kaya identity, initially proposed in 1990 at an IPCC workshop, correlates the quantitative relationship between national $\mathrm{CO}_{2}$ emissions and its contributing factors: energy intensity, economic growth (often characterized as Gross Domestic Products) and population growth. ${ }^{493}$ Since then, decomposition analysis has become a commonly accepted method widely used in IPCC reports and other academic discussions of carbon emission and its contributing factors. ${ }^{494}$ Using the same approach, the attribution of $\mathrm{SO} 2$ emissions reductions to different drivers can be evaluated as follows:

$$
\mathrm{SO}_{2}=\left(\frac{\mathrm{SO}_{2}}{S}\right) *\left[\left(\frac{S}{\text { Coal }}\right) /\left(\frac{E_{\text {coal }}}{\text { Coal }}\right)\right] *\left(\frac{E_{\text {coal }}}{G}\right) * G *(1-F G D)
$$

In simplified symbols,

$$
S_{t}=1.998 * s_{t} / c_{t} * h_{t} * g_{t} *\left(1-f_{t}\right)
$$

Specifically, $\mathrm{SO}_{2}$ emissions are decomposed using the following factors: the combustion factor for sulfur $\left(\mathrm{SO}_{2} / \mathrm{S}\right)$, the sulfur content of coal (S/Coal), the heat content of coal (Ecoal/Coal), the heat rate of coal (Ecoal/G), total power generation (G), and the fraction of emissions after flue gas desulfurization (FGD). These different components are described in greater detail in Table A.1. This decomposition allows for attribution of the change in $\mathrm{SO}_{2}$ emissions to each component driver and the factors contributing most to the decline in $\mathrm{SO}_{2}$ emissions.

$\mathrm{SO}_{2} / \mathrm{S}$ is also called the sulfur retention ratio. It is a function of the fraction of sulfur in the coal that ends up in coal ash after combustion and the relative atomic weight of $\mathrm{SO}_{2}$ to $\mathrm{S}$. The former varies from $0 \%$ to about $10 \%$ resulting from the varying composition and quality of coal, along with the operational conditions of plants. 495496 In the absence of accurate consensus data, we here approximately assume that all sulfur goes into sulfur emissions after combustion. In other words, we use the relative weight of $\mathrm{SO}_{2}$ to $\mathrm{S}, 1.998$, to represent the $\mathrm{SO}_{2} / \mathrm{S}$ value. Because this value is assumed to be constant over time, it is not studied further as a contributing factor to $\mathrm{SO}_{2}$ emission reductions. 
Table A.1. Contributing Factors, Units and Data Sources

\begin{tabular}{|c|c|c|c|c|c|}
\hline Factor & $\begin{array}{l}\text { Simplified } \\
\text { Symbol }\end{array}$ & Explanation & Unit & Source & Figure \\
\hline S/Coal & St & $\begin{array}{l}\text { Sulfur Content of } \\
\text { Coal Used }\end{array}$ & $\begin{array}{l}\% \text { in } \\
\text { Weight }\end{array}$ & $\begin{array}{l}\text { EIA-923 Survey, } \\
\text { https://www.eia.gov/electricity/data/eia923/ }\end{array}$ & A. 2 \\
\hline E/Coal & $\mathrm{Ct}_{\mathrm{t}}$ & $\begin{array}{l}\text { Heat Content of } \\
\text { Coal used }\end{array}$ & Btu/tons & $\begin{array}{l}\text { EIA Cost and Quality of Fuels for Electric Plants } \\
\text { Report }\end{array}$ & A. 3 \\
\hline$E / G$ & $h_{t}$ & $\begin{array}{l}\text { Heat Rate of Coal } \\
\text { Plants }\end{array}$ & $\begin{array}{l}\text { Btu/tons } \\
\text { coal }\end{array}$ & $\begin{array}{l}\text { EIA Monthly Energy Review } \\
\text { http://www.eia.gov/totalenergy/data/monthly/ }\end{array}$ & A.7 \\
\hline G & $g_{t}$ & $\begin{array}{l}\text { Total Generation } \\
\text { from Coal Plants }\end{array}$ & MWh & $\begin{array}{l}\text { EIA Monthly Energy Review } \\
\text { http://www.eia.gov/totalenergy/data/monthly/ }\end{array}$ & A. 8 \\
\hline FGD & $f_{t}$ & $\begin{array}{l}\text { Fraction of } \\
\text { Emissions Lacking } \\
\text { Flue Gas } \\
\text { Desulfurization }\end{array}$ & $\%$ & $\begin{array}{l}\text { EIA Annual Electric Generator Report, } \\
\text { https://www.eia.gov/electricity/data/eia860/ } \\
\text { Calculated Given Other Variables }\end{array}$ & A. 10 \\
\hline
\end{tabular}

Using the data from the sources in Table A.1, we identify and characterize the relationship of the five factors to the emissions reduction of $\mathrm{SO}_{2}$. To illustrate how we calculate the magnitude of a factor's contribution, consider the 1994-2014 period. Using the natural log of the change in emissions over these two decades simplifies the analysis:

$$
\ln \left(\frac{S_{2014}}{S_{1994}}\right)=\ln \left(\frac{S_{2014}}{S_{1994}}\right)+\ln \left(\frac{c_{2014}}{c_{1994}}\right)+\ln \left(\frac{h_{2014}}{h_{1994}}\right)+\ln \left(\frac{g_{2014}}{g_{1994}}\right)+\ln \left(\frac{f_{2014}}{f_{1994}}\right)
$$

From here, the function is multiplied by the factor required on both sides to set the left side equal to $\Delta S$.

$$
\Delta S=\frac{\Delta S}{\ln \left(S_{2014} / S_{1994}\right)}\left[\ln \left(\frac{S_{2014}}{S_{1994}}\right)+\ln \left(\frac{c_{2014}}{c_{1994}}\right)+\ln \left(\frac{h_{2014}}{h_{1994}}\right)+\ln \left(\frac{g_{2014}}{g_{1994}}\right)+\ln \left(\frac{f_{2014}}{f_{1994}}\right)\right]
$$

Each of the terms on the right hand side is the estimated amount of emissions reduction resulting from that particular factor. Each factor's contribution is calculated as:

$$
\frac{\Delta S}{\ln \left(S_{2014} / S_{1994}\right)} \ln \left(\frac{F_{2014}}{F_{1994}}\right)=\text { emissions reduction resulting from a change in factor } F
$$

\section{CONTRIBUTING FACTORS AND TRENDS OVER THE PAST 20 YEARS}

From the above equation, the contributing factors that determine $\mathrm{SO}_{2}$ emissions can be easily characterized. The five factors are sulfur content of coal, heat content, heat rate, total generation form coal and the application of FGD. With these factors, the puzzle of $\mathrm{SO}_{2}$ emissions reduction can be solved by illustrating the change of each factor. 
These five factors can be divided into three categories: coal quality (sulfur content and heat content), plant performance (heat rate and generation) and emission control (desulfurization technology). We now examine the trend of each factor, by category.

\subsection{COAL QUALITY: SULFUR CONTENT AND HEAT CONTENT}

The sulfur content of coal since 1994 is shown in Figure A.2. Specifically, the sulfur content of the coal used in electric utilities in the past 20 years has increased from less than 1.2 to about 1.3 percentage, by weight. Thus, coal plants in 2014 used higher sulfur coal to generate electricity in 1994. On the other hand, the heat content of coal used in the past 20 years indicates that this feature of coal resources declined between 1994 and 2014. Figure A.3 indicates that the heat content of coal has slightly decreased from above 20 million Btu/ton coal in 1994 to slightly less than 20 million Btu/ton in 2014.

\section{Figure A.2. 1994-2014 Sulfur Content of Coal used in Electric Utilities (Left) ${ }^{497}$} Figure A.3. 1994-2014 Heat Content of Coal used in Electric Utilities (Right) ${ }^{498} 499$
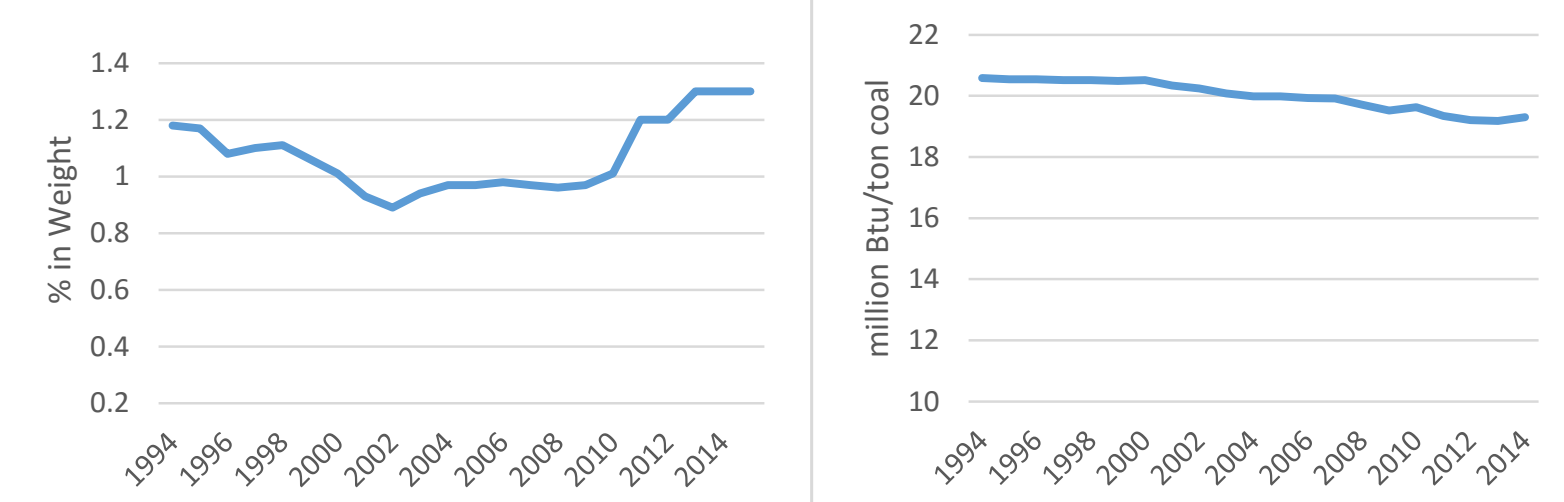

Over the last twenty years, the sulfur content of coal has fluctuated between $0.9 \%$ and $1.3 \%$, by weight. The heat content of coal has been on a steady, gradual decline.

To better understand these two factors, we need to examine coal quality since these two factors are bundled together when power plants purchase and consume coal. To do this, we introduce the concepts of coal rank and quality. In the U.S., the heat content of coal is highest in Anthracite and Bituminous, and is lowest in Subbituminous and Lignite. Anthracite is rare in production and consumption, so the other three types of coal are often referred to as the main coal ranks in the North American. The main production regions are shown in Figure A.4. In general, specific types of coal can be found concentrated in specific areas.

- Anthracite - northeastern Pennsylvania

- Bituminous - east of the Mississippi River, with the greatest amounts in Illinois, Kentucky, and West Virginia; 
- Subbituminous coal - west of the Mississippi River, with the greatest amounts in Montana and Wyoming

- Lignite - Montana, Texas, and North Dakota.

Figure A.4. Coal-Bearing Areas of the United States ${ }^{500}$

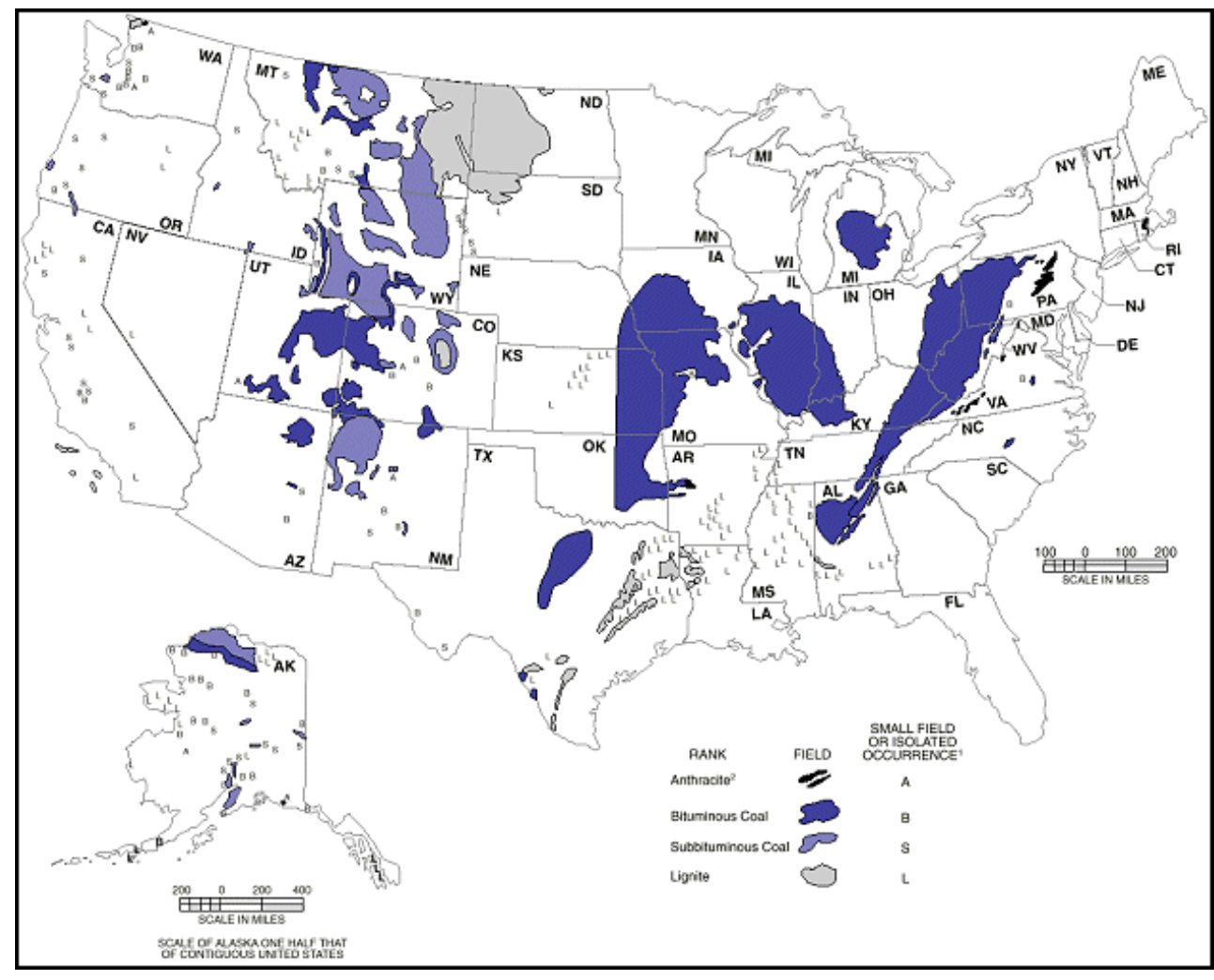

Bituminous, subbituminous, and lignite coal production in the United States. In general, bituminous coal has the highest sulfur content. 
Figure A.5. Coal Production by Region, Year and Coal Rank ${ }^{501}$

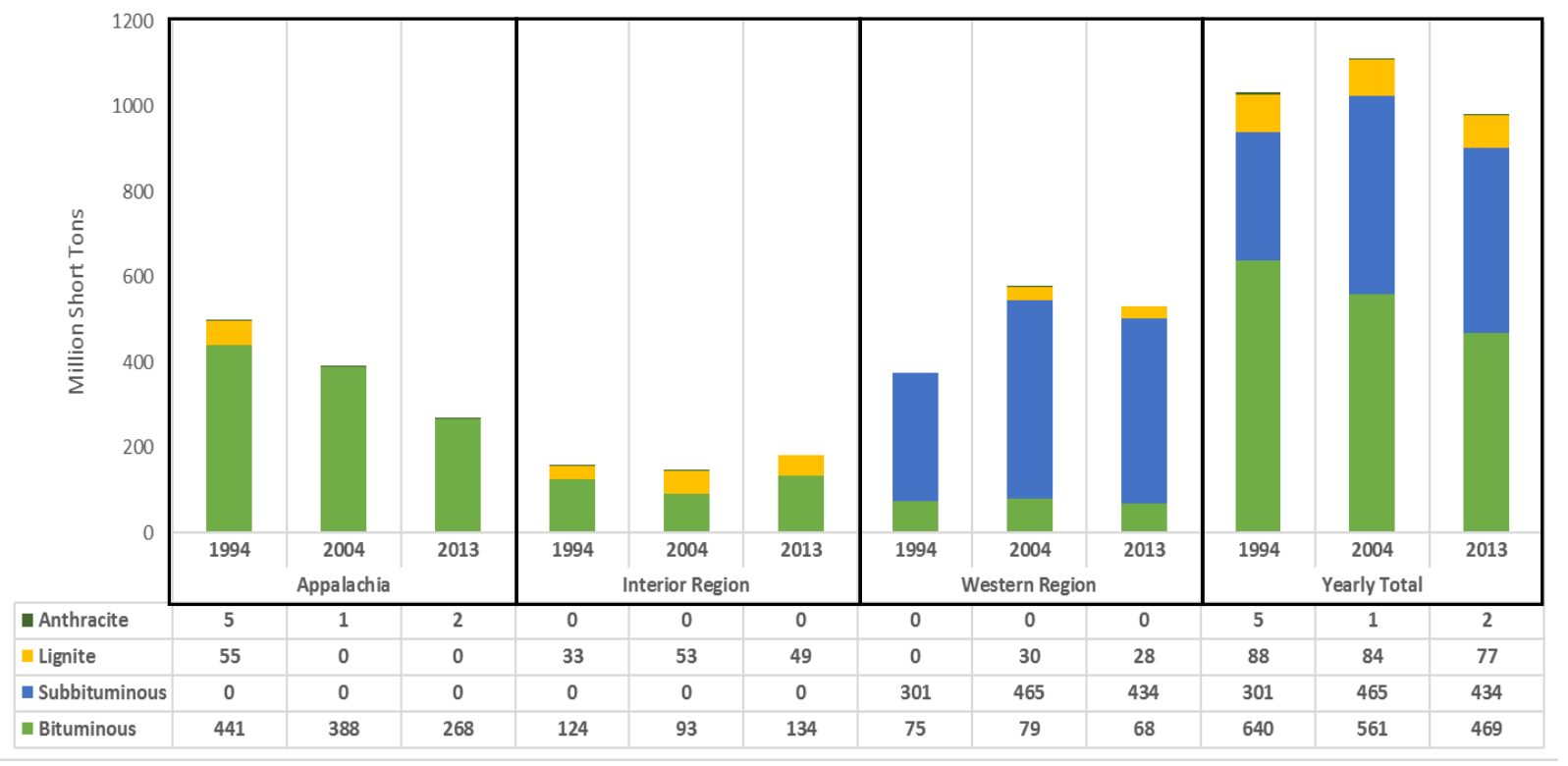

From 1994 to 2004, U.S. coal production shifted from Appalachia bituminous coal to Western subbituminous coal, contributing to lower overall sulfur content in the coal used for electricity generation. From 2004 to 2013, production of bituminous coal continued to decline; however, this decline was offset by an increase in the sulfur content bituminous coal (Table A.2).

Figure A.5 describes the level and types of total U.S. coal production in 1994, 2004 and 2014 by region and coal rank. Note that this is the total coal generation from U.S. domestic coal mines, a portion of which is consumed by the electric power sector. Since coal used by the power sector as a percentage of total production was $89 \%$ in $1994,91 \%$ in $2004,92 \%$ in 2014 , it is reasonable to explain the shift using the total generation data available. This figure clearly shows that in the first decade of our analysis period, U.S. coal production shifted from Appalachia bituminous to Western subbituminous. Since bituminous coal has greater sulfur content than subbituminous, this shift contributed to $\mathrm{SO}_{2}$ emission reductions. Between 2004 and 2014, the nation experienced an increase in the production of bituminous coal from mines in the Interior region. Appalachia production continued to decrease in this second decade, but less than during the first 10 years, while Western region production decreased slightly.

Over the same period, the quality of different ranks of coal changed, especially bituminous (Table A.2). Specifically, the sulfur content of bituminous increased from 1.5\% in 2004 to $2.3 \%$ by weight in 2014 , driving the total change from $0.97 \%$ to $1.3 \%$. This rising sulfur content reduced the pace of $\mathrm{SO}_{2}$ emission reductions over the most recent decade. 
Table A.2. Sulfur Content of Coal Used in the Electric Sector, by Rank 502503

\begin{tabular}{lcc}
\hline \% in Weight & 2004* & $\mathbf{2 0 1 4}$ \\
\hline All Rank Average & 0.97 & 1.3 \\
Bituminous & 1.5 & 2.3 \\
Subbituminous & 0.36 & 0.3 \\
Lignite & 1.05 & 0.9 \\
\hline
\end{tabular}

* Data is not available for 1994.

At the same time that the sulfur content of bituminous coal was increasing, the price of coal was also on the rise (Figure A.6). While the cost of coal decreased from 1994 to 2004, it increased from 2004 to 2014. The high price for bituminous coal may have caused power plants to move to coal with lower sulfur content, which was also cheaper. The Clean Air Act may also have been a factor. The causal relationship can only be presumed, and cannot be tested using a decomposition approach.

Figure A.6. The Cost of Coal Consumed in the Electric Sector ${ }^{504}$

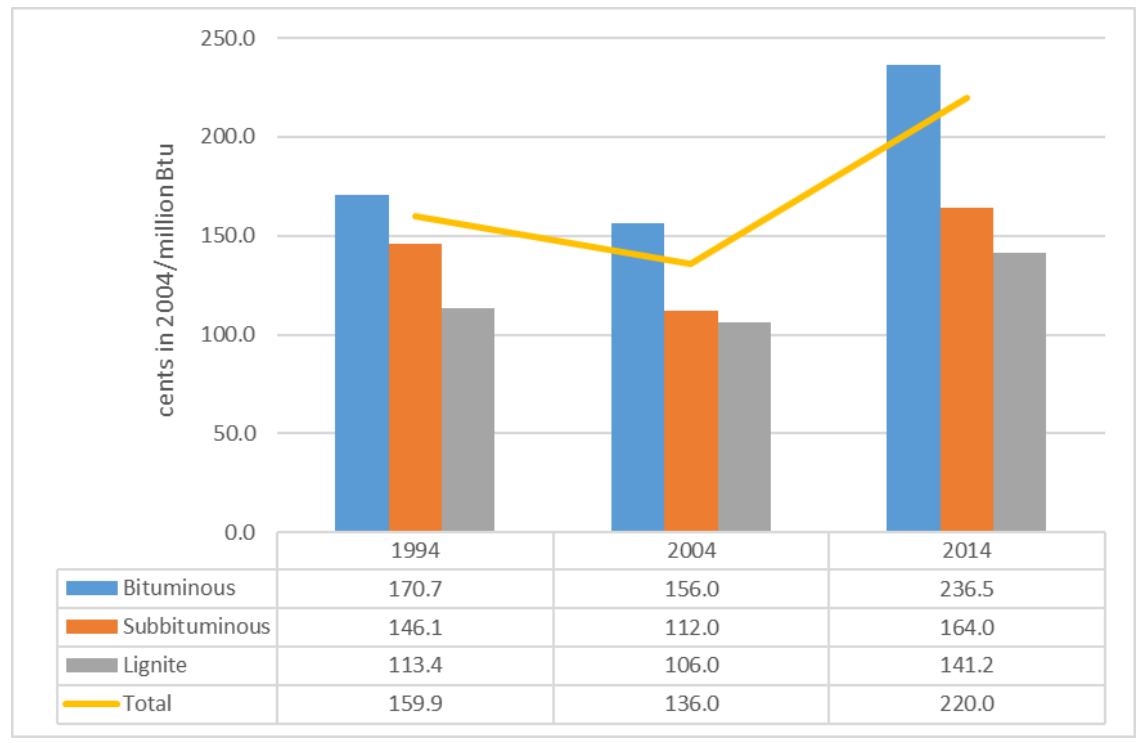

The cost of coal across all ranks declined from 1994 to 2004, and increased from 2004 to 2014 .

\subsection{PLANT PERFORMANCES: HEAT RATE AND ELECTRICITY GENERATION}

The national heat rate of coal plants has increased slightly over the past two decades, rising from 10.2 thousand Btu/kWh in 1994 to about 10.4 thousand Btu/kWh in 2014 (Figure A.7). Thus, coal plants are operating about $2 \%$ less efficiently today compared with 20 years ago. This trend is reasonable considering the fact that electricity generation technologies used by coal plants are mature and stable. Other contributors are the implementation of environmental control equipment, existing power plants degradation with relatively few new plants, and the 
possible shift of load into less efficient coal plants. Since the change in heat rate is relatively small, the driving reasons are difficult to identify.

Figure A.7. Heat Rate of Coal Plants 1994-2014 (Left) ${ }^{505}$

Figure A.8. Total Generation from Coal Plants 1994-2014 (Right) ${ }^{506}$
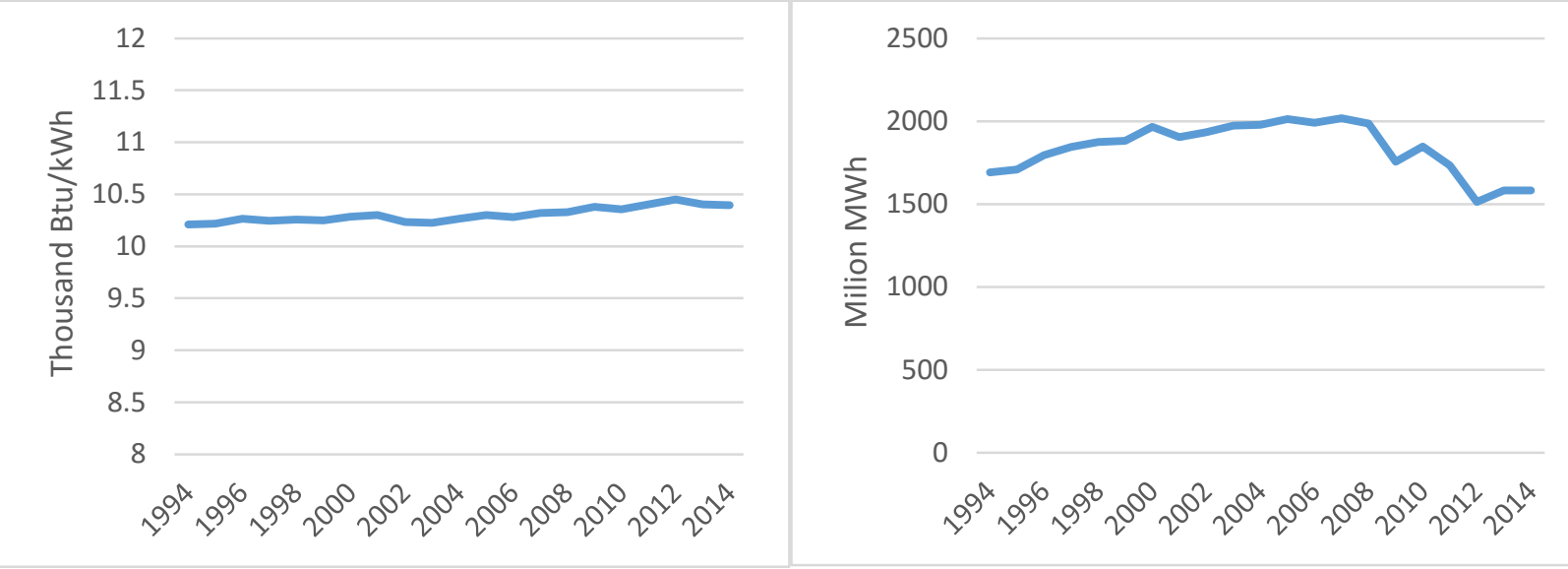

From 1994 to 2014 , heat rates have increased by $1.8 \%$, leading to a slight upward pressure on $\mathrm{SO}_{2}$ emissions. However, electricity generation from coal has declined in recent years after peaking in 2007.

While total electricity generation has increased over the past two decades, the electricity generated by coal plants peaked increased in about 2004, and then declined in the subsequent decade (Figure A.8). The overall reduction in coal generation over the two decades is about $7 \%$ from approximately 1700 million MWh in 1994 to 1580 million MWh in 2004.

During the period from 1994 to total U.S. electricity generation increased while the percent generation from coal declined slightly. From 2004 to 2014, total electricity generation was relatively stable while percent power generation from coal declined from $50 \%$ to $40 \%$ and alternatives like renewables and natural gas became more prevalent, which contributed to the $\mathrm{SO}_{2}$ emission reductions in the most recent decade. 
Figure A.9. Electricity Generation from Coal and Total Generation ${ }^{507}$

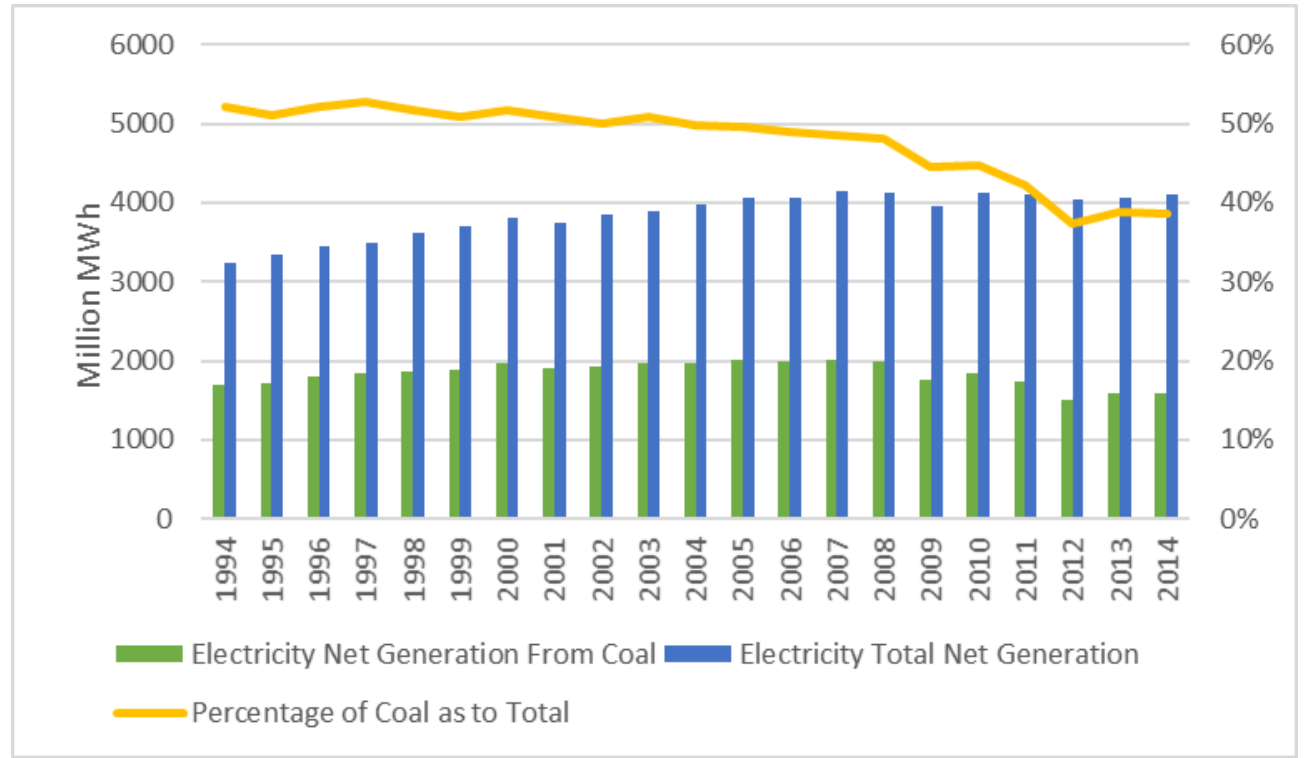

Through 2005, coal accounted for about $50 \%$ of total U.S. electricity generation. In recent years, the amount of electricity generated from coal has declined, contributing to lower $\mathrm{SO}_{2}$ emissions.

\subsection{EMISSION CONTROL: DESULFURIZATION TECHNOLOGY AND REMOVAL}

The final Factor, FGD, suggests a strong and obvious change over the past 20 years. The fraction of emissions lacking flue gas desulfurization (FGD) has declined significantly. FGD is the ratio of emission with flue gas desulfurization to emission without emission control. In other words, it characterizes the proportion of emission left after applying desulfurization technologies. As in Figure A.10, FGD dropped from more than 75\% in 1994 to less than 15\% in 2015. This trend reveals how the application of FGD technology has contributed significantly to the reduction of $\mathrm{SO}_{2}$ emissions. 
Figure A.10. Fraction of Emissions after Flue Gas Desulfurization (FGD) for Coal Plants Figure A.11. Nameplate Net Summer Capacity Percentage with FGD Technology 508

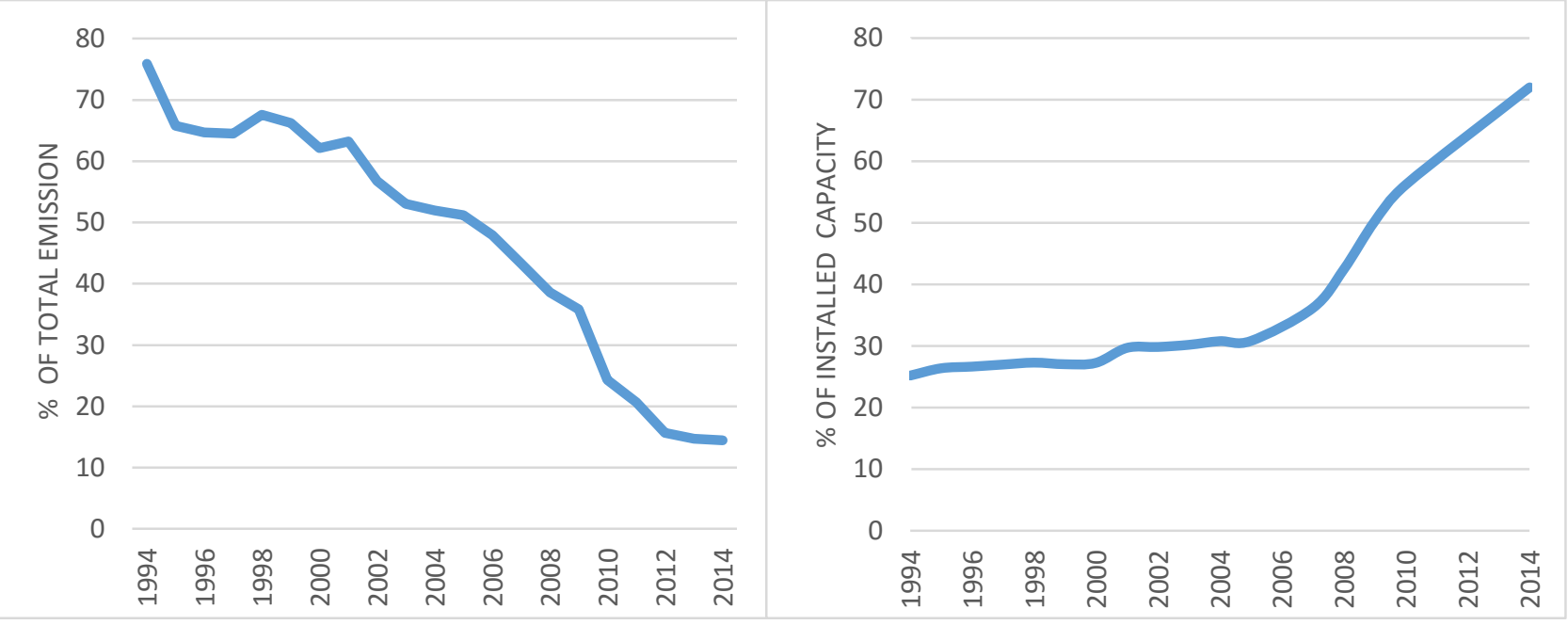

To explain this trend, we decompose the FGD removal rate using two separate contributing: the percent application rate to generation and the removal efficiency of the equipment. Specifically the formula is as follows:

FGD removal Ratio $=\%$ of Application Rate to Generation * Removal Efficiency of Equipment.

The percent FGD application rate to generation is shown in the cumulative market penetration of FGD technology Figure A.11. Following a slow uptake prior to 2005 when approximately $30 \%$ of the nation's summer capacity had FGD technology, FGD technology experienced a more rapid decade of expansion. By 2014, FGD technology accounted was installed on $74 \%$ of the summer capacity of U.S. coal plants. ${ }^{509}$ Unfortunately, we do not have national data on the removal efficiency of FGD equipment used in the power sector. As a result, we assume that it has not changed over the past two decades.

\section{RESULTS: CONTRIBUTION OF EACH FACTOR TO FINAL REDUCTION}

Using the decomposition methodology and measurements described above, we calculate the changes and contributions of different factors that influenced $\mathrm{SO}_{2}$ emission reductions between 1994, when 14.9 million tons of $\mathrm{SO}_{2}$ were emitted, and 2004, when 3.2 million tons of $\mathrm{SO}_{2}$ were emitted. This 11.7 million ton reduction in annual emissions is the subject of the decomposition analysis. The results are summarized in Table A.3 and are portrayed graphically in Figure A.12 and Figure A.13. Figure A.12 displays the decomposition analysis for the entire time period 1994-2004. Figure A.13 shows the dynamics of $\mathrm{SO}_{2}$ emissions individually for two decades: 1994-2004 and 2004-2014. 
Table A.3. 1994-2014 Changes and Contributions of Different Factors

\begin{tabular}{|c|c|c|c|c|c|c|}
\hline & $\begin{array}{l}\text { Emission } \\
\text { (Thousand } \\
\text { Tons) }\end{array}$ & S/Coal (\%) & $\begin{array}{l}\text { E/Coal } \\
\text { (million } \\
\text { Btu/ton) }\end{array}$ & $\begin{array}{l}\text { E/G } \\
\text { (Thousand } \\
\text { Btu/kWh) }\end{array}$ & $\begin{array}{l}\text { G (million } \\
\text { MWh) }\end{array}$ & FGD (\%) \\
\hline 1994 & 14,889 & 1.17 & 20.59 & 10.21 & 1691 & 75.9 \\
\hline 2004 & 10249 & 0.97 & 19.94 & 10.26 & 1978 & 52.0 \\
\hline 2014 & 3,195 & 1.3 & 19.31 & 10.39 & 1582 & 14.4 \\
\hline \multicolumn{7}{|l|}{ 1994-2014 } \\
\hline Difference & $-11,694$ & 0.13 & -1.28 & 0.19 & -109 & -61.5 \\
\hline Change & $-79 \%$ & $11 \%$ & $-6 \%$ & $2 \%$ & $-6 \%$ & $-81 \%$ \\
\hline $\begin{array}{l}\text { Single factor Contributions } \\
\text { (thousand tons } \mathrm{SO}_{2} \text { emission) }\end{array}$ & & 801 & 489 & 137 & -506 & -12614 \\
\hline \multicolumn{7}{|l|}{ 1994-2004 } \\
\hline Difference & $-4,640$ & -0.20 & -0.65 & 0.05 & 287 & -23.9 \\
\hline Change & $-31 \%$ & $-17 \%$ & $-3.2 \%$ & $0.5 \%$ & $17 \%$ & $-32 \%$ \\
\hline $\begin{array}{l}\text { Single factor Contributions } \\
\text { (thousand tons } \mathrm{SO}_{2} \text { emission) }\end{array}$ & & -2329 & 398 & 66 & 1952 & -4701 \\
\hline \multicolumn{7}{|l|}{ 2004-2014 } \\
\hline Difference & $-7,054$ & 0.33 & -0.63 & 0.13 & -396 & -37.6 \\
\hline Change & $-69 \%$ & $34 \%$ & $-3.2 \%$ & $1.3 \%$ & $-20 \%$ & $-72 \%$ \\
\hline $\begin{array}{l}\text { Single factor Contributions } \\
\text { (thousand tons } \mathrm{SO}_{2} \text { emission) }\end{array}$ & & 1772 & 196 & 77 & -1354 & -7757 \\
\hline
\end{tabular}


Figure A.12. Contribution of Each Factor: 1994-2014

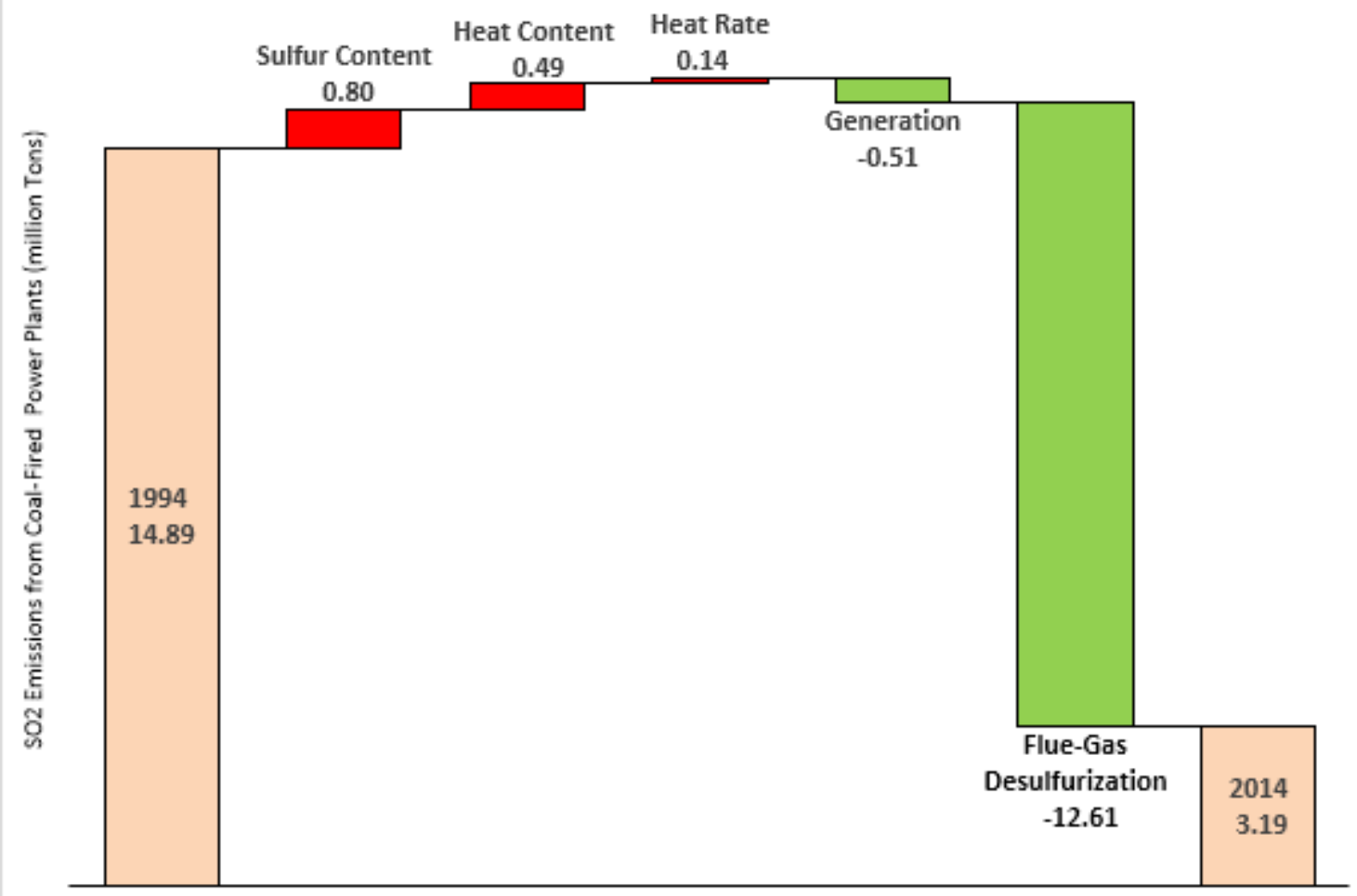

Figure A.13. Contribution of Each Factor: 1994-2004 and 2004-2014

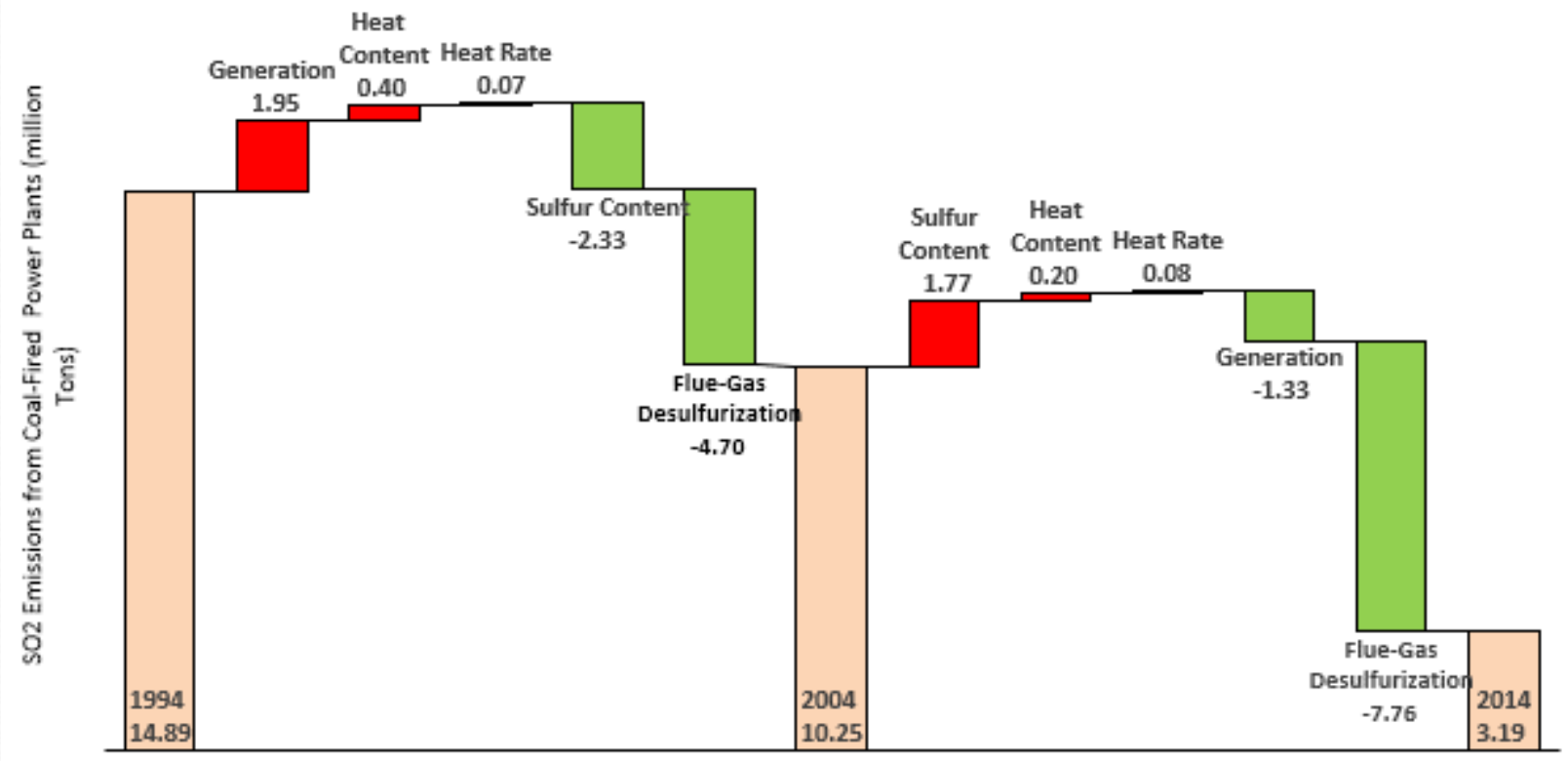


The above figures illustrate that three of the contributing factors have had consistent effects on $\mathrm{SO}_{2}$ emissions over time, while two have had directionally different influences across the two decades.

Flue-gas desulfurization has been responsible for the greatest reduction in $\mathrm{SO}_{2}$ emissions over both decades, accounting for a drop of 4.7 million tons in the first decade and 7.8 million tons in the second decade. The heat content of coal has contributed to small increases in $\mathrm{SO}_{2}$ emissions over both decades, as the overall heat content of coal has decreased. Similarly, the heat rate of coal burning power plants has contributed similarly in both decades to a small uptick in $\mathrm{SO}_{2}$ emissions over both decades, as coal plants have aged and the application of FGD technology comes with an energy penalty.

Between 1994 and 2004, coal generation increased, which would have resulted in an uptick of 2 million tons of $\mathrm{SO}_{2}$ emissions if no other changes had occurred. Over the same decade, however, the sulfur content of coal decreased enough to more than offset the increase in generation. The opposite combination of factors occurred between 2004 and 2014. Coal generation decreased, which would have caused a reduction of 1.3 million tons of $\mathrm{SO}_{2}$ emissions if no other changes had occurred. Over the same decade, however, the sulfur content of coal increased enough to almost offset the decrease in generation.

\section{CONCLUSION}

In conclusion, from 1994 to 2004, the significant $\mathrm{SO}_{2}$ emission reductions from the electricity sector were mainly the result of the massive application of FGD technology. Changing levels of electricity generation from coal plants to alternatives and shifts in the sulfur content of the coal consumed by these plants were the next most influential factors, and their impacts were different and countervailing over the past two decades. The large-scale application of FGD technology since 1994 has hidden these smaller underlying trends, especially the decreasing quality of coal being combusted to produce electricity, both in terms of its increasing sulfur content and its decreasing heat rate. 


\section{APPENDIX B. INTEGRATED ASSESSMENT OF AIR POLLUTION IMPACTS}

\section{Models of Air Pollution Distribution and Atmospheric Interaction}

Oak Ridge National Laboratory and Resources for the Future conducted comprehensive studies of air pollution impacts in the U.S. and the ExternE research program conducted similar research for the European Commission. ${ }^{510} 511$

These studies have emphasized the importance of attributing the observed detrimental effects of air pollution to each individual source of air emissions:

"Throughout the reports in this study, we emphasize that externalities are generally project- and site-specific. The nature of the externalities (e.g., whether they are primarily human health effects or ecological impacts), as well as their size, depend on the design of the power plant, the equipment that is installed, and the location of the power plant." 512

As noted previously, estimating the pollution impact of each power plant requires three components: the spatial distribution of emissions and sources; the ability to track pollution and chemical transformations of pollution in the atmosphere; and the exposure and response of atrisk individuals and assets to pollution. Models that combine all these components in a single coherent framework are called integrated assessment models (IAMs). IAMs are usually complex and costly assessment tools. For this reason the literature relies only on a few of them. IAMs can be used to determine either average or marginal impacts of pollution. Model outputs are sensitive to the assumptions, and data inputs used by the models can result in a range of estimates of air pollution damages.

Three recent studies assess the cost of air pollution from power generation in the U.S.: the EPA 2011 Second Prospective Study of the Clean Air Act; a set of studies developed using the APEEP model; and a review on the externalities of electricity generation by the National Research Council. 513514515516517 A fourth study by the IMF has global scope but also provides insights on air pollution from US power plants. ${ }^{518}$

The Second Prospective Study of the EPA estimates the costs and benefits of the Clean Air Act from 1990 to 2020. The study compares two scenarios: a reference scenario that assumes the 1990 amendments to the Clean Air Act were not passed, and a scenario that assumes passage of the 1990 amendments. ${ }^{519} 520$ The estimated reduction in pollution damages depends on the assumptions used to calculate the impacts of pollution and on the assumptions on pollution trends without the Clean Air Act. 
The National Research Council (NRC) conducted a review of the external cost of energy use in 2010.521 The study covers all fuels, all sectors in which energy is used or transformed and it also covers water pollution and land use impacts of the energy sector. The NRC study relies on the APEEP model for linking emissions of power generation to exposures, for linking exposures to effects and for translating effects into damages that can be monetized. ${ }^{522} 523$ The APEEP model allows attribution of environmental impacts to each power plant and to each pollutant, thus overcoming the limitations of the EPA Prospective Study.

The study assesses impacts on human health, visibility, agriculture, and other sectors of $\mathrm{SO}_{2}$, $\mathrm{NO}_{x}, \mathrm{PM}_{2.5}$ and $\mathrm{PM}_{10}$. The report does not assess the impact of mercury and it has a limited treatment of ecosystem damages. ${ }^{524}$ For example, the study omits from the analysis the eutrophication of fresh-water ecosystems from nitrogen deposition.

The literature consistently finds that the largest negative impacts of air pollution from power generation are on human health. $\mathrm{PM}_{2.5}$ is the pollutant that causes the largest number of deaths, non-fatal heart attacks, and emergency room visits. Most of the effects of exposure to $\mathrm{PM}_{2.5}$ occur during the same year as the exposure. ${ }^{525}$ There is, however, large uncertainty about the exact impacts of air pollution, and estimates of total number of deaths attributable to $\mathrm{PM}_{2.5}$ vary greatly. One of the most frequently used studies is Pope III et al., which is considered to be conservative by a pool of twelve experts surveyed by Roman et al. ${ }^{526} 527$ A more recent study by Schwartz et al. finds three times more deaths from exposure to one microgram of $\mathrm{PM}_{2.5}$ than Pope III et al. ${ }^{528} 529$ Integrated assessment studies of air pollution usually test the robustness of their results using more than one dose-response function from the literature. 


\section{APPENDIX C. VALUATION METHODS OF ENVIRONMENTAL AND HEALTH IMPACTS}

When the market price of the good that is damaged by pollution exists, this price should be used to estimate the marginal benefit of reducing pollution. The aggregate benefit is calculated by multiplying the price by the total number of units of the good that are damaged by pollution. For long-lived goods (such as timber forests) the impact should be discounted. Examples of market externalities of power generation include crop and timber productivity losses and deterioration of materials. Crop and timber market prices can be used to estimate the value of the production loss. The additional discounted maintenance cost or the replacement cost of surfaces over the lifetime of structures due to pollution can be used to estimate material deterioration costs.

Unfortunately, the largest externalities from power generation impact goods that are not traded in markets. This means that there is not a price that can be attached to those goods. Examples of non-market externalities of power generation include health impacts, ecosystem impacts, and amenity value losses due to deterioration of air quality.

There are two broad classes of methods to estimate the value of non-market goods. The first class of methods infers the value that consumers give to non-market goods by observing their consumption choices. These are called revealed preference methods. The second class of methods relies on a direct consultation with consumers. Researchers directly ask consumers what is the monetary value that they attach to some goods that are not market traded. These are called stated preferences methods. The literature has used both classes of methods to estimate the market value of a wide range of non-market goods.

\section{Human Life and Health}

Increased morbidity and mortality are responsible for the largest share of external costs of power generation. 530531532533534 The largest differences between estimates of the external cost of power generation are due to differences in the economic value of health and human life losses. It is therefore useful to understand the method used to estimate the economic impact of pollution on human health.

The economic cost of increased morbidity is typically estimated by using the cost of health care. For example, increased asthma attacks require increased use of medications, which have a market price. Exposure to ozone may lead to emergency room visits, the cost of which can be estimated. Long-term health care costs due to impairing conditions can also be estimated and discounted to attach a cost to present-day emissions. 
Estimating the economic value of a human life is relatively more difficult. Economists have developed methods for applying economic valuation to human life based on the following consideration. This starts with the recognition that individuals commonly make risky choices that increase, either directly or indirectly, their probability of dying. For example, workers show a preference for safe jobs but some of them are willing to take more risks than others. By aggregating all the risk-wage combinations observed across many workers and across industries, researchers estimate the aggregate relationship between risk and wages. Many studies have shown that this relationship is positive: workers require higher wages to accept more risk. ${ }^{535}$ This is an intuitive result that implies the non-intuitive conclusion that workers are willing to trade off their health and life in general for higher economic compensation. For example, the wage-risk equation may reveal that workers require a wage increase equal to $\$ 600$ to increase the risk of death from .0001 to .0002. This means that in the aggregate there will be one extra death every ten thousand workers. The aggregate compensation to accept this additional death is equal to $\$ 6$ million. ${ }^{\text {hhh }}$ For the employer - or for society at large - the value of reducing the probability of mortality by $1 / 1000$ is then equal to $\$ 6$ million. This metric is called the value of a statistical life (VSL): the amount of money that individuals are willing to pay to reduce by a small amount the probability of death. The VSL is used by economist to monetize human life and is an estimate of how much society is willing to pay to reduce the probability of death by an arbitrarily small amount.

One critique of the VSL is that it does not distinguish between young and old people. Economists correct this problem by using a modified version of the VSL that accounts for the years of expected life lost. This is called the value of the statistical life-years (VSLY).

Viscusi and Aldy find that the value of the VSL ranges between $\$ 6-9$ million in most U.S. studies. As income increases, the compensation required to increase risk increases and so does the VSL. ${ }^{536}$ Estimates show that as income increases by $10 \%$, the VSL increases by $5-6 \%$. This implies that the socially optimal value of pollution will decline as income increases.

The EPA uses $\$ 620$ for a risk reduction equal to $1 / 10,000$. This implies a VSL equal to $\$ 6.2$ million.

\section{Ecosystems}

Impacts on ecosystems are hard to quantify and are not always as accurate as for other sectors. ${ }^{537}$ Ecosystems provide many services to the economy, most of which are not monetized. However, this does not mean that the environmental goods have no economic value.

hhh This example is drawn from Kolstad (2010). 
Environmental economists have developed several methods to infer the value of environmental goods that are not traded in markets. The goal of these methods is to estimate the willingness to pay of consumers to enjoy an environmental good - the use value - or to protect its existence - the existence value.

There are two broad classes of methods to estimate the willingness to pay. The first group relies on revealed preferences. For the VSL, researchers estimate the value of the non-market good by observing actual consumers' choices. Some studies estimate the value of the environmental good (view, temperature, quiet...) by empirically determining how much consumers spend to enjoy an extra unit of the environmental good. For example, consumers are usually willing to pay more for houses that have a better view or that are on a less polluted street. By comparing two possibly identical houses in all respects but view or pollution level, the economist can attribute an economic value to the two environmental characteristics. These are called hedonic methods.

Other methods determine the value of harmful environmental conditions (e.g. pollution) or the value of natural beauties, cultural and historic sites by estimating how much consumers spend on defensive measures (from pollution) or on measures that allow them to fully enjoy the environmental amenity. These are called household production methods. Defensive expenditures (e.g. pollution masks or air filters) provide an indication of how much consumers dislike the environmental bad. Travel costs are an example of expenditures that allow consumers to fully enjoy natural beauty, quiet and other environmental amenities.

The second class of methods relies on constructed markets to estimate consumers' willingness to pay for environmental goods. These methods rely on questioning consumers about their willingness to pay to enjoy or protect an environmental good. Researchers use stated preferences rather than revealed preferences. This method is known as contingent evaluation because consumers are asked questions about their preferences assuming the existence of a market. Contingent valuation methods are controversial because of their hypothetical nature. Contingent valuation studies must be carefully designed to provide reliable estimates and their use is usually limited to estimating the existence value of natural goods. For example, several contingent valuation studies have assessed how much consumers are willing to pay to preserve wilderness in places that they have never and will likely never visit in the future, such as the Arctic 


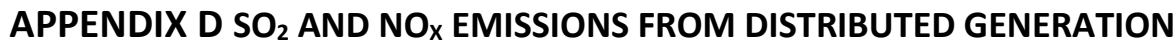

The U.S. has more than 12 million distributed generation units, ${ }^{538}$ including distributed wind, distributed solar photovoltaics (i.e. "rooftop solar"), and combined heat-and-power (CHP) plants. Distributed generation technologies that involve combustion-particularly burning fossil fuels - can produce many of the same types of emission as larger fossil-fuel-fired power plants, including air pollution and greenhouse gases. Because emissions from distributed generation are typically not included in electric power sector totals, iii they must be accounted for separately. Figure D.1 shows recent trends in distributed electricity generation by fuel type.

Figure D.1. Distributed Generation by Fuel, 2001-2015.539 540

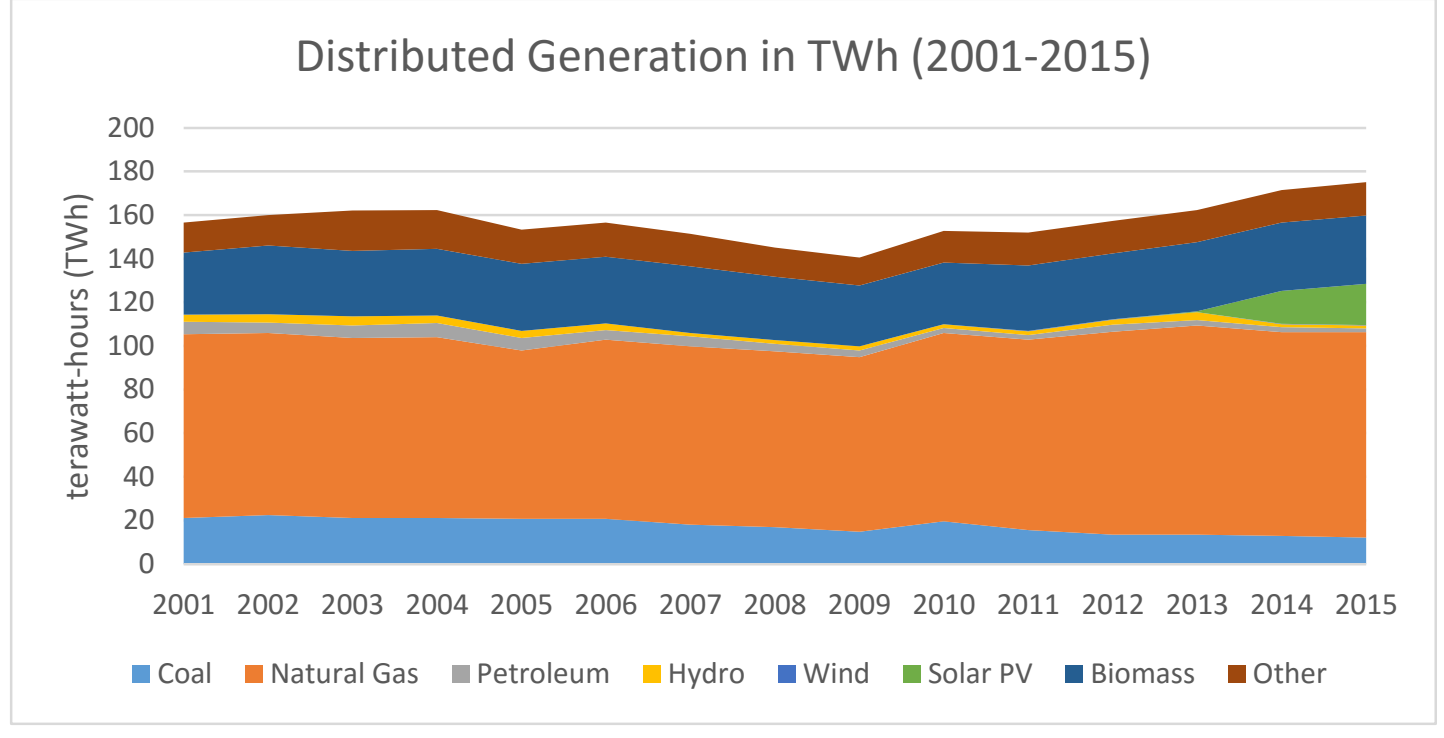

Notes: Solar includes utility-scale solar and distributed solar. The EIA began tracking distributed solar in 2014. This lack of data for prior years explains the apparent jump in solar generation from 2013 to 2014. Other includes nonbiogenic municipal solid waste, batteries, hydrogen, purchased steam, sulfur, tire-derived fuel, and other miscellaneous energy sources.

In 2013-the latest year for which emissions data is available-total distributed generation included 12.52 terawatthours (TWh) of electricity from the commercial sector and 150.02 TWh

\footnotetext{
iii The electric power sector is defined in the North American Industry Classification System (NAICS) as the energyconsuming sector that consists of electricity-only and combined-heat-and-power (CHP) plants whose primary business is to sell electricity, or electricity and heat, to the public-i.e., NAICS 22 plants. Distributed generation refers to electricity or combined heat-and-power that is consumed on-site, without being sold through the bulk power system. In national inventories such as the National Emissions Inventory, emissions from distributed generation are typically assigned to the end-use sector-industrial, commercial or residential-in which the generation and consumption takes place.
} 
from the industrial sector. Total 2013 emissions resulting from distributed generation included 616,697 metric tons of $\mathrm{SO}_{2}$ and 354,263 metric tons of $\mathrm{NO}_{x}$ (Figure D.2).

The generation mix and emissions profile of distributed generation differs in several important ways from electricity generation in the power sector. For example, in 2015, 96 percent of distributed generation (excluding small-scale solar) was generated at combined heat-andpower (CHP) plants, with only 4 percent of distributed electricity generated at non-CHP facilities. In the power sector, these numbers are reversed-96\% of electricity is generated at electricity-only utilities and independent power producers, and only 4 percent of electricity is generated at CHP plants in the power sector. ${ }^{541}$

CHP generates electricity and useful hot water or steam from a single system at or near the point of use. In general, CHP systems use 25-35 percent less primary energy than using grid electricity plus conventional heating end-uses (e.g., water heaters or boilers) and typically have greater efficiencies than heaters using grid electricity. Note that, because CHP systems generate both useful thermal energy and electricity, an output-based emission rate-amount of $\mathrm{SO}_{2}$ or $\mathrm{NO}_{\mathrm{x}}$ emitted per productive outputs (Ib emission/useful energy) - is a better measure of the environmental impact than an emission rate based on the amount of fuel burned. An output-based rate helps recognize the pollution prevention benefits of efficient generation technologies such as CHP. More information about distributed generation is included in the End Use baseline.

The generation mix and subsequent emissions of distributed generation differs significantly from the power sector generation mix. Natural gas comprised $59 \%$ of distributed generation in 2013 , while coal accounted for $8.3 \%$ of distributed generation. Renewables also make up a relatively larger portion of distributed generation, with biomass, hydropower, and other renewables accounting for $19 \%, 2 \%$ and $0.3 \%$ of electricity generated at distributed facilities in $2013 . .^{542 \mathrm{jj}}$

$\mathrm{jjj}$ Totals include distributed generation at utility-scale facilities (> $1 \mathrm{MW}$ capacity). EIA began tracking generation from small-scale solar (<1 MW capacity) in 2014. 
Figure D.2. $\mathrm{SO}_{2}$ and $\mathrm{NO}_{x}$ Emissions from Distributed Generation, 2001-2013. ${ }^{543}$

\section{$\mathrm{SO}_{2}$ and $\mathrm{NO}_{x}$ Emissions from Distributed Generation}

(2001-2013)

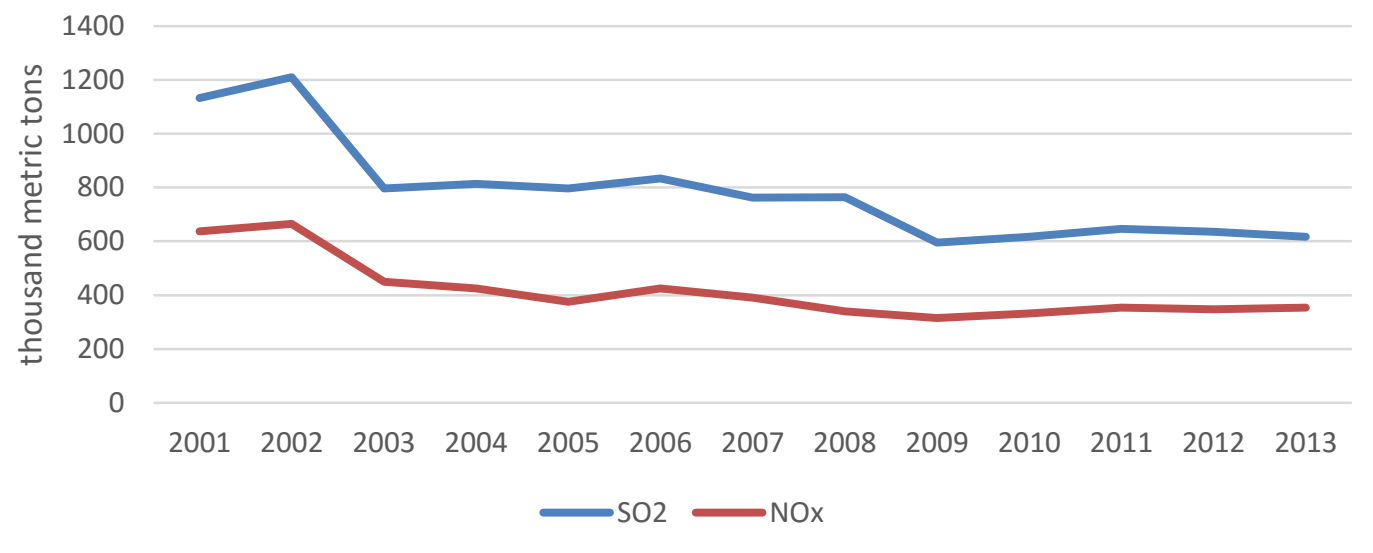




\section{APPENDIX E LIFE CYCLE IMPACTS OF ELECTRICITY GENERATION}

The air quality and land-use impacts addressed elsewhere in this baseline include only the direct impacts from electricity generation. Additional emissions, land-use impacts, and other environmental impacts are associated with other stages in the full life cycle of an electricity generation technology. In particular, "upstream" impacts include impacts from raw material extraction, component manufacturing, and facility construction. Similarly, "downstream" impacts include impacts from waste management and one-time impacts from the decommissioning, disassembly, and disposal or recycling of equipment and other facility materials. Additional ongoing non-generation-related emissions result from power plant operation and maintenance activities.

Figure E.1. Generalized Life-Cycle Stages for Energy Technologies. ${ }^{544}$

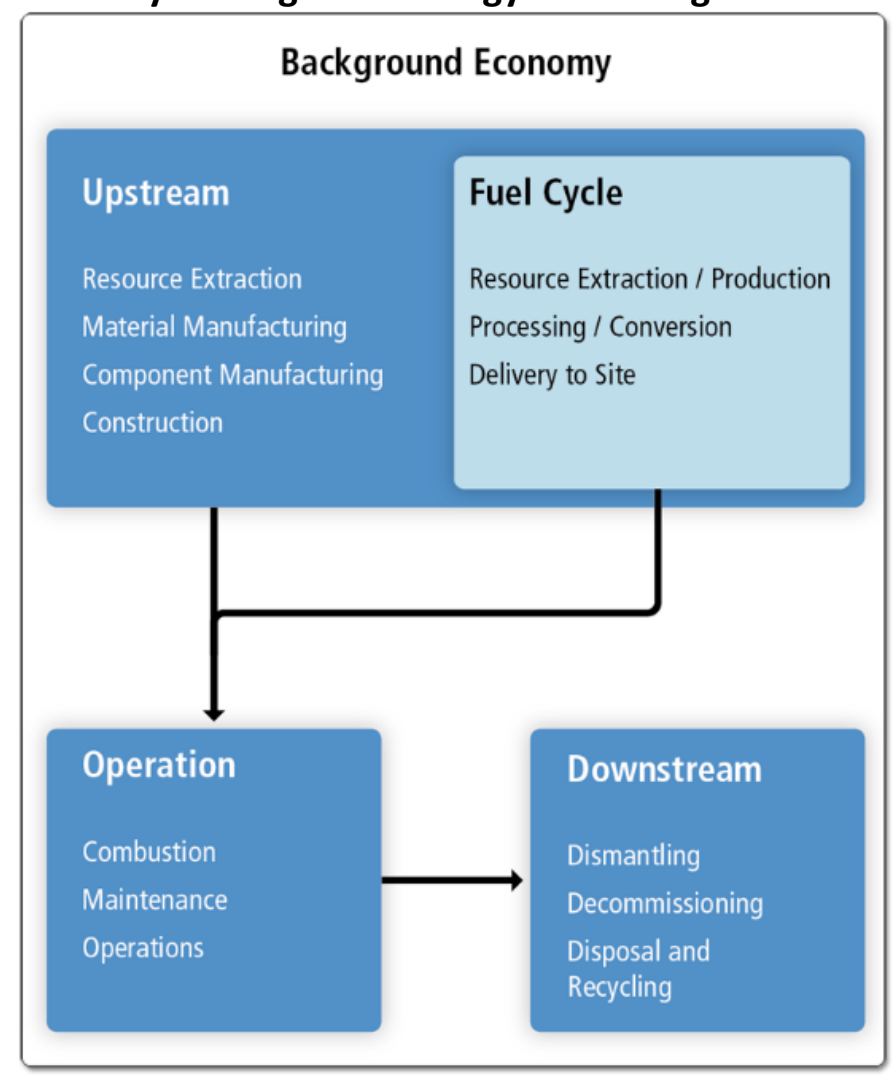

Life cycle assessments (LCAs) provide a holistic or system-level assessment of the impacts of electricity generation-including direct and indirect greenhouse gas (GHG) emissions, air quality, land-use, water withdrawal and consumption, and waste streams. Ideally, an LCA will characterize all flows of energy, resources, and pollutants across the life cycle of a technology and its supporting systems, including activities related to raw materials acquisition, 
manufacturing, transportation, installation and maintenance, operation, and decommissioning. ${ }^{545}$

Life cycle impacts are often measured as an emission/impact factor, or a ratio of the amount of impact per the amount of electricity generated. For example, life cycle $\mathrm{PM}_{2.5}$ emissions are often measured as a rate in terms of grams of $\mathrm{PM}_{2.5}$ emitted per megawatt-hour of electricity generated ( $\mathrm{g} \mathrm{PM}_{2.5} / \mathrm{MWh}$ ). The life cycle $\mathrm{PM}_{2.5}$ emission rate for a particular fuel would include the direct emissions from generation (see Section 2), as well as any additional emissions associated with electricity generation, such as mining or other fuel procurement activities.

An emission or impact factor is suitable for impacts of regular operation, for example, fuel cycle impacts. Impacts which are associated with one-time upstream or downstream activities may be more appropriately measured as a function of the size of the generating plant and are often measured in terms of impact per electric generating capacity (e.g., land use per megawatt of generating capacity). One-time impacts (per capacity) may be converted to impact rates (per electricity generation) by using assumptions about the total operating life and total energy output of a power plant.

Substantial variability in published LCAs result from technology characteristics (e.g., design, capacity factor, variability, service lifetime and vintage), geographic location, background energy system characteristics, data source, and differences in LCA methodology and assumptions. ${ }^{546}$ LCA guidelines ${ }^{547}$ and the International Reference Life Cycle Data System $H_{a n d b o o k^{548}}$ have been developed to provide coherence and comparability among LCA studies. However, harmonization of LCA methodology and assumptions remains a difficult task. Consequently, this appendix includes ranges for all environmental impacts based on a literature review of LCA studies.

\section{Air Quality and Other Life Cycle Impacts from Electricity Generation}

According to the World Health Organization, the air emissions associated with electricity generation that have the most significant impacts on human health include sulfur dioxide $\left(\mathrm{SO}_{2}\right)$, nitrogen oxides $\left(\mathrm{NO}_{\mathrm{x}}\right)$, particulate matter $\left(\mathrm{PM}_{2.5}\right)$, and non-methane volatile organic compounds (NMVOC). ${ }^{549}$ In 2011, the Intergovernmental Panel on Climate Change conducted a literature review of published LCAs to assess life cycle emissions of these pollutants across a wide range of fuel and technology types and includes global and regional studies. ${ }^{550}$ Figure E.2displays the results of this review.

For each pollutant in Figure E.2, the quantity of pollutant emitted per unit of electricity is displayed as a range, with the light-colored bar indicating the minimum estimated life cycle emissions, and the dark-colored bar displaying the maximum estimate for life cycle emissions. Several factors contribute to the wide range of emission factors. In particular, the thermal 
efficiency of electricity generation, the availability of pollution control technology, and regional variations in fuel quality all contribute to the considerable variation in estimates for life cycle emissions. The results for fossil electricity generation include country-specific averages for current technology and fuel supply for the United States, China, all European, and a few other countries. Minimum and maximum values mainly represent the countries with the most and least efficient power plant and pollution control technology, respectively.

This assessment finds that non-combustion renewable energy technologies and nuclear power result in comparatively minor emissions of air pollutants, solely from upstream and downstream processes. Also the range of emissions across country and LCA study is in general much lower for renewable energy and nuclear than for fossil power. For fossil power, emissions at the higher end of the ranges shown typically apply to developing economies that use older technologies, have less pollution control measures in place, may consume lowerquality fuels, and may have fewer environmental regulations.

Other life cycle impacts from electricity generation include impacts to water quality, land use, greenhouse gas emissions, and other environmental factors. Note that land use life cycle impacts are discussed in more detail in Chapter 3. Life cycle water use and greenhouse gas emissions are discussed in other baseline reports. 
Figure E.2. Life Cycle Emissions of Air Pollutants from Electricity Generation. ${ }^{551}$
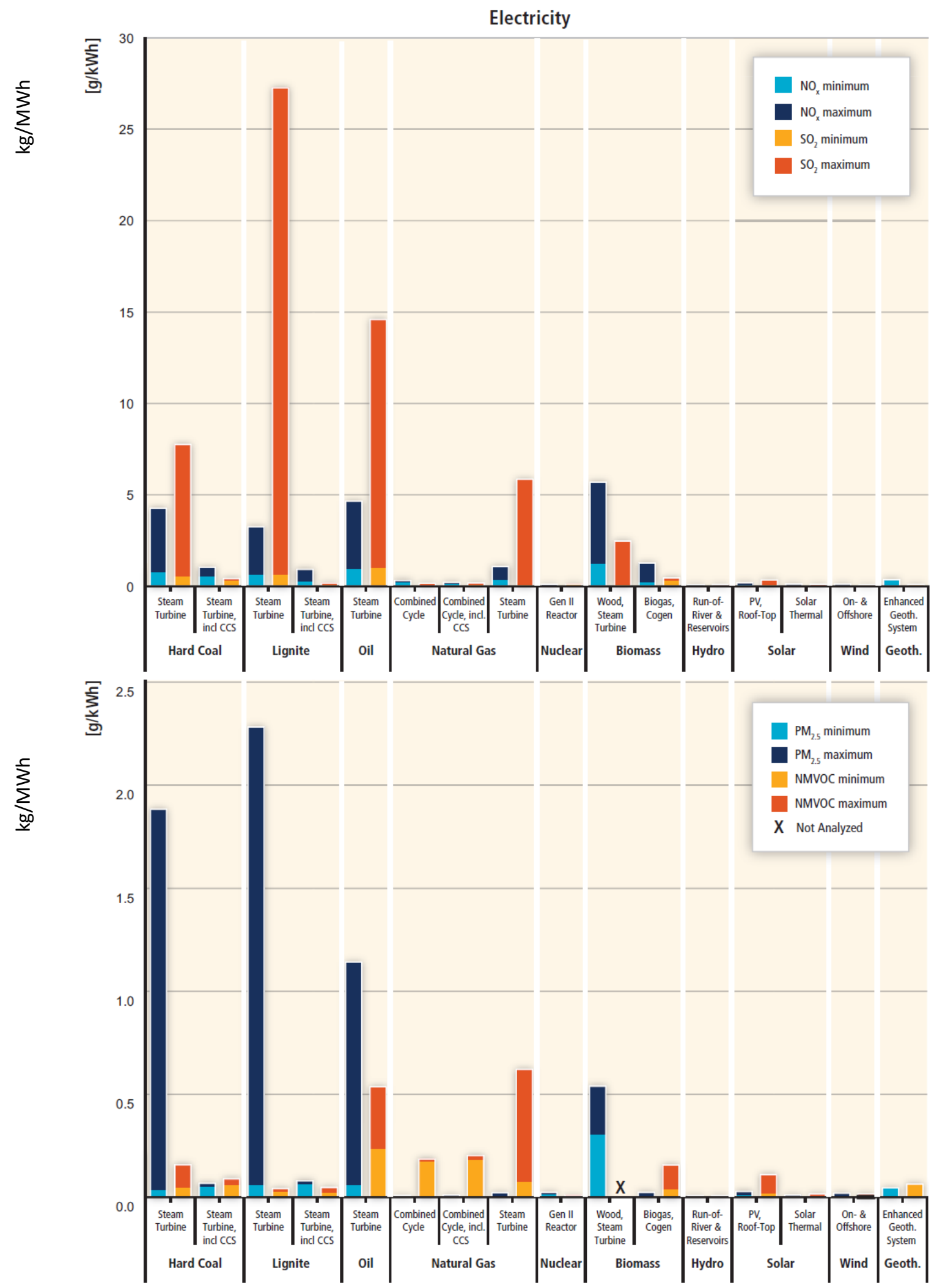

Note: Emission rates are displayed in kilograms of pollutant per megawatt-hour of electricity. 
${ }^{1}$ Projections analysis conducted by the DOE Office of Energy Policy and Systems Analysis (EPSA), February 2016.

${ }^{2} \mathrm{SO}_{2}$ and $\mathrm{NO}_{x}$ historical emissions from EPA. "Air Pollutant Emissions Trends Data." https://www.epa.gov/sites/production/files/2015-07/national tier1 caps.xlsx. Accessed June 1, 2016.

${ }^{3}$ Mercury historical emissions 1990-2005 from EPA. "Report on the Environment: Mercury Emissions." https://cfpub.epa.gov/roe/download/051 mercury-emissions exh-1.csv. Accessed June 1, 2016. Mercury historical emissions in 2008 and 2011 from EPA. "National Emissions Inventory 2008 and 2011." https://www.epa.gov/air-emissions-inventories/2011-national-emissions-inventory-nei-data and https://www.epa.gov/air-emissions-inventories/2008-national-emissions-inventory-nei-data. Accessed June 1, 2016.

${ }^{4}$ Department of Energy. “Quadrennial Energy Review: Energy Transmission, Storage and Distribution Infrastructure." p 7-3 - 7-6. April 2016. http://www.energy.gov/epsa/quadrennial-energy-review-first-installment ${ }^{5}$ White House, 2015. "Modernizing our Electric transmission Infrastructure and Driving the Development of Clean Energy." July, 2015 Report. https://www.whitehouse.gov/blog/2015/07/21/modernizing-our-electrictransmission-infrastructure-and-driving-development-clean-e ${ }^{6}$ Brown, Marilyn A., Daniel D'Arcy, Melissa Lapsa, Isha Sharma, Yufei Li. Solid Waste from the Operation and Decommissioning of Power Plants, Oak Ridge National Laboratory, ORNL/SPR-2016/774 (2016).

${ }^{7}$ Cunliff C. and C. Murphy, Environment Baseline Volume 1: Greenhouse Gas Emissions from the U.S. Power Sector, Office of Energy Policy and Systems Analysts, U.S. Department of Energy, May 2016.

7 EPA. "Inventory of U.S. Greenhouse Gas Emissions and Sinks: 1990-2013." p.1-3. April 2015.

${ }^{8}$ Energy Information Administration. Electric Power Annual. Tables 4.1 and 3.2-3.3. February 2016. http://www.eia.gov/electricity/annual/. Accessed April 27, 2016.

${ }^{9}$ Energy Information Administration (EIA). Electricity Data Browser. 2015. http://www.eia.gov/electricity/data/browser/. Accessed March 17, 2016.

${ }^{10}$ Marcy, C., J. Logan, J. McCall, F. Flores-Espino, A. Bloom, J. Aabakken, W. Cole, T. Jenkins, and G. Porro,

"Electricity Generation Baseline Report," National Renewable Energy Lab (2016).

${ }^{11}$ Energy Information Administration (EIA). Electricity Data Browser. 2015. http://www.eia.gov/electricity/data/browser/. Accessed March 17, 2016.

${ }^{12}$ Environmental Protection Agency. 2011 National Emissions Inventory, version 2. Technical Support Document. https://www.epa.gov/sites/production/files/2015-10/documents/nei2011v2 tsd_14aug2015.pdf Accessed April 11, 2016. Table 2-2, p. 24.

${ }^{13}$ Cunliff C. and C. Murphy. "Environment Baseline Volume 1: Greenhouse Gas Emissions from the U.S. Power Sector." Office of Energy Policy and Systems Analysts, U.S. Department of Energy, May 2016.

${ }^{14}$ Industrial Economics. Health and Welfare Benefits Analyses to Support the Second Section 812 Benefit-Cost Analysis of the Clean Air Act. (Cambridge, MA: Industrial Economics, 2011).

${ }^{15}$ Environmental Protection Agency. 2008. 2008 Final Report: Integrated Science Assessment (ISA) for Sulfur Oxides - Health Criteria. U.S. Environmental Protection Agency, Washington, DC, EPA/600/R-08/047F, 2008.

Chapter 3, p. 3-1. http://ofmpub.epa.gov/eims/eimscomm.getfile?p download id=491274.

${ }^{16}$ Environmental Protection Agency. 2011. The Benefits and Costs of the Clean Air Act from 1990 to 2020. U.S. Environmental Protection Agency, Office of Air and Radiation, Final Report, Rev A. Exhibit 8, p. 14.

https://www.epa.gov/sites/production/files/2015-07/documents/fullreport rev a.pdf.

${ }^{17}$ Environmental Protection Agency. 2011. National Acid Precipitation Assessment Program Report to Congress 2011: An Integrated Assessment. P. ES1. http://ny.water.usgs.gov/projects/NAPAP/NAPAP 2011 Report 508 Compliant.pdf.

${ }^{18}$ Environmental Protection Agency. 2008. Integrated Science Assessment for Oxides of Nitrogen - Health Criteria United States Environmental Protection Agency, EPA/600/R-08/071. Figure 2.21, p. 2-3.

http://ofmpub.epa.gov/eims/eimscomm.getfile?p download id=475020.

${ }^{19}$ Environmental Protection Agency. 2009. Integrated Science Assessment for Particulate Matter National Center for Environmental Assessment-RTP Division Office of Research and Development U.S. Environmental Protection 
Agency Research Triangle Park, NC. EPA/600/R-08/139F. P. 2-5 - 2-8.

http://ofmpub.epa.gov/eims/eimscomm.getfile?p download id=523389.

${ }^{20}$ Environmental Protection Agency. 2009. Integrated Science Assessment for Particulate Matter National Center for Environmental Assessment-RTP Division Office of Research and Development U.S. Environmental Protection Agency Research Triangle Park, NC. EPA/600/R-08/139F. P. 2-5.

http://ofmpub.epa.gov/eims/eimscomm.getfile?p download id=523389.

${ }^{21}$ Environmental Protection Agency. 2010. Integrated Science Assessment for Carbon Monoxide. National Center for Environmental Assessment-RTP Division, Office of Research and Development, U.S. Environmental Protection Agency, Research Triangle Park, NC, EPA/600/R-09/019F. P. 2-4.

${ }^{22}$ Environmental Protection Agency. Ozone Basics. https://www.epa.gov/ozone-pollution/ozone-basics\#effects, Accessed April 11, 2016.

${ }^{23}$ Environmental Protection Agency. Ozone Basics. https://www.epa.gov/ozone-pollution/ozone-basics\#effects, Accessed April 11, 2016.

${ }^{24}$ Environmental Protection Agency. Hazardous Air Pollutants. https://www.epa.gov/haps. Accessed April 11, 2016.

${ }^{25}$ ElA. "Monthly Energy Review." Table 7.2b. http://www.eia.gov/totalenergy/data/monthly/. Accessed April 19, 2016.

${ }^{26}$ EPA. "National Emissions Inventory." https://www.epa.gov/air-emissions-inventories/national-emissionsinventory. Based on data for the "Fuel Comb - Electric Generation - fuel" sectors. Accessed April 19, 2016.

${ }^{27}$ ElA. "Monthly Energy Review." Table 7.2b. http://www.eia.gov/totalenergy/data/monthly/. Accessed April 19, 2016.

${ }^{28}$ EPA. "National Emissions Inventory." https://www.epa.gov/air-emissions-inventories/national-emissionsinventory. Based on data for the "Fuel Comb - Electric Generation - fuel" sectors. Accessed April 19, 2016.

${ }^{29}$ Environmental Protection Agency. Air Pollutant Emissions Trends Data, 1970-2014: https://www.epa.gov/airemissions-inventories/air-pollutant-emissions-trends-data. Accessed April 7, 2016.

${ }^{30}$ Energy Information Administration. Monthly Energy Review. 2016: Table 7.2b Electricity Net Generation: Electric Power Sector: http://www.eia.gov/totalenergy/data/monthly/. Accessed April 7, 2016.

${ }^{31}$ Environmental Protection Agency. Air Pollutant Emissions Trends Data, 1970-2014: https://www.epa.gov/airemissions-inventories/air-pollutant-emissions-trends-data. Accessed April 7, 2016.

32 Energy Information Administration. Monthly Energy Review. 2016: Table 7.2b Electricity Net Generation: Electric Power Sector: http://www.eia.gov/totalenergy/data/monthly/. Accessed April 7, 2016.

${ }^{33}$ Environmental Protection Agency. Air Pollutant Emissions Trends Data, 1970-2014: https://www.epa.gov/airemissions-inventories/air-pollutant-emissions-trends-data. Accessed April 7, 2016.

${ }^{34}$ Energy Information Administration. Monthly Energy Review. 2016: Table 7.2b Electricity Net Generation: Electric Power Sector: http://www.eia.gov/totalenergy/data/monthly/. Accessed April 7, 2016.

${ }^{35}$ Environmental Protection Agency. Air Pollutant Emissions Trends Data, 1970-2014.

${ }^{36}$ Energy Information Administration. Monthly Energy Review. 2016: Table 7.2b Electricity Net Generation: Electric Power Sector.

${ }^{37}$ Environmental Protection Agency. Air Pollutant Emissions Trends Data, 1970-2014. https://www.epa.gov/airemissions-inventories/air-pollutant-emissions-trends-data. Accessed April 7, 2016.

${ }^{38}$ Environmental Protection Agency. Air Pollutant Emissions Trends Data, 1970-2014. https://www.epa.gov/airemissions-inventories/air-pollutant-emissions-trends-data. Accessed April 7, 2016.

${ }^{39}$ Environmental Protection Agency. Air Pollutant Emissions Trends Data, 1970-2014. https://www.epa.gov/airemissions-inventories/air-pollutant-emissions-trends-data. Accessed April 7, 2016.

${ }^{40}$ Environmental Protection Agency. untitled manuscript: https://www3.epa.gov/ttnchie1/ap42/ch01/final/c01s04.pdf, p.2.

${ }^{41}$ Environmental Protection Agency. https://www3.epa.gov/airquality/carbonmonoxide/.

${ }^{42}$ Environmental Protection Agency. 2010. Integrated Science Assessment for Carbon Monoxide. National Center for Environmental Assessment-RTP Division, Office of Research and Development, U.S. Environmental Protection Agency, Research Triangle Park, NC, EPA/600/R-09/019F. P. 2-4.

${ }^{43}$ Environmental Protection Agency. Air Pollutant Emissions Trends Data, 1970-2014. 
${ }^{44}$ EIA. “Detailed State Data." U.S. Electric Power Industry Estimated Emissions by State. October 2015. http://www.eia.gov/electricity/data/state/. Power sector emissions include emissions from electric utilities and independent power producers. Accessed March 2, 2016.

${ }^{45}$ Environmental Protection Agency. https://www3.epa.gov/mats/powerplants.html.

${ }^{46}$ Environmental Protection Agency. 2014. Data from the 2011 National Emissions Inventory, Version 1. http://www.epa.gov/ttn/chief/net/2011inventory.html.

${ }^{47}$ EIA (2015). "What is the efficiency of different types of power plants?" http://www.eia.gov/tools/faqs/faq.cfm?id=107\&t=3. Accessed January 19, 2016.

${ }^{48}$ EIA. "Analysis of Heat Rate Improvement Potential at Coal-Fired Power Plants." May 2015. http://www.eia.gov/analysis/studies/powerplants/heatrate/. p. 1. Accessed January 21, 2016.

${ }^{49}$ EIA. "Annual Electric Generator Report." https://www.eia.gov/electricity/data/eia860. Accessed May 10, 2016.

${ }^{50}$ ElA. "Monthly Energy Review." http://www.eia.gov/totalenergy/data/monthly. Accessed May 10, 2016.

${ }^{51}$ EIA. "Form EIA-923 Detailed Data." https://www.eia.gov/electricity/data/eia923. Accessed May 10, 2016.

52 EPA. "National Emissions Inventory." http://www.epa.gov/air-emissions-inventories/2011-national-emissionsinventory-nei-data. Accessed May 10, 2016.

${ }^{53}$ Environmental Protection Agency. "National Emissions Inventory." 2011. https://www.epa.gov/air-emissionsinventories/2011-national-emissions-inventory-nei-data. Accessed March 17, 2016.

${ }^{54}$ EPA. "Technical Support Document: Estimating the Benefit per Ton of Reducing PM2.5 Precursors from 17

Sectors." p. 101. January 2013. https://www.epa.gov/sites/production/files/2014-

10/documents/sourceapportionmentbpttsd.pdf. Accessed June 1, 2016.

${ }^{55}$ Environmental Protection Agency. https://www.epa.gov/international-cooperation/mercury-emissions-globalcontext.

${ }^{56}$ Travnikov, Oleg. "Contribution of the intercontinental atmospheric transport to mercury pollution in the Northern Hemisphere." Atmospheric Environment 39, no. 39 (2005): 7546.

${ }^{57}$ Drevnicxk et al. "Spatial and temporal patterns of mercury accumulation in lacustrine sediments across the Laurentian Great Lakes region." Environmental Pollution, 161 (2012), 252-260. Page 258.

http://www.sciencedirect.com/science/article/pii/S0269749111003125.

58 UNEP, 2013. "Global Mercury Assessment 2013: Sources, Emissions, Releases

and Environmental Transport." p 23 UNEP Chemicals Branch, Geneva, Switzerland.

http://www.unep.org/PDF/PressReleases/GlobalMercuryAssessment2013.pdf. Accessed June 2, 2016.

${ }^{59}$ Travnikov, Oleg. "Contribution of the intercontinental atmospheric transport to mercury pollution in the Northern Hemisphere." 2005: 7546.

${ }^{60}$ National Atmospheric Deposition Program (NRSP-3). 2015. NADP Program Office, Illinois State Water Survey, University of Illinois, Champaign, IL 61820.

${ }^{61}$ EPA. "Regulatory Impact Analysis for the Final Mercury and Air Toxics Standards." p 7-35 through 7-39. EPA452/R-11-011. December 2011. https://www3.epa.gov/ttn/ecas/regdata/RIAs/matsriafinal.pdf. Accessed June 2, 2016.

${ }^{62}$ Castro M.S. \& J. Sherwell, Effectiveness of emission controls to reduce the atmospheric concentrations of mercury, Envtl. Sci. Tech., 49(24): 14000-14007 (2015).

${ }^{63}$ Drevnick P.E., et al., Spatial and temporal patterns of mercury accumulation in lacustrine sediments across the Great Lakes region. Environmental Pollution. 161: 252-260 (2012).

${ }^{64}$ Evers D.C. et al., Biological mercury hotspots in the northeastern United States and southeastern Canada, Bioscience, 57(1): 29-43 (2007).

${ }^{65}$ Giang A. \& N.E. Selin, Benefits of mercury controls for the United States, PNAS (Early Edition) (2015), http://www.pnas.org/cgi/doi/10.1073/pnas.1514395113.

${ }^{66}$ Hutcheson, M.S. et al., Temporal and spatial trends in freshwater fish tissue mercury concentrations associated with mercury emissions reductions, Envtl. Sci. Tech., 48: 2193-2202 (2014).

${ }^{67}$ UNEP, 2013. "Global Mercury Assessment 2013: Sources, Emissions, Releases

and Environmental Transport." p 23 UNEP Chemicals Branch, Geneva, Switzerland. http://www.unep.org/PDF/PressReleases/GlobalMercuryAssessment2013.pdf. Accessed June 2, 2016.

${ }^{68}$ EPA website. "Cap and Trade 101." https://www3.epa.gov/captrade/captrade-101.html. Accessed May 4, 2016. 
${ }^{69}$ NAPAP. National Acid Precipitation Assessment Program Report to Congress: An Integrated Assessment. (Washington, D.C.: National Science and Technology Council, Committee on Environmental and Natural Resources, 2005: 8, 12). http://www.esrl.noaa.gov/csd/AQRS/reports/napapreport05.pdf.

${ }^{70}$ Carlson, Curtis, Dallas Burtraw, Maureen Cropper, and Karen L. Palmer. 2000. "Sulfur Dioxide Control by Electric Utilities: What Are the Gains from Trade?" Journal of Political Economy 108, no. 6 (2000): 1313 Table 2.

doi:10.1086/317681.

${ }^{71}$ Chan, G. et al. The $\mathrm{SO}_{2}$ Allowance Trading System and the Clean Air Act Amendments of 1990: Reflections on Twenty Years of Policy Innovation. (Cambridge, MA: Harvard Kennedy School, 2012).

${ }^{72}$ Stavins, Robert N. "What can we learn from the grand policy experiment? Lessons from $\mathrm{SO}_{2}$ allowance trading." The Journal of Economic Perspectives. 12, no. 3 (1998): 71.

${ }^{73}$ Stavins, Robert N. "What can we learn from the grand policy experiment? Lessons from $\mathrm{SO}_{2}$ allowance trading." The Journal of Economic Perspectives. 12, no. 3 (1998): 71.

${ }^{74}$ Chan, H. Ron, B. Andrew Chupp, Maureen Cropper, and Nicholas Z. Muller. "The Net Benefits of the Acid Rain Program: What Can We Learn from the Grand Policy Experiment?" Resources For the Future, DP 15-25, 2015: 4. http://www.rff.org/files/sharepoint/Worklmages/Download/RFF-DP-15-25.pdf.

${ }^{75}$ Burtraw, Dallas. "Cost Savings, Market Performance, and Economic Benefits of the U.S. Acid Rain Program." Washington, DC: Resources for the Future. Discussion Paper 98-28 REV. 1998.

${ }^{76}$ Chan, H. Ron, B. Andrew Chupp, Maureen Cropper, and Nicholas Z. Muller. The Net Benefits of the Acid Rain Program: What Can We Learn from the Grand Policy Experiment?. Resources For the Future, DP 15-25, 2015: 4.

${ }^{77}$ Carlson, Curtis, Dallas Burtraw, Maureen Cropper, and Karen L. Palmer. 2000. "Sulfur Dioxide Control by Electric Utilities: What Are the Gains from Trade?" Journal of Political Economy 108, no. 6 (2000): 1292-1326.

${ }^{78}$ Ellerman, A. Denny. "Markets for clean air: The US acid rain program." Cambridge University Press, 2000.

${ }^{79}$ White, Keith. " $\mathrm{SO}_{2}$ Compliance and Allowance Trading: Developments and Outlook," prepared for the Electric Power Research Institute (EPRI), EPRI TR-107897 (1997). http://www.epri.com/abstracts/Pages/ProductAbstract.aspx?Productld=TR-107897.

${ }^{80}$ White, Keith D., Energy Ventures Analysis, Inc., and Van Horn Consulting. 1995. "The Emission Allowance Market and Electric Utility $\mathrm{SO}_{2}$ Compliance in a Competitive and Uncertain Future," prepared for the Electric Power Research Institute, EPRI TR-105490s (1995): Table 1-1, page 1-8., High Utilization Scenario.

http://www.epri.com/abstracts/Pages/ProductAbstract.aspx?Productld=TR-105490\&Mode=download

${ }^{81}$ Government Accounting Office (GAO). "Air Pollution: Allowance Trading Offers an Opportunity to Reduce Emissions at Less Cost," GAO/RCED-95-30, Washington, D.C. 1994.

${ }^{82}$ Van Horn Consulting and Keith D. White. "Integrated Analysis of Fuel, Technology and Emission Allowance Markets," prepared for the Electric Power Research Institute, EPRI TR-102510 (1993). http://www.epri.com/abstracts/Pages/ProductAbstract.aspx?Productld=TR-102510.

${ }^{83}$ Environmental Protection Agency. "Regulatory Impact Analysis of the Final Acid Rain Implementation Regulations." Washington, DC: EPA (1992). Cited in Chan et al. (2015): 4.

${ }^{84}$ Carlson, Curtis, Dallas Burtraw, Maureen Cropper, and Karen L. Palmer. 2000. "Sulfur Dioxide Control by Electric Utilities: What Are the Gains from Trade?" Journal of Political Economy 108, no. 6 (2000): 1318 Table 3, page 1318, actual compliance cost.

${ }^{85}$ Ellerman, A. Denny, Richard Schmalensee, Paul L. Joskow, Juan Pablo Montero, and Elizabeth M. Bailey.

"Emissions Trading under the U.S. Acid Rain Program: Evaluation of Compliance Costs and Allowance Market

Performance." (Cambridge, Mass.: MIT Center for Energy and Environmental Policy Research). 1997: 36, Table 9. http://citeseerx.ist.psu.edu/viewdoc/download?doi=10.1.1.212.966\&rep=rep1\&type=pdf.

${ }^{86}$ Portney, Paul R. "Policy watch: economics and the Clean Air Act." The Journal of Economic Perspectives. 4, no. 4 (1990): 176. http://pubs.aeaweb.org/doi/pdfplus/10.1257/jep.4.4.173

${ }^{87}$ Burtraw, Dallas, Alan Krupnick, Erin Mansur, Daivd Austin, and Deirdre Farrell. "Costs and benefits of reducing air pollutants related to acid rain." Contemporary Economic Policy 16, no. 4 (1998): 379. Figure 1, page 388. http://onlinelibrary.wiley.com/doi/10.1111/j.1465-7287.1998.tb00527.x/abstract.

${ }_{88}^{8}$ Portney, Paul R. "Policy watch: economics and the Clean Air Act." (1990): 175. http://pubs.aeaweb.org/doi/pdfplus/10.1257/jep.4.4.173 


\footnotetext{
${ }^{89}$ National Acid Precipitation Assessment Program, 1989 Report to the President and Congress, 1990.

90 Portney, Paul R. "Policy watch: economics and the Clean Air Act." (1990): 175.

http://pubs.aeaweb.org/doi/pdfplus/10.1257/jep.4.4.173

${ }^{91}$ Chan, G. et al. The $\mathrm{SO}_{2}$ Allowance Trading System and the Clean Air Act Amendments of 1990: Reflections on Twenty Years of Policy Innovation.

${ }^{92}$ Muller, N.Z. and R.O. Mendelsohn. "Measuring the damages of air pollution in the United States." Journal of Environmental Economics and Management 54, no. 1 (2007): 8.

${ }^{93}$ Environmental Protection Agency. "National Emission Standards for Hazardous Air Pollutants from Coal- and OilFired Electric Utility Steam Generating Units and Standards of Performance for Fossil-Fuel-Fired Electric Utility, Industrial-Commercial-Institutional, and Small Industrial-Commercial-Institutional Steam Generating Units;" Technical Correction, Signed on 3/17/2016 and available at https://www3.epa.gov/airquality/powerplanttoxics/pdfs/20160317fr.pdf.

${ }^{94}$ EPA. "Regulatory Finding on the Emissions of Hazardous Air Pollutants from Electric Utility Steam Generating Units." December 2000. AD-FRL-6919-6. https://www3.epa.gov/airquality/powerplanttoxics/utilfind.pdf. Accessed May 4, 2016.

${ }^{95}$ EPA. "Regulatory Impact Analysis for the Final Mercury and Air Toxics Standards." p. 1-6, Table 1-1. December 2011. http://www3.epa.gov/mats/actions.html. Accessed February 17, 2016.

96 EPA. "Regulatory Impact Analysis for the Final Mercury and Air Toxics Standards." Table ES-2, p. ES-2. December 2011. http://www3.epa.gov/mats/actions.html. Accessed February 17, 2016.

${ }^{97}$ EPA. "Regulatory Impact Analysis for the Final Mercury and Air Toxics Standards." Table ES-2 and Figure ES-1. December 2011. http://www3.epa.gov/mats/actions.html. Accessed February 17, 2016.

${ }^{98}$ National Research Council. “Energy Research at DOE: Was It Worth It?" p 177-182.

http://www.nap.edu/catalog/10165.htm. Accessed May 23, 2016.

${ }_{99}$ Bezdek, R.H. and Wendling, R.M. "The return on investment of the clean coal technology program in the USA."

Energy Policy 54 (2013) 104-112. http://dx.doi.org/10.1016/i.enpol.2012.10.076.

100 Environmental Protection Agency. "Summary of Coal Unit Controls: 2015."

https://www.epa.gov/airmarkets/summary-coal-unit-controls-2015. Accessed May 4, 2016.

${ }^{101}$ EPA. "Emissions Tracking Highlights" Table of Coal Unit Characteristics: 2015. https://www.epa.gov/sites/production/files/2016-05/coalunitcharacteristics2015.xls. Accessed May 17, 2016.

${ }^{102}$ Environmental Protection Agency. "Emissions Tracking Highlights." https://www.epa.gov/airmarkets/emissionstracking-highlights. Accessed May 4, 2016.

${ }^{103}$ National Research Council. "Energy Research at DOE: Was It Worth It?” p 177

http://www.nap.edu/catalog/10165.htm. Accessed May 23, 2016.

${ }^{104}$ Bezdek, R.H. and Wendling, R.M. "The return on investment of the clean coal technology program in the USA." Energy Policy 54 (2013) 104-112. http://dx.doi.org/10.1016/j.enpol.2012.10.076.

${ }^{105}$ DOE. "Clean Coal Technology: From Research to Reality." http://energy.gov/fe/downloads/clean-coaltechnology-research-reality. Accessed March 23, 2016.

${ }^{106}$ Bezdek, R.H. and Wendling, R.M. "The return on investment of the clean coal technology program in the USA." Energy Policy 54 (2013) 104-112. http://dx.doi.org/10.1016/i.enpol.2012.10.076.

107 EPA 2015. “Coal-fired Characteristics and Controls: 2015." Table of Coal Unit Characteristics. https://www.epa.gov/airmarkets/emissions-tracking-highlights. Accessed June 2, 2016.

${ }^{108}$ Energy Information Administration. "Short-Term Energy Outlook." (2016): Table 7a: U.S. Electricity Industry Overview. http://www.eia.gov/forecasts/steo/. Accessed February 2, 2016.

${ }^{109}$ EPSA Base Case scenario, Electric Power Sector Emissions. Analysis conducted by the DOE Office of Energy Policy and Systems Analysis (EPSA), February 2016.

${ }^{110}$ EPSA Base Case scenario, Electric Power Sector Emissions. Analysis conducted by the DOE Office of Energy Policy and Systems Analysis (EPSA), February 2016.

${ }^{111}$ Analysis conducted by the DOE Office of Energy Policy and Systems Analysis (EPSA), February 2016.

112 Projections analysis conducted by the DOE Office of Energy Policy and Systems Analysis (EPSA), February 2016.

${ }^{113} \mathrm{SO}_{2}$ and $\mathrm{NO}_{x}$ historical emissions from EPA. "Air Pollutant Emissions Trends Data."

https://www.epa.gov/sites/production/files/2015-07/national tier1 caps.xlsx. Accessed June 1, 2016.
} 
${ }^{114}$ Mercury historical emissions 1990-2005 from EPA. "Report on the Environment: Mercury Emissions." https://cfpub.epa.gov/roe/download/051 mercury-emissions exh-1.csv. Accessed June 1, 2016. Mercury historical emissions in 2008 and 2011 from EPA. "National Emissions Inventory 2008 and 2011." https://www.epa.gov/air-emissions-inventories/2011-national-emissions-inventory-nei-data and https://www.epa.gov/air-emissions-inventories/2008-national-emissions-inventory-nei-data. Accessed June 1, 2016.

${ }^{115}$ Byun, Daewon W. and J.K.S. Ching. "Science Algorithms of the EPA Models-3 Community Multiscale Air Quality (CMAQ) Modeling System." 1999. http://nepis.epa.gov/Adobe/PDF/30003R9Y.PDF. Accessed March 17, 2016.

${ }^{116}$ Environmental Protection Agency. https://www.epa.gov/fera/human-exposure-modeling-general.

${ }^{117}$ Environmental Protection Agency. "BenMAP-CE - An Open-Source Platform to Quantify the Health Impacts and Economic Value of Stressors." Briefing for Office of Water (2013).

https://www.epa.gov/sites/production/files/2014-12/documents/benmapcedemoslides.pdf.

${ }_{118}$ Muller, N.Z., R.O. Mendelsohn., and W.D. Nordhaus. "Environmental accounting for pollution in the United States economy." The American Economic Review 101, no. 5 (2011): 1649-1675.

${ }^{119}$ Muller, N.Z. and R.O. Mendelsohn. "Measuring the damages of air pollution in the United States." Journal of Environmental Economics and Management 54, no. 1 (2007): 1-14.

${ }^{120}$ Industrial Economics. "Health and Welfare Benefits Analyses to Support the Second Section 812 Benefit-Cost Analysis of the Clean Air Act." (Cambridge, MA: Industrial Economics, 2011).

${ }^{121}$ Environmental Protection Agency. "Regulatory Impact Analysis for the Final Revisions to the National Ambient Air Quality Standards for Particulate Matter." Office of Air Quality Planning and Standards - Health and Environmental Impacts Division, EPA-452/R-12-005, (2012): Table 5-2. https://www3.epa.gov/ttn/ecas/regdata/RIAs/finalria.pdf.

${ }_{122}$ Brunekreef, B. and B. Forsberg. 2005. "Epidemiological evidence of effects of coarse airborne particles on health." European Respiratory Journal 26, no. 2 (2005): 309-318. doi: 10.1183/09031936.05.00001805.

${ }^{123}$ Pope III, C. Arden and Douglas W Dockery. 2006. "Health effects of fine particulate air pollution: lines that connect." Journal of the air \& waste management association 56, no. 6 (2006): 709-742.

${ }^{124}$ Environmental Protection Agency. Progress Cleaning the Air and Improving People's Health. 2015. https://www.epa.gov/clean-air-act-overview/progress-cleaning-air-and-improving-peoples-health.

${ }^{125}$ Environmental Protection Agency. "National Emissions Inventory, version 2, Technical Support Document." Edited by Office of Air Quality Planning and Standards Environmental Protection Agency, Air Quality Assessment Division, Emissions Inventory and Analysis Group. Research Triangle Park, North Carolina, 2015.

${ }^{126}$ Industrial Economics. "Health and Welfare Benefits Analyses to Support the Second Section 812 Benefit-Cost Analysis of the Clean Air Act."

${ }^{127}$ The following provide a list of the most relevant studies used in air pollution impact studies: Muller and Mendelsohn (2007); NRC (2010); Bell, Michelle L., Richard D. Morgenstern, and Winston Harrington. "Quantifying the human health benefits of air pollution policies: Review of recent studies and new directions in accountability research." Environmental Science \& Policy 14, no. 4 (2011): 357-368; IEC (2011); Muller, Mendelsohn, and Nordhaus (2011); Ščasný, Milan et al. 2015. "Quantifying the Ancillary Benefits of the Representative Concentration Pathways on Air Quality in Europe." Environmental and Resource Economics 62, no. 2 (2015): 383415. doi: 10.1007/s10640-015-9969-y.

128 Lee, E.H. and W.E. Hogsett. "Methodology for calculating inputs for ozone secondary standard benefits analysis: Part II." Prepared for EPA, Office of Air Quality Planning and Standards. 1996.

${ }^{129}$ Menz, Fredric C. and Hans M Seip. "Acid rain in Europe and the United States: an update." Environmental Science \& Policy 7, no. 4 (2004): 253-265.

${ }^{130}$ Fiscus, Edwin L, Fitzgerald L Booker, and Kent O Burkey. 2005. "Crop responses to ozone: uptake, modes of action, carbon assimilation and partitioning." Plant, Cell \& Environment 28, no. 8 (2005): 997-1011.

${ }^{131}$ Van Dingenen, Rita et al. "The global impact of ozone on agricultural crop yields under current and future air quality legislation." Atmospheric Environment 43, no. 3 (2009): 604-618.

${ }^{132}$ Environmental Protection Agency. Benefits and Costs of the Clean Air Act 1990-2020, the Second Prospective Study. (Washington, DC: Environmental Protection Agency, Office of Air and Radiation, 2011). 
${ }^{133}$ Industrial Economics. "Health and Welfare Benefits Analyses to Support the Second Section 812 Benefit-Cost Analysis of the Clean Air Act."

${ }^{134}$ Industrial Economics. "Health and Welfare Benefits Analyses to Support the Second Section 812 Benefit-Cost Analysis of the Clean Air Act.

135 Industrial Economics. "Health and Welfare Benefits Analyses to Support the Second Section 812 Benefit-Cost Analysis of the Clean Air Act.

${ }^{136}$ Industrial Economics. "Health and Welfare Benefits Analyses to Support the Second Section 812 Benefit-Cost Analysis of the Clean Air Act.

${ }^{137}$ Industrial Economics. "Health and Welfare Benefits Analyses to Support the Second Section 812 Benefit-Cost Analysis of the Clean Air Act.

${ }^{138}$ National Atmospheric Deposition Program. 2015 Summary of Critical Load Maps. edited by Critical Loads Atmospheric Deposition Science Committee: National Atmospheric Deposition Program. 2015.

${ }^{139}$ National Atmospheric Deposition Program. 2015 Summary of Critical Load Maps.

${ }^{140}$ Porter, E., T.F. Blett, D.U. Potter, and C. Huber. 2005. "Protecting resources on federal lands: implications of critical loads for atmospheric deposition of nitrogen and sulfur." Bioscience 55, 603-612. http://www.nature.nps.gov/air/pubs/pdf/2005CriticalLoadBioSci.pdf. Accessed June 2, 2016.

${ }^{141}$ United Nations Environment Programme. "Opportunities to Limit Near-Term Climate Change: An Integrated Assessment of Black Carbon and Tropospheric Ozone and Its Precursors." Nairobi: United Nations Environment Programme and World Meteorological Organization, 2011.

${ }^{142}$ Sundseth, Kyrre et al. "Economic benefits from decreased mercury emissions: Projections for 2020." Journal of Cleaner Production 18, no. 4 (2010): 386-394.

143 Weil et al. "Blood mercury levels and neurobehavioral function." Jama 293, no. 15 (2005):1875-1882.

${ }^{144}$ National Research Council 2000. "Toxicological Effects of Methylmercury." DOI: 10.17226/9899. Accessed June 2, 2016.

${ }^{145}$ Keeney, Ralph L. and Howard Raiffa. Multicriteria analysis is an alternative evaluation method to compare impacts on different sectors and from different pollutants: Decisions with multiple objectives: preferences and value trade-offs. (Cambridge University Press, 1993).

${ }^{146}$ Federal Register. Executive Order 12866 of September 30, 1993 “Regulatory Planning and Review." Vol. 58, No. 190 (1993). https://www.whitehouse.gov/sites/default/files/omb/inforeg/eo12866/eo12866 10041993.pdf. 147 Muller, Nicholas Z. and Robert Mendelsohn. "Measuring the damages of air pollution in the United States." Journal of Environmental Economics and Management 54, no. 1 (2007): 1-14. http://dx.doi.org/10.1016/i.jeem.2006.12.002.

${ }^{148}$ Muller, Nicholas Z. and Robert Mendelsohn. "Measuring the damages of air pollution in the United States." Journal of Environmental Economics and Management 54, no. 1 (2007): 1-14.

http://dx.doi.org/10.1016/j.jeem.2006.12.002.

${ }^{149}$ Muller, Nicholas Z., Robert Mendelsohn, and William Nordhaus. "Environmental accounting for pollution in the United States economy." The American Economic Review (2011): 1649-1675.

http://www.jstor.org/stable/23045618.

${ }^{150}$ Muller, Nicholas Z. and Robert Mendelsohn. "Measuring the damages of air pollution in the United States." Journal of Environmental Economics and Management 54, no. 1 (2007): 1-14. doi:10.1016/j.jeem.2006.12.002

${ }^{151}$ Fann, N., C.M. Fulcher and B.J. Hubbell. "The influence of location, source, and emission type in estimates of the human health benefits of reducing a ton of air pollution." Air Qual Atmos Health, 2 (2009):169-176. http://link.springer.com/article/10.1007/s11869-009-0044-0.

152 Muller, Nicholas Z., Robert Mendelsohn, and William Nordhaus. "Environmental accounting for pollution in the United States economy." The American Economic Review (2011): 1649-1675.

http://www.jstor.org/stable/23045618.

${ }^{153}$ Chan, H.R. et al. "The Net Benefits of the Acid Rain Program. What Can We Learn from the Grand Policy Experiment?" Resources For the Future, RFF DP (2015): 15-25.

154 Jaramillo, Paulina and Nicholas Z. Muller. "Air pollution emissions and damages from energy production in the US: 2002-2011." Energy Policy 90 (2016): 202-211. 
${ }^{155}$ Muller, Nicholas Z. and Robert Mendelsohn. "Measuring the damages of air pollution in the United States." Journal of Environmental Economics and Management 54, no. 1 (2007): 1-14. Page 10.

http://dx.doi.org/10.1016/i.jeem.2006.12.002.

${ }^{156}$ National Research Council (NRC). Committee on Health, Environmental, Other External Costs, Benefits of Energy Production, and Consumption. 2010. Hidden costs of energy: unpriced consequences of energy production and use. National Academies Press (2010).

http://www.nap.edu/login.php?record id=12794\&page=http\%3A\%2F\%2Fwww.nap.edu\%2Fdownload.php\%3Frec ord id\%3D12794.

${ }^{157}$ Muller, Nicholas Z. and Robert Mendelsohn. "Efficient pollution regulation: getting the prices right." The American Economic Review (2009): 1714-1739. http://www.jstor.org/stable/25592534.

158 Muller, Nicholas Z., Robert Mendelsohn, and William Nordhaus. "Environmental accounting for pollution in the United States economy." The American Economic Review (2011): 1649-1675.

http://www.jstor.org/stable/23045618.

${ }^{159}$ Viscusi, W Kip and Joseph E Aldy. "The value of a statistical life: a critical review of market estimates throughout the world." Journal of risk and uncertainty 27, no. 1 (2003): 5-76.

${ }^{160}$ EPA webpage. "Mortality Risk Valuation." https://www.epa.gov/environmental-economics/mortality-riskvaluation. Accessed June 2, 2016.

${ }^{161}$ Aldy, J.E. and W.K. Viscusi 2006. "Adjusting the Value of a Statistical Life for Age and Cohort Effects." Resources for the Future. http://www.rff.org/files/sharepoint/Worklmages/Download/RFF-DP-06-19.pdf. Accessed June 2, 2016.

162 Mendelsohn, Robert. "An economic analysis of air pollution from coal-fired power plants." Journal of Environmental Economics and Management 7, no. 1 (1980):30-43.

163 Ottinger, Richard L. et al. "Environmental costs of electricity." (New York, NY: Oceana Publications, 1990).

${ }^{164}$ Krupnick, Alan J. and Dallas Burtraw. 1996. "The social costs of electricity: Do the numbers add up?" Resource and Energy Economics 18, no. 4 (1996): 423-466. Doi: http://dx.doi.org/10.1016/S0928-7655(97)00023-7.

165 National Research Council (NRC). Committee on Health, Environmental, Other External Costs, Benefits of Energy Production, and Consumption. 2010. Hidden costs of energy: unpriced consequences of energy production and use. National Academies Press (2010).

http://www.nap.edu/login.php?record id=12794\&page=http\%3A\%2F\%2Fwww.nap.edu\%2Fdownload.php\%3Frec ord id\%3D12794.

${ }^{166}$ National Research Council (NRC). Committee on Health, Environmental, Other External Costs, Benefits of Energy Production, and Consumption. 2010. Hidden costs of energy: unpriced consequences of energy production and use. National Academies Press (2010). Page 92.

http://www.nap.edu/login.php?record id=12794\&page=http\%3A\%2F\%2Fwww.nap.edu\%2Fdownload.php\%3Frec ord id\%3D12794.

${ }^{167}$ Muller, Nicholas Z., Robert Mendelsohn, and William Nordhaus. "Environmental accounting for pollution in the United States economy." The American Economic Review (2011): 1649-1675. Table 5, p. 1670.

http://www.jstor.org/stable/23045618.

168 Muller, Nicholas Z. and Robert Mendelsohn. "Measuring the damages of air pollution in the United States." Journal of Environmental Economics and Management 54, no. 1 (2007): 1-14.

http://dx.doi.org/10.1016/i.jeem.2006.12.002.

169 Jaramillo, Paulina and Nicholas Z. Muller. "Air pollution emissions and damages from energy production in the US: 2002-2011." Energy Policy 90 (2016): 202-211. Table 1, page 206.

http://www.sciencedirect.com/science/article/pii/S0301421515302494.

170 Jaramillo, Paulina, and Nicholas Z. Muller. "Air pollution emissions and damages from energy production in the US: 2002-2011." Energy Policy 90 (2016): 202-211.

http://www.sciencedirect.com/science/article/pii/S0301421515302494.

${ }_{171}$ Muller, Nicholas Z. and Robert Mendelsohn. "Measuring the damages of air pollution in the United States." Journal of Environmental Economics and Management 54, no. 1 (2007): 1-14.

http://dx.doi.org/10.1016/i.jeem.2006.12.002. 
172 Jaramillo, Paulina and Nicholas Z. Muller. "Air pollution emissions and damages from energy production in the US: 2002-2011." Energy Policy 90 (2016): 202-211. Table 1, page 206.

http://www.sciencedirect.com/science/article/pii/S0301421515302494.

173 Jaramillo, Paulina and Nicholas Z. Muller. "Air pollution emissions and damages from energy production in the US: 2002-2011." Energy Policy 90 (2016): 202-211. Table 1, page 206.

${ }^{174}$ Fann, Neal, Kirk R. Baker, and Charles M. Fulcher. "Characterizing the PM 2.5-related health benefits of emission reductions for 17 industrial, area and mobile emission sectors across the US." Environment international 49 (2012): 141-151.

${ }^{175}$ National Research Council, Committee on Health, Environmental, Other External Costs, Benefits of Energy Production, and Consumption. 2010. Page 92.

${ }^{176}$ Muller, Nicholas Z., Robert Mendelsohn, and William Nordhaus. "Environmental accounting for pollution in the United States economy." The American Economic Review (2011): 1649-1675. Table 5, p. 1670.

http://www.jstor.org/stable/23045618.

${ }^{177}$ Muller, Nicholas Z. and Robert Mendelsohn. "Measuring the damages of air pollution in the United States." Journal of Environmental Economics and Management 54, no. 1 (2007): 1-14. http://dx.doi.org/10.1016/j.jeem.2006.12.002.

178 Jaramillo, Paulina and Nicholas Z. Muller. "Air pollution emissions and damages from energy production in the US: 2002-2011." Energy Policy 90 (2016): 202-211. Table 1, page 206.

${ }^{179}$ Fann, Neal, Charles M. Fulcher, and Bryan J. Hubbell. "The influence of location, source, and emission type in estimates of the human health benefits of reducing a ton of air pollution." Air Quality, Atmosphere \& Health 2, no. 3 (2009): 169-176.

${ }^{180}$ Fann, Neal, Kirk R. Baker, and Charles M. Fulcher. "Characterizing the PM 2.5-related health benefits of emission reductions for 17 industrial, area and mobile emission sectors across the US." Environment international 49 (2012): 141-151.

${ }^{181}$ Levy, Jonathan I., Lisa K. Baxter, and Joel Schwartz. "Uncertainty and variability in health-related damages from coal-fired power plants in the United States." Risk Analysis 29, no. 7 (2009): 1000-1014.

${ }^{182}$ Buonocore, Jonathan J., Xinyi Dong, John D. Spengler, Joshua S. Fu, and Jonathan I. Levy. "Using the Community Multiscale Air Quality (CMAQ) model to estimate public health impacts of PM 2.5 from individual power plants."

Environment international 68 (2014): 200-208.

183 Muller, Nicholas Z., Robert Mendelsohn, and William Nordhaus. "Environmental accounting for pollution in the United States economy." The American Economic Review (2011): 1649-1675. http://www.jstor.org/stable/23045618.

${ }^{184}$ National Research Council (NRC). Committee on Health, Environmental, Other External Costs, Benefits of Energy Production, and Consumption. 2010. Hidden costs of energy: unpriced consequences of energy production and use. National Academies Press (2010).

http://www.nap.edu/login.php?record id=12794\&page=http\%3A\%2F\%2Fwww.nap.edu\%2Fdownload.php\%3Frec ord id\%3D12794.

${ }^{185}$ National Research Council (NRC). Committee on Health, Environmental, Other External Costs, Benefits of Energy Production, and Consumption. 2010. Hidden costs of energy: unpriced consequences of energy production and use. National Academies Press (2010). Page 93.

http://www.nap.edu/login.php?record id=12794\&page=http\%3A\%2F\%2Fwww.nap.edu\%2Fdownload.php\%3Frec ord id\%3D12794.

${ }^{186}$ National Research Council (NRC). Committee on Health, Environmental, Other External Costs, Benefits of Energy Production, and Consumption. 2010. Hidden costs of energy: unpriced consequences of energy production and use. National Academies Press (2010). Page 93.

http://www.nap.edu/login.php?record id=12794\&page=http\%3A\%2F\%2Fwww.nap.edu\%2Fdownload.php\%3Frec ord id\%3D12794.

187 Muller, Nicholas Z., Robert Mendelsohn, and William Nordhaus. "Environmental accounting for pollution in the United States economy." The American Economic Review (2011): 1649-1675. Pages 1669-1670.

${ }^{188}$ Muller, Nicholas Z., Robert Mendelsohn, and William Nordhaus. "Environmental accounting for pollution in the United States economy." The American Economic Review (2011): 1649-1675. Pages 1669-1670. 
${ }^{189}$ National Research Council (NRC). Committee on Health, Environmental, Other External Costs, Benefits of Energy Production, and Consumption. 2010. Hidden costs of energy: unpriced consequences of energy production and use. National Academies Press (2010). Table 2-15, page 118.

http://www.nap.edu/login.php?record_id=12794\&page=http\%3A\%2F\%2Fwww.nap.edu\%2Fdownload.php\%3Frec ord id\%3D12794.

${ }^{190}$ National Research Council (NRC). Committee on Health, Environmental, Other External Costs, Benefits of Energy Production, and Consumption. 2010. Hidden costs of energy: unpriced consequences of energy production and use. National Academies Press (2010). Page 119.

http://www.nap.edu/login.php?record id=12794\&page=http\%3A\%2F\%2Fwww.nap.edu\%2Fdownload.php\%3Frec ord id\%3D12794.

${ }^{191}$ National Research Council (NRC). Committee on Health, Environmental, Other External Costs, Benefits of Energy Production, and Consumption. 2010. Hidden costs of energy: unpriced consequences of energy production and use. National Academies Press (2010). Table 2-15, page 118.

http://www.nap.edu/login.php?record id=12794\&page=http\%3A\%2F\%2Fwww.nap.edu\%2Fdownload.php\%3Frec ord id\%3D12794.

192 National Research Council (NRC). Committee on Health, Environmental, Other External Costs, Benefits of Energy Production, and Consumption. 2010. Hidden costs of energy: unpriced consequences of energy production and use. National Academies Press (2010).

http://www.nap.edu/login.php?record_id=12794\&page=http\%3A\%2F\%2Fwww.nap.edu\%2Fdownload.php\%3Frec ord id\%3D12794.

${ }_{193}$ Muller, Nicholas Z., Robert Mendelsohn, and William Nordhaus. "Environmental accounting for pollution in the United States economy." The American Economic Review (2011): 1649-1675. Pages 1669-1670.

http://www.jstor.org/stable/23045618.

${ }^{194}$ Dockery, Douglas W. et al. "An association between air pollution and mortality in six US cities." New England Journal of Medicine 329 no. 24 (1993): 1753-1759.

195 Pope III, C. Arden, Richard T. Burnett, Michael J. Thun, Eugenia E. Calle, Daniel Krewski, Kazuhiko Ito, and George D. Thurston. "Lung cancer, cardiopulmonary mortality, and long-term exposure to fine particulate air pollution." Jama 287, no. 9 (2002): 1132-1141. http://jama.jamanetwork.com/article.aspx?articleid=194704. ${ }^{196}$ Krewski, D., Jerrett, M., Burnett, R.T., Ma, R., Hughes, E., Shi, Y., Turner, M.C., Pope 3rd, C.A., Thurston, G., Calle, E.E. and Thun, M.J., 2009. Extended follow-up and spatial analysis of the American Cancer Society study linking particulate air pollution and mortality. Research report (Health Effects Institute), (140), p.5. http://www.ncbi.nlm.nih.gov/pubmed/19627030.

${ }^{197}$ Laden, Francine, Joel Schwartz, Frank E. Speizer, and Douglas W. Dockery. "Reduction in fine particulate air pollution and mortality: extended follow-up of the Harvard Six Cities study." American journal of respiratory and critical care medicine 173, no. 6 (2006): 667-672. http://www.atsjournals.org/doi/abs/10.1164/rccm.200503$\underline{4430 C .}$.

${ }^{198}$ Muller, Nicholas Z., Robert Mendelsohn, and William Nordhaus. "Environmental accounting for pollution in the United States economy." The American Economic Review (2011): 1649-1675. Table 5, p. 1670.

http://www.jstor.org/stable/23045618.

199 Jaramillo, Paulina and Nicholas Z. Muller. "Air pollution emissions and damages from energy production in the US: 2002-2011." Energy Policy 90 (2016): 202-211. Table 1, page 206. http://www.sciencedirect.com/science/article/pii/S0301421515302494.

${ }^{200}$ National Research Council (NRC). Committee on Health, Environmental, Other External Costs, Benefits of Energy Production, and Consumption. 2010. Hidden costs of energy: unpriced consequences of energy production and use. National Academies Press (2010). P. 92.

http://www.nap.edu/login.php?record id=12794\&page=http\%3A\%2F\%2Fwww.nap.edu\%2Fdownload.php\%3Frec ord id\%3D12794.

${ }^{201}$ Fann, Neal, Kirk R. Baker, and Charles M. Fulcher. "Characterizing the PM 2.5-related health benefits of emission reductions for 17 industrial, area and mobile emission sectors across the US." Environment international 49 (2012): 141-151. http://dx.doi.org/10.1016/j.envint.2012.08.017. 
202 Levy, Jonathan I., Lisa K. Baxter, and Joel Schwartz. "Uncertainty and variability in health-related damages from coal-fired power plants in the United States." Risk Analysis 29, no. 7 (2009): 1000-1014. P. 1008. http://onlinelibrary.wiley.com/doi/10.1111/i.1539-6924.2009.01227.x/full.

${ }^{203}$ Muller, Nicholas Z., Robert Mendelsohn, and William Nordhaus. "Environmental accounting for pollution in the United States economy." The American Economic Review (2011): 1649-1675. Table 5, p. 1670. http://www.jstor.org/stable/23045618.

${ }^{204}$ National Research Council (NRC). Committee on Health, Environmental, Other External Costs, Benefits of Energy Production, and Consumption. 2010. Hidden costs of energy: unpriced consequences of energy production and use. National Academies Press (2010). Table 2.9, p. 92.

http://www.nap.edu/login.php?record id=12794\&page=http\%3A\%2F\%2Fwww.nap.edu\%2Fdownload.php\%3Frec ord id\%3D12794.

${ }^{205}$ National Research Council (NRC). Committee on Health, Environmental, Other External Costs, Benefits of Energy Production, and Consumption. 2010. Hidden costs of energy: unpriced consequences of energy production and use. National Academies Press (2010). Table 2.15, p. 118.

http://www.nap.edu/login.php?record id=12794\&page=http\%3A\%2F\%2Fwww.nap.edu\%2Fdownload.php\%3Frec ord id\%3D12794.

${ }^{206}$ National Research Council (NRC). Committee on Health, Environmental, Other External Costs, Benefits of Energy Production, and Consumption. 2010. Hidden costs of energy: unpriced consequences of energy production and use. National Academies Press (2010). Table 2.9, p. 92.

http://www.nap.edu/login.php?record id=12794\&page=http\%3A\%2F\%2Fwww.nap.edu\%2Fdownload.php\%3Frec ord id\%3D12794.

${ }^{207}$ National Research Council (NRC). Committee on Health, Environmental, Other External Costs, Benefits of Energy Production, and Consumption. 2010. Hidden costs of energy: unpriced consequences of energy production and use. National Academies Press (2010). Table 2.15, p. 118.

http://www.nap.edu/login.php?record id=12794\&page=http\%3A\%2F\%2Fwww.nap.edu\%2Fdownload.php\%3Frec ord id\%3D12794.

${ }^{208}$ Muller, Nicholas Z., Robert Mendelsohn, and William Nordhaus. "Environmental accounting for pollution in the United States economy." The American Economic Review (2011): 1649-1675. Page 2. http://www.jstor.org/stable/23045618.

${ }^{209}$ Muller, Nicholas Z., Robert Mendelsohn, and William Nordhaus. "Environmental accounting for pollution in the United States economy." The American Economic Review (2011): 1649-1675. Page 2. http://www.jstor.org/stable/23045618.

${ }^{210}$ Fann, N., C.M. Fulcher and B.J. Hubbell. 2009. "The influence of location, source, and emission type in estimates of the human health benefits of reducing a ton of air pollution." Air Qual Atmos Health, 2:169-176. Figure 4, p. 174. http://link.springer.com/article/10.1007/s11869-009-0044-0.

${ }^{211}$ Fann, Neal, Kirk R. Baker, and Charles M. Fulcher. "Characterizing the PM 2.5-related health benefits of emission reductions for 17 industrial, area and mobile emission sectors across the US." Environment international 49 (2012): 141-151. P. 145. http://dx.doi.org/10.1016/i.envint.2012.08.017.

${ }^{212}$ Levy, Jonathan I., Lisa K. Baxter, and Joel Schwartz. "Uncertainty and variability in health-related damages from coal-fired power plants in the United States." Risk Analysis 29, no. 7 (2009): 1000-1014. P. 1008. http://onlinelibrary.wiley.com/doi/10.1111/i.1539-6924.2009.01227.x/full.

${ }^{213}$ Buonocore, Jonathan J., Xinyi Dong, John D. Spengler, Joshua S. Fu, and Jonathan I. Levy. "Using the Community Multiscale Air Quality (CMAQ) model to estimate public health impacts of PM 2.5 from individual power plants."

Environment international 68 (2014): 200-208. P. 204. http://dx.doi.org/10.1016/i.envint.2014.03.031.

${ }^{214}$ Jaramillo, Paulina and Nicholas Z. Muller. "Air pollution emissions and damages from energy production in the US: 2002-2011." Energy Policy 90 (2016): 202-211.Table S2, Supporting Information.

http://www.sciencedirect.com/science/article/pii/S0301421515302494.

${ }^{215}$ Fann, Neal, Charles M. Fulcher, and Bryan J. Hubbell. "The influence of location, source, and emission type in estimates of the human health benefits of reducing a ton of air pollution." Air Quality, Atmosphere \& Health 2, no. 3 (2009): 169-176. http://link.springer.com/article/10.1007\%2Fs11869-009-0044-0. 
${ }^{216}$ Fann, Neal, Charles M. Fulcher, and Bryan J. Hubbell. "The influence of location, source, and emission type in estimates of the human health benefits of reducing a ton of air pollution." Air Quality, Atmosphere \& Health 2, no. 3 (2009): 169-176. Page 169. http://link.springer.com/article/10.1007\%2Fs11869-009-0044-0.

${ }^{217}$ Fann, Neal, Charles M. Fulcher, and Bryan J. Hubbell. "The influence of location, source, and emission type in estimates of the human health benefits of reducing a ton of air pollution." Air Quality, Atmosphere \& Health 2, no. 3 (2009): 169-176. Figure 4, p. 174. http://link.springer.com/article/10.1007\%2Fs11869-009-0044-0.

${ }^{218}$ Environmental Protection Agency. "Regulatory Impact Analysis for the Clean Power Plan Final Rule" EPA-452/R15-003, August 2015.

${ }^{219}$ Environmental Protection Agency. "Regulatory Impact Analysis for the Clean Power Plan Final Rule" EPA-452/R15-003, August 2015. Table 4-7, p. 4-23. https://www.epa.gov/sites/production/files/2015-08/documents/cppfinal-rule-ria.pdf.

${ }^{220}$ National Research Council (NRC). Committee on Health, Environmental, Other External Costs, Benefits of Energy Production, and Consumption. 2010. Hidden costs of energy: unpriced consequences of energy production and use. National Academies Press (2010).

http://www.nap.edu/login.php?record id=12794\&page=http\%3A\%2F\%2Fwww.nap.edu\%2Fdownload.php\%3Frec ord id\%3D12794.

${ }^{221}$ National Research Council (NRC). Committee on Health, Environmental, Other External Costs, Benefits of Energy Production, and Consumption. 2010. Hidden costs of energy: unpriced consequences of energy production and use. National Academies Press (2010).

http://www.nap.edu/login.php?record id=12794\&page=http\%3A\%2F\%2Fwww.nap.edu\%2Fdownload.php\%3Frec ord id\%3D12794.

222 Intergovernmental Panel on Climate Change. Climate change 2001: mitigation. Contribution of Working Group III to the Third Assessment Report of the IPCC. (Cambridge, MA: Cambridge University Press, 2001).

${ }^{223}$ Environmental Protection Agency. "Regulatory Impact Analysis for the Proposed Carbon Pollution Guidelines for Existing Power Plants and Emission Standards for Modified and Reconstructed Power Plants."

${ }^{224}$ Environmental Protection Agency. "Regulatory Impact Analysis for the Clean Power Plan Final Rule" EPA-452/R15-003, August 2015. Table ES-8, p. ES-21. https://www.epa.gov/sites/production/files/2015-08/documents/cppfinal-rule-ria.pdf.

${ }^{225}$ Environmental Protection Agency. "Regulatory Impact Analysis for the Clean Power Plan Final Rule" EPA-452/R15-003, August 2015. https://www.epa.gov/sites/production/files/2015-08/documents/cpp-final-rule-ria.pdf.

${ }^{226}$ National Research Council (NRC). Committee on Health, Environmental, Other External Costs, Benefits of Energy Production, and Consumption. 2010. Hidden costs of energy: unpriced consequences of energy production and use. National Academies Press (2010).

http://www.nap.edu/login.php?record id=12794\&page=http\%3A\%2F\%2Fwww.nap.edu\%2Fdownload.php\%3Frec ord id\%3D12794.

${ }^{227}$ Muller, N.Z., R.O. Mendelsohn., and W.D. Nordhaus. "Environmental accounting for pollution in the United States economy." 2011. Table 5, p. 1670. http://www.jstor.org/stable/23045618.

${ }^{228}$ Groosman B., N.Z. Muller, and E. O'Neill-Toy. "The ancillary benefits from climate policy in the United States."

${ }^{229}$ Interagency Working Group on Social Cost of Carbon. 2015. Technical Support Document: Technical Update of the Social Cost of Carbon for Regulatory Impact Analysis Under Executive Order 12866, May 2013, revised July 2015. Table 2, p. 13. https://www.whitehouse.gov/sites/default/files/omb/inforeg/scc-tsd-final-july-2015.pdf ${ }^{230}$ National Research Council (NRC). Committee on Health, Environmental, Other External Costs, Benefits of Energy Production, and Consumption. 2010. Hidden costs of energy: unpriced consequences of energy production and use. National Academies Press (2010). Table 7-3, p. 361.

http://www.nap.edu/login.php?record id=12794\&page=http\%3A\%2F\%2Fwww.nap.edu\%2Fdownload.php\%3Frec ord id\%3D12794.

${ }^{231}$ Thompson, Tammy M., Sebastian Rausch, Rebecca K. Saari, and Noelle E. Selin. "A systems approach to evaluating the air quality co-benefits of US carbon policies." Nature Climate Change 4, no. 10 (2014): 917-923. Page 919. 
${ }^{232}$ West, J.J., S.J. Smith, R.A. Silva, V. Naik, Y. Zhang, Z. Adelman, M.M. Fry, S. Anenberg, L.W. Horowitz, and J-F Lamarque. "Co-benefits of mitigating global greenhouse gas emissions for future air quality and human health." Nature Climate Change, 3, 2013, Figure 4 p. 888.

${ }^{233}$ Environmental Protection Agency. "Regulatory Impact Analysis for the Clean Power Plan Final Rule" EPA-452/R15-003, August 2015. Table ES-8, p. ES-21. https://www.epa.gov/sites/production/files/2015-08/documents/cppfinal-rule-ria.pdf.

${ }^{234}$ Burtraw, D., A. Krupnick, K. Palmer, A. Paul, M. Toman, and C. Bloyd. "Ancillary benefits of reduced air pollution in the US from moderate greenhouse gas mitigation policies in the electricity sector." Journal of Environmental Economics and Management 45 (2003): 650-673. P. 650.

${ }^{235}$ Nemet, G.F., T. Holloway, and P. Meier. "Implications of incorporating air-quality co-benefits into climate change policymaking." Environmental Research Letters, 5 (2010), p. 2.

${ }^{236}$ Groosman B., N.Z. Muller, and E. O'Neill-Toy. "The ancillary benefits from climate policy in the United States." Environmental and Resource Economics (2011) 50: 585-603, p. 599.

${ }^{237}$ West, J.J., S.J. Smith, R.A. Silva, V. Naik, Y. Zhang, Z. Adelman, M.M. Fry, S. Anenberg, L.W. Horowitz, and J-F Lamarque. "Co-benefits of mitigating global greenhouse gas emissions for future air quality and human health." Nature Climate Change, 3, 2013, p. 886.

${ }^{238}$ Department of Energy. "Addressing Environmental Aspects of TS\&D Infrastructure" QER Report: Energy Transmission, Storage, and Distribution Infrastructure. (2015): pp. 7-3. April 2016. http://www.energy.gov/epsa/quadrennial-energy-review-first-installment. 239 Department of Energy. “Quadrennial Energy Review: Energy Transmission, Storage and Distribution Infrastructure." p 7-3 - 7-6. April 2016. http://www.energy.gov/epsa/quadrennial-energy-review-first-installment ${ }^{240}$ Department of Energy. “Quadrennial Energy Review: Energy Transmission, Storage and Distribution Infrastructure." p 9-1 - 9-24. April 2016. http://www.energy.gov/epsa/quadrennial-energy-review-first-installment ${ }^{241}$ White House, 2015. "Modernizing our Electric transmission Infrastructure and Driving the Development of Clean Energy." July, 2015 Report. https://www.whitehouse.gov/blog/2015/07/21/modernizing-our-electrictransmission-infrastructure-and-driving-development-clean-e.

${ }^{242}$ National Renewable Energy Laboratory. (2012). Renewable Electricity Futures Study. Hand, M.M.; Baldwin, S.; DeMeo, E.; Reilly, J.M.; Mai, T.; Arent, D.; Porro, G.; Meshek, M.; Sandor, D. eds. 4 vols. NREL/TP-6A20-52409. Golden, CO: National Renewable Energy Laboratory. http://www.nrel.gov/analysis/re futures/. Table A-10, p. A66.

${ }^{243}$ EURELECTRIC, 2011. "Life Cycle Assessment of Electricity Generation." WG Environmental Management \& Economics. Page. 14. http://www.eurelectric.org/media/26740/report-Ica-resap-final-2011-420-0001-01-e.pdf. ${ }^{244}$ Fthenakis, V., and H.-Y. Kim. "Land Use and Electricity Generation: A Life -cycle Analysis." Renewable and Sustainable Energy Reviews 13, no. 6-7 (2009): 1465-1474. http://www.sciencedirect.com/science/article/pii/S1364032108001354. Accessed May 2016

${ }^{245}$ Marcy, C., J. Logan, J. McCall, F. Flores-Espino, A. Bloom, J. Aabakken, W. Cole, T. Jenkins, and G. Porro, "Electricity Generation Baseline Report," National Renewable Energy Lab (2016).

${ }^{246}$ Brown, Marilyn A., Daniel D'Arcy, Melissa Lapsa, Isha Sharma, Yufei Li. Solid Waste from the Operation and Decommissioning of Power Plants, Oak Ridge National Laboratory, ORNL/SPR-2016/774 (2016).

${ }^{247}$ Fthenakis, V., and H.-Y. Kim. "Land Use and Electricity Generation: A Life -cycle Analysis." Renewable and Sustainable Energy Reviews 13, no. 6-7 (2009): 1465-1474. Page 1466.

${ }^{248}$ Sovacool, B.K. 2008. Valuing the greenhouse gas emissions from nuclear power: A critical survey. Energy Policy 36(8):2950-2963. Available at:

http://www.biol.uw.edu.pl/pl/files/docs/st dokt/SD SCB Bird and bat mortalities.pdf. Accessed June 3, 2016. ${ }^{249}$ Skone, T., J. Littlefield, J. Marriott, G. Cooney, M. Jamieson, J. Hakian, and G. Schivley. 2014. Life Cycle Analysis of Natural Gas Extraction and Power Generation. DOE/NETL-2014/1646.

http://www.netl.doe.gov/File\%20Library/Research/Energy\%20Analysis/Life\%20Cycle\%20Analysis/NETL-NG-PowerLCA-29May2014.pdf

${ }^{250}$ Fthenakis, V., and H.-Y. Kim. "Land Use and Electricity Generation: A Life -cycle Analysis." Renewable and Sustainable Energy Reviews 13, no. 6-7 (2009): 1465-1474. Figure 2.

http://www.sciencedirect.com/science/article/pii/S1364032108001354. Accessed May 2016. 
${ }^{251}$ Fthenakis, V., and H.-Y. Kim. "Land Use and Electricity Generation: A Life -cycle Analysis." Renewable and Sustainable Energy Reviews 13, no. 6-7 (2009): 1465-1474. See page 1468.

http://www.sciencedirect.com/science/article/pii/S1364032108001354. Accessed May 2016.

$252 \mathrm{NEI}, 2015$. Land Requirements for Carbon-Free Technologies.

http://www.nei.org/CorporateSite/media/filefolder/Policy/Papers/Land Use Carbon Free Technologies.pdf?ext= .pdf.

${ }^{253}$ Fthenakis, V., and H.-Y. Kim. "Land Use and Electricity Generation: A Life -cycle Analysis." Renewable and Sustainable Energy Reviews 13, no. 6-7 (2009): 1465-1474.

http://www.sciencedirect.com/science/article/pii/S1364032108001354. Accessed May 2016.

${ }^{254}$ Brown, Marilyn A., Daniel D'Arcy, Melissa Lapsa, Isha Sharma, Yufei Li. Solid Waste from the Operation and Decommissioning of Power Plants, Oak Ridge National Laboratory, ORNL/SPR-2016/774 (2016).

${ }^{255}$ Sovacool, B.K. 2008. Valuing the greenhouse gas emissions from nuclear power: A critical survey. Energy Policy 36(8):2950-2963. Available at:

http://www.biol.uw.edu.pl/pl/files/docs/st dokt/SD SCB Bird and bat mortalities.pdf. Accessed June 3, 2016.

${ }^{256}$ Department of Energy. “Wind Vision: A new era for Wind Power In the United States," March 2015. http://www.energy.gov/sites/prod/files/WindVision Report final.pdf

${ }^{257}$ Fthenakis, V., and H.-Y. Kim. 2009. "Land Use and Electricity Generation: A Life -cycle Analysis. 2009.

258 Denholm, P. et al. Land-Use Requirements of Modern Wind Power Plants in the United States. 2009 Page 22.

259 Department of Energy. "Wind Vision: A new era for Wind Power In the United States," March 2015. http://www.energy.gov/sites/prod/files/WindVision_Report final.pdf

260 Department of Energy. "Wind Vision: A New Era for Wind Power in the United States," 2015.

http://www.energy.gov/sites/prod/files/WindVision_Report_final.pdfhttp://www.energy.gov/sites/prod/files/Win dVision Report final.pdf.

${ }^{261}$ Department of Energy. "Wind Vision: A new era for Wind Power In the United States," March 2015. See table 2-

8. http://www.energy.gov/sites/prod/files/WindVision Report final.pdf. Accessed June 3, 2016.

262 Department of Energy. "Wind Vision: A New Era for Wind Power in the United States," 2015. Accessed

05/04/2016, Page 97, Online: http://www.energy.gov/sites/prod/files/WindVision Report final.pdf.

${ }^{263}$ American Wind Wildlife Institute (AWWI), "Wind Turbine Interactions with Wildlife and their Habitats, January

2014, Accessed 05/04/2016, Page:4, https://awwi.org/wp-content/uploads/2014/05/AWWI-Wind-Wildlife-

Interactions-Factsheet-05-27-14.pdf.

${ }^{264}$ Ellenbogen, J.M. et al. "Wind Turbine Health Impact Study: Report of Independent Expert Panel." Prepared for the Massachusetts Department of Environmental Protection and the Massachusetts Department of Public Health. 2012: 164 pp. http://www.vpirg.org/resources/wind-turbine-health-impact-study-report-of-independent-expertpanel/http://www.vpirg.org/resources/wind-turbine-health-impact-study-report-of-independent-expert-panel/.

265 Minnesota Department of Health (MDH) Environmental Health Division. "Public Health Impacts of Wind

Turbines." 2009; 32 pp.

http://www.health.state.mn.us/divs/eh/hazardous/topics/windturbines.pdhttp://www.health.state.mn.us/divs/e h/hazardous/topics/windturbines.pdf.

${ }^{266}$ Parsons Brinckehoff “Update of UK Shadow Flicker Evidence Base." Prepared for UK Department of Energy and Climate Change (DECC). 2011.

https://www.gov.uk/government/uploads/system/uploads/attachment data/file/48052/1416-update-uk-shadowflicker-evidence-base.pdf.

${ }^{267}$ Hoen, B. et al. "Spatial Hedonic Analysis of the Effects of Wind Energy Facilities on Surrounding Property Values in the United States." LBNL-6362E. Berkeley, CA: Lawrence Berkeley National Laboratory, 2013. http://escholarship.org/uc/item/5gx7k135.

${ }^{268}$ Atkinson-Palombo, C. and B. Hoen.. "Relationship Between Wind Turbines and Residential Property Values in Massachusetts." LBNL/6371E. Berkeley, CA: Lawrence Berkeley National Laboratory, 2014.

http://emp.lbl.gov/publications/relationship-between-wind-turbines-andresidential-property-valuesmassachusetts.

${ }^{269}$ Ellenbogen, J.M. et al. "Wind Turbine Health Impact Study: Report of Independent Expert Panel." 
270 Ong, S., C. Campbell, P. Denholm, R. Margolis, and G. Heath. 2013. Land-Use Requirements for Solar Power Plants in the United States. Technical Report NREL/TP-6A20-56290. Executive Summary.

http://www.nrel.gov/docs/fy13osti/56290.pdf. Accessed May, 2016.

271 Ong, S., C. Campbell, P. Denholm, R. Margolis, and G. Heath. 2013. Land-Use Requirements for Solar Power Plants in the United States. Technical Report NREL/TP-6A20-56290. Table ES-1.

http://www.nrel.gov/docs/fy13osti/56290.pdf. Accessed May, 2016..

272 Massachusetts Institute of Technology. 2015. "The Future of Solar Energy." 2015.

273 Massachusetts Institute of Technology. 2015. "The Future of Solar Energy." 2015. Page 129

${ }^{274}$ National Renewable Energy Labratory. 2016. Rooftop Solar Photovoltaic Technical Potential in the United States: A Detailed Assessment. NREL/TP-6A20-65298. ASee page 34.

http://www.nrel.gov/docs/fy16osti/65298.pdf. Accessed June 3, 2016.

${ }^{275}$ Rebecca A. Kagan, Tabitha C. Viner, Pepper W. Trail, and Edgard O. Espinoza National Fish and Wildlife Forensics Laboratory, "Avian Mortality at Solar Energy Facilities in Southern California: A Preliminary Analysis," Online:

http://docketpublic.energy.ca.gov/PublicDocuments/09-AFC-

07C/TN202538 20140623T154647 Exh 3107 Kagan et al 2014.pdf.

${ }^{276}$ Palen Solar Power Project - Compliance" Docketpublic.energy.ca.gov Online:

http://docketpublic.energy.ca.gov/PublicDocuments/09-AFC-

07C/TN201977 20140407T161504 Center Supplemental Opposition to Motion.pdf Page 1.

277 Cassandra Sweet, "High-Tech Solar Projects Fail to Deliver," The Wall Street Journal, June 12, 2015, Accessed 04 May 2016, Online http://www.wsj.com/articles/high-tech-solar-projects-fail-to-deliver-

1434138485\#:S3o /QqZ4E6bYA.

${ }^{278}$ Manville, Albert. M II. "Bird Strikes and Electrocutions at Power Lines, Communication Towers, and Wind Turbines: State of the Art and State of the Science - Next Steps Toward Mitigation." USDA Forest Service 1052. 2005. http://www.fs.fed.us/psw/publications/documents/psw gtr191/Asilomar/pdfs/1051-1064.pdf. Accessed May 16, 2016 Page 1055.

279 Loss, S., Will, T., and Marra, P. "The impact of free-ranging domestic cats on wildlife of the United States." Nature Communications 4, Article 1396. December 2012.

http://www.nature.com/ncomms/journal/v4/n1/full/ncomms2380.html See Abstract.

${ }^{280}$ Department of Energy. "GEOTHERMAL POWER PLANTS - MINIMIZING LAND USE AND IMPACT." Geothermal. 2016. http://energy.gov/eere/geothermal/geothermal-power-plants-minimizing-land-use-and-impact. Accessed May 162016.

${ }^{281}$ Department of Energy. "GEOTHERMAL POWER PLANTS - MINIMIZING LAND USE AND IMPACT." Geothermal. 2016. http://energy.gov/eere/geothermal/geothermal-power-plants-minimizing-land-use-and-impact. Accessed May 162016.

282 Department of Energy. "GEOTHERMAL POWER PLANTS - MINIMIZING LAND USE AND IMPACT." Geothermal. 2016. http://energy.gov/eere/geothermal/geothermal-power-plants-minimizing-land-use-and-impact. Accessed May 162016.

283 Department of Energy. "GEOTHERMAL POWER PLANTS - MINIMIZING LAND USE AND IMPACT." Geothermal. 2016. http://energy.gov/eere/geothermal/geothermal-power-plants-minimizing-land-use-and-impact. Accessed May 162016.

${ }^{284}$ Department of Energy. "GEOTHERMAL POWER PLANTS - MINIMIZING LAND USE AND IMPACT." Geothermal. 2016. http://energy.gov/eere/geothermal/geothermal-power-plants-minimizing-land-use-and-impact. Accessed May 162016.

285 Department of Energy. "GEOTHERMAL POWER PLANTS - MINIMIZING LAND USE AND IMPACT." Geothermal. 2016. http://energy.gov/eere/geothermal/geothermal-power-plants-minimizing-land-use-and-impact. Accessed May 162016.

286 Union of Concerned Scientists. "Environmental Impacts of Geothermal Energy." Renewable Energy. Nd. http://www.ucsusa.org/clean energy/our-energy-choices/renewable-energy/environmental-impacts-geothermalenergy.htm|\#.VzpWZJErK01. Accessed May 16, 2016.

${ }^{287}$ Fthenakis, V., and H.-Y. Kim. "Land Use and Electricity Generation: A Life -cycle Analysis." Renewable and Sustainable Energy Reviews 13, no. 6-7 (2009): 1465-1474. Table 7. 
${ }^{288}$ Foundation for Water \& Energy Education. "Changes to the Ecosystem." http://fwee.org/environment/how-ahydroelectric-project-can-affect-a-river/changes-to-the-ecosystem/." Accessed April 5, 2016

289 Union of Concerned Scientists. "Environmental Impacts of Hydroelectric Power."

http://www.ucsusa.org/clean_energy/our-energy-choices/renewable-energy/environmental-impacts-

hydroelectric-power.html\#.VwQTkulrLcs

290 U.S. Fish \& Wildlife Service. "Hydropower Environmental Issues."

http://www.fws.gov/habitatconservation/hydro issues.pdf. Accessed April 5, 2016.

${ }^{291}$ Environment and Ecology. "Hydropower and the Environment." Energy and the Environment. 2016.

http://environment-ecology.com/energy-and-environment/100-hydropower-and-the-environment.html. Accessed May 16, 2016.

292 Foundation for Water \& Energy Education. "Changes to the Ecosystem."

${ }^{293}$ Arvesen, Anders et al. 2015. "Life cycle assessment of transport of electricity via different voltage levels: A case study for Nord-Trondelag County in Norway." Applied Energy 157 (2015): 144-151.

${ }^{294} \mathrm{Wu}$, Grace C., Margaret S.Torn, and James H. Williams. "Incorporating Land-Use Requirements and

Environmental Constraints in Low-Carbon Electricity Planning for California." Environmental Science \& Technology 49, no. 4 (2015): 2013-2021.

295 Parfomak, Paul W. Physical Security of the U.S. Power Grid: High-Voltage Transformer Substations. (Washington, DC: Congressional Research Service, 2014).

${ }^{296}$ Harris Williams \& Co. “Transmission \& Distribution Infrastructure." 2014.

http://www.harriswilliams.com/sites/default/files/industry reports/ep td white_paper $06 \quad 10 \quad 14$ final.pdf. Accessed April 7, 2016.

${ }^{297}$ Araneo, Rodolfo et al. "Low-Environmental Impact Routeing of Overhead Power Lines for the Connection of Renewable Energy Plants to the Italian HV Grid." In Conference Proceedings: $201414^{\text {th }}$ International Conference On Environment And Electrical Engineering (EEEIC) (2014): 386-391.

${ }^{298}$ Conniff, Richard. "Electric Power Rights of Way: A New Frontier for Conservation." Yale Environment 360. 2014. http://e360.yale.edu/feature/electric power rights of way a new frontier for conservation/2816/.

${ }^{299}$ Public Service Commission of Wisconsin. "Environmental Impacts of Transmission Lines." Publications. 2013.

Page 6. http://psc.wi.gov/thelibrary/publications/electric/electric10.pdf. Accessed May 24, 2016.

300 Public Service Commission of Wisconsin. "Underground Electric Transmission Lines." Publications. 2011. Page

17. https://psc.wi.gov/thelibrary/publications/electric/electric11.pdf. Accessed May 24, 2016.

301 Public Service Commission of Wisconsin. "Underground Electric Transmission Lines." Publications. 17 (2011). https://psc.wi.gov/thelibrary/publications/electric/electric11.pdf. Accessed May 24, 2016.

${ }^{302}$ Cain, Nicholas L. and Hal T. Nelson. "What drives opposition to high voltage transmission lines?" Land Use Policy 33 (2013): 204-213.

${ }^{303}$ Schively, C. "Understanding the NIMBY and LULU phenomena: reassessing our knowledge base and informing future research." Journal of Planning Literature 21 no. 3 (2007): 255-266.

${ }^{304}$ North American Electric Reliability Corporation. "2012 Long-Term Reliability Assessment." November 2012. 36.

http://www.nerc.com/news/Headlines\%20DL/2012\%20Long\%20Term\%20Reliability\%20Assessment.pdf. Accessed May 17, 2016.

${ }^{305}$ Electric Power Research Institute. “Distributed Resource Cost Impacts on T\&D Systems." 2001. 24.

http://www.epri.com/abstracts/Pages/ProductAbstract.aspx?Productld $=000000000001003975$

${ }^{306}$ Electric Power Research Institute. "Distributed Resource Cost Impacts on T\&D Systems." 2001.

http://www.epri.com/abstracts/Pages/ProductAbstract.aspx?Productld=0000000000001003975

${ }^{307}$ Federal Energy Regulatory Commission. "The potential benefit of DG and rate-related issues that may impede

their expansion." 2007. https://www.ferc.gov/legal/fed-sta/exp-study.pdf

${ }^{308}$ Department of Energy. "QER Report: Energy Transmission, Storage, and Distribution Infrastructure." 2015.

http://energy.gov/sites/prod/files/2015/07/f24/ElectricityAppendix.pdf, Accessed June 10, 2016., Appendix C, p. 33.

${ }^{309}$ Energy Information Administration. Frequency Asked Questions. 2016.

http://www.eia.gov/tools/faqs/faq.cfm?id=105\&t=3 
${ }^{310}$ Jorge, R.S. and E.G. Hertwich. "Environmental evaluation of power transmission in Norway." Applied Energy 101 (2014): 513-520, Special Issue: SI.

${ }^{311}$ Electric Power Research Institute. "T\&D and ROW Environmental Issues - Program 51," 2014, Online: http://mydocs.epri.com/docs/Portfolio/P2014/2014_P051.pdf.

312 Rioux, Sebastien, Jean-Pierre L. Savard, and Alyssa A. Gerick. "Avian mortalities due to transmission line collisions: a review of current estimates and field methods with an emphasis on applications to the Canadian electric network." Avian Conservation and Ecology 8, no. 2 (2013): Article Number: 7 Online: http://www.aceeco.org/vol8/iss2/art7/ See Abstract.

${ }^{313}$ Rioux, Sebastien, Jean-Pierre L. Savard, and Alyssa A. Gerick. 2013.

${ }^{314}$ North American Electric Reliability Corporation. "Protection System Maintenance." 2007. http://www.nerc.com/docs/pc/spctf/Relay Maintenance Tech Ref approved by PC.pdf

${ }^{315}$ Conniff, Richard. "Electric Power Rights of Way: A New Frontier for Conservation." Yale Environment 360. 2014. http://e360.yale.edu/feature/electric power rights of way a new frontier for conservation/2816/

${ }^{316}$ North American Electric Reliability Corporation. “Protection System Maintenance." 2007.

${ }^{317}$ Bureau of Land Management. "Memorandum of Understanding Among The Edison Electric Institute and the U.S. Department of Agriculture Forest Service and the U.S. Department of the Interior Bureau of Land Management, Fish and Wildlife Service, and National Park Service, and the U.S. Environmental Protection Agency." May 2006. http://www.fs.fed.us/specialuses/documents/eei-mou-3-3-06.rtf. Accessed July 1, 2016.

${ }^{318}$ National Institute of Environmental Health Sciences. "Electric and Magnetic Fields Associated with the Use of Electric Power." 2002.

http://www.niehs.nih.gov/health/materials/electric and magnetic fields associated with the use of electric $p$ ower questions and answers english 508.pdf

${ }^{319}$ Huang, Jiongli et al. "Association between Exposure to Electromagnetic Fields from High Voltage Transmission Lines and Neurobehavioral Function in Children." PLOS ONE 8 no. 7 (2013): Article Number: e67284.

${ }^{320}$ National Institute of Environmental Health Sciences. 2002.

${ }^{321}$ Department of Energy. “Quadrennial Energy Review: Energy Transmission, Storage and Distribution Infrastructure." p 9-1 - 9-24. April 2016. http://www.energy.gov/epsa/quadrennial-energy-review-first-installment 322 White House, 2015. "Modernizing our Electric transmission Infrastructure and Driving the Development of Clean Energy." July, 2015 Report. https://www.whitehouse.gov/blog/2015/07/21/modernizing-our-electrictransmission-infrastructure-and-driving-development-clean-e.

${ }^{323}$ Department of Energy. "Quadrennial Energy Review: Energy Transmission, Storage and Distribution Infrastructure." p 9-4. April 2016. http://www.energy.gov/epsa/quadrennial-energy-review-first-installment ${ }^{324}$ Federal Energy Regulatory Commission. "A Guide to the FERC Electric Transmission Facilities Permit Process." Guides 2010. http://www.ferc.gov/resources/guides/electric/guide-transmission.pdf. Accessed March 29, 2016. ${ }^{325}$ Holtkamp, James A. and Mark A Davidson. "Transmission Siting in the Western United States: Overview and Recommendations Prepared as Information to the Western Interstate Energy Board." 2009. https://www.hollandhart.com/articles/transmission siting white_paper final.pdf. Accessed 30 March, 2016. ${ }^{326}$ Federal Energy Regulatory Commission. "A Guide to the FERC Electric Transmission Facilities Permit Process." Guides. 2010. http://www.ferc.gov/resources/guides/electric/guide-transmission.pdf. Accessed March 29, 2016. ${ }^{327}$ Department of Energy. "Siting and Permitting of TS\&D Infrastructure." QER Report: Energy Transmission, Storage, and Distribution Infrastructure. 2015. http://energy.gov/sites/prod/files/2015/08/f25/QER\%20Chapter\%20IX\%20Siting\%20and\%20Permitting\%20April\% 202015.pdf. Accessed March 29, 2016.

${ }^{328}$ Department of Energy. "Siting and Permitting of TS\&D Infrastructure." QER Report: Energy Transmission, Storage, and Distribution Infrastructure. 2015. http://energy.gov/sites/prod/files/2015/08/f25/QER\%20Chapter\%20IX\%20Siting\%20and\%20Permitting\%20April\% 202015.pdf. Accessed March 29, 2016.

${ }^{329}$ Government Accountability Office. "National Environmental Policy Act: Little Information Exists on NEPA Analyses." 2014. http://www.gao.gov/assets/670/662546.pdf. Accessed April 1, 2016. 
${ }^{330}$ Holtkamp, James A. and Mark A Davidson. "Transmission Siting in the Western United States: Overview and Recommendations Prepared as Information to the Western Interstate Energy Board." 2009.

https://www.hollandhart.com/articles/transmission siting white paper final.pdf. Accessed 30 March, 2016.

${ }^{331}$ Department of Energy. "Siting and Permitting of TS\&D Infrastructure." QER Report: Energy Transmission, Storage, and Distribution Infrastructure. 2015.

http://energy.gov/sites/prod/files/2015/08/f25/QER\%20Chapter\%20IX\%20Siting\%20and\%20Permitting\%20April\% 202015.pdf. Accessed March 29, 2016.

${ }^{332}$ Department of Energy. "Siting and Permitting of TS\&D Infrastructure." QER Report: Energy Transmission, Storage, and Distribution Infrastructure. 2015.

http://energy.gov/sites/prod/files/2015/08/f25/QER\%20Chapter\%20IX\%20Siting\%20and\%20Permitting\%20April\% 202015.pdf. Accessed March 29, 2016.

${ }^{333}$ Department of Energy. "Siting and Permitting of TS\&D Infrastructure." QER Report: Energy Transmission, Storage, and Distribution Infrastructure. 2015.

http://energy.gov/sites/prod/files/2015/08/f25/QER\%20Chapter\%20IX\%20Siting\%20and\%20Permitting\%20April\% 202015.pdf. Accessed March 29, 2016.

${ }^{334}$ Department of Energy. “Quadrennial Energy Review: Energy Transmission, Storage and Distribution Infrastructure." p 9-4. April 2016. http://www.energy.gov/epsa/quadrennial-energy-review-first-installment

${ }^{335}$ Department of Energy. "Quadrennial Energy Review: Energy Transmission, Storage and Distribution Infrastructure." p 7-4 - 7-5. April 2016. http://www.energy.gov/epsa/quadrennial-energy-review-first-installment. ${ }^{336}$ Department of Energy. “Quadrennial Energy Review: Energy Transmission, Storage and Distribution Infrastructure." p 9-8. April 2016. http://www.energy.gov/epsa/quadrennial-energy-review-first-installment 33740 C.F.R. $\S 1503$ and $\S \S 1501.4(\mathrm{e})$ and 1506.6.

${ }^{338}$ Center for Rural Affairs. "Landowner Compensation in Transmission Siting for Renewable Energy Facilities Siting." 2014. http://www.cfra.org/sites/www.cfra.org/files/publications/landowner-compensation-052014 0.pdf. Accessed February 25, 2015.

${ }^{339}$ Zichella, C. and J. Hladik. “America's Power Plan - Siting: Finding a Home for Renewable Energy and Transmission." 2013. http://americaspowerplan.com/wp-content/uploads/2013/09/APP-SITING-PAPER.pdf. Accessed February 10, 2015.

${ }^{340}$ Western Governors' Association. "Siting and Permitting Transmission Reforms." (2012): 4. http://westgov.org/component/docman/doc download/1608-transmission-recommendations-2012. Accessed February 26, 2015.

${ }^{341}$ Department of Energy. "Quadrennial Energy Review: Energy Transmission, Storage, and Distribution Infrastructure." p 7-6. April 2015. http://www.energy.gov/epsa/quadrennial-energy-review-first-installment. 342 White House, 2015. "Modernizing our Electric transmission Infrastructure and Driving the Development of Clean Energy." July, 2015 Report. https://www.whitehouse.gov/blog/2015/07/21/modernizing-our-electrictransmission-infrastructure-and-driving-development-clean-e.

${ }^{343}$ White House, 2015. "Modernizing our Electric transmission Infrastructure and Driving the Development of Clean Energy." July, 2015 Report. https://www.whitehouse.gov/blog/2015/07/21/modernizing-our-electrictransmission-infrastructure-and-driving-development-clean-e.

${ }^{344}$ Department of Energy. 2015. "Siting and Permitting of TS\&D Infrastructure." QER Report: Energy Transmission, Storage, and Distribution Infrastructure. (2015): 9-12-9-13.

http://energy.gov/sites/prod/files/2015/08/f25/QER\%20Chapter\%20IX\%20Siting\%20and\%20Permitting\%20April\% 202015.pdf. Accessed March 29, 2016.

${ }^{345}$ U.S. Fish \& Wildlife Service. "Land-Based Wind Energy Guidelines." http://www.fws.gov/ecologicalservices/energy-development/wind.html. Accessed, June 2, 2016.

${ }^{346}$ U.S. Fish \& Wildlife Service. "Eagle Conservation Plan Guidance Module 1 - Land-based Wind Energy Version 2." https://www.fws.gov/migratorybirds/pdf/management/eagleconservationplanguidance.pdf. Accessed June 2, 2016.

347 U.S. Fish \& Wildlife Service Endangered Species. "Indiana Bat: Section 7 and Section 10 Guidance for Wind Energy Projects." 2011. http://www.fws.gov/midwest/endangered/mammals/inba/WindEnergyGuidance.html. 
${ }^{348}$ Energy Information Administration. "Hydropower generators produce clean electricity, buy hydropower does have environmental impacts." 2015. http://www.eia.gov/Energyexplained/?page=hydropower environment. Accessed April 5, 2016.

${ }^{349}$ Department of Energy. Bats and Wind Energy Cooperative. http://energy.gov/eere/wind/downloads/featuredpublications-bats-and-wind-energy-cooperative. Accessed June 2, 2016.

350 Pacific Northwest National Laboratory. Tethys. http://tethys.pnnl.gov/about-tethys. Accessed June 2, 2016.

${ }^{351}$ National Renewable Energy Laboratory. Wind-Wildlife Impacts Literature Database. https://wild.nrel.gov/.

Accessed June 2, 2016.

352136 FERC, ๆ 61,051, at p. 155.

353130 FERC ๆ 61,056, (2010): 43.

${ }^{354}$ Southworth, Katie. 2016. "Sustainable FERC Project: Southeastern Regional Transmission Planning Update," Presentation at February 11, 2016 meeting of the National Association of State Energy Officials, Washington, DC.

355 GridSolar, LLC, 2015, 2015 Annual Report Boothbay Sub-Region Smart Grid Reliability Pilot Project, Docket No. 2011-138, October 28., http://www.scotthemplinglaw.com/files/attachments/maine interim report boothbay smart grid reliability pil ot project.pdf. Accessed April 2, 2016.

${ }^{356}$ Neme, Chris and Rich Sedano. "US Experience with Efficiency as a Transmission and Distribution System Resource." (2012): iii. Regulatory Assistance Project Report. http://www.raponline.org/document/download/id/4765 Accessed May 2, 2016.

${ }^{357}$ Sovacool, B.K. The Dirty Energy Dilemma: What's Blocking Clean Power in the United States. (ABC-CLIO, 2008).

${ }^{358}$ National Research Council, Committee on Health, Environmental, Other External Costs, Benefits of Energy Production, and Consumption. Hidden costs of energy: unpriced consequences of energy production and use. (National Academies Press, 2010). DOI: 10.17226/12794

${ }^{359}$ EPA. "Steam Electric Power Generating Effluent Guidelines." https://www.epa.gov/eg/steam-electric-powergenerating-effluent-guidelines. Accessed April 11, 2016.

${ }^{360}$ Brown, Marilyn A., Daniel D'Arcy, Melissa Lapsa, Isha Sharma, Yufei Li. Solid Waste from the Operation and Decommissioning of Power Plants, Oak Ridge National Laboratory, ORNL/SPR-2016/774 (2016).

361 EPA. "Steam Electric Effluent Guidelines - 2015 Final Rule; Fact Sheet."

https://www.epa.gov/sites/production/files/2015-10/documents/steam-electric-final-rule-factsheet 10-012015.pdf. Accessed April 11, 2016.

362 EPA. "Steam Electric Effluent Guidelines - 2015 Final Rule; Fact Sheet." https://www.epa.gov/sites/production/files/2015-10/documents/steam-electric-final-rule-factsheet 10-012015.pdf. Accessed April 11, 2016.

${ }^{363}$ EPA 821-R-09-008. "Steam Electric Power Generating Point Source Category: Final Detailed Study Report." p xii. October 2009. https://www.epa.gov/eg/steam-electric-power-generating-effluent-guidelines-backgrounddocuments. Accessed April 20, 2016.

${ }^{364}$ EPA. "Steam Electric Power Generating Effluent Guidelines - 2015 Final Rule." https://www.epa.gov/eg/steamelectric-power-generating-effluent-guidelines-2015-final-rule. Accessed April 20, 2016.

${ }^{365}$ EPA 821-R-09-008. "Steam Electric Power Generating Point Source Category: Final Detailed Study Report." p xii. October 2009. https://www.epa.gov/eg/steam-electric-power-generating-effluent-guidelines-backgrounddocuments. Accessed April 20, 2016.

${ }^{366}$ Federal Register 40 CFR Part 423. "Effluent Limitations Guidelines and Standards for the Steam Electric Power Generating Point Source Category; Final Rule." p. 67846-67847. https://www.gpo.gov/fdsys/pkg/FR-2015-1103/pdf/2015-25663.pdf. Accessed April 20, 2016.

${ }^{367}$ EPA 821-R-09-008. “Steam Electric Power Generating Point Source Category: Final Detailed Study Report." p 321. October 2009. https://www.epa.gov/eg/steam-electric-power-generating-effluent-guidelines-backgrounddocuments. Accessed April 20, 2016.

${ }^{368}$ EPA 821-R-09-008. "Steam Electric Power Generating Point Source Category: Final Detailed Study Report." Table 3-7, p 3-22. October 2009. https://www.epa.gov/eg/steam-electric-power-generating-effluent-guidelinesbackground-documents. Accessed April 20, 2016. 
${ }^{369}$ EPA 821-R-09-008. "Steam Electric Power Generating Point Source Category: Final Detailed Study Report." p 312 through 3-32 and p 7-1 through 7-10. October 2009. https://www.epa.gov/eg/steam-electric-powergenerating-effluent-guidelines-background-documents. Accessed April 20, 2016.

${ }^{370}$ EPA 821-R-09-008. "Steam Electric Power Generating Point Source Category: Final Detailed Study Report." p 318 through 3-19. October 2009. https://www.epa.gov/eg/steam-electric-power-generating-effluent-guidelinesbackground-documents. Accessed April 20, 2016.

${ }^{371}$ EPA. "Environmental Assessment for the Effluent Limitations Guidelines and Standards for the Steam Electric Power Generating Point Source Category." p 2-3. September 2015. https://www.epa.gov/eg/steam-electric-powergenerating-effluent-guidelines-2015-final-rule-documents. Accessed April 20, 2016.

372 Brown, Marilyn A., Daniel D'Arcy, Melissa Lapsa, Isha Sharma, Yufei Li. Solid Waste from the Operation and Decommissioning of Power Plants, Oak Ridge National Laboratory, ORNL/SPR-2016/774 (2016).

${ }^{373}$ Federal Register 40 CFR Part 423. "Effluent Limitations Guidelines and Standards for the Steam Electric Power Generating Point Source Category; Final Rule.” p. 67846-67847. https://www.gpo.gov/fdsys/pkg/FR-2015-1103/pdf/2015-25663.pdf. Accessed April 20, 2016.

${ }^{374}$ EPA. "Technical Development Document for the Effluent Limitations Guidelines and Standards for the Steam Electric Power Generating Point Source Category." p 6-1 through 6-12. EPA-821-R-15-007. September 2015. https://www.epa.gov/eg/steam-electric-power-generating-effluent-guidelines-2015-final-rule-documents. Accessed May 3, 2016.

${ }^{375}$ EPA. "Benefit and Cost Analysis for the Effluent Limitations Guidelines and Standards for the Steam Electric Power Generating Point Source Category." p 1-4. EPA-821-R-15-005. September 2015. https://www.epa.gov/eg/steam-electric-power-generating-effluent-guidelines-2015-final-rule-documents. Accessed April 20, 2016.

${ }^{376}$ EPA. "Benefit and Cost Analysis for the Effluent Limitations Guidelines and Standards for the Steam Electric Power Generating Point Source Category." p 2-1. EPA-821-R-15-005. September 2015. https://www.epa.gov/eg/steam-electric-power-generating-effluent-guidelines-2015-final-rule-documents. Accessed April 20, 2016.

${ }^{377}$ EPA. "Economic, Environmental, and Benefits Analysis of the Final Metal Products and Machinery Rule." p 2016. EPA-821-B-03-002. February 2003. https://www.epa.gov/sites/production/files/2015-11/documents/mpm eeba 2003.pdf. Accessed April 20, 2016.

${ }_{378}$ EPA. "Benefit and Cost Analysis for the Effluent Limitations Guidelines and Standards for the Steam Electric Power Generating Point Source Category." p 2-3. EPA-821-R-15-005. September 2015. https://www.epa.gov/eg/steam-electric-power-generating-effluent-guidelines-2015-final-rule-documents. Accessed April 20, 2016.

${ }^{379}$ EPA. "Benefit and Cost Analysis for the Effluent Limitations Guidelines and Standards for the Steam Electric Power Generating Point Source Category." p 2-4. EPA-821-R-15-005. September 2015.

https://www.epa.gov/eg/steam-electric-power-generating-effluent-guidelines-2015-final-rule-documents. Accessed April 20, 2016.

${ }^{380}$ EPA. "Environmental Assessment for the Effluent Limitations Guidelines and Standards for the Steam Electric Power Generating Point Source Category." Table 3-8 and p 3-1. September 2015. https://www.epa.gov/eg/steamelectric-power-generating-effluent-guidelines-2015-final-rule-documents. Accessed April 20, 2016.

${ }^{381}$ EPA. "Benefit and Cost Analysis for the Effluent Limitations Guidelines and Standards for the Steam Electric Power Generating Point Source Category." p 2-5. EPA-821-R-15-005. September 2015. https://www.epa.gov/eg/steam-electric-power-generating-effluent-guidelines-2015-final-rule-documents. Accessed April 20, 2016.

382 EPA. "Environmental Assessment for the Effluent Limitations Guidelines and Standards for the Steam Electric Power Generating Point Source Category." Table 3-1. September 2015. https://www.epa.gov/eg/steam-electricpower-generating-effluent-guidelines-2015-final-rule-documents. Accessed April 20, 2016.

${ }^{383}$ EPA. "Preventing Eutrophication: Scientific Support for Dual Nutrient Criteria." EPA 820-S-15-001. February 2015. https://www.epa.gov/nutrient-policy-data/preventing-eutrophication-scientific-support-dual-nutrientcriteria. Accessed May 2, 2016. 
${ }^{384}$ EPA. "Environmental Assessment for the Effluent Limitations Guidelines and Standards for the Steam Electric Power Generating Point Source Category." Table 3-2. September 2015. https://www.epa.gov/eg/steam-electricpower-generating-effluent-guidelines-2015-final-rule-documents. Accessed April 20, 2016.

385 EPA. “Technical Support Document for the 2010 Effluent Guidelines Program Plan." Table 5-3. October 2011. EPA-HQ-OW-2009-0819. DCN SE02135.

${ }^{386}$ EPA. “Discharge Monitoring Report (DMR) Pollutant Loading Tool." https://cfpub.epa.gov/dmr/index.cfm. Data accessed on May 2, 2016.

${ }^{387}$ EPA. "Discharge Monitoring Report (DMR) Pollutant Loading Tool."

https://cfpub.epa.gov/dmr/everyday criteria.cfm. Data for the steam electric power generating point source category accessed May 8, 2016.

388 EPA. "Environmental Assessment for the Effluent Limitations Guidelines and Standards for the Steam Electric Power Generating Point Source Category." p 7-5 to 7-6. September 2015. https://www.epa.gov/eg/steam-electricpower-generating-effluent-guidelines-2015-final-rule-documents. Accessed April 29, 2016.

${ }^{389}$ EPA. "Steam Electric Power Generating Point Source Category; Effluent Limitations Guidelines, Pretreatment Standards and New Source Performance Standards." 47 Fed. Reg. 52290. November 1982.https://www.epa.gov/sites/production/files/2015-07/documents/steam-electric final-rule 47-fr52290 111982.pdf. Accessed April 20, 2016.

${ }^{390}$ EPA website. "Industrial Wastewater." https://www.epa.gov/npdes/industrial-wastewater. Accessed April 29, 2016.

${ }^{391}$ Brown, Marilyn A., Daniel D'Arcy, Melissa Lapsa, Isha Sharma, Yufei Li. Solid Waste from the Operation and Decommissioning of Power Plants, Oak Ridge National Laboratory, ORNL/SPR-2016/774 (2016).

392 EPA. "Final Rule: Disposal of Coal Combustion Residuals from Electric Utilities."

https://www.epa.gov/coalash/coal-ash-rule. Accessed April 20, 2016.

${ }^{393}$ EPA. "What is Environmental Justice?" http://www3.epa.gov/environmentaljustice/. Accessed March 3, 2016.

${ }^{394}$ EPA. "Guidance on Considering Environmental Justice During the Development of Regulatory Actions." p. 4.

May 2015. https://www.epa.gov/sites/production/files/2015-06/documents/considering-ej-in-rulemaking-guidefinal.pdf. Accessed June 3, 2016.

${ }^{395}$ EPA. “What is Environmental Justice?" http://www3.epa.gov/environmentaljustice/. Accessed March 3, 2016. ${ }^{396}$ DOE. "Environmental Justice Guidance and Requirements." http://energy.gov/nepa/environmental-justiceguidance-and-requirements. Accessed March 3, 2016.

${ }^{397}$ Gamble, J.L., J. Balbus, M. Berger, K. Bouye, V. Campbell, K. Chief, K. Conlon, A. Crimmins, B. Flanagan, C. Gonzalez-Maddux, E. Hallisey, S. Hutchins, L. Jantarasami, S. Khoury, M. Kiefer, J. Kolling, K. Lynn, A. Manangan, M. McDonald, R. Morello-Frosch, M.H. Redsteer, P. Sheffield, K. Thigpen Tart, J. Watson, K.P. Whyte, and A.F. Wolkin. "The Impacts of Climate Change on Human Health in the United States: A Scientific Assessment." Ch. 9: Populations of Concern. p 249. April 2016. Prepared by the U.S. Global Change Research Program. http://dx.doi.org/10.7930/J0Q81B0T. Accessed May 9, 2016.

${ }^{398}$ Bell, J.E., S.C. Herring, L. Jantarasami, C. Adrianopoli, K. Benedict, K. Conlon, V. Escobar, J. Hess, J. Luvall, C.P. Garcia-Pando, D. Quattrochi, J. Runkle, and C.J. Schreck, III. "The Impacts of Climate Change on Human Health in the United States: A Scientific Assessment." Ch. 4: Impacts of Extreme Events on Human Health. p 104. April 2016. Prepared by the U.S. Global Change Research Program. https://health2016.globalchange.gov/air-quality-impacts. Accessed June 3, 2016.

${ }^{399}$ Fann, N., T. Brennan, P. Dolwick, J.L. Gamble, V. Ilacqua, L. Kolb, C.G. Nolte, T.L. Spero, and L. Ziska. “The Impacts of Climate Change on Human Health in the United States: A Scientific Assessment." Ch. 3: Air Quality Impacts. p 69-98. Prepared by the U.S. Global Change Research Program. https://health2016.globalchange.gov/airquality-impacts. Accessed June 3, 2016.

${ }^{400}$ Gamble, J.L., J. Balbus, M. Berger, K. Bouye, V. Campbell, K. Chief, K. Conlon, A. Crimmins, B. Flanagan, C. Gonzalez-Maddux, E. Hallisey, S. Hutchins, L. Jantarasami, S. Khoury, M. Kiefer, J. Kolling, K. Lynn, A. Manangan, M. McDonald, R. Morello-Frosch, M.H. Redsteer, P. Sheffield, K. Thigpen Tart, J. Watson, K.P. Whyte, and A.F. Wolkin. "The Impacts of Climate Change on Human Health in the United States: A Scientific Assessment." Ch. 9: Populations of Concern. p 249-250. April 2016. Prepared by the U.S. Global Change Research Program. http://dx.doi.org/10.7930/J0Q81B0T. Accessed May 9, 2016. 
${ }^{401}$ Environmental Protection Agency. Air Pollutant Emissions Trends Data, 1970-2014: https://www.epa.gov/airemissions-inventories/air-pollutant-emissions-trends-data. Accessed April 7, 2016.

${ }^{402}$ EPA. "Fact Sheet: Mercury and Air Toxics Standards for Power Plants." p.2. https://www3.epa.gov/mats/pdfs/20111221MATSsummaryfs.pdf. Accessed April 16, 2016.

${ }^{403}$ EPA. "Regulatory Impact Analysis for the Final Mercury and Air Toxics Standards." p 7-35 through 7-42. https://www3.epa.gov/ttn/ecas/regdata/RIAs/matsriafinal.pdf. Accessed May 9, 2016.

${ }^{404}$ EPA. "Regulatory Impact Analysis for the Final Mercury and Air Toxics Standards." p 7-42 through 7-51. https://www3.epa.gov/ttn/ecas/regdata/RIAs/matsriafinal.pdf. Accessed May 9, 2016.

${ }^{405}$ EPA. "Regulatory Impact Analysis for the Final Mercury and Air Toxics Standards." p 7-42 through 7-51. https://www3.epa.gov/ttn/ecas/regdata/RIAs/matsriafinal.pdf. Accessed May 9, 2016.

${ }^{406}$ EPA. "Regulatory Impact Analysis for the Final Mercury and Air Toxics Standards." Table 7-5. https://www.epa.gov/ttn/ecas/regdata/RIAs/matsriafinal.pdf. Accessed May 10, 2016.

${ }^{407}$ Fann, N., T. Brennan, P. Dolwick, J.L. Gamble, V. Ilacqua, L. Kolb, C.G. Nolte, T.L. Spero, and L. Ziska. "The Impacts of Climate Change on Human Health in the United States: A Scientific Assessment." Ch. 3: Air Quality Impacts. p 82. April 2016. Prepared by the U.S. Global Change Research Program. http://dx.doi.org/10.7930/J0GQ6VP6. Accessed June 3, 2016.

${ }^{408}$ Gamble, J.L., J. Balbus, M. Berger, K. Bouye, V. Campbell, K. Chief, K. Conlon, A. Crimmins, B. Flanagan, C. Gonzalez-Maddux, E. Hallisey, S. Hutchins, L. Jantarasami, S. Khoury, M. Kiefer, J. Kolling, K. Lynn, A. Manangan, M. McDonald, R. Morello-Frosch, M.H. Redsteer, P. Sheffield, K. Thigpen Tart, J. Watson, K.P. Whyte, and A.F. Wolkin. "The Impacts of Climate Change on Human Health in the United States: A Scientific Assessment." Ch. 9: Populations of Concern. p 253. April 2016. Prepared by the U.S. Global Change Research Program. http://dx.doi.org/10.7930/J0Q81B0T. Accessed May 9, 2016.

${ }^{409}$ Gamble, J.L., J. Balbus, M. Berger, K. Bouye, V. Campbell, K. Chief, K. Conlon, A. Crimmins, B. Flanagan, C. Gonzalez-Maddux, E. Hallisey, S. Hutchins, L. Jantarasami, S. Khoury, M. Kiefer, J. Kolling, K. Lynn, A. Manangan, M. McDonald, R. Morello-Frosch, M.H. Redsteer, P. Sheffield, K. Thigpen Tart, J. Watson, K.P. Whyte, and A.F. Wolkin. "The Impacts of Climate Change on Human Health in the United States: A Scientific Assessment." Ch. 9: Populations of Concern. p 256-257. April 2016. Prepared by the U.S. Global Change Research Program. http://dx.doi.org/10.7930/J0Q81B0T. Accessed May 9, 2016.

${ }^{410}$ Crocker, D., C. Brown, R. Moolenaar, J. Moorman, C. Bailey, D. Mannino, and F. Holguin. "Racial and ethnic disparities in asthma medication usage and health-care utilization: Data from the National Asthma Survey." October 2009. Chest. vol 136. p 1063-1071. http://dx.doi.org/10.1378/chest.09-0013. Accessed May 6, 2016. ${ }^{411}$ Christian-Smith, J., P. Gleick, H. Cooley, L. Allen, A. Vanderwarker, and K. Berry. "A Twenty-First Century U.S. Water Policy." p 67-68. http://pacinst.org/wp-content/uploads/sites/21/2014/04/21st-centurywater environmental justice ch3.pdf. Accessed May 9, 2016.

${ }^{412}$ EPA. "Benefit and Cost Analysis for the Effluent Limitations Guidelines and Standards for the Steam Electric Power Generating Point Source Category." p 14-1 through 14-7. https://www.epa.gov/sites/production/files/201510/documents/steam-electric benefit-cost-analysis 09-29-2015.pdf. Accessed May 9, 2016.

${ }^{413}$ Xue, J., V. Zartarian, B. Mintz, M. Weber, K. Bailey, and A. Geller. "Modeling tribal exposures to methyl mercury from fish consumption." Science of The Total Environment. Volume 533, November 15, 2015, p 102-109. http://dx.doi.org/10.1016/j.scitotenv.2015.06.070.

${ }^{414}$ EPA. "Benefit and Cost Analysis for the Effluent Limitations Guidelines and Standards for the Steam Electric Power Generating Point Source Category." Table 14-1. https://www.epa.gov/eg/steam-electric-power-generatingeffluent-guidelines-2015-final-rule-documents. Accessed May 9, 2016.

${ }^{415}$ Environmental Protection Agency. "Benefit and Cost Analysis for the Effluent Limitations Guidelines and Standards for the Steam Electric Power Generating Point Source Category." p 14-3. https://www.epa.gov/eg/steam-electric-power-generating-effluent-guidelines-2015-final-rule-documents. Accessed May 9, 2016.

${ }^{416}$ EPA. "Benefit and Cost Analysis for the Effluent Limitations Guidelines and Standards for the Steam Electric Power Generating Point Source Category." p 14-3. https://www.epa.gov/eg/steam-electric-power-generatingeffluent-guidelines-2015-final-rule-documents. Accessed May 9, 2016. 
${ }^{417}$ EPA. "Benefit and Cost Analysis for the Effluent Limitations Guidelines and Standards for the Steam Electric Power Generating Point Source Category." p 14-1 through 14-4. https://www.epa.gov/eg/steam-electric-powergenerating-effluent-guidelines-2015-final-rule-documents. Accessed May 9, 2016.

${ }^{418}$ EPA. "Regulatory Impact Analysis for the Final Mercury and Air Toxics Standards." p 7-41. https://www3.epa.gov/ttn/ecas/regdata/RIAs/matsriafinal.pdf. Accessed May 9, 2016.

${ }^{419}$ Cunliff C. and C. Murphy. "Environment Baseline Volume 1: Greenhouse Gas Emissions from the U.S. Power Sector." Office of Energy Policy and Systems Analysts, U.S. Department of Energy, May 2016.

${ }^{420}$ EPA. "Inventory of U.S. Greenhouse Gas Emissions and Sinks: 1990-2013." p.1-3. April 2015. http://www3.epa.gov/climatechange/ghgemissions/usinventoryreport.html. Accessed February 4, 2016. ${ }^{421}$ NOAA. "Vital Signs of the Planet." http://climate.nasa.gov/causes/. Accessed February 4, 2016.

${ }^{422}$ Balbus, J., A. Crimmins, J.L. Gamble, D.R. Easterling, K.E. Kunkel, S. Saha, and M.C. Sarofim. "Climate Change and Human Health. The Impacts of Climate Change on Human Health in the United States: A Scientific Assessment." $p$ 25-42. April 2016. U.S. Global Change Research Program, Washington, DC. http://dx.doi.org/10.7930/JOVXODFW. Accessed May 9, 2016.

423 IPCC, 2014: Summary for policymakers. In: "Climate Change 2014: Impacts, Adaptation, and Vulnerability. Part A: Global and Sectoral Aspects." Contribution of Working Group II to the Fifth Assessment Report of the Intergovernmental Panel on Climate Change [Field, C.B., V.R. Barros, D.J. Dokken, K.J. Mach, M.D. Mastrandrea, T.E. Bilir, M. Chatterjee, K.L. Ebi, Y.O. Estrada, R.C. Genova, B. Girma, E.S. Kissel, A.N. Levy, S. MacCracken, P.R. Mastrandrea, and L.L. White (eds.)]. Cambridge University Press, Cambridge, United Kingdom and New York, NY, USA, p. 6. http://www.ipcc-wg2.gov/AR5/. Accessed May 9, 2016.

${ }^{424}$ EPA. "Endangerment and Cause or Contribute Findings for Greenhouse Gases Under Section 202(a) of the Clean Air Act." 74 Fed. Reg. 66,496 (Dec. 15, 2009). https://www3.epa.gov/climatechange/endangerment/. Accessed May 9, 2016.

425 EPA. "Regulatory Impact Analysis for the Clean Power Plan Final Rule." p 7-18 through 7-21. https://www.epa.gov/sites/production/files/2015-08/documents/cpp-final-rule-ria.pdf. Accessed May 9, 2016 ${ }^{426}$ Bell, J.E., S.C. Herring, L. Jantarasami, C. Adrianopoli, K. Benedict, K. Conlon, V. Escobar, J. Hess, J. Luvall, C.P. Garcia-Pando, D. Quattrochi, J. Runkle, and C.J. Schreck, III. "The Impacts of Climate Change on Human Health in the United States: A Scientific Assessment." Ch. 4: Impacts of Extreme Events on Human Health. p 100. April 2016. Prepared by the U.S. Global Change Research Program. https://health2016.globalchange.gov/extreme-events. Accessed June 3, 2016.

${ }^{427}$ Gamble, J.L., J. Balbus, M. Berger, K. Bouye, V. Campbell, K. Chief, K. Conlon, A. Crimmins, B. Flanagan, C. Gonzalez-Maddux, E. Hallisey, S. Hutchins, L. Jantarasami, S. Khoury, M. Kiefer, J. Kolling, K. Lynn, A. Manangan, M. McDonald, R. Morello-Frosch, M.H. Redsteer, P. Sheffield, K. Thigpen Tart, J. Watson, K.P. Whyte, and A.F. Wolkin. "The Impacts of Climate Change on Human Health in the United States: A Scientific Assessment." Ch. 9: Populations of Concern. p 256-257. April 2016. Prepared by the U.S. Global Change Research Program. http://dx.doi.org/10.7930/J0Q81B0T. Accessed May 9, 2016.

${ }^{428}$ Bell, J.E., S.C. Herring, L. Jantarasami, C. Adrianopoli, K. Benedict, K. Conlon, V. Escobar, J. Hess, J. Luvall, C.P. Garcia-Pando, D. Quattrochi, J. Runkle, and C.J. Schreck, III. "The Impacts of Climate Change on Human Health in the United States: A Scientific Assessment." Ch. 4: Impacts of Extreme Events on Human Health. p 99-128. April 2016. Prepared by the U.S. Global Change Research Program. http://dx.doi.org/10.7930/JOBZ63ZVGCRP. Accessed May 9, 2016.

${ }^{429}$ Executive Order No. 12898. "Federal Actions to Address Environmental Justice in Minority Populations and LowIncome Populations." 59 Fed. Reg. 7629. February 11, 1994. http://www.archives.gov/federal-register/executiveorders/pdf/12898.pdf. Accessed March 3, 2016.

${ }^{430}$ The White House. "Presidential Proclamation - 20th Anniversary of Executive Order 12898 on Environmental Justice." February 10, 2014. https://www.whitehouse.gov/the-press-office/2014/02/10/presidential-proclamation20th-anniversary-executive-order-12898-environ. Accessed March 3, 2016.

${ }^{431}$ Council on Environmental Quality. "Environmental Justice: Guidance Under the National Environmental Policy Act." 1997. https://ceq.doe.gov/nepa information/justice.html. Accessed June 3, 2016.

432 EPA. "Draft EJ 2020 Action Agenda Framework." p 1. https://www.epa.gov/environmentaljustice/draftenvironmental-justice-2020-action-agenda-framework. Accessed May 9, 2016. 
${ }^{433}$ EPA. "Guidance on Considering Environmental Justice During the Development of Regulatory Actions." https://www.epa.gov/sites/production/files/2015-06/documents/considering-ej-in-rulemaking-guide-final.pdf. Accessed May 9, 2016.

${ }^{434}$ EPA. "Ejscreen." https://www.epa.gov/ejscreen. Accessed May 9, 2016.

${ }^{435}$ EPA. "Plan EJ 2014: Legal Tools." https://www.epa.gov/sites/production/files/201504/documents/planej2014legaltools.pdf. Accessed May 9, 2016.

${ }^{436}$ EPA. "Cover Memo for Guidance on Considering Environmental Justice in the Development of a Regulatory Action." https://www.epa.gov/environmentaljustice/guidance-considering-environmental-justice-duringdevelopment-action. Accessed May 9, 2016.

${ }^{437}$ EPA. "Technical Documentation for EJSCREEN." p 1-8. https://www.epa.gov/sites/production/files/201505/documents/ejscreen technical document 20150505.pdf. Accessed May 9, 2016.

${ }^{438}$ Tonn, B., D. Carroll, E. Rose, B. Hawkins, S. Pigg, D. Bausch, G. Dalhoff, M. Blasnik, J. Eisenberg, C. Cowan, B. Conlon. "Weatherization Works II - Summary of Findings from the ARRA Period Evaluation of the U.S. Department of Energy's Weatherization Assistance Program." P xvi-xvii. July 2015.

http://weatherization.ornl.gov/RecoveryActpdfs/ORNL TM-2015 139.pdf. Accessed June 1, 2016.

${ }^{439}$ DOE. "A View of Environmental Justice at the U.S. Department of Energy." p 10. February 11, 2014. http://energy.gov/nepa/downloads/view-environmental-justice-us-department-energy-doe-2014. Accessed May 9, 2016.

${ }^{440}$ DOE. "Environmental Justice: Five-Year Implementation Plan Third and Fourth Annual Progress Reports." p 2750. December 24, 2013. http://energy.gov//m/downloads/environmental-justice-five-year-implementation-planthird-and-fourth-annual-progress. Accessed May 9, 2016.

${ }^{441}$ Environmental Protection Agency. Air Pollutant Emissions Trends Data, 1970-2014: https://www.epa.gov/airemissions-inventories/air-pollutant-emissions-trends-data. Accessed April 7, 2016.

${ }^{442}$ Environmental Protection Agency. Air Pollutant Emissions Trends Data, 1970-2014: https://www.epa.gov/airemissions-inventories/air-pollutant-emissions-trends-data. Accessed April 7, 2016.

${ }^{443}$ Energy Information Administration. Monthly Energy Review. 2016: Table 7.2b Electricity Net Generation: Electric Power Sector: http://www.eia.gov/totalenergy/data/monthly/. Accessed April 7, 2016.

${ }^{444}$ Environmental Protection Agency. Air Pollutant Emissions Trends Data, 1970-2014."

${ }^{445}$ Energy Information Administration. Monthly Energy Review. 2016: Table 7.2b Electricity Net Generation: Electric Power Sector.

${ }^{446}$ EIA. "Annual Electric Generator Report." https://www.eia.gov/electricity/data/eia860. Accessed May 10, 2016.

${ }^{447}$ EIA. "Monthly Energy Review." http://www.eia.gov/totalenergy/data/monthly. Accessed May 10, 2016.

${ }^{448}$ EIA. "Form EIA-923 Detailed Data." https://www.eia.gov/electricity/data/eia923. Accessed May 10, 2016.

${ }^{449}$ EPA. "National Emissions Inventory." http://www.epa.gov/air-emissions-inventories/2011-national-emissionsinventory-nei-data. Accessed May 10, 2016.

${ }^{450}$ National Research Council (NRC). Committee on Health, Environmental, Other External Costs, Benefits of Energy Production, and Consumption. 2010. Hidden costs of energy: unpriced consequences of energy production and use. National Academies Press (2010). Page 92.

http://www.nap.edu/login.php?record id=12794\&page=http\%3A\%2F\%2Fwww.nap.edu\%2Fdownload.php\%3Frec ord id\%3D12794

${ }^{451}$ Muller, Nicholas Z., Robert Mendelsohn, and William Nordhaus. "Environmental accounting for pollution in the United States economy." The American Economic Review (2011): 1649-1675. Table 5, p. 1670.

http://www.jstor.org/stable/23045618

${ }_{452}$ Muller, Nicholas Z. and Robert Mendelsohn. "Measuring the damages of air pollution in the United States." Journal of Environmental Economics and Management 54, no. 1 (2007): 1-14.

http://dx.doi.org/10.1016/i.jeem.2006.12.002.

453 Jaramillo, Paulina and Nicholas Z. Muller. "Air pollution emissions and damages from energy production in the US: 2002-2011." Energy Policy 90 (2016): 202-211. Table 1, page 206.

http://www.sciencedirect.com/science/article/pii/S0301421515302494

${ }^{454}$ Burtraw, Dallas et al. 1998. "Costs and benefits of reducing air pollutants related to acid rain." Contemporary Economic Policy 16, no. 4 (1998): 379-400. 
455 Environmental Protection Agency. "Benefits and Costs of the Clean Air Act 1990-2020, the Second Prospective Study." (Washington, DC: Environmental Protection Agency, Office of Air and Radiation, 2011.

${ }^{456}$ National Research Council (NRC). Committee on Health, Environmental, Other External Costs, Benefits of Energy Production, and Consumption. 2010. Hidden costs of energy: unpriced consequences of energy production and use. National Academies Press (2010).

${ }^{457}$ Muller, Nicholas Z. and Robert Mendelsohn. "Efficient pollution regulation: getting the prices right." The American Economic Review (2009): 1714-1739. Page 1734. http://www.jstor.org/stable/25592534

458 Muller, Nicholas Z., Robert Mendelsohn, and William Nordhaus. "Environmental accounting for pollution in the United States economy." The American Economic Review (2011): 1649-1675.

http://www.jstor.org/stable/23045618

${ }^{459}$ National Research Council, Committee on Health, Environmental, Other External Costs, Benefits of Energy Production, and Consumption. 2010. Page 92.

${ }^{460}$ Muller, Nicholas Z., Robert Mendelsohn, and William Nordhaus. "Environmental accounting for pollution in the United States economy." The American Economic Review (2011): 1649-1675. Table 5, p. 1670.

http://www.jstor.org/stable/23045618

${ }^{461}$ Muller, Nicholas Z. and Robert Mendelsohn. "Measuring the damages of air pollution in the United States." Journal of Environmental Economics and Management 54, no. 1 (2007): 1-14. http://dx.doi.org/10.1016/i.jeem.2006.12.002.

462 Jaramillo, Paulina and Nicholas Z. Muller. "Air pollution emissions and damages from energy production in the US: 2002-2011." Energy Policy 90 (2016): 202-211. Table 1, page 206.

463 NAPAP. National Acid Precipitation Assessment Program Report to Congress: An Integrated Assessment.

(Washington, D.C.: National Science and Technology Council, Committee on Environmental and Natural Resources, 2005: 8, 12). http://www.esrl.noaa.gov/csd/AQRS/reports/napapreport05.pdf

${ }^{464}$ Environmental Protection Agency. Air Pollutant Emissions Trends Data, 1970-2014: https://www.epa.gov/airemissions-inventories/air-pollutant-emissions-trends-data. Accessed April 7, 2016.

465 Burtraw, Dallas, Alan Krupnick, Erin Mansur, Daivd Austin, and Deirdre Farrell. "Costs and benefits of reducing air pollutants related to acid rain." Contemporary Economic Policy 16, no. 4 (1998): 379. Figure 1, page 388. http://onlinelibrary.wiley.com/doi/10.1111/i.1465-7287.1998.tb00527.x/abstract

${ }^{466}$ Carlson, Curtis, Dallas Burtraw, Maureen Cropper, and Karen L. Palmer. 2000. "Sulfur Dioxide Control by Electric Utilities: What Are the Gains from Trade?" Journal of Political Economy 108, no. 6 (2000): 1313 Table 2.

doi:10.1086/317681.

${ }^{467}$ Chan, G. et al. The $\mathrm{SO}_{2}$ Allowance Trading System and the Clean Air Act Amendments of 1990: Reflections on Twenty Years of Policy Innovation.

${ }^{468}$ Muller, N.Z. and R.O. Mendelsohn. "Measuring the damages of air pollution in the United States." Journal of Environmental Economics and Management 54, no. 1 (2007): 8.

${ }^{469}$ Chan, G. et al. The $\mathrm{SO}_{2}$ Allowance Trading System and the Clean Air Act Amendments of 1990: Reflections on Twenty Years of Policy Innovation. (Cambridge, MA: Harvard Kennedy School, 2012).

${ }^{470}$ Department of Energy. "Quadrennial Energy Review: Energy Transmission, Storage and Distribution Infrastructure." p 7-3 - 7-6. April 2016. http://www.energy.gov/epsa/quadrennial-energy-review-first-installment

${ }^{471}$ White House, 2015. "Modernizing our Electric transmission Infrastructure and Driving the Development of Clean Energy." July, 2015 Report. https://www.whitehouse.gov/blog/2015/07/21/modernizing-our-electrictransmission-infrastructure-and-driving-development-clean-e

472 EURELECTRIC, 2011. "Life Cycle Assessment of Electricity Generation." WG Environmental Management \& Economics. Page. 14. http://www.eurelectric.org/media/26740/report-Ica-resap-final-2011-420-0001-01-e.pdf.

${ }^{473}$ Fthenakis, V., and H.-Y. Kim. "Land Use and Electricity Generation: A Life -cycle Analysis." Renewable and Sustainable Energy Reviews 13, no. 6-7 (2009): 1465-1474.

http://www.sciencedirect.com/science/article/pii/S1364032108001354. Accessed May 2016.

${ }^{474}$ Sovacool, B.K. 2008. Valuing the greenhouse gas emissions from nuclear power: A critical survey. Energy Policy 36(8):2950-2963. Available at:

http://www.biol.uw.edu.pl/pl/files/docs/st_dokt/SD_SCB Bird_and_bat_mortalities.pdf. 
${ }^{475}$ Conniff, Richard. "Electric Power Rights of Way: A New Frontier for Conservation." Yale Environment 360. 2014. http://e360.yale.edu/feature/electric power rights of way a new frontier for conservation/2816/

${ }^{476}$ EPA 821-R-09-008. "Steam Electric Power Generating Point Source Category: Final Detailed Study Report." p xii. October 2009. https://www.epa.gov/eg/steam-electric-power-generating-effluent-guidelines-backgrounddocuments. Accessed April 20, 2016.

477 EPA. "Steam Electric Effluent Guidelines - 2015 Final Rule; Fact Sheet." https://www.epa.gov/sites/production/files/2015-10/documents/steam-electric-final-rule-factsheet 10-012015.pdf. Accessed April 11, 2016.

${ }^{478}$ EPA. "Economic, Environmental, and Benefits Analysis of the Final Metal Products and Machinery Rule." p 2016. EPA-821-B-03-002. February 2003. https://www.epa.gov/sites/production/files/2015-11/documents/mp$m$ eeba 2003.pdf. Accessed April 20, 2016.

${ }^{479}$ EPA. "Benefit and Cost Analysis for the Effluent Limitations Guidelines and Standards for the Steam Electric Power Generating Point Source Category." p 2-1. EPA-821-R-15-005. September 2015.

https://www.epa.gov/eg/steam-electric-power-generating-effluent-guidelines-2015-final-rule-documents. Accessed April 20, 2016.

480 EPA. "Benefit and Cost Analysis for the Effluent Limitations Guidelines and Standards for the Steam Electric Power Generating Point Source Category." p 2-3. EPA-821-R-15-005. September 2015. https://www.epa.gov/eg/steam-electric-power-generating-effluent-guidelines-2015-final-rule-documents. Accessed April 20, 2016.

${ }^{481}$ EPA. "Environmental Assessment for the Effluent Limitations Guidelines and Standards for the Steam Electric Power Generating Point Source Category." Table 3-8 and p 3-1. September 2015. https://www.epa.gov/eg/steamelectric-power-generating-effluent-guidelines-2015-final-rule-documents. Accessed April 20, 2016.

${ }^{482}$ EPA. "Environmental Assessment for the Effluent Limitations Guidelines and Standards for the Steam Electric Power Generating Point Source Category." p 7-5 to 7-6. September 2015. https://www.epa.gov/eg/steam-electricpower-generating-effluent-guidelines-2015-final-rule-documents. Accessed April 29, 2016.

${ }^{483}$ EPA. "Final Rule: Disposal of Coal Combustion Residuals from Electric Utilities." https://www.epa.gov/coalash/coal-ash-rule. Accessed April 20, 2016.

${ }^{484}$ EPA. “What is Environmental Justice?" http://www3.epa.gov/environmentaljustice/. Accessed March 3, 2016.

${ }^{485}$ EPA. "What is Environmental Justice?" http://www3.epa.gov/environmentaljustice/. Accessed March 3, 2016.

${ }^{486}$ DOE. "Environmental Justice Guidance and Requirements." http://energy.gov/nepa/environmental-justiceguidance-and-requirements. Accessed March 3, 2016.

${ }^{487}$ Epa mats ria. Section 7.11.1.2. EPA. "Regulatory Impact Analysis for the Final Mercury and Air Toxics Standards." Section 7.11.1.2. https://www3.epa.gov/ttnecas1/regdata/RIAs/matsriafinal.pdf. Accessed May 19, 2016.

${ }^{488}$ Executive Order No. 12898. "Federal Actions to Address Environmental Justice in Minority Populations and LowIncome Populations." 59 Fed. Reg. 7629. February 11, 1994. http://www.archives.gov/federal-register/executiveorders/pdf/12898.pdf. Accessed March 3, 2016.

${ }^{489}$ The White House. "Presidential Proclamation - 20th Anniversary of Executive Order 12898 on Environmental Justice." February 10, 2014. https://www.whitehouse.gov/the-press-office/2014/02/10/presidential-proclamation20th-anniversary-executive-order-12898-environ. Accessed March 3, 2016.

${ }^{490}$ Council on Environmental Quality. "Environmental Justice: Guidance Under the National Environmental Policy Act." 1997. http://www3.epa.gov/environmentaljustice/resources/policy/ej guidance nepa ceq1297.pdf. Accessed March 3, 2016.

${ }^{491}$ Environment Protection Agency, National Emissions Inventory (NEI). 2015. https://www.epa.gov/air-emissionsinventories/air-pollutant-emissions-trends-data, Accessed January 142016.

${ }^{492}$ Energy Information Administration, Annual Energy Review. 2012: Table 2.1F. http://www.eia.gov/totalenergy/data/annual/, Accessed January 142016

${ }^{493}$ Kaya, Y. "Impact of carbon dioxide emission control on GNP growth: Interpretation of proposed scenarios." Paper presented at the IPCC Energy and Industry Subgroup, Response Strategies Working Group, Paris, France. 1990. 
${ }^{494}$ Intergovernmental Panel for Climate Change. "Summary for policymakers: a report of Working Group I of the Intergovernmental Panel on Climate Change." Climate change, 1-20. 2001: Section 3.1.

http://www.ipcc.ch/ipccreports/sres/emission/index.php?idp=0, Accessed in March 14, 2016.

${ }_{495}$ Goodarzi, F. (2006). "Assessment of elemental content of milled coal, combustion residues, and stack emitted materials: possible environmental effects for a Canadian pulverized coal-fired power plant." International Journal of Coal Geology. 65 no. 1 (2006): 17-25.

${ }^{496}$ Sheng, C. et al. Comparison of sulphur retention by coal ash in different types of combustors." Fuel Processing Technology. 64 no. 1 (2000): 1-11.

${ }^{497}$ Energy Information Administration, Form EIA-923 detailed data, 1995-2015, https://www.eia.gov/electricity/data/eia923/, Accessed January 142016.

${ }^{498}$ Energy Information Administration Cost and Quality of Fuels for Electric Plants Report, 1995-2010, https://www.eia.gov/electricity/cost quality/. Accessed January 142016

${ }^{499}$ Energy Information Administration, Form EIA-923 detailed data, 2015, https://www.eia.gov/electricity/data/eia923/, Table A5, Accessed January 142016.

${ }^{500}$ Tribal Energy and Environmental Information Clearinghouse (TEEIC), http://teeic.indianaffairs.gov/images/photos/CoalBearingAreas USGS.gif. Accessed January 14th 2016

${ }^{501}$ Energy Information Administration, Coal Industry Annual, April 2015, Table 6, http://www.eia.gov/coal/annual/pdf/acr.pdf. Accessed January $14^{\text {th }} 2016$

${ }^{502}$ Energy Information Administration Cost and Quality of Fuels for Electric Plants Report, 1995-2010, https://www.eia.gov/electricity/cost quality/. Accessed January $14^{\text {th }} 2016$

${ }^{503}$ Energy Information Administration, Form EIA-923 detailed data, 2015, https://www.eia.gov/electricity/data/eia923/, Table A5, Accessed January 14 2016.

${ }^{504}$ Energy Information Administration, Coal Industry Annual, April 2015, Table 31, http://www.eia.gov/coal/annual/pdf/acr.pdf. Accessed January $14^{\text {th }} 2016$

${ }^{505}$ Energy Information Administration, Monthly Energy Review, 1994-2015, Table 8.4b, http://www.eia.gov/totalenergy/data/annual/, Accessed January 14 2016)

${ }^{506}$ Energy Information Administration, Monthly Energy Review, 1995-2015, Table 8.2c, http://www.eia.gov/totalenergy/data/annual/, Accessed January 142016

${ }^{507}$ Energy Information Administration, Monthly Energy Review, 1995-2015, Table 7.2, http://www.eia.gov/totalenergy/data/annual/. Accessed January $14^{\text {th }} 2016$

${ }^{508}$ Energy Information Administration, Electric Power Annual, February 2016, Table 9.2, http://www.eia.gov/electricity/annual/. Accessed March 14 2016.

${ }^{509}$ Energy Information Administration, Electric Power Annual, February 2016, Table 9.2, http://www.eia.gov/electricity/annual/. Accessed March 14 2016.

${ }^{510}$ Oak Ridge National Laboratory and Resources for the Future (RFF). External Costs and Benefits of Fuel Cycles (Reports 2-8). McGraw-Hill Utility Data Institute, Washington, 1994-1998. 1995.

${ }^{511}$ European Commission. Externalities of Energy: Volume 1: Summary; Volume 2: Methodology; Volume 3: Coal and Lignite; Volume 4: Oil and Gas; Volume 5: Nuclear; Volume 6: Wind and Hydro Fuel Cycles. European Commission, Directorate-General XII. Science, Research and Development. 1995.

512 Oak Ridge National Laboratory and Resources for the Future. "Estimating Externalities of Nuclear Fuel Cycles." Report 8. 1995: iv. http://web.ornl.gov/info/reports/1995/3445604259148.pdf.

513 Industrial Economics. "Health and Welfare Benefits Analyses to Support the Second Section 812 Benefit-Cost Analysis of the Clean Air Act."

${ }^{514}$ Environmental Protection Agency. Benefits and Costs of the Clean Air Act 1990-2020, the Second Prospective Study. (Washington, DC: Environmental Protection Agency, Office of Air and Radiation, 2011).

${ }^{515}$ Muller, Mendelsohn, and Nordhaus 2011.

516 Muller and Mendelsohn. 2007.

${ }^{517}$ National Research Council. Environmental Impacts of Wind-Energy Projects. (Washington, DC: National Academies Press, 2007.)

518 Parry, I.W.H. et al. Getting Energy Prices Right: From Principle to Practice. (International Monetary Fund, 2014).

519 Industrial Economics 2011. 
${ }^{520}$ Environmental Protection Agency. "Benefits and Costs of the Clean Air Act 1990-2020, the Second Prospective Study." (Washington, DC: Environmental Protection Agency, Office of Air and Radiation, 2011).

${ }^{521}$ National Research Council, Committee on Health, Environmental, Other External Costs, Benefits of Energy Production, and Consumption. Hidden costs of energy: unpriced consequences of energy production and use. (National Academies Press, 2010). DOI: 10.17226/12794

522 Muller and Mendelsohn 2007.

${ }^{523}$ National Research Council, Committee on Health, Environmental, Other External Costs, Benefits of Energy Production, and Consumption 2010.

${ }^{524}$ National Research Council, Committee on Health, Environmental, Other External Costs, Benefits of Energy Production, and Consumption 2010.

${ }^{525}$ Epstein, Paul R. et al. 2011. "Full cost accounting for the life cycle of coal." Annals of the New York Academy of Sciences 1219, no. 1 (2011): 73-98.

${ }^{526}$ Pope III, C Arden et al. "Lung cancer, cardiopulmonary mortality, and long-term exposure to fine particulate air pollution." JAMA 287, no. 9 (2002): 1132-1141.

${ }^{527}$ Roman et al. Expert judgment assessment of the mortality impact of changes in ambient fine particulate matter in the US."Environmental science \& technology 42 (7):2268-2274.

${ }^{528}$ Schwartz et al. "The effect of dose and timing of dose on the association between airborne particles and survival." Environmental Health Perspectives. 2008: 64-69.

${ }^{529}$ Pope III, C. Arden et al. "Lung cancer, cardiopulmonary mortality, and long-term exposure to fine particulate air pollution." JAMA 287, no. 9 (2002): 1132-1141.

${ }^{530}$ Burtraw, Dallas et al. 1998. "Costs and benefits of reducing air pollutants related to acid rain." Contemporary Economic Policy 16, no. 4 (1998): 379-400.

${ }^{531}$ Environmental Protection Agency. "Benefits and Costs of the Clean Air Act 1990-2020, the Second Prospective Study." (Washington, DC: Environmental Protection Agency, Office of Air and Radiation, 2011.

${ }^{532}$ National Research Council (NRC). Committee on Health, Environmental, Other External Costs, Benefits of Energy Production, and Consumption. 2010. Hidden costs of energy: unpriced consequences of energy production and use. National Academies Press (2010).

${ }^{533}$ Muller, Nicholas Z. and Robert Mendelsohn. "Efficient pollution regulation: getting the prices right." The American Economic Review (2009): 1714-1739. Page 1734. http://www.jstor.org/stable/25592534.

534 Muller, Nicholas Z., Robert Mendelsohn, and William Nordhaus. "Environmental accounting for pollution in the United States economy." The American Economic Review (2011): 1649-1675.

http://www.jstor.org/stable/23045618.

${ }^{535}$ Viscusi, W Kip and Joseph E Aldy. "The value of a statistical life: a critical review of market estimates throughout the world." Journal of risk and uncertainty 27, no. 1 (2003): 5-76.

${ }^{536}$ Viscusi, W Kip and Joseph E Aldy. "The value of a statistical life: a critical review of market estimates throughout the world." 2003.

${ }^{537}$ Environmental Protection Agency. Valuing the Protection of Ecological Systems and Services. 2009.

${ }^{538}$ EPA. "Distributed Generation." http://www2.epa.gov/energy/distributed-generation. Accessed April 20, 2016.

${ }^{539}$ EIA. "Electric Power Monthly." Tables 1.2.C, 1.2.D and 1.2.E. Release Date February 26, 2016. http://www.eia.gov/electricity/monthly/. Accessed March 18, 2016.

${ }^{540}$ EIA. "EIA electricity data now include estimated small-scale solar PV capacity and generation." December 2, 2015. http://www.eia.gov/todayinenergy/detail.cfm?id=23972. Accessed April 20, 2016.

${ }^{541}$ EIA. "Electricity Data Browser." http://www.eia.gov/electricity/data/browser/. Accessed April 4, 2016.

${ }^{542}$ EIA. "Electricity Data Browser." http://www.eia.gov/electricity/data/browser/. Accessed April 4, 2016.

${ }^{543}$ EIA. "Detailed State Data." U.S. Electric Power Industry Estimated Emissions by State. October 21, 2015. http://www.eia.gov/electricity/data/state. Accessed March 18, 2016.

${ }^{544}$ Sathaye, J., O. Lucon, A. Rahman, J. Christensen, F. Denton, J. Fujino, G. Heath, S. Kadner, M. Mirza, H. Rudnick, A. Schlaepfer, A. Shmakin, 2011: Renewable Energy in the Context of Sustainable Development. In IPCC Special Report on Renewable Energy Sources and Climate Change Mitigation [O. Edenhofer, R. Pichs-Madruga, Y. Sokona, K. Seyboth, P. Matschoss, S. Kadner, T. Zwickel, P. Eickemeier, G. Hansen, S. Schlömer, C. von Stechow (eds)], 
Cambridge University Press, Cambridge, United Kingdom and New York, NY, USA. p. 730. http://srren.ipccwg3.de/report/IPCC SRREN_Ch09.pdf. Accessed April 12, 2016.

${ }^{545}$ Eric Masanet, Yuan Chang, Anand R. Gopal, Peter Larsen, William R. Morrow III, Roger Sathre, Arman Shehabi, and Pei Zhai. "Life-Cycle Assessment of Electric Power Systems." p 110-111. Annual Review of Environment and Resources, Vol. 38: 107 -136 (Volume publication date October 2013). Accessed April 12, 2016.

${ }^{546}$ Sathaye, J., O. Lucon, A. Rahman, J. Christensen, F. Denton, J. Fujino, G. Heath, S. Kadner, M. Mirza, H. Rudnick, A. Schlaepfer, A. Shmakin, 2011: Renewable Energy in the Context of Sustainable Development. In IPCC Special Report on Renewable Energy Sources and Climate Change Mitigation [O. Edenhofer, R. Pichs-Madruga, Y. Sokona, K. Seyboth, P. Matschoss, S. Kadner, T. Zwickel, P. Eickemeier, G. Hansen, S. Schlömer, C. von Stechow (eds)], Cambridge University Press, Cambridge, United Kingdom and New York, NY, USA. p. 730. http://srren.ipccwg3.de/report/IPCC SRREN Ch09.pdf. Accessed April 12, 2016.

${ }^{547}$ International Organization for Standardization, EN ISO 14040. "Environmental management-Life cycle assessment-Principles and framework." 2006.

${ }^{548}$ European Commission-Joint Research Centre-Institute for Environment and Sustainability, EC-JRC. "International Reference Life Cycle Data System (ILCD) Handbook." Luxembourg, Publications Office of the European Union; 2012. http://eplca.jrc.ec.europa.eu/uploads/JRC-Reference-Report-ILCD-Handbook-Towardsmore-sustainable-production-and-consumption-for-a-resource-efficient-Europe.pdf.

${ }^{549}$ World Health Organization. "Air Quality Guidelines. Global Update 2005. Particulate Matter, Ozone, Nitrogen Dioxide and Sulfur Dioxide." http://www.euro.who.int/ data/assets/pdf file/0005/78638/E90038.pdf. Accessed April 21, 2016.

${ }^{550}$ Sathaye, J., O. Lucon, A. Rahman, J. Christensen, F. Denton, J. Fujino, G. Heath, S. Kadner, M. Mirza, H. Rudnick, A. Schlaepfer, A. Shmakin, 2011: Renewable Energy in the Context of Sustainable Development. In IPCC Special Report on Renewable Energy Sources and Climate Change Mitigation [O. Edenhofer, R. Pichs-Madruga, Y. Sokona, K. Seyboth, P. Matschoss, S. Kadner, T. Zwickel, P. Eickemeier, G. Hansen, S. Schlömer, C. von Stechow (eds)], Cambridge University Press, Cambridge, United Kingdom and New York, NY, USA. p. 736-739. http://srren.ipccwg3.de/report/IPCC SRREN Ch09.pdf. Accessed April 12, 2016.

${ }^{551}$ Sathaye, J., O. Lucon, A. Rahman, J. Christensen, F. Denton, J. Fujino, G. Heath, S. Kadner, M. Mirza, H. Rudnick, A. Schlaepfer, A. Shmakin, 2011: Renewable Energy in the Context of Sustainable Development. In IPCC Special Report on Renewable Energy Sources and Climate Change Mitigation [O. Edenhofer, R. Pichs-Madruga, Y. Sokona, K. Seyboth, P. Matschoss, S. Kadner, T. Zwickel, P. Eickemeier, G. Hansen, S. Schlömer, C. von Stechow (eds)], Cambridge University Press, Cambridge, United Kingdom and New York, NY, USA. p. 738. http://srren.ipccwg3.de/report/IPCC SRREN Ch09.pdf. Accessed April 12, 2016. 